Function and dynamics of aptamers: A case study on the malachite green aptamer

by

\title{
Tianjiao Wang
}

A dissertation submitted to the graduate faculty

in partial fulfillment of the requirements for the degree of

DOCTOR OF PHILOSOPHY

Major: Molecular, Cellular and Developmental Biology
Program of Study Committee:
Marit Nilsen-Hamilton, Major Professor
Drena Dobbs
Monica Lamm
Gustavo MacIntosh
Allen Miller

Iowa State University

Ames, Iowa

2008

Copyright (C Tianjiao Wang, 2008. All rights reserved. 


\section{TABLE OF CONTENTS}

ABSTRACT

CHAPTER 1. GENERAL INTRODUCTION 1

DISSERTATION ORGANIZATION 1

RESEARCH AIMS AND SIGNIFICANCE 3

Aim 1: Controlling chemical reactivity by an aptamer 3

Aim 2: Developing MD simulation as a tool to study aptamer-target interactions

with experimental validation.

REFERENCES

CHAPTER 2. LITERATURE REVIEW $\quad 6$

$\begin{array}{ll}\text { APTAMER } & 6\end{array}$

$\begin{array}{ll}\text { APTAMER MODIFICATIONS } & 7\end{array}$

$\begin{array}{ll}\text { MULTIVALENT APTAMERS } & 17\end{array}$

$\begin{array}{ll}\text { SELEX } & 20\end{array}$

TABLES AND FIGURES 31

REFERENCES $\quad 51$

CHAPTER 3. STERIC PROTECTION OF MOLECULAR REACTIVITY BY AN APTAMER

$\begin{array}{ll}\text { ABSTRACT } & 74\end{array}$

$\begin{array}{ll}\text { INTRODUCTION } & 74\end{array}$

EXPERIMENTAL PROCEDURES 76

$\begin{array}{ll}\text { RESULTS } & 80\end{array}$

DISCUSSION 83

REFERENCES 86

$\begin{array}{ll}\text { FOOTNOTES } & 88\end{array}$

FIGURE LEGENDS

$\begin{array}{ll}\text { TABLES } & 92\end{array}$

$\begin{array}{ll}\text { FIGURES } & 93\end{array}$ 
CHAPTER 4. TWO-STEP MECHANISM OF MALACHITE GREEN-MALACHITE GREEN APTAMER RECOGNITION

$\begin{array}{ll}\text { ABSTRACT } & 101\end{array}$

INTRODUCTION 102

$\begin{array}{ll}\text { RESULTS } & 104\end{array}$

DISCUSSION 110

MATERIALS AND METHODS 114

ACKNOWLDEGEMENT 119

REFERENCES 119

FIGURE LEGENDS 124

TABLES 127

$\begin{array}{ll}\text { FIGURES } & 130\end{array}$

CHAPTER 5. GENERAL CONCLUSIONS 138

CONTROLLING CHEMICAL REACTIVITY WITH APTAMERS 138

DYNAMICS OF AN APTAMER-TARGET INTERACTION REVEALED BY MD SIMULATION WITH EXPERIMENTAL VALIDATION 139

PERSPECTIVE 140

$\begin{array}{ll}\text { REFERENCES } & 141\end{array}$

APPENDIX. SELECTING APTAMERS AGAINST MOUSE LCN2 PROETIN 142

ABSTRACT 142

INTRODUCTION 142

METHODS 143

RESULTS 145

FUTURE WORK 145

TABELS AND FIGURES 146

$\begin{array}{ll}\text { REFERENCES } & 150\end{array}$

$\begin{array}{ll}\text { ACKNOWLEDGEMENT } & 151\end{array}$ 


\begin{abstract}
Aptamers are short single-stranded nucleic acids that can bind to their targets with high specificity and high affinity. To study aptamer function and dynamics, the malachite green aptamer was chosen as a model. Malachite green (MG) bleaching, in which an $\mathrm{OH}^{-}$attacks the central carbon (C1) of MG, was inhibited in the presence of the malachite green aptamer (MGA). The inhibition of MG bleaching by MGA could be reversed by an antisense oligonucleotide (AS) complementary to the MGA binding pocket. Computational cavity analysis of the NMR structure of the MGA-MG complex predicted that the $\mathrm{OH}^{-}$is sterically excluded from the $\mathrm{C} 1$ of MG. The prediction was confirmed experimentally using variants of the MGA with changes in the MG binding pocket. This work shows that molecular reactivity can be reversibly regulated by an aptamer-AS pair based on steric hindrance. In addition to demonstrate that aptamers could control molecular reactivity, aptamer dynamics was studied with a strategy combining molecular dynamics (MD) simulation and experimental verification. MD simulation predicted that the MG binding pocket of the MGA is largely pre-organized and that binding of MG involves reorganization of the pocket and a simultaneous twisting of the MGA terminal stems around the pocket. MD simulation also provided a 3D-structure model of unoccupied MGA that has not yet been obtained by biophysical measurements. These predictions were consistent with biochemical and biophysical measurements of the MGA-MG interaction including RNase I footprinting, melting curves, thermodynamic and kinetic constants measurement. This work shows that MD simulation can be used to extend our understanding of the dynamics of aptamer-target interaction which is not evident from static 3D-structures. To conclude, I have developed a novel concept to control molecular reactivity by an aptamer based on steric protection and a strategy to study the dynamics of aptamer-target interaction by combining MD simulation and experimental verification. The former has potential application in controlling metabolic reactions and protein modifications by small reactants and the latter may serve as a general
\end{abstract}


approach to study the dynamics of aptamer-target interaction for new insights into mechanisms of aptamer-target recognition. 


\section{CHAPTER 1. GENERAL INTRODUCTION \\ DISSERTATION ORGANIZATION}

This dissertation contains five chapters and an appendix.

Chapter 1 is a general introduction to the dissertation organization and the research aims and significance of the studies. The two research aims stated in chapter 1 are specifically addressed in chapters 3 and 4 .

Chapter 2 is a literature review of aptamer and the SELEX (Sequential Evolution of Ligands by Exponential Enrichment) procedure in general. Aptamers are first briefly introduced in comparison to chemicals and antibodies (Table1). Aptamer modifications (Fig. 1) are then described that are made to improve their pharmacokinetics and functionality. Multivalent aptamers are also discussed that have been made to 1) increase potency, 2) carry multiple functions and 3) have allosteric regulation (Table 3). SELEX, an in vitro technique to select aptamers, is then described in regard to its principle (Fig. 2) and technical details. A summary of 312 aptamer selection experiments is also presented (Table 2, Fig. 3).

Chapter 3 addresses the first research aim. In the first research aim, a novel concept is developed to control molecular reactivity by an aptamer based on steric hindrance. The concept is supported by a model in which the malachite green aptamer (MGA) is shown to control malachite green $(\mathrm{MG})$ bleaching. MG bleaching is a $\mathrm{pH}$ dependent chemical reaction in which an $\mathrm{OH}^{-}$attacks the central carbon (C1) of MG (Fig. 1,2). MG bleaching can be inhibited by the MGA, which can be reversed by an antisense oligonucleotide that targets the MGA binding pocket (Fig. 3). The mechanism by which MGA inhibits MG bleaching was predicted by a computational cavity analysis to be steric hindrance (Fig. 4). The computational prediction was confirmed by experimental analysis of MGA mutants with altered binding pockets (Fig 5, 6, 7).

The research described in chapter 3 is in a manuscript in preparation for submission to the Journal of Biological Chemistry. The contributions of the authors are described as follows. 
Tianjiao Wang designed experiments, collected and analyzed data (Figs. 1, 2, 3, 5, 6 and 7) and wrote the manuscript. Julie Hoy designed an experiment, collected and analyzed data for the experiment (Fig. 4) and edited the manuscript. Monica Lamm designed an experiment, collected and analyzed data for the experiment (MD simulation of MGA variants for cavity assay, in progress) and edited the manuscript. Marit Nilsen-Hamilton worked with Tianjiao Wang to design experiments, analyze the data and write the manuscript.

Chapter 4 addresses the second research aim. In the second research aim, molecular dynamics (MD) simulation with experimental validation was used to study the dynamics of aptamer-target interaction with the MGA-MG interaction as a model. MD simulation showed a combined preorgnization and induced-fit mechanism in the MGA-MG interaction and a twisting of the MGA upon MG binding (Fig 1.) There are structural discrepancies between simulated and experimental (NMR and crystal) structures (Table 1). The simulated structures of the MGA alone and the MGA-MG complex are more in line with the structures present under the buffer conditions used for binding studies as shown in RNase I footprinting (Fig. 2). The discrepancy between the simulated structure and the 3D-structure determined by NMR spectrometry and crystallography could be due to the different buffer conditions used for measurement. This is consistent with the differences in NMR spectra (Fig. 3) and the melting profiles (Fig. 4, Table 2) of the MGA and the MGA-MG complex in buffers that differed in $\mathrm{pH}$ and salt components and their concentrations. The prediction of a twisting of MGA upon MG binding was also confirmed with RNase I footprinting data of four MGA units linked tandemly with linkers (Fig. 5). The kinetic and thermodynamic constants were then measured to support a structural rearrangement during the MGA-MG interaction (Fig. 6, 7). Finally, a two-step mechanism of the MGA-MG interaction is proposed (Fig. 8).

The research work presented in chapter 4 is in preparation for submission to Journal of Molecular Biology. The contributions of the authors are described as follows. Tianjiao Wang designed experiments, collected and analyzed data (Fig. 1-8, Table 1-3) and wrote the manuscript. 
Monica Lamm designed an experiment, analyzed the data for the experiment (Fig.1) and edited the manuscript. Julie Hoy analyzed the data (Fig. 8) and edited the manuscript. Marit NilsenHamilton worked with Tianjiao Wang to design experiments, analyze the data and write the manuscript.

Chapter 5 is the general conclusion of the studies addressed in chapters 3 and 4 . This chapter summarizes 1) the results from studies of the effect of the MGA on MG hydroxylation that resulted in developing a new concept for controlling molecular reactivity by an aptamer (chapter 3) and 2) the results of studies to develop the application of MD simulation with experimental validation for studying the mechanism of an aptamer-target interaction (chapter 4). Other topics of discussion in chapter 5 are 1) future directions and potential applications of the insights achieved in chapter 3 and chapter 4. Finally, a perspective of aptamer research in general is discussed.

Appendix describes Tianjiao Wang's preliminary results from a SELEX procedure to select aptamers against the Lcn2 protein. The preliminary data is presented and future work is discussed.

\section{RESEARCH AIMS AND SIGNIFICANCE}

Aptamers are short nucleic acids that behave like antibodies but that have more dynamic structures. The dynamics in their structure allows the regulation of aptamer structure and function. Aptamers function mainly as binding partners and occasionally as catalysts. Understanding the molecular bases of aptamer functions and their dynamics is fundamental for effectively developing aptamer applications. Two specific aims have been pursued in this research work.

Aim 1: Controlling chemical reactivity by an aptamer. Chemical reactions in vivo are controlled by regulatory small molecules and proteins and by compartmentalization of the relevant enzyme(s). Various techniques have been developed to control chemical reactions in vivo by interfering with genes that encode enzymes or by interfering with an enzyme's function. 
These techniques include gene knockout, RNA interference, antisense oligonucleotides and chemical inhibitors $[1,2]$. By contrast, very few tools are available to control a chemical reaction in vivo by directly targeting the chemical reactants. Mutant enzymes that retain their ability to bind substrates but lose their ability for catalysis may be generated to compete with wild type enzymes for substrates but it is often difficult to generate such enzyme mutants [1]. Aptamers can encapsulate chemicals inside their binding pockets with high affinity and high specificity [3].

This leads to the hypothesis that an aptamer against a chemical reactant could sequester the reactant from other reactants in a chemical reaction, thus preventing the reaction from occurrence. This hypothesis is supported by the observation in chapter 3 that the MGA inhibits MG bleaching in vitro. This proof of the concept that chemical reactivity can be controlled by an aptamer in vitro provides a first step toward controlling chemical reactions in vivo by aptamers.

In vivo there are also spontaneous chemical reactions that don't involve any enzymes and reactions catalyzed by enzymes for which there is gene redundancy and that require knock-down of multiple genes to change the phenotype. In these situations aptamers could serve as unique tools to control chemical reactions at the reactant level. The concept of controlling chemical reactivity with aptamers has potential applications in vivo in controlling metabolism, protein modifications and the reactivity of endogenous toxic chemicals.

\section{Aim 2: Developing MD simulation as a tool to study aptamer-target interactions with}

experimental validation. Knowing the dynamics of aptamer-target interactions is fundamental to understanding many aptamer functions. Various experimental techniques have been developed to study aptamer structure and dynamics including single molecule measurement, NMR and structure probing (see references in chapter 4). Molecular dynamics (MD) simulation, on the other hand, can lead to hypotheses for experimental validation or extend the structure and dynamics information beyond the limitations of current experimental techniques. Although some MD simulations of aptamer-target interaction have been tried, they have not been tested rigorously against experimental data (see reference in chapter 4). The MGA-MG interaction was 
studied using MD simulation with experimental validation. The results showed that MD simulation could, with reasonable accuracy, extend structure and dynamics information beyond the 3D-structures of the MGA-MG determined by NMR spectrometry and the MGA-TMR determined by crystallography. These studies showed that the MD simulation could provide an experimentally verifiable model for the 3D-structure of an unoccupied MGA whose experimental 3D-structure has not been reported yet. There are more than 40 experimentally determined 3Dstructures of aptamer-target complexes and a few experimentally determined 3D-structures of unoccupied aptamers in the pdb database (http://www.pdb.org). This may be due to the dynamic nature of short nucleic acids that makes it difficult to collect high resolution data for 3D-structure determination of unoccupied aptamers by NMR spectrometry and crystallography. Thus MD simulation provides a tool to model the 3D-structure of an unoccupied aptamer for further experimental validation when the biophysically-determined structure for an aptamer is unavailable.

\section{REFERENCES}

1. Lindsay, M. A., Target discovery. Nat Rev Drug Discov, 2003. 2(10): p. 831-8.

2. Wang, S., T. B. Sim, Y. S. Kim, and Y. T. Chang, Tools for target identification and validation. Curr Opin Chem Biol, 2004. 8(4): p. 371-7.

3. Hermann, T. and D. J. Patel, Adaptive recognition by nucleic acid aptamers. Science, 2000. 287(5454): p. 820-5. 


\title{
CHAPTER 2. LITERATURE REVIEW
}

\author{
APTAMER \\ A nucleic acid aptamer is a single stranded DNA or RNA molecule that can bind to its target \\ with high affinity and high specificity. Peptide aptamers also exist with similar properties. However, \\ the term "aptamer" was coined to describe nucleic acid aptamers and peptide will not be discussed \\ here. Hereafter, the term "aptamer" refers exclusively to nucleic acid aptamers. \\ Aptamers were first selected in vitro and later found to exist in nature. There is 18 years of \\ history of aptamer science so far. An aptamer handbook has been published to describe various \\ aspects of aptamer research, development and application [1]. An aptamer database has also been \\ created to collect aptamers selected in vitro (http://aptamer.icmb.utexas.edu/) [2]. Natural aptamers \\ such as riboswitches can also be searched in RegRNA, a regulatory RNA motifs and elements \\ database (http://bidlab.life.nctu.edu.tw/RegRNA2/website/) [3]. \\ In many ways, aptamers can be considered as nucleic acid-type antibodies with properties \\ comparable with or even superior to conventional protein-type antibodies (Table 1). An aptamer \\ binds its target with the affinity in the $\mu \mathrm{M}$ to $\mathrm{pM}$ range. The binding of an aptmer to its target is very \\ specific. An aptamer can discriminate a single chemical group difference between two molecules or \\ even the same molecules with different conformations [4-6]. The affinity and specificity of aptamers \\ and antibodies are comparable. Aptamers have been selected in vitro for various targets (Table 2). \\ These targets range from small molecules to macromolecules and even to cells. Because aptamers are \\ selected in vitro, they are amenable to various chemical modifications, various selection conditions \\ and scale-up synthesis up to the kilogram level. In contrast to in vitro chemical synthesis and \\ selection for aptamers, antibodies are developed and produced by in vivo biological systems and \\ antigens for antibodies are usually limited to nontoxic macromolecules. So aptamers are superior to \\ antibodies in target ranges, in flexibility of selection and post-modifications and in cost. In addition,
}


aptamers can function both inside and outside cells [7-11] whereas antibodies usually only work outside cells to target secretory proteins or cell surface receptors. For therapy, aptamers have shown no intrinsic toxicity and immunogenicity [12-15] but antibodies, even humanized, could induce immunogenicity [16]. In sum, aptamers are competitive with or even superior to antibodies in many respects.

\section{APTAMER MODIFICATIONS}

Aptamers were originally selected from single stranded DNA or RNA molecules made of natural nucleotides. To increase functionality, affinity and nuclease resistance and to improve pharmacokinetics of aptamers, modifications have been introduced to different parts of aptamers including the nucleosides, the phosphodiester backbone and the 5' and 3'-termini (Figure 1). The modifications can be introduced either during SELEX or after SELEX or both. The modifications introduced during SELEX require compatible enzymology (T7 RNA polymerase, reverse transcriptase and DNA polymerase) and modifications introduced post-SELEX should be screened to avoid compromising aptamer function. Consistent efforts have been made to engineer enzymes to incorporate modified nucleotides efficiently $[17,18]$. These efforts will facilitate successful modifications during SELEX. So far, 100 different modifications can be readily introduced into aptamers at the 2'-position of ribose, the 5-position of pyrimines, the 8-position of purines, the 7position of 2'-deoxypurines, the oxygen of phosphodiester bond and others [19-22].

- To improve pharmacokinetics:

The pharmacokinetics of aptamers is determined by various properties of aptamers including their nuclease-resistance, their uptake and distribution in tissues and their circulation half-life in the blood of organisms. Modifications of aptamers can help to improve their pharmacokinetics for therapy and in vivo diagnosis.

Single or combinatorial modifications of aptamers with 2'-primary amines (2'- $\left.\mathrm{NH}_{2}\right), 2^{\prime}$ 'fluorides (2'-F) and 2'-O-methyl groups (2'-OME) have been done extensively as shown in Table 1. 
These modifications can be introduced with modified nucleotides either during SELEX or postSELEX. The 2'-F modified nucleotides, 2'F-dATP, 2'F-dCTP and 2'F-dGTP have been used in combination as substrates for thermostable DNA polymerases including Pfu (exo-), Vent(exo-), Deep Vent (exo-) and UITma [23]. Engineered T7 RNA polymerases have also been used to increase the efficiency of introducing modified nucleotides during SELEX. The Sousa group generated Y639F and Y639F/H784A T7 RNA polymerase mutants by rational design. The Y639 F variant can use 2'deoxy, 2'- $\mathrm{NH}_{2}$ and 2'-F NTPs as substrates while the $\mathrm{Y} 639 \mathrm{~F} / \mathrm{H} 784 \mathrm{~A}$ double mutant can further use NTPs with bulky 2'-substitutions (2'-OME, 2'- $\left.\mathrm{N}_{3}\right)$ [24,25]. With optimized conditions, the Y639F/H784A/(K378R) T7 RNA polymerase can incorporate all four 2'-OME-NTPs [26]. In addition, the Ellington group selected a Y639V/H784G/E593G/V685A T7 RNA polymerse (RGVG8.1 variant) by in vitro evolution. The variant can incorporate 2'-OME U, 2'-OME C and 2'-OME A but not 2'-OME G [27].

2'- $\mathrm{NH}_{2}, 2$ '- $\mathrm{F}$ and 2'-OME were introduced to increase the nuclease resistance of aptamers. The half lives of natural RNAs in human serum are from seconds to hours but those of modified aptamers are days (e.g. >> $96 \mathrm{hr}$ for the 2'-OME-VEGF aptamer). In addition to nuclease resistance, these modifications also increase the affinities of aptamers by increasing their thermostability and chemical diversity. Pagratis, et al, introduced 2'- $\mathrm{NH}_{2}$ and 2'-F deoxypyrimidines into KGF aptamers during SELEX. They demonstrated that the 2'-F modification is superior to the 2'- $\mathrm{NH}_{2}$ modification in providing the aptamer with better thermostability, affinity and bioactivity [28]. In another study, Kubik, et al, introduced 2'-F, 2'- $\mathrm{NH}_{2}$ and 2' $\mathrm{F} / \mathrm{NH}_{2}$ deoxypyrimdines in parallel into IFN- $\gamma$ aptamers during SELEX, and showed that the 2' $-\mathrm{NH}_{2}$ modified aptamer had 3-4 times better affinity than the 2'-F modified aptamer. They suggested that these modifications contributed to high affinity aptamers by providing chemical and structural diversity [29]. The 2'- $\mathrm{NH}_{2}-\mathrm{Py}-2$ '-OME-Pu-modified VEGF aptamer is a good example of combining modifications during and post-SELEX. A 2'- $\mathrm{NH}_{2}-\mathrm{Py}$ modified VEGF aptamer was first selected by SELEX. Then 10 out of 14 deoxyribopurines of the 
selected aptamer (24 nt) were replaced with 2'-OME deoxyribopurines. This 2'-OME modification increased the VEGF aptamer affinity by 17 fold due to its decrease in $\mathrm{k}_{\text {off }}$ [30]. Fully 2'-OME and 2'deoxy-Pu-2'-OME-Py modified aptamers have also been generated [26,31]. They have superior stability in plasma and after being autoclaved at $125^{\circ} \mathrm{C}$. The 2 '-OME modifications occur naturally and are not substrates of human DNA polymerase. So 2'-OME modifications are less likely to cause toxicity compared to the 2'-F and 2'- $\mathrm{NH}_{2}$ modifications. Besides, 2'-OME modifications are less expensive compared with 2'-F and 2'- $\mathrm{NH}_{2}$ modifications.

Another form of 2'-modification introduced post-SELEX is the locked nucleic acid (LNA) in which the 2'-O and 4'-C are linked by a methylene group. LNA has been introduced to the stem I of a tenascin binding aptamer, TTA1 that was also 2'-F-Py and 2'-OME modified. The LNA modification further increased the thermostability and plasma stability of TTA1. In addition, the LNA-modified TTA1 showed higher tumor uptake and blood retention [32]. In an LNA and DNA chimeric aptamer against an HIV-1 TAR RNA element, the half life of the modified aptamer was increased from $<<1 \mathrm{~h}$ to $>>20 \mathrm{~h}$ in $10 \%$ calf serum [33]. Combinatory screening of LNA and 2'OME modifications in the loop region of an aptamer against the HIV-1 TAR RNA element also identified three derivatives that showed about 10 times higher affinity than the parental aptamer. One of them could inhibit TAR dependent transcription [34].

The 4'-thio modification is another way to provide nuclease resistance for aptamers. PostSELEX modification of an NF- $\mathrm{B}$ aptamer in stem I and in loop region increased the aptamer's thermostability ( $\operatorname{Tm}$ from $34.8^{\circ} \mathrm{C}$ to $45.7^{\circ} \mathrm{C}$ ). At the same time, the nuclease resistance of the aptamer was increased by three-fold [35]. After 4'-thioCTP and 4'-thioUTP were introduced into a human $\alpha$-thrombin aptamer during SELEX, the half life of the thioaptamer in the presence of $50 \mathrm{pg} / \mu \mathrm{l}$ RNase A was $19.5 \mathrm{~h}$ while that of the natural aptamer was $22 \min [36]$.

Phosphodiester backbone modifications can also provide nuclease resistance for aptamers. One or two of the nonbridging oxygens on the phosphodiester backbone can be replaced by thiol 
groups. Phosphorothioate nucleotides can be incorporated into nucleic acids during SELEX with lower efficiency by T7 RNA polymerase, DNA polymerase and reverse transcriptase [37] but phosphorodithioate nucleotides can only be introduced through post-SELEX modification. The modified aptamer is called a thioaptamer and is nuclease-resistant. Ds-DNA thioaptamers made from dATP $\alpha$ S, dTTP, dCTP and dGTP have been selected for NF-IL6 [38], NFkB(p50, p60, RelA) [39,40] and RNase H domain of HIV RT [41] with Kds in the low nM range. The half life of the thioaptamer against NF-IL6 was increased from about $5 \mathrm{~min}$ to about $20 \mathrm{~min}$ at the treatment of $0.12 \mathrm{pg} / \mathrm{ml}$ DNase I [38]. A RNA aptamer made from ATP $\alpha$ S, UTP, CTP and GTP has also been selected for Venezuelan equine encephalitis virus capsid protein with the $\mathrm{Kd}$ of $7 \mathrm{nM}$ [42]. In addition, a fully phosphorothiolated RNA aptamer against bFGF was generated with the Kd of $1.8 \mathrm{nM}$ [43].

Althought thioaptamers are nuclease-resistant, they tend to be more sticky and may bind nonspecifically to other proteins. This needs to be fine-tuned for thioaptamers.

In addition to modifications at the nucleoside and the phosphodiester backbone, capping at the 5' and 3'-termini can also increase resistance of aptamers to exonucleases. The degradation of aptamers in human blood is largely due to 3 ' $\rightarrow$ 5' exonucleases. A commonly used 3'-capping of aptamers is an inverted thymidine ( 3 '-idT), which creates an additional 5'-end without a 3 '-end. The 3'-capping with 3'-idT can greatly increase aptamer stability in an organism's blood. The half-life of a PDGF DNA aptamer modified with 2'-F, 2'-OME and 3'-capping by 3'-idT was increased from $0.6 \mathrm{~h}$ to $8 \mathrm{~h}$ in $85 \%$ rat serum [44]. 3'-idT capping was also used to further protect a 2 '-F modified Factor IXa RNA aptamer. The modified aptamer could inhibit Factor IXa function and prolong the clotting time of human plasma in a dose-dependent manner [45]. 3'-biotin capping of a thrombin aptamer has also been reported. The half-life of the nonmodified thrombin-DNA aptamer was 5-10 min in fresh noncirculating mouse and rabbit blood and those of 3'-biotin and 3'-biotin-streptavidin capped thrombin aptamers were 40-160 min and >160 min respectively [46]. Because phosphorothioate DNA or RNA is nuclease-resistant, short phosphorothioate caps at the 5' and 3' 
ends can also improve aptamer stability [30]. A special way to cap the 5' and 3' ends is circularization. Multivalent circular DNA aptamers have been generated through building blocks using both thrombin and L-selectin aptamers as examples. The circular DNA aptamers have been shown to be nuclease resistant. The half life of a circular divalent thrombin DNA aptamer was increased from $15 \mathrm{~min}$ to $6.8-13.6 \mathrm{~h}$ in pooled human serum and from $36 \mathrm{~min}$ to $15.4-32.1 \mathrm{~h}$ in human plasma. The half life of a circular heterovalent thrombin-selectin DNA aptamer was increased from $4.8 \mathrm{~h}$ to $9.5 \mathrm{~h}$ in pooled human serum and from $16 \mathrm{~h}$ to $50 \mathrm{~h}$ in human plasma $[47,48]$. A circular RNA aptamer for streptavidin generated through self-ligating permutated intron-exon sequence has already been reported. The circular RNA aptamer can be produced in vitro as well as in vivo $[49,50]$.

A different concept of providing nuclease-resistance, called spiegelmers, is based on chirality [51]. D-aptamers are first selected against molecules which are chiral to the desired targets. According to the chiral principle, the chiral aptamer (L-apatmers) will recognize the desired targets. Because nucleases can only use natural D-DNA/RNA as substrates, the L-aptamers (spiegelmers) are nuclease-resistant. A limitation of this approach is the synthesis of chiral targets. For example, instead of an integral protein, only a small domain of a protein can be chemically synthesized as the D-form. Spiegelmers are stable in serum. For example, no obvious degradation was observed in human serum even after $60 \mathrm{~h}$ for a spiegelmer (L-RNA) that binds D-adenosine. On the contrary, the D-RNA was completely degraded in $24 \mathrm{~s}$. Examples of spiegelmers can be found in Table 2.

Nuclease-resistance is only one part of the story of aptamer pharmacokinetics. Uptake and distribution in tissues and circulation half-lives of aptamers are other important aspects of aptamer pharmacokinetics. Aptamers are relatively small (10-15 kDa, 30-45 nt) and can be easily cleared from human blood through the kidney and liver. To increase the circulation half lives of aptamers, tagging aptamers at the termini is often used. The most commonly used tag is polyethylene glycol (PEG). Pegylation usually has little effect on the affinities of aptamers but it can dramatically 
increase the circulation half life of aptamers, thus enhancing in vivo aptamer activity. In addition, pegylation can affect the tissue distribution of aptamers. The effect of pegylation has been studied on both the 2'-F modified RNA and ss-DNA L-selectin aptamers. The results showed that affinities of pegylated L-selectin aptamers decreased from little up to 30 times with the increase of the PEG molecule weight. Although the Kds of pegylated selectin aptamers were compromised, the plasma clearance rate of the pegylated selectin aptamers administered by tail vein injection in SpragueDawlet rat was dramatically decreased. For the 2'-F modified RNA selectin aptamer, the half life $\left(t_{1 / 2}\right)$ was 10 min for the nonpegylated aptamer, 60 min for the $20 \mathrm{KDa}$ PEG conjugate and 228 min for the $40 \mathrm{KDa}$ PEG conjugate. Correspondingly, the in vivo ability of the pegylated L-selectin aptamer to inhibit lymphocyte trafficking in SCID mice was increased by $\sim 3$ times for the $20 \mathrm{KDa}$ PEG conjugate and by 27 times for the $40 \mathrm{KDa}$ conjugate compared with the L-selectin aptamer without pegylation [52]. Pegylation of a TGF 32 aptamer has also been reported. The pegylation didn't affect the KDa but changed the pharmacokinetics of the TGF $\beta 2$ aptamer. Subconjuctivally injected into Dutch-belted rabbits, the TGF $\beta 2$ aptamer without PEG entered into rabbits' plasma rapidly and reached the maximal drug concentration $(\mathrm{Cmax})$ in $1 \mathrm{~h}$ with an elimination half life $\left(\mathrm{t}_{1 / 2}\right)$ of 2.4 h. The TGF 32 aptamer conjugated with $20 \mathrm{KDa}$ and $40 \mathrm{KDa}$ PEG entered the rabbits' plasma more slowly and reached the Cmax in 6 and $12 \mathrm{~h}$ respectively with $\mathrm{t}_{1 / 2} \mathrm{~s}$ of $7.4 \mathrm{~h}$ and $6.8 \mathrm{~h}$ respectively. PEG conjugation also increased the Cmax of the TGF $\beta 2$ aptamer by 20-100 times in plasma and 5-6 times in aqueous humor. In aqueous humor, the $40 \mathrm{KDa}$ PEGylated TGF $\beta 2$ apatmer had a $\mathrm{t}_{1 / 2}$ of 25.8 h [53]. PEGylation of a PDGF aptamer has also been attempted. A 40 KDa PEGylation didn't affect the Kd of the PDGF aptamer and the PEGylated aptamer showed a biphasic clearance pattern in rat plasma with $\mathrm{t}_{1 / 2} \mathrm{~s}$ of $32 \mathrm{~min}$ and 134 min after i.v. injection [44]. The pharmacokinetics of Macugen, a 40 KDa PEGylated VEGF aptamer, has also been studied thoroughly in Rhesus monkeys. Macugen showed $\mathrm{t}_{1 / 2} \mathrm{~s}$ of $9.3 \mathrm{~h}$ in plasma after intravenous injection, $12 \mathrm{~h}$ in plasma after subcutaneous injection [54] and $94 \mathrm{~h}$ in vitreous humor [12]. 
In addition to pegylation, tagging with lipids may also increase the circulation half lives of aptamers and tagging with cell-penetrating peptides may help tissue uptake of aptamers. A VEGF aptamer has been tagged with dialkylglycerol (DAG), which can anchor the aptamer on liposomes. The tagging with DAG decreased the aptamer's affinity by 1.6 times. The anchoring of DAG-tagged aptamer to liposomes through DAG further decreased the apatmer's affinity by about 5 times. The $\mathrm{t}_{1 / 2}$ in plasma in Sprague-Dawley rats after intravenous bolus administration was 49 min for the VEGF aptamer, $67 \mathrm{~min}$ for the DAG-VEGF aptamer and $113 \mathrm{~min}$ for the liposome-DAG-VEGF aptamer. The increase of circulation half life in vivo is correlated to the dramatically increased ability of the tagged VEGF aptamers to inhibit vascular permeability and angiogenesis in vivo [55]. Cholesterol is another choice of lipid tag. Tagging with cholesterol prolonged the circulation half life of antisense oligonucleotides but had the opposite effect in an aptamer study [56]. To study the effect of various factors on aptamer pharmacokinetics, an inactivated TGF $\beta 2$ aptamer was used. The study showed that the aptamers had circulation half lives of 0.6-16 h. PEGylation and aptamer sugar modifications played more important roles in aptamer pharmacokinetics and tissue distribution than the low molecular weight tags (cholesterol and cell-penetrating peptides). The aptamer abundance in plasma followed the trend of $20 \mathrm{KDa}$ PEG > 2'F, 2'OME mix > Arg7 > fully 2'OME. The $20 \mathrm{KDa}$ PEG could prevent renal clearance as well as the $40 \mathrm{KDa}$ PEG. The major uptake and distribution tissues for aptamers are the highly perfused organs (kidney and liver) and the mediastinal lymph nodes [56].

- To improve functionality

To improve pharmacokinetics is only one reason for aptamer modifications. Another reason for aptamer modification is to introduce new functionality. Unlike proteins composed of 20 different amino acids, nucleic acids are made from only four different nucleotides (A, G, C, T/U). To increase the numbers of functional groups and the complexity of nucleic acids, aminoacyl, hydrophobic, hydrophilic, charged and fluorescent modifications have been introduced into the 5-position of pyridine, the 8-position of purines and the 7-position of deoxyuridines during and post SELEX. 
For modifications during SELEX, the compatibility of the unnatural nucleotides with enzymes needs to be determined first. Compatibility depends on the types of modification, the polymerase and the nucleic acid sequence to be amplified or transcribed. UTP derivatives with hydrophobic and charged groups at the 5 position including phenyl, 4-pyridyl, 2-pyridyl, indolyl, isobutyl, imidazole and amino groups are as good substrates as natural nucleotides for T7 RNA polymerase [57]. For deoxynucleotide derivatives as substrates, in general the family B DNA polymerases (Vent, KOD Dash, Pwo) more efficiently use the modified substrates than the family A DNA polymerases (Taq, klenow, Tth). The Famulok group synthesized a series of C5, C7, C8 modified dATPs, dUTP, dCTPs and dGTPs with carboxyl, amino, amide and guanidinium groups. They tried to synthesize functionalized DNA enzymatically using multiple modified deoxynucleotides simultaneously as substrates. They found that the amount of the full length DNA synthesized by Pwo and Vent(exo-) DNA polymerases with multiple modified deoxynucleotides as substartes was $>70 \%$ of that with natural nucleotides as substrates [58]. The Kuwahara group synthesized 26 different dCTP and dUTP analogs that were modified at the C5 position by amino, hydroxyl, amide and guanidinium groups with different lengths of aliphatic linkers. They screened 260 different combinations of modified dCTP and dUTP, DNA polymerases and DNA templates to find the optimal compatibility of modified dCTP and dUTP with DNA polymerases and found that the substrate spectra followed the trend of Vent(exo-) $>$ KOD Dash/(exo-) $>$ Tth, Taq and the fidelity of incorporation followed the pattern of KOD Dash/(exo-) > Vent (exo-). They also showed that the efficiency of DNA enzymes to use modified dCTP and dUTP as substrates was affected by the modification chemistry, the linker length and the compositions of modified dCTP and dUTP [59]. The same group demonstrated that the KOD Dash DNA polymerase could efficiently use C5modified dUTP with amino acyl groups (arginyl, histdidyl, lysyl, phenylalanyl, tryptophanyl, lecucyl, prolyl, glutaminyl, seryl, O-benzyl seryl, threonyl, aspartyl and glutamyl cysteinyl) as substrates [60]. In addition to the $\mathrm{C} 5, \mathrm{C} 7$ and $\mathrm{C} 8$ modifications, N7- cyanoborane- dGTP was shown to be a good 
substrate for Taq and klenow (exo-) DNA polymerases [61]. Some DNA polymerases have also been engineered to extend their substrate spectra. For example, the Holliger group selected a Taq (M1) (G84A/D144G/K314R/E520G/F598L/A608V/E742G) by direct evolution with a compartmentalized self-replication method. The Taq (M1) can incorporate 7-deazaGTP, thiotriphospates and dNTPs labeled with rhodamine, fluorescein and biotin [62].

Because various modified nucleotides can be used as substrates for the synthesis of nucleic acids, modified nucleotides have been used in SELEX to increase the functionalities of aptamers. A 5-(1-pentynyl)-dUTP instead of TTP has been used to select thrombin DNA aptamers. The resulting aptamers showed a dramatic sequence difference from the aptamers selected with natural nucleotides. The 5-(1-pentynyl)-dU was also required for aptamer function [63]. Unlike 5-(1-pentynyl)-dUTP carrying a hydrophobic group, 5-(3"-aminopropynyl)-dUTP has a cationic functional group. An ATP aptamer has been selected with 5-(3"-aminopropynyl)-dU as one of its nucleosides. The selected aptamer also showed sequence differences from that selected using natural nucleotide triphosphate substrates [64]. A thymidine derivative ( $\mathrm{pppT}^{\mathrm{HM}}$ ) bearing an amino group with a positive charge has also been used in selecting a sialyllactose DNA aptamer. $\mathrm{T}^{\mathrm{HM}}$ was present at the three-way junction structures of the aptamer, which could be the binding pocket for sialyllactose and $\mathrm{T}^{\mathrm{HM}}$ might interact with the negatively charged sialyllactose [65].

Boron neutron capture therapy has been investigated for cancer therapy. Boron $\left({ }^{10} \mathrm{~B}\right) \mathrm{can}$ capture neutrons to form ${ }^{11} \mathrm{~B} .{ }^{11} \mathrm{~B}$ can then divide into ${ }^{4} \mathrm{He}$ and ${ }^{7} \mathrm{Li}$ and kills cells within a distance of 5-9 $\mu \mathrm{m}$. So, aptamers consisting of boronated nucleotides will gain new functionalities for cancer therapy if the aptamers are targeted to cancer markers. Aptamers against ATP have been selected using either 5' -( $\alpha$-P-borano)-UTP (bUTP) or 5'-( $\alpha$-P-borano)-GTP (bGTP) during SELEX experiments. The selected aptamers showed novel aptamer folding and the bU and bG were essential for aptamer function [66]. This provides an example of developing boronated aptamers. 
Fluorescence modifications have also been introduced to aptamers during and after SELEX to produce signaling aptamers. Several types of signaling aptamers have been reviewed [67]. One type of signaling aptamers has only a single fluorophore attached. The attached fluorophore changed its fluorescence intensity due to the structural reorganization of an aptamer upon ligand binding. Another type is called an aptamer beacon. In an aptamer beacon, ligand binding to its aptamer breaks the fluorphore and quencher pair at the 5' and 3' ends, thus resulting in increased fluorescence. A third type of signaling aptamer is called a structure-switching signaling aptamer. In this case, an aptamer is labeled with a fluorophore at one end and hybridized to an oligonucleotide with a quencher. Upon ligand binding, the hybridized oligonucleotide with quencher is then released and the fluorophore emission can be detected. In addition, some signaling aptamers generate signals based on fluorescence resonance energy transfer (FRET).

Another type of modification provides cross-linking ability by introducing 5-Br(d)U or 5I(d)U during SELEX. This type of aptamer is developed by SomaLogic Inc. for protein diagnosis and is called an photoaptamer $[68,69]$. The 5-BrUTP and 5-IUTP are good substrates for T7 RNA polymerase and the 5-BrdUTP requires the combination of Taq and Pwo DNA polymerases for efficient incorporation. Cross-linking only happens when an aptamer and a protein can be positioned closely enough with the right orientation. Under irradiation (308nm laser), 5-Br(d)U or 5-I(d)U can specifically cross-link with proximal amino acids having aromatic or sulfur groups. The cross-linked aptamer-protein complex can be washed with harsh conditions, thus providing superior specificity and signal/noise ratio.

Aptamers can also be used as delivery vehicles rather than sensors. Drugs or signaling molecules can be directly covalently linked to aptamers or indirectly through a hybridized oligonucleotide. Valine phosphonate (ValP), an irreversible inhibitor of neutrophil elastase, was linked to an oligonucleotide hybridized to a random DNA/RNA library. In vitro selection generated an aptamer-ValP complex with increased potency by three to four orders of magnitude and with better 
specificity compared to ValP alone [70,71]. An Ala-Ala-Pro-Val tetrapeptide, a weak inhibitor of human nutrophil elastase (hNE), was directly covalently linked to the 3'-end of a hNE aptamer with three 18-carbon linkers through post-SELEX modification. By this way, the aptamer could deliver the tetrapeptide to the active site of $\mathrm{hNE}$ and increase the drug potency by $10^{5}$ times [72]. In addition to delivering drugs, aptamers can be coupled to fluorescent molecules for flow cytometry [73] or to radioisotopes $\left({ }^{18} \mathrm{~F},{ }^{99 \mathrm{~m}} \mathrm{Tc},{ }^{123} \mathrm{I}\right)$ for imaging [74].

\section{MULTIVALENT APTAMERS}

Multivalent aptamers have been generated by rational design as well as by in vitro selection to increase potency, to carry multiple functions or for allosteric regulation.

\section{- To increase potency}

More units of an aptamer in a single molecule can capture more ligands, thus increasing aptamer potency. Expressing tobramycin and kanamycin aptamers inside E. coli could cause drugresistance and the resistance increased with the number of aptamer units in the expression vector. When inserted into the 5'-UTR, the multivalent tobramycin and kanamycin aptamers could also inhibit translation in the presence of tobramycin and kanamycin [10]. Multivalent aptamers have also been used to knock down protein function. A multivalent (12 pentavalent) B52 aptamer was expressed in cells and an organism (fly). The multivalent aptamer showed a 10-fold increase in affinity compared with the monovalent aptamer. Stable expression of the multivalent B52 aptamer inhibited B52 functions and reversed the phenotypes caused by overexpression of B52 [11]. Similarly, a multivalent thrombin aptamer showed stronger anticoagulant activity than a monovalent thrombin aptamer [47]. Another example is a tetravalent CTLA-4 aptamer. The T-cell receptor, CTLA-4, is a negative signal for T-cell activation. It exists as a dimer and is cross-linked by a pair of B7-1 proteins, which are its ligands. To inhibit CTLA-4 function, a CTL-4 aptamer was selected and its tetravalent form was tested and shown to be better able to inhibit CTLA-4 function than a monovalent form and thus enhance T cell immunity against tumors [75]. 
Divalent aptamers to cell surface receptors can stimulate signal transduction by inducing the dimerization normally caused by ligand binding. For example, a divalent aptamer against 4-1BB, a cell surface receptor on CD8+ T cells stimulate CD8+ T-cell activation while the monovalent 4-1BB aptamer was unable to do so [76].

Another purpose of constructing multivalent aptamers is to increase the signal and the resolution for imaging or diagnosis. A divalent human neutrophil elastase aptamer showed $\sim 20$ fold increase in affinity and stronger fluorescence compared with a monovalent form [73]. A molecule with multivalent malachite green aptamer was also found to bind more malachite green per molecule and emit a stronger fluorescence per molecule than a molecule with monovalent malachite green aptamer [77]. Another example is multivalent MS2 coat protein aptamers. Multivalent MS2 coat protein aptamers were inserted into the 3' end of lacZ mRNA. The inserted MS2 coat protein aptamers could capture coexpressed MS2-GFP fusion proteins to the lacZ mRNA, thus imaging lacZ mRNA movement in a cell. The results showed that individual lacZ mRNA movement inside a cell could be observed for lacZ mRNA containing 24 units of MS2 aptamers while only population resolution of lacZ mRNA movement inside a cell could be achieved for lacZ mRNA containing 6 and 12 units of MS2 aptamers [78].

- Multifunctional aptamers

To have different aptamers in a molecule carrying out multiple tasks has potential application in catalysis, biosensors and therapy. Some multifunctional aptamers have been reported. Burke DH [79] reported a general approach to generate bifunctional aptamers through chimeric SELEX. In the chimeric SELEX, a pool of CoA aptamer and a pool of chloramphenicol aptamer at two ends were linked through a homologous sequence in the middle. The linked pool was then reselected for its ability to bind to both CoA and chloramphenicol. The linked pool had a low affinity to both CoA and chloramphenicol at round 1 compared with the original CoA aptamer pool and the original chloramphenicol aptamer pool respectively. After five rounds of selection, bifunctional aptamers 
were generated from the linked pool. The selected bifunctional apatmers bound to both CoA and chloramphenicol with as good affinities as the original CoA and chloramphenicol individual pools. Instead of in vitro selection, rational design was also used to generate multifunctional aptamers. For example, a thrombin aptamer, an L-selectin aptamer and a DNA element conjugated to biotin or fluorescin were linked together through duplex linkers or three way junctions. The resulting multivalent aptamers had multiple functions including anticoagulation activity and application as biosensors [47,48]. Another bifunctional aptamer could sense either AMP or cocaine. The AMP and cocaine aptamer was linked into one piece, which was hydridized to another piece of DNA. Either AMP or cocaine could compete with the hybridized sequence and release the hybridized sequence to give colorimetric or electric signals for detection [80]. Yet another bivalent aptamer was also reported to bind simultaneously to the HIV transactivation responsive element (TAR) and the dimerization initiation site (DIS) in the 5' untranslated region. The two aptamers were linked through a PEG linker. The resulting bifunctional aptamer showed about 10 times higher affinity for HIV RNA 5 '-untranslated region compared with those of individual aptamers. The enhanced affinity was due to slower dissociation rate [81].

- Allosteric aptamers

Analogous to allosteric enzymes, allosteric aptamers refer to nucleic acids that have multiple domains for multiple targets. Binding of a target to its binding domain in an allosteric aptamer affects the binding of other targets to their corresponding domains. Various allosteric ribozymes have been generated by in vitro selection, rational design or the combination of both [82]. Allosteric aptamers can be developed with similar approaches. Allosteric aptamers also exist in nature. Some examples of allosteric aptamers are listed in Table 3. An allosteric aptamer can consist of as many as three domains: an effector module(s), communication module(s) and allosteric module(s). Binding of a target to an effector module transfers a signal to an allosteric module. The allostery mechanism can be i) An effector binding to the effector module stabilizes the communication module and thus the 
allosteric module, thus allowing a target to bind to the allosteric module [83-87] or ii) An effector binding to the effector module guides the proper folding of allosteric module for targte binding [88,89]. Allostery can be achieved through the change of dissociation rate [81].

\section{SELEX}

Systematic Evolution of Ligands by Exponential Enrichment (SELEX) is an in vitro technique to select aptamers and ribozymes using random nucleic acids libraries and in vitro selection and evolution technologies. The SELEX methodology was first developed in 1990 [90-92]. Since then, aptamers have been selected for various targets from small molecules (ions, small organic chemicals), macromolecules (proteins, carbohydrates and nucleic acids) to cells and tissues (Table 2). The SELEX methodology itself has evolved with time. Various SELEX formats have been developed beyond the conventional SELEX [93]. Some detailed protocols about conventional SELEX have been published that should be very helpful for novices for adapting this technology into their laboratories [94-99]. Here the focus of discussion is on the principle and technical issues of conventional SELEX.

The SELEX protocol consists of several steps (Figure 2). First, a random DNA pool of $10^{13}$ $10^{15}$ complexity needs to be synthesized. From the DNA pool, ds-DNAs are produced by either Klenow extension or polymerase chain reaction (PCR). The ds-DNAs then serve as templates to generate RNAs by in vitro transcription (for RNA-SELEX) or to generate ss-DNAs by strand separation (for DNA-SELEX). Next, iterative selection-amplificaton is carried out to enrich target binders. The ssDNAs or RNAs are first incubated with a target molecule. The target binders are then collected and the nonbinders are discarded. The ss-DNA binder or cDNA reversely transcribed from RNA binders are then amplified by PCR to generate ds-DNA for next selection-amplificaton cycle. The iterative selection-amplification steps will be done until no further enrichment can be achieved. This usually takes 2-20 cycles. Finally, the binders will be cloned and sequenced for further characterization. 
The principle and technical issues of conventional SELEX are discussed below.

1) Random pool and primers

The ss-DNA random pool for SELEX is usually synthesized at a $1 \mu \mathrm{mol}$ scale. This yields a complexity of $\sim 10^{15}$ different amplifiable sequences. As calculated from SELEX results, the probability of there existing an aptamer with high specificity and selectivity for its target ranges from $10^{-3}$ to $10^{-14}$ with mean and mode probability of $10^{-11}[100]$. In practice, a complexity of $10^{13}-10^{15}$ different ss-DNA is usually used in the initial pool in which each ss-DNA molecules consists of a random region in the middle and two constant regions at both ends for PCR amplification (Figure 2A). In DNA pool design, several factors need to be considered: pool length, degree of randomness and constant regions.

There are some debates about what is the optimal pool length for SELEX. On one hand, longer DNAs and RNAs are more likely to form more complex structures while keeping the smaller motifs present in shorter DNA and RNAs. On the other hand, extra sequences outside a motif may interfere with the correct folding of the motif. It has been suggested that longer random sequences (70-200 random positions) should be used to select rare activity (e.g. ribozymes) while shorter sequences ( $\leq 70$ random positions) can be used to select simple activity (e.g. aptamers) [101]. It has also been suggested that the optimal pool length is equal to $20 \mathrm{x}$ and $\mathrm{x}$ is the number of stems of an aptamer. This latter suggestion is based on the computational analysis of the secondary structure distribution of random pools according to graph theory [102]. In addition to computational analysis, an experiment has been designed to select an isoleucine aptamer from pools containing 16, 22, 2650 , 70 and 90 random residues. The results showed that the optimal random pool length for selecting an isoleucine aptamer is 50-70 nucleotides long [103]. In sum, ss-DNA pools with random positions $\leq$ 75 nucleotides are empirically used.

In addition to length, the degree of randomness is another factor to consider for pool design. Although most random pools used to select for novel binding are completely random, there are 
arguments that biased random pools should be designed. Computational analysis of the secondary structure distribution of completely random pools has shown that the pools tend to have simple motifs while more complex motifs are rare [102]. This argues that we may be able to select aptamers for better activities if we can design a pool to sample both simple and complex motifs. To meet this aim, a web server called Rag PooLs has been developed (http://rubin2.biomath.nyu.edu) [104,105]. The Rag PooLs server consists of two parts, pool designer and pool analyzer. In pool designer, users need to specify three inputs, RNA topology distribution, number of mixing matrices and starting sequences. There are 30 starting sequences that represent different topologies, lengths and functions and 34 mixing matrices that determine the degree of randomness in the database. Once the inputs are specified, the pool designer will calculate the optimal combination of starting sequences and mixing matrices as output. In pool analyzer, users can verify the structure distribution generated from the output at pool designer. Then the output from pool designer can be used to guide users' pool syntheisis. In sum, completely random pools are used to select most novel aptamers so far. It will be interesting to see the effect of biased random pool on aptamer selection in the future.

Another feature of pool design is the constant regions and the matching primers. Although constant regions have been shown to affect aptamer binding, the major concern about constant region design is the (RT-)PCR efficiency. The constant regions should not form stable secondary structures [103]. The matching primers should not have structural features that can cause PCR artifacts like primer dimers. Two features of the 3'-ends of primers can help to improve amplification efficiency. One is to have WSS ( $\mathrm{W}=\mathrm{A}$, or $\mathrm{T}, \mathrm{S}=\mathrm{C}$ or $\mathrm{G}$ ) to ensure good extension and the other is to have A/Trich regions to avoid mispriming [95]. For RNA-SELEX, a T7 promoter needs to be included in one of the primers. I prefer to have primers that work at relatively high temperatures $\left(65-70{ }^{\circ} \mathrm{C}\right)$ because aptamers often have stable secondary structures. Relatively high temperature $\left(65-70^{\circ} \mathrm{C}\right)$ helps to overcome stable secondary structures of aptamers for efficient (RT-)PCR amplification. Many 
successful random pools and primer sets have been reported that can be readily used or serve as references for design $[95,96]$.

In addition to the design concerns just mentioned, cross contamination of pool and primers should be avoided during synthesis or later SELEX experiments.

2) Targets

Aptamers have been selected for various targets. Theoretically, aptamers can be selected for any targets but it is not true in practice. Some targets may be just better than others in SELEX. According to Table 2, the number of aptamers selected for targets follows the trend of proteins > small organic molecules $>>$ peptides, nucleic acids $>$ carbohydrates $>$ ions, lipids. The affinity and specificity of a binder is often assumed to correlate to the rigidity of its target. Although this is not always true, the trend observed from Table 2 may reflect that in general the rigidity of a target may contribute to successful SELEX. There are two reports of aptamers binding to divalent ions. The affinity and specificity of these aptamers are not very good. This is expected because ions have too small surfaces to interact extensively with their aptamers. It has been shown that aptamers can bind to the aliphatic side chain of L-valine $\left[\mathrm{CH}\left(\mathrm{CH}_{3}\right)_{2}\right]$ with the $\mathrm{Kd}$ of $12 \mathrm{mM}$ [106] and to cholic acids with $\mathrm{Kd}$ of $6.4-67.5 \mu \mathrm{M}[107,108]$. It will be interesting to see whether aptamers can be developed more broadly to study lipids in the future.

Proteins have been the major targets for SELEX. This is partly due to the importance of protein functions and potential diagnostic and therapeutic applications of aptamers against proteins. Another reason is that proteins have large surfaces that allow extensive interactions between proteins and aptamers. Empirically, proteins with basic patches are considered to be good targets for SELEX [94]. This is exemplified by nucleic acids and nucleotides binding proteins and heparin-binding proteins such as thrombin and bFGF. Hydrophobic proteins on the other hand, are considered as poor targets for SELEX because nucleic acids have limited capacity for hydrophobic interactions [100]. The second largest class of targets is small organic molecules. Small organic molecules with 
heterocyclic rings and planar structures are considered as good targets for SELEX [109]. This is exemplified by aptamers for NTPs and their analogs, theophyline, histidine, tryptophan, tyrosine, phenylalaninamide, tetracyclines, porphyrin and organic dyes (Table 2). As mentioned above, small hydrophobic molecules are poor targets for SELEX.

In sum, although there are some empirical rules to indicate what targets may be good for SELEX, trial and error is used by scientist in practice.

3) Generation of ss-DNA or RNA

RNA for SELEX can be readily generated by in vitro transcription with T7 RNA polymerase. This can be done with commercially available kits (e.g. Ampliscribe T7-Flash from Epicentre) or using recombinant T7 RNA polymerase [24,25] prepared in a research lab. Complete digestion of dsDNA templates after in vitro transcription is an important step because residual ds-DNA from this step may be introduced to the (RT-) PCR step later. If this happens, unwanted amplification from the undigested ds-DNA will be carried forward and slow down or even fail SELEX. It is suggested to gel-purified RNA from in vitro transcription at every selection cycle. Usually 1-2 nmol RNA can be readily produced from a $20 \mu \mathrm{l}$ reaction with a 50-100 pmol ds-DNA template.

Single-stranded DNA templates for SELEX can be readily produced from denatured ds-DNA by streptavidin-bead capture or other means. For the same argument mentioned above, the quality of the ss-DNA needs to be checked to avoid ds-DNA being carried forward.

4) Target-nucleic acids incubations

There are several factors to consider for target-nucleic acids incubations. These include binding buffer, incubation time and temperature and selection stringency. Some mathematical models have been constructed to predict optimal conditions for SELEX [110,111]. Empirically, a SELEX can be started with low stringency to enrich binders. Once the enrichment of binders is oberserved, more stringent conditions are applied to select high affinity binders [94]. A rule of thumb is to choose a target concentration under which 5-10\% of ss-DNA or RNA will bind to the target. In 
practice, many targets may not have obvious binding to ss-DNA or RNA $(<1 \%)$ in the first round of selection. In this case, the starting concentration of proteins can be set to $1-10 \mu \mathrm{M}$ and that of small molecules to $\mathrm{mM}$ because aptamers against proteins usually have $\mathrm{Kds}$ of $\mathrm{nM}-\mathrm{pM}$ and aptamers against small molecules have Kds of $\mu \mathrm{M}$. The target/nucleic acids ratio can be set to 0.1-10.

Binding buffers: Usually a binding buffer consists of a buffering agent (Tris, HEPES, phosphate, pH 6-8), salts ( $\mathrm{NaCl}$ or $\mathrm{KCl}, 50-200 \mathrm{mM})$ and $\mathrm{Mg}^{2+}(1-10 \mathrm{mM})$. In practice, buffers are user-defined to meet their specific purposes. Aptamers have been selected in various binding solutions including organic solvents. An aptamer for bisphenol A has been selected in 2-50\% of either one or the mixture of dioxane, dimethyl sulfoxide, N,N-dimethylformamide, tetrahydrofuran, ethanol and methanol (USPTO\#: 20050282226). In addition, an FMN aptamer has been shown to function properly even in $80 \%$ acetonitrile [112].

Incubation time and temperature: Usually SELEX is carried out at either $25^{\circ} \mathrm{C}$ or $37{ }^{\circ} \mathrm{C}$. The incubation time varies according to the nature of targets. Longer incubation time helps rare high affinity binders to have chances to bind. Usually 5-30 min is sufficient for binding.

Selection stringency: Selection stringency is affected by many factors. These include the concentration and ratio of target/nucleic acids, competitors, dilution and washing. As mentioned above, it may be wise to start with low stringency until enrichment is observed. A binding assay is needed to monitor the SELEX in progress. Usually the binding assay is carried out every three cycles. 5) Separation

There are many ways to separate binders from nonbinders. These include nitrocellulose membrane filtration, affinity chromatography, electromobility shift assay, immunoprecipitation, centrifugation, flow cytometry, surface plasmon resonance and capillary electrophoresis (Table 2) [113]. For separation, there are two factors to consider; one is resolution and the other is nonspecific binding to a matrix. 
The most commonly used separation methods are nitrocellulose membrane filtration and affinity chromatography (Table 2). Both methods require matrices (membrane vs resin) for separation. Usually nonspecific binding of nucleic acids to matrices is $0.1-1 \%$ and the average probability of aptamers existing in a pool is $10^{-11}$. This may explain why SELEX needs multiple selection cycles to enrich aptamers. Nitrocellulose membrane filtration and affinity chromatography have their own advantages and disadvantages for selection. The advantage of nitrocellulose membrane filtration is that no coupling of proteins to matrices is required, thus fewer proteins are needed. The disadvantage is that it is usually used only for selecting RNA aptamers against proteins. Although there are some successful examples of ss-DNA aptamers selected with nitrocellulose membrane filtration, nitrocellulose membrane filtration in general is not good for selecting ss-DNA aptamers because ss-DNA molecules can be easily enriched as nitrocellulose membrane binders due to the hydrophobic property of ss-DNA molecules [99]. On the other hand, affinity chromatography can be used for selecting apatmers for broader range of targets than nitrocellulose membrane filtration. Compared to nitrocellulose membrane filtration, affinity chromatography usually requires much more target due to the need to couple the targets to affinity resins. Because of the high density of the target on the affinity matrix, it is more difficult to select very high affinity aptamers in affinity chromatography.

Capillary electrophoresis is well known for its high resolution in separation. Recently, capillary electrophoresis has been applied in SELEX as a means of separation of binders from nonbinders [114-121]. Usually it only takes 2-4 cycles to select an aptamer with capillary electrophoresis SELEX, compared to about 12 cycles in conventional SELEX. Capillary electrophoresis SELEX holds promise for developing highly efficient SELEX but it may not be applicable to situations in which the aptamer-target interaction is sensitive to electric fields.

6) Reverse transcription-polymerase chain reaction (RT-PCR) 
After binders are separated from nonbinders, they need to be amplified for the next selection cycle. This is done by PCR. RNA binders need to be reversely transcribed by a reverse transcriptase first to generate cDNAs for PCR. Various reverse transcriptases have been used in SELEX to generate cDNA from RNA binders but there is no report yet to compare the efficiency of reverse transcriptases on cDNA generation from RNA binders. Because aptamers usually have stable structures, I prefer reverse transcriptases that work at high temperature $\left(55-70{ }^{\circ} \mathrm{C}\right)$, such as SuperScript, ThermoScript and Thermo-X reverse transcriptase from Invitrogen.

Efficient PCR is also very important. An amplification efficiency of $90-100 \%$ (equivalent to 1.8-2 times amplification per DNA per cycle) is required to avoid biased amplification in PCR. It has been shown that PCR amplication of a random pool is very different from PCR amplification of a homogenous DNA. In PCR amplication of a random pool, the ds-DNA products reach a maximum after 15-18 PCR cycles while ss-ds DNA byproducts due to random hybridization of ds-DNA products begin to appear at around cycle 15 in the presence of excess amounts of primers. Overamplification ( $>20$ cycles) will cause the ds-DNA products to disappear. It has also been shown that increasing the Taq DNA polymerase concentrations in the range of $0.025-0.1 \mathrm{U} / \mathrm{ul}$ helps to increase the yield of ds-DNA products [122]. In our SELEX conditions, PCR is usually carried out for less than 15 PCR cycles and the PCR amplication yield and quality is monitored after 7-10 cycles. Although there are some reports that the addition of single-stranded DNA binding protein, DMSO and betaine helps to improve PCR quality $[123,124]$. We have found that the effect of these additions is not consistent in our SELEX PCR condition (data not shown), so we do not add them.

7) Number of iterative selection rounds needed for SELEX

The number of iterative rounds for selecting an aptamer depends on the target nature and the selection conditions. It takes 2-26 rounds to enrich an aptamer with mean and modal number of rounds of 10 and 12 respectively (Figure 3, summarized from Table 2). It has also been reported that the number of rounds to complete a SELEX experiment ranges from 7 to 22 with a mean of $12 \pm 4$ 
and a median of 12 in a summary of 26 successful SELEX experiments using proteins as targets [111]. The difference between these two sets of numbers is a function of two different, but overlapping data sets. In practice, a binding assay is carried out at about every three rounds to determine the SELEX progress. The selections are stopped when no further enrichment is observed. If various conditions have been tried and no obvious enrichment is observed repeatedly after 15 rounds of SELEX, then the selected target may not be suitable for SELEX.

8) Cloning, sequencing and further characterization of aptamers

After a SELEX experiment has been completed, the selected binders need to be cloned for sequencing and further characterization of aptamers. There are many ways to clone binders. I recommend TOPO TA cloning (Invitrogen), which is fast and efficient. High throughput plasmid preparation and high throughput sequencing can be done in several days. The sequencing results can be analyzed by alignment tools, e.g. Vector NTI and secondary structure prediction tools, e.g. Sfold. Minimization, functional and structural studies can be done by footprinting, NMR, x-ray crystallography among other techniques.

9) Reselection to improve affinity

If the affinity of an aptamer after selection is not satisfactory, reselection to improve its affinity can be done by doping [125] or nonhomologous random recombination [126]. It has been shown that nonhomologous random recombination is even more efficient than doping [126]. Doping involves partially randomizing an aptamer composition. It has been shown computationally that the doping percentage has a large effect on the sequence distribution in the pool, thus affecting the reselection results. A web tool for calculating the effect of doping rate is provided at http://bayes.colorado.edu/doped pools [125]. If an aptamer sequence is similar to a starting sequence in RagPool, RagPool may also be used as a guideline to choose an appropriate doping percentage $[104,105]$. 
In nonhomologous random recombination, ds-DNAs derived from imperfect aptamers are digested with DNase I randomly and the digested fragments are extended by T4 DNA polymerase to generate blunt-ends. The fragements with blunt-ends are then ligated with excess of a DNA hairpin to produce ligation products that have DNA hairpins at both ends. The ligation products are then treated with a restriction endonuclease to cleave off the loop regions of the hairpins. The resulting dsDNAs then serve as templates for the reselection pool [126]. Compared with doping, nonhomologous random recombination can provide more diversity of structures. This may explain why it can improve affinity even more than doping.

In summary, the principle and technical issues of conventional SELEX have been discussed. SELEX technology itself has also been evolving with time. A future challenge is to develop SELEX into an automated, highly efficient and high throughput methodology. Some efforts have been made toward this end. A SELEX procedure has been integrated into a robotic workstation to carry out automatic selection with modified vacuum filtration as a separation method [127]. The system can also be coupled to high throughput aptamer characterization including sequencing and affinity measurements [128]. To monitor the SELEX progress and to avoid overamplification of target binders, a fluorescence reader is also integrated into a robotic system [129]. A method combining in vitro transcription, translation and biotinylation has also been developed to generate biotinylated proteins for SELEX directly from their gene sequences [130]. It has also been proposed that proteins can be immobilized in 96-well plates through hydrophobic interactions, which is compatible with a robotic workstation [131]. Theoretically, 96 SELEXes can be finished in just 2 days in a robotic workstation integrated with 96-well plates immobilized with targets. In another automation system, a robotics is integrated with an affinity column, a PCR thermal cycler, an HPLC and a sample operation system [132]. Instead of using a workstation, another way to implement high throughput SELEX is through miniaturization. An automated microfluidic SELEX prototype has been reported [133]. This 
represents the first effort toward a microfabricated chip-based SELEX platform. Although automatic SELEX platforms have been attempted, they are not available commercially so far. 


\section{TABLES AND FIGURES}

Table 1. Comparison of chemicals, aptamers and antibodies

\begin{tabular}{|c|c|c|c|}
\hline & chemicals & aptamers & antibodies \\
\hline size & $<1 \mathrm{kDa}$ & $10-15 \mathrm{kDa}$ & $150 \mathrm{kDa}$ \\
\hline targets & proteins & $\begin{array}{l}\text { everything including } \\
\text { toxic targets (from ions } \\
\text { to macromolecules) }\end{array}$ & $\begin{array}{l}\text { haptens or } \\
\text { macromolecules }\end{array}$ \\
\hline affinity & $\mathrm{mM}-\mathrm{nM}$ & $\mu \mathrm{M}-\mathrm{pM}$ & nM-pM \\
\hline immunogenicity & $\begin{array}{l}\text { fit to a binding site } \\
\text { of a molecule }\end{array}$ & $\begin{array}{l}\text { distinguish even a single } \\
\text { chemical group } \\
\text { difference and } \\
\text { conformation difference } \\
\text { no }\end{array}$ & $\begin{array}{l}\text { distinguish even a } \\
\text { single chemical group } \\
\text { difference and } \\
\text { conformation difference } \\
\text { yes }\end{array}$ \\
\hline $\begin{array}{l}\text { tissue } \\
\text { penetration }\end{array}$ & $\begin{array}{l}\text { enter into tissues and } \\
\text { cells easily }\end{array}$ & $\begin{array}{l}\text { moderate ability to enter } \\
\text { into tissues and cells }\end{array}$ & $\begin{array}{l}\text { difficult to penetrate } \\
\text { into tissues and cells }\end{array}$ \\
\hline $\begin{array}{l}\text { function inside } \\
\text { cells }\end{array}$ & Yes & Yes & No \\
\hline antidotes & sometimes & $\begin{array}{l}\text { antisense } \\
\text { oligonucleotides }\end{array}$ & no \\
\hline $\begin{array}{l}\text { breaking the } \\
\text { interface } \\
\text { between } \\
\text { macromolecules }\end{array}$ & $\begin{array}{l}\text { difficult to break } \\
\text { extensive interface } \\
\text { between } \\
\text { macromolecules }\end{array}$ & $\begin{array}{l}\text { capable of blocking } \\
\text { macromolecule } \\
\text { interactions }\end{array}$ & $\begin{array}{l}\text { capable of blocking } \\
\text { macromolecule } \\
\text { interactions }\end{array}$ \\
\hline $\begin{array}{l}\text { screening } \\
\text { process }\end{array}$ & $\begin{array}{l}\text { in vitro rational } \\
\text { design or } \\
\text { combinatory } \\
\text { chemistry selection }\end{array}$ & $\begin{array}{l}\text { in vitro combinatory } \\
\text { chemistry selection }\end{array}$ & $\begin{array}{l}\text { in vivo selection in } \\
\text { biological systems }\end{array}$ \\
\hline $\begin{array}{l}\text { selection } \\
\text { conditions }\end{array}$ & various & various & physiological condition \\
\hline $\begin{array}{l}\text { chemical } \\
\text { modifications }\end{array}$ & various & various & limited \\
\hline Thermostability & stable & stable & temperature sensitive \\
\hline shelf life & unlimited & unlimited & $\sim 5$ years \\
\hline $\begin{array}{l}\text { biological half } \\
\text { life }\end{array}$ & minutes to days & hours to days & hours to days \\
\hline $\cos t$ & inexpensive & relatively expensive & very expensive \\
\hline
\end{tabular}


Table 2. Aptamer selected in vitro

\begin{tabular}{|c|c|c|c|c|}
\hline target & types & $\begin{array}{l}\text { Separation } \\
\text { methods (rounds) }\end{array}$ & $\mathrm{Kds}$ & $\begin{array}{l}\text { 3D-structures } \\
\text { (PDB ID) }\end{array}$ \\
\hline \multicolumn{5}{|l|}{ Ions } \\
\hline $\begin{array}{l}\text { divalent }\left(\mathrm{Zn}^{2+},\right. \\
\left.\mathrm{Ni}^{2+}, \mathrm{Co}^{2+}, \mathrm{Cd}^{2+}\right)\end{array}$ & RNA & $\begin{array}{l}\text { affinity chroma- } \\
\text { tography (7) }\end{array}$ & $1.2 \mathrm{mM}$ [134] & \\
\hline $\mathrm{Cd}^{2+}$ and $\mathrm{Ni} 2+$ & RNA & $\begin{array}{l}\text { affinity chroma- } \\
\text { tography (8) }\end{array}$ & $0.8-29 \mu \mathrm{M}[135]$ & \\
\hline \multicolumn{5}{|l|}{$\begin{array}{l}\text { Nucleotides and } \\
\text { cofactors }\end{array}$} \\
\hline $\begin{array}{l}\text { adenine or } \\
\text { adenosine }\end{array}$ & RNA & $\begin{array}{l}\text { affinity chroma- } \\
\text { tography }\end{array}$ & & \\
\hline \multirow[t]{12}{*}{ portions } & & ATP/AMP- (8) & $0.7-50 \mu \mathrm{M}[136]$ & 1RAW [158] \\
\hline & & & & $1 \mathrm{AMO}$ [159] \\
\hline & & SAM- (7) & N.D. [137] & \\
\hline & & SAH- (14) & 50-100 nM [138] & \\
\hline & & FMN/FAD - $(7,5)$ & $0.5-1.4 \mu \mathrm{M}[139]$ & \\
\hline & & CoA- $(8,13,7$, & $0.5-2.4 \mu \mathrm{M}$ & \\
\hline & & 12) & {$[140,141]$} & \\
\hline & & cAMP-(5) & $10 \mu \mathrm{M}[142]$ & \\
\hline & & adenine- (12) & $10 \mu \mathrm{M}[143]$ & \\
\hline & boronated-RNA & ATP/AMP- (8) & $10 \mu \mathrm{M}[66]$ & \\
\hline & DNA & ATP-(8) & $6 \mu \mathrm{M}[144]$ & 1AW4 [160] \\
\hline & L-RNA & adenosine (5) & $1.8 \mu \mathrm{M}[145]$ & \\
\hline biotin & RNA & $\begin{array}{l}\text { affinity chroma- } \\
\text { tography (7) }\end{array}$ & $5.7 \mu \mathrm{M}[146,147]$ & $1 \mathrm{~F} 27[161] *$ \\
\hline FAD and FMN & RNA & $\begin{array}{l}\text { affinity chroma- } \\
\text { tography }(8,9)\end{array}$ & $\begin{array}{l}0.5-50 \mu \mathrm{M} \\
{[139,148,149]}\end{array}$ & $1 F M N$ [162] \\
\hline GTP & RNA & $\begin{array}{l}\text { affinity chroma- } \\
\text { tography (10) }\end{array}$ & 25-500 nM [150] & 2AU4 [163] \\
\hline $\begin{array}{l}\mathrm{m}^{7}-\mathrm{GTP}, \mathrm{m}^{7}- \\
\text { GDP, } \mathrm{m}^{7} \mathrm{GpppG} \\
\text { and } \mathrm{m}^{7} \mathrm{GpppA}\end{array}$ & RNA & $\begin{array}{l}\text { affinity chroma- } \\
\text { tography (8) }\end{array}$ & $0.5-2 \mu \mathrm{M}[151]$ & \\
\hline NMN and NAD & RNA & $\begin{array}{l}\text { affinity chroma- } \\
\text { tography }(8)\end{array}$ & $2.5 \mu \mathrm{M}[148]$ & \\
\hline VB12 & RNA & $\begin{array}{l}\text { affinity chroma- } \\
\text { tography }(8)\end{array}$ & $\begin{array}{l}0.09-8.8 \mu \mathrm{M} \\
{[152]}\end{array}$ & $\begin{array}{l}\text { 1DDY [164] * } \\
\text { 1ET4 [165] * }\end{array}$ \\
\hline $\begin{array}{l}\text { xanthine and } \\
\text { guanine }\end{array}$ & RNA & $\begin{array}{l}\text { affinity chroma- } \\
\text { tography (5) }\end{array}$ & $3.3 \mu \mathrm{M}[153]$ & \\
\hline \multicolumn{5}{|l|}{ Amino acids } \\
\hline D-tryptophan & RNA & $\begin{array}{l}\text { affinity chroma- } \\
\text { tography (7) }\end{array}$ & $18 \mu \mathrm{M}[154]$ & \\
\hline $\begin{array}{l}\mathrm{D} / \mathrm{L} \text {-arginine and } \\
\text { argininamide }\end{array}$ & DNA & $\begin{array}{l}\text { affinity chroma- } \\
\text { tography (12) }\end{array}$ & $0.1-2.5 \mathrm{mM}$ [168] & $\begin{array}{l}\text { 1OLD [184] } \\
\text { 2ARG[185] }\end{array}$ \\
\hline L-arginine & L-RNA & $\begin{array}{l}\text { affinity chroma- } \\
\text { tography (11) }\end{array}$ & $129 \mu \mathrm{M}[167]$ & 1DB6 [186] \\
\hline
\end{tabular}


Table 2. Aptamer selected in vitro (continued)

\begin{tabular}{|c|c|c|c|c|}
\hline targets & types & $\begin{array}{l}\text { Separation } \\
\text { methods (rounds) }\end{array}$ & $\mathrm{Kds}$ & $\begin{array}{l}\text { 3D-structures } \\
\text { (PDB ID) }\end{array}$ \\
\hline \multirow[t]{5}{*}{ L-arginine } & RNA & $\begin{array}{l}\text { affinity chroma- } \\
\text { tography }\end{array}$ & & \\
\hline & & (7) & $0.2-1 \mathrm{mM}$ [155] & \\
\hline & & (11) & $60 \mu \mathrm{M}[156]$ & $1 \mathrm{KOC}[166]$ \\
\hline & & (20) & $0.33 \mu \mathrm{M}[6]$ & \\
\hline & & (9) & $1.4-48 \mathrm{mM}$ [157] & \\
\hline L-citrulline & RNA & $\begin{array}{l}\text { affinity chroma- } \\
\text { tography (7) }\end{array}$ & $62-68 \mu \mathrm{M}[156]$ & 1KOD [166] \\
\hline L-histidine & RNA & $\begin{array}{l}\text { affinity chroma- } \\
\text { tography (6-7) }\end{array}$ & $8-54 \mu \mathrm{M}[169]$ & \\
\hline L-isoleculine & RNA & $\begin{array}{l}\text { affinity chroma- } \\
\text { tography (15) }\end{array}$ & $0.2-0.5 \mu \mathrm{M}[170]$ & \\
\hline $\begin{array}{l}\text { L-tyrosine and L- } \\
\text { tryptophan }\end{array}$ & RNA & $\begin{array}{l}\text { affinity chroma- } \\
\text { tography (13) }\end{array}$ & $35-743 \mu \mathrm{M}[171]$ & \\
\hline $\begin{array}{l}\text { L-tyrosinamide } \\
\text { and L- } \\
\text { phenylalaninamide }\end{array}$ & DNA & $\begin{array}{l}\text { affinity chroma- } \\
\text { tography (15) }\end{array}$ & 45-80 $\mu \mathrm{M}[172]$ & \\
\hline L-valine & RNA & $\begin{array}{l}\text { affinity chroma- } \\
\text { tography (9) }\end{array}$ & $12 \mathrm{mM}$ [106] & \\
\hline \multicolumn{5}{|l|}{ Antibiotics } \\
\hline chloramphenicol & RNA & $\begin{array}{l}\text { affinity chroma- } \\
\text { tography (12) }\end{array}$ & $2.1-270 \mu \mathrm{M}[173]$ & \\
\hline kanamycin $\mathrm{A} / \mathrm{B}$ & RNA & $\begin{array}{l}\text { affinity chroma- } \\
\text { tography }(4,14 \text {, } \\
12)\end{array}$ & $\begin{array}{l}0.18 \mu \mathrm{M} \\
{[10,174,175]}\end{array}$ & \\
\hline lividomycin & RNA & $\begin{array}{l}\text { affinity chroma- } \\
\text { tography (4) }\end{array}$ & $0.22 \mu \mathrm{M}[175]$ & \\
\hline moenomycin A & 2'- $\mathrm{NH}_{2}$-Py-RNA & $\begin{array}{l}\text { affinity chroma- } \\
\text { tography (12) }\end{array}$ & $0.3-0.4 \mu \mathrm{M}[176]$ & \\
\hline neomycin & RNA & $\begin{array}{l}\text { affinity chroma- } \\
\text { tography (8) }\end{array}$ & $0.1 \mu \mathrm{M}[177]$ & 1NEM [187] \\
\hline streptomycin & RNA & $\begin{array}{l}\text { affinity chroma- } \\
\text { tography (7) }\end{array}$ & $1 \mu \mathrm{M}[178]$ & $\begin{array}{l}\text { 1NTA and 1NTB } \\
{[188] *}\end{array}$ \\
\hline tetracycline & RNA & $\begin{array}{l}\text { affinity chroma- } \\
\text { tography (12) }\end{array}$ & $\begin{array}{l}1 \mu \mathrm{M}-0.77 \mathrm{nM} \\
{[179,180]}\end{array}$ & \\
\hline tobramycin & RNA & $\begin{array}{l}\text { affinity chroma- } \\
\text { tography }(6,13)\end{array}$ & $8 \mu \mathrm{M}-2 \mathrm{nM}[181]$ & $\begin{array}{l}\text { 1TOB [189] } \\
\text { 2TOB [190] }\end{array}$ \\
\hline viomycin & RNA & $\begin{array}{l}\text { affinity chroma- } \\
\text { tography (7) }\end{array}$ & $11-21 \mu \mathrm{M}[182]$ & \\
\hline
\end{tabular}

\section{Organic or}

fluorescent dyes

cibacron blue 3GA

RNA and DNA

affinity chroma- $\quad<0.1 \mathrm{mM}[92]$

tography $(6,5)$ 
Table 2. Aptamer selected in vitro (continued)

\begin{tabular}{|c|c|c|c|c|}
\hline targets & types & $\begin{array}{l}\text { Separation methods } \\
\text { (rounds) }\end{array}$ & Kds & $\begin{array}{l}\text { 3D-structures } \\
\text { (PDB ID) }\end{array}$ \\
\hline fluorescin & RNA & $\begin{array}{l}\text { affinity chroma- } \\
\text { tography }(6,5)\end{array}$ & N.D. [183] & \\
\hline Hoechst dye 33258 & RNA & $\begin{array}{l}\text { affinity chroma- } \\
\text { tography (10) }\end{array}$ & N.D. [10] & \\
\hline Hoechst derivative $7 \mathrm{e}$ & DNA & $\begin{array}{l}\text { affinity chroma- } \\
\text { tography (9) }\end{array}$ & $0.88 \mu \mathrm{M}[86]$ & \\
\hline malachite green & RNA & $\begin{array}{l}\text { affinity chroma- } \\
\text { tography (8) }\end{array}$ & $1 \mu \mathrm{M}[191]$ & $\begin{array}{l}\text { 1F1T [207] * } \\
\text { 1Q8N [208] }\end{array}$ \\
\hline reactive red 120 & RNA & $\begin{array}{l}\text { affinity chroma- } \\
\text { tography (6) }\end{array}$ & N.D. [92] & \\
\hline reactive yellow 86 & RNA & $\begin{array}{l}\text { affinity chroma- } \\
\text { tography (6) }\end{array}$ & N.D. [92] & \\
\hline reactive browm 10 & RNA & $\begin{array}{l}\text { affinity chroma- } \\
\text { tography (6) }\end{array}$ & N.D. [92] & \\
\hline \multirow[t]{2}{*}{ reactive green 19} & RNA & affinity chroma- & $0.6 \mathrm{nM}[92]$ & \\
\hline & DNA & tography $(6,6)$ & $33 \mu \mathrm{M}[192]$ & \\
\hline reactive blue 4 & RNA and DNA & $\begin{array}{l}\text { affinity chroma- } \\
\text { tography }(6,5)\end{array}$ & N.D. $[92,192]$ & \\
\hline \multirow[t]{2}{*}{ sulforhodamine B } & DNA & $\begin{array}{l}\text { affinity chroma- } \\
\text { tography (8) }\end{array}$ & $0.66 \mu \mathrm{M}[193]$ & \\
\hline & RNA & $\begin{array}{l}\text { affinity chroma- } \\
\text { tography (7) }\end{array}$ & $0.31 \mu \mathrm{M}[183]$ & \\
\hline \multicolumn{5}{|l|}{ Other small organics } \\
\hline CCdApPuro & RNA & $\begin{array}{l}\text { affinity chroma- } \\
\text { tography (10) }\end{array}$ & 10 nM [194] & \\
\hline cocaine & DNA & N/A & $\begin{array}{l}0.4-10 \mu \mathrm{M} \\
{[195,196]}\end{array}$ & \\
\hline codeine & RNA & $\begin{array}{l}\text { affinity chroma- } \\
\text { tography (15) }\end{array}$ & 2.5, $4 \mu \mathrm{M}[197]$ & \\
\hline $\begin{array}{l}\text { daunomycin and } \\
\text { doxorubicin }\end{array}$ & DNA & $\begin{array}{l}\text { affinity magnetic } \\
\text { beads (10) }\end{array}$ & $20 \mathrm{nM}[198]$ & \\
\hline dopamine & RNA & $\begin{array}{l}\text { affinity magnetic } \\
\text { beads (9) }\end{array}$ & $1.6 \mu \mathrm{M}[199]$ & \\
\hline ethanolamine & DNA & $\begin{array}{l}\text { affinity magnetic } \\
\text { beads (10) }\end{array}$ & 6-19 nM [200] & \\
\hline hemaptoporphyrin IX & DNA & $\begin{array}{l}\text { affinity chroma- } \\
\text { tography }(7-10)\end{array}$ & $\begin{array}{l}22 \mathrm{mM}-1.6 \mu \mathrm{M} \\
{[201]}\end{array}$ & \\
\hline $\begin{array}{l}4,4^{\prime}- \\
\text { methylenedianiline }\end{array}$ & RNA & $\begin{array}{l}\text { affinity magnetic } \\
\text { beads (9) }\end{array}$ & $0.45-12 \mu \mathrm{M}[202]$ & \\
\hline porphyrins & DNA & $\begin{array}{l}\text { affinity chroma- } \\
\text { tography (12) }\end{array}$ & $\begin{array}{l}0.5-4.9 \mu \mathrm{M}[203- \\
205]\end{array}$ & \\
\hline theophylline & RNA & $\begin{array}{l}\text { affinity chroma- } \\
\text { tography (8) }\end{array}$ & $0.1 \mu \mathrm{M}[4]$ & $\begin{array}{l}\text { 1EHT [209] } \\
1 \mathrm{O} 15 \text { [210] }\end{array}$ \\
\hline thyroxin & RNA & $\begin{array}{l}\text { affinity chroma- } \\
\text { tography (12) }\end{array}$ & $50 \mu \mathrm{M}[206]$ & \\
\hline
\end{tabular}


Table 2. Aptamer selected in vitro (continued)

\begin{tabular}{|c|c|c|c|c|}
\hline targets & types & $\begin{array}{l}\text { Separation methods } \\
\text { (rounds) }\end{array}$ & $\mathrm{Kds}$ & $\begin{array}{l}\text { 3D-structures } \\
\text { (PDB ID) }\end{array}$ \\
\hline \multicolumn{5}{|l|}{ Carbohydrates } \\
\hline $\begin{array}{l}\text { cellobiose and } \\
\text { cellulose }\end{array}$ & DNA & $\begin{array}{l}\text { affinity chroma- } \\
\text { tography (14) }\end{array}$ & $1-100 \mu \mathrm{M}[211]$ & \\
\hline chitin & DNA & $\begin{array}{l}\text { affinity chroma- } \\
\text { tography (8) }\end{array}$ & N.D. [212] & \\
\hline $\operatorname{sLe}^{\mathrm{x}}$ & RNA & $\begin{array}{l}\text { affinity chroma- } \\
\text { tography (17) }\end{array}$ & $\begin{array}{l}0.085-10 \mathrm{nM} \\
{[213]}\end{array}$ & \\
\hline sialyllactose & $\mathrm{T}^{\mathrm{HM}}$-DNA & $\begin{array}{l}\text { Sialyllactose- } \\
\text { biotin-streptavidin } \\
\text { magnetic beads } \\
\text { (13) }\end{array}$ & $4.9 \mu \mathrm{M}[65]$ & \\
\hline sephadex G-100 & RNA & $\begin{array}{l}\text { affinity chroma- } \\
\text { tography (11) }\end{array}$ & N.D. [214] & \\
\hline \multicolumn{5}{|l|}{ Llipids } \\
\hline cholic acids & DNA & $\begin{array}{l}\text { affinity chroma- } \\
\text { tography (13) }\end{array}$ & $\begin{array}{l}6.4-67.5 \mu \mathrm{M} \\
{[107,108]}\end{array}$ & \\
\hline \multicolumn{5}{|l|}{ Peptides } \\
\hline $\begin{array}{l}\text { amyloid peptide } \beta \text { A4 } \\
(1-40) / \text { fibril }\end{array}$ & RNA & $\begin{array}{l}\text { affinity chroma- } \\
\text { tography ( } 8)\end{array}$ & 29-48 nM [215] & \\
\hline ARM model peptide & RNA & $\begin{array}{l}\text { quartz-crystal } \\
\text { microbalance }(7)\end{array}$ & $10 \mathrm{nM}[216]$ & \\
\hline$\alpha$-CGRP & L-RNA & $\begin{array}{l}\text { affinity chroma- } \\
\text { tography (15) }\end{array}$ & $2.5 \mathrm{nM}[217]$ & \\
\hline $\begin{array}{l}\text { farnesylated Ras } \\
\text { peptide }\end{array}$ & RNA & $\begin{array}{l}\text { affinity chroma- } \\
\text { tography (10) }\end{array}$ & $\begin{array}{l}0.14-0.93 \mu \mathrm{M} \\
{[218]}\end{array}$ & \\
\hline ghrelin & L-RNA & $\begin{array}{l}\text { affinity chroma- } \\
\text { tography }(17,18)\end{array}$ & $\begin{array}{l}45-90 \mathrm{nM}[219] \\
4.5-20 \mathrm{nM}[220]\end{array}$ & \\
\hline \multirow[t]{2}{*}{ GnRH } & L-DNA & $\begin{array}{l}\text { affinity chroma- } \\
\text { tography }(8,12)\end{array}$ & $\begin{array}{l}20-55 \mathrm{nM} \\
{[15,221]}\end{array}$ & \\
\hline & L-RNA & $\begin{array}{l}\text { affinity chroma- } \\
\text { tography (6) }\end{array}$ & $92 \mathrm{nM}[221]$ & \\
\hline MUC1 (peptides) & DNA & $\begin{array}{l}\text { affinity chroma- } \\
\text { tography (10) }\end{array}$ & $0.1-34 \mathrm{nM}[222]$ & \\
\hline N/OFQ & L-RNA & $\begin{array}{l}\text { affinity chroma- } \\
\text { tography (13) }\end{array}$ & $0.3-3 \mu \mathrm{M}[223]$ & \\
\hline \multirow[t]{2}{*}{ neuropeptide $\mathrm{Y}$} & DNA & $\begin{array}{l}\text { capillary } \\
\text { electrophoresis (4) }\end{array}$ & $0.3-1 \mu \mathrm{M}[118]$ & \\
\hline & $\begin{array}{l}2^{\prime}-\mathrm{NH}_{2}-\mathrm{Py}- \\
\text { RNA }\end{array}$ & $\begin{array}{l}\text { affinity chroma- } \\
\text { tography (12) }\end{array}$ & $0.4 \mu \mathrm{M}[224]$ & \\
\hline $\operatorname{PrP}^{\mathrm{C}}$-peptide (90-129) & $\begin{array}{l}2^{\prime}-\mathrm{NH}_{2}-\mathrm{Py}- \\
\text { RNA }\end{array}$ & $\begin{array}{l}\text { affinity chroma- } \\
\text { tography (12) }\end{array}$ & $0.1-10 \mu \mathrm{M}[225]$ & \\
\hline
\end{tabular}


Table 2. Aptamer selected in vitro (continued)

\begin{tabular}{|c|c|c|c|c|}
\hline targets & types & $\begin{array}{l}\text { Separation methods } \\
\text { (rounds) }\end{array}$ & $\mathrm{Kds}$ & $\begin{array}{l}\text { 3D-structures } \\
\text { (PDB ID) }\end{array}$ \\
\hline substance $\mathrm{P}$ & RNA & $\begin{array}{l}\text { affinity chroma- } \\
\text { tography (24) }\end{array}$ & $0.19-2 \mu \mathrm{M}[226]$ & \\
\hline vasopressin & L-DNA & $\begin{array}{l}\text { affinity chroma- } \\
\text { tography (13) }\end{array}$ & $1.2 \mu \mathrm{M}$ [227] & \\
\hline microcystin-LR & RNA & $\begin{array}{l}\text { affinity chroma- } \\
\text { tography (13) }\end{array}$ & $>1 \mu \mathrm{M}[228]$ & \\
\hline angiotensin I & DNA & $\begin{array}{l}\text { affinity chroma- } \\
\text { tography (9) }\end{array}$ & $0.1-0.5 \mu \mathrm{M}$ [229] & \\
\hline
\end{tabular}

\section{Proteins}

Antibodies

anti-g10 antibodies RNA

Immuno-

precipitation (3)

anti-NES Ab and RNA

affinity magnetic

CRM1/exportin 1

mAb 20 (anti-insulin RNA

receptor antibody)

2'- $\mathrm{NH}_{2}$-Py-RNA

beads (5)

affinity magnetic $\quad 2 \mathrm{nM}[233]$

beads (11)

magnetic affinity $\quad 30 \mathrm{nM}[234]$

column (12)

mAb 198 (anti-

AChR antibody)

2'- $\mathrm{NH}_{2}$-Py-RNA

Immuno-

6-60 nM

precipitation (12) $\quad[235,236]$

2'-F-Py-RNA

Immuno-

$25 \mathrm{nM}[237]$

precipitation (11)

mAb C595 (anti- DNA

MUC1)

Ig E

immobilization on

$0.05-0.67 \mu \mathrm{M}$

PCR-tube (10)

[238]

2'- $\mathrm{NH}_{2}-\mathrm{Py}-\mathrm{RNA} \quad$ filtration (9) $\quad 30 \mathrm{nM}$ [239]

DNA filtration (15) $10 \mathrm{nM}$ [239]

DNA capillary electro- 29 nM [116,117]

phoresis (4)

mAb G6-9 RNA

affinity chroma- $2 \mathrm{nM}$ [240]

tography (22)

Cell surface proteins

4-1BB (murine)

cytotoxic $\mathrm{T}$ cell

2'-F-Py-RNA

filtration (12)

$40 \mathrm{nM}[76]$

antigen 4

sCD4 (rat)

2'-F-Py-RNA

filtration (9)

$10 \mathrm{nM}$ [75]

$\beta 2$ integrin LFA-1 RNA

$(\alpha \mathrm{L} \beta 2$,

affinity chroma- $\quad$ low nM [241]

tography (15)

affinity chroma- $\quad 0.5-1 \mu \mathrm{M}$ [9]

tography (11)

CD11a/CD18)

DC-SIGN

DNA

microtiter plate

$22-50 \mathrm{nM}[242]$

(11) 
Table 2. Aptamer selected in vitro (continued)

\begin{tabular}{|c|c|c|c|c|}
\hline targets & types & $\begin{array}{l}\text { Separation methods } \\
\text { (rounds) }\end{array}$ & $\mathrm{Kds}$ & $\begin{array}{l}\text { 3D-structures } \\
\text { (PDB ID) }\end{array}$ \\
\hline Fibronectin (RGD) & DNA & $\begin{array}{l}\text { affinity chroma- } \\
\text { tography }(5)+ \\
\text { microtiter plate (5) }\end{array}$ & N.D. [243] & \\
\hline GABA receptor & 2'-F-Py-RNA & filtration (11) & $\begin{array}{l}10.5,86 \mathrm{nM} \\
{[244]}\end{array}$ & \\
\hline $\begin{array}{l}\text { HER-3 ECD } \\
\text { oligomer }\end{array}$ & RNA & $\begin{array}{l}\text { filtration }(8)+ \\
\text { EMSA (7) }\end{array}$ & $\begin{array}{l}45 \mathrm{nM}(-\mathrm{hrg}) \\
400 \mathrm{nM}(+\mathrm{hrg}) \\
{[245]}\end{array}$ & \\
\hline$\alpha v \beta 3$ integrin & 2'-F-Py-RNA & $\begin{array}{l}\text { affinity polystyrene } \\
\text { beads }(7,15,17)\end{array}$ & $\begin{array}{l}2 \mathrm{nM}-4 \mu \mathrm{M} \\
{[246,247]}\end{array}$ & \\
\hline $\begin{array}{l}\text { KMP-11 } \\
\text { (leishmania) }\end{array}$ & DNA & $\begin{array}{l}\text { affinity colloidal } \\
\text { gold and } \\
\text { centrifugation (10) }\end{array}$ & N.D. [248] & \\
\hline MUC1-5TR & DNA & $\begin{array}{l}\text { affinity chroma- } \\
\text { tography }(10)\end{array}$ & 47-58 nM [249] & \\
\hline \multirow[t]{2}{*}{$\begin{array}{l}\mathrm{nAChR} \\
(\text { T.Californica })\end{array}$} & RNA & $\begin{array}{l}\text { filtration + EMSA } \\
\text { (9) }\end{array}$ & 2-12 nM [250] & \\
\hline & 2'-F-Py-RNA & $\begin{array}{l}\text { filtration + EMSA } \\
\text { (9) }\end{array}$ & $\begin{array}{l}0.47,4.1 \mathrm{nM} \\
{[251]}\end{array}$ & \\
\hline $\begin{array}{l}\text { neurotensin receptor } \\
\text { (NST-1, rat) }\end{array}$ & RNA & $\begin{array}{l}\text { affinity magnetic } \\
\text { beads ( } 7 \text { ) }\end{array}$ & $0.37-19 \mathrm{nM}$ [252] & \\
\hline PSMA & 2'-F-Py-RNA & $\begin{array}{l}\text { affinity magnetic } \\
\text { beads (6) }\end{array}$ & 2-12 nM [253] & \\
\hline $\begin{array}{l}\text { TNFR (RANK, } \\
\text { CD30, NGFR, }\end{array}$ & RNA & $\begin{array}{l}\text { affinity chroma- } \\
\text { tography (7) }\end{array}$ & $\begin{array}{l}0.11-960 \mathrm{nM} \\
{[254]}\end{array}$ & \\
\hline TRAIL-R2) & 2'-F-Py-RNA & & $0.1 \mu \mathrm{M}[254]$ & \\
\hline P-selectin & 2'-F-Py-RNA & $\begin{array}{l}\text { affinity chroma- } \\
\text { tography (12) }\end{array}$ & $\begin{array}{l}0.019-0.039 \mathrm{nM} \\
{[255]}\end{array}$ & \\
\hline \multirow[t]{2}{*}{ L-selectin } & DNA & $\begin{array}{l}\text { affinity chroma- } \\
\text { tography (17) }\end{array}$ & $1.8-5.5 \mathrm{nM}[256]$ & \\
\hline & $2^{\prime}-\mathrm{NH}_{2}-\mathrm{Py}-\mathrm{RNA}$ & $\begin{array}{l}\text { affinity chroma- } \\
\text { tography (14) }\end{array}$ & $3 \mathrm{nM}$ [257] & \\
\hline tenascin- $\mathrm{C}$ & $\begin{array}{l}\text { 2'-F-Py-2'- } \\
\text { OCH }-\mathrm{Pu}-\mathrm{RNA} \\
\text { DNA }\end{array}$ & $\begin{array}{l}\text { microtiter plates }(8, \\
11) \\
\text { cell monolayer }(21)\end{array}$ & $\begin{array}{l}5 \mathrm{nM}[258] \\
0.1-1 \mu \mathrm{M}[259]\end{array}$ & \\
\hline \multicolumn{5}{|l|}{ Enzymes } \\
\hline $\mathrm{APC}$ & RNA & filtration (7) & $0.12 \mu \mathrm{M}[260]$ & \\
\hline Factor VIIa & $2^{\prime}-\mathrm{NH}_{2}-\mathrm{Py}-\mathrm{RNA}$ & filtration (13) & $11-336 \mathrm{nM}[261]$ & \\
\hline
\end{tabular}


Table 2. Aptamer selected in vitro (continued)

\begin{tabular}{|c|c|c|c|c|}
\hline targets & types & $\begin{array}{l}\text { Separation } \\
\text { methods (rounds) }\end{array}$ & $\mathrm{Kds}$ & $\begin{array}{l}\text { PDB IDs } \\
\text { (3D-structures) }\end{array}$ \\
\hline Factor IXa & 2'-F-Py-RNA & filtration (8) & $0.58 \mathrm{nM}$ [45] & \\
\hline $\mathrm{hNE}$ & $\begin{array}{l}\text { valP-2'-NH }{ }_{2} \text {-Py- } \\
\text { RNA }\end{array}$ & EMSA (10) & $\begin{array}{l}71-170 \mathrm{nM} \\
{[71,262]}\end{array}$ & \\
\hline hnps-PLA2 & $2^{\prime}-\mathrm{NH}_{2}-\mathrm{Py}-\mathrm{RNA}$ & $\begin{array}{l}\text { affinity } \\
\text { chromatography + } \\
\text { filtration (11) }\end{array}$ & $1.7 \mathrm{nM}$ [263] & \\
\hline HCV NS3 & RNA & $\begin{array}{l}\text { filtration (6) } \\
\text { filtration (10) } \\
\text { filtration (9) } \\
\text { filtration (8) } \\
\text { affinity chroma- } \\
\text { tography (21) }\end{array}$ & $\begin{array}{l}0.2 \mu \mathrm{M}[264] \\
0.65 \mu \mathrm{M}[265] \\
10 \mathrm{nM}[266] \\
20 \mathrm{nM}[267] \\
0.99 \mathrm{nM}[268]\end{array}$ & \\
\hline lysozyme & RNA & filtration (12) & $31 \mathrm{nM}$ [130] & \\
\hline $\begin{array}{l}\text { protein kinase } \mathrm{C} \beta \\
\text { II }\end{array}$ & RNA & $\begin{array}{l}\text { filtration + EMSA } \\
\text { (14) }\end{array}$ & $7 \mathrm{nM}$ [269] & \\
\hline protein kinase $\mathrm{C} \delta$ & DNA & $\begin{array}{l}\text { capillary } \\
\text { electrophoresis } \\
(9)+\text { EMSA (1) }\end{array}$ & $122 \mathrm{nM}[270]$ & \\
\hline pro-urokinase & DNA & $\begin{array}{l}\text { affinity chroma- } \\
\text { tography (12) }\end{array}$ & N.D. [271] & \\
\hline ppERK2 and & RNA & filtration (15) & $1-5,50 \mathrm{nM}[5]$ & \\
\hline ERK2 & DNA & $\begin{array}{l}\text { filtration }(1)+ \\
\text { immunoblot }(1)\end{array}$ & $63 \mathrm{nM}$ [272] & \\
\hline PTPase (Yersinia) & RNA & filtration (8) & $18,28 \mathrm{nM}$ [273] & \\
\hline Raf-1 RBD & RNA & filtration (9) & $0.15-0.36 \mu \mathrm{M}[274]$ & \\
\hline subtilisin & RNA & $\begin{array}{l}\text { affinity chroma- } \\
\text { tography (8) }\end{array}$ & $2.5 \mu \mathrm{M}[275]$ & \\
\hline \multirow[t]{5}{*}{$\begin{array}{l}\alpha \text {-thrombin } \\
\text { (human) }\end{array}$} & DNA & $\begin{array}{l}\text { affinity chroma- } \\
\text { tography (5) }\end{array}$ & $25-200 \mathrm{nM}$ [276] & $\begin{array}{l}\text { 1HUT, 1HAP, } \\
1 \mathrm{HAO}[285] *\end{array}$ \\
\hline & & filtration (11) & $0.5 \mathrm{nM}$ [277] & 148D [286] \\
\hline & RNA & filtration (12) & $9-155$ nM [278] & 2IDN [287] \\
\hline & 4'-thio-RNA & filtration (10) & $4.7 \mathrm{nM}[36]$ & 1QDF, 1QDH \\
\hline & $\begin{array}{l}\text { 2'-deoxy-Pu-2'- } \\
\mathrm{OCH}_{3}-\mathrm{Py}\end{array}$ & $\begin{array}{l}\text { microtiter plate } \\
(6)\end{array}$ & 26-200 nM [31] & $\begin{array}{l}\text { [288] } \\
1 \mathrm{RDE} \text { [289] }\end{array}$ \\
\hline thrombin (bovine) & RNA & $\begin{array}{l}\text { microtiter plate } \\
\text { (7) }\end{array}$ & $0.16-0.24 \mu \mathrm{M}[279]$ & \\
\hline \multicolumn{5}{|l|}{$\begin{array}{l}\text { Growth factors } \\
\text { and cytokines }\end{array}$} \\
\hline angiopoietin-2 & 2'-F-Py-RNA & filtration (11) & $2.2 \mathrm{nM}[280]$ & \\
\hline bFGF & RNA & filtration $(10,13)$ & $0.1-23 \mathrm{nM}[281]$ & \\
\hline & $2^{\prime}-\mathrm{NH}_{2}-\mathrm{Py}-\mathrm{RNA}$ & filtration (11) & $0.35 \mathrm{nM}$ [282] & \\
\hline HGF & DNA & $? ?$ & $19-25 \mathrm{nM}$ [283] & \\
\hline
\end{tabular}


Table 2. Aptamer selected in vitro (continued)

\begin{tabular}{|c|c|c|c|c|}
\hline targets & types & $\begin{array}{l}\text { Separation } \\
\text { methods (rounds) }\end{array}$ & $\mathrm{Kds}$ & $\begin{array}{l}\text { PDB IDs } \\
\text { (3D-structures) }\end{array}$ \\
\hline hTSH & $2^{\prime}-\mathrm{NH}_{2}-\mathrm{Py}-\mathrm{RNA}$ & filtration (9) & $2.5-100 \mathrm{nM}[284]$ & \\
\hline IL-23 & $\begin{array}{l}\text { 2'-deoxy-Pu-2'- } \\
\mathrm{OCH}_{3}-\mathrm{Py}\end{array}$ & $\begin{array}{l}\text { microtiter plate } \\
(8)\end{array}$ & 8.4-30 nM [31] & \\
\hline interferon- $\gamma$ & $\begin{array}{l}\text { 2'-NH }{ }_{2} \text { and/or } \\
\text { 2'F-Py-RNA }\end{array}$ & filtration (17) & $1.8-760 \mathrm{nM}$ [29] & \\
\hline KGF & $\begin{array}{l}\text { 2'-NH }{ }_{2}-\mathrm{Py}-\mathrm{RNA} \\
\text { 2'F-Py-RNA }\end{array}$ & $\begin{array}{l}\text { filtration (8) } \\
\text { filtration (8) }\end{array}$ & $\begin{array}{l}400 \mathrm{pM}[28] \\
0.3-3 \mathrm{pM}[28]\end{array}$ & \\
\hline NGF & RNA & filtration (10) & $0.1-0.7 \mu \mathrm{M}[290]$ & \\
\hline oncostain $\mathrm{M}$ & $\begin{array}{l}\text { 2'-F-Py-2'- } \\
\mathrm{OCH}_{3}-\mathrm{Pu}-\mathrm{RNA}\end{array}$ & $\begin{array}{l}\text { microtiter plate } \\
\text { (12) }\end{array}$ & 7 nM [291] & \\
\hline PDGF & $\begin{array}{l}\text { DNA } \\
\text { DNA (PEG,3'T, } \\
\left.\text { 2'F, 2'- }-\mathrm{OCH}_{3}\right)\end{array}$ & $\operatorname{EMSA}(12)$ & $\begin{array}{l}0.1 \mathrm{nM}[292] \\
0.1 \mathrm{nM}[44]\end{array}$ & \\
\hline $\mathrm{TNF} \alpha$ & $2^{\prime}-\mathrm{NH}_{2}-\mathrm{Py}-\mathrm{RNA}$ & $? ?$ & ?? [293] & \\
\hline VEGF & RNA & filtration (13) & $0.19-20 \mathrm{nM}$ [294] & \\
\hline & $\begin{array}{l}\text { 2'- } \mathrm{NH}_{2}-\mathrm{Py}-2^{\prime}- \\
\mathrm{OCH}_{3}-\mathrm{Pu}-\mathrm{RNA} \\
\text { 2'--F-Py-2'- } \\
\mathrm{OCH}_{3}-\mathrm{Pu}-\mathrm{RNA} \\
\text { fully 2'-OCH } \\
\text { RNA }\end{array}$ & $\begin{array}{l}\text { filtration (11) } \\
\text { filtration (12) } \\
\text { microtiter plate } \\
(10)\end{array}$ & $\begin{array}{l}0.14-320 \mathrm{nM}[30] \\
0.049-0.13 \mathrm{nM} \\
{[295]} \\
1-4 \mathrm{nM}[26]\end{array}$ & \\
\hline \multicolumn{5}{|l|}{$\begin{array}{l}\text { Nucleic acids } \\
\text { binding proteins }\end{array}$} \\
\hline AMV RT & RNA & filtration (12) & $0.5-7.3 \mathrm{nM}[296]$ & \\
\hline B52 (drosophila) & RNA & filtration (9) & $20-50 \mathrm{nM}[11,297]$ & \\
\hline $\begin{array}{l}\text { colicin E3 }(E . \\
\text { coli) }\end{array}$ & RNA & filtration (12) & 2-14 nM [298] & \\
\hline $\begin{array}{l}\text { DNA polymerase } \\
\beta / \kappa(E . \text { coli })\end{array}$ & RNA & $\begin{array}{l}\text { filtration }+ \\
\text { microtiter plater } \\
\text { (7) }\end{array}$ & $0.3-0.5 \mu \mathrm{M}$ [299] & \\
\hline $\mathrm{E} 2 \mathrm{~F}$ & RNA & $\begin{array}{l}\text { affinity chroma- } \\
\text { tography (13) }\end{array}$ & $4 \mathrm{nM}$ [300] & \\
\hline $\begin{array}{l}\text { EF-Tu } \\
\text { (T. thermophilus) }\end{array}$ & RNA & filtration (6) & $\begin{array}{l}\text { 4.5-20 nM, 7-19 } \\
\mathrm{nM}[301]\end{array}$ & \\
\hline eIF4A (mouse) & RNA & $\begin{array}{l}\text { affinity } \\
\text { precipitation }(12, \\
12) \text { or filtration } \\
(15,12)\end{array}$ & $27 \mathrm{nM}-8 \mu \mathrm{M}$ [302] & $\begin{array}{l}\text { 1XWU, 1XWP } \\
{[310]}\end{array}$ \\
\hline eIF4E (mouse) & RNA & $\begin{array}{l}\text { affinity chroma- } \\
\text { tography (14) }\end{array}$ & $11.2 \mathrm{nM}$ [303] & \\
\hline eIF4G & RNA & $\begin{array}{l}\text { affinity chroma- } \\
\text { tography (12) }\end{array}$ & $0.1-0.3 \mu \mathrm{M}[304]$ & \\
\hline
\end{tabular}


Table 2. Aptamer selected in vitro (continued)

\begin{tabular}{|c|c|c|c|c|}
\hline targets & types & $\begin{array}{l}\text { Separation } \\
\text { methods (rounds) }\end{array}$ & Kds & $\begin{array}{l}\text { PDB IDs } \\
\text { (3D-structures) }\end{array}$ \\
\hline FIV RT & RNA & $\begin{array}{l}\text { filtration }(12)+ \\
\operatorname{EMSA}(8)\end{array}$ & 1.9-24 nM [305] & \\
\hline g5p (Ff phage) & DNA & $\operatorname{EMSA}(8)$ & $5 \mu \mathrm{M}[306]$ & \\
\hline \multirow[t]{2}{*}{ HCV RdRp (NS5B) } & DNA & $\begin{array}{l}\text { surface plasma } \\
\text { resonance }(10)\end{array}$ & $1.3-24 \mathrm{nM}$ [307] & \\
\hline & RNA & $\begin{array}{l}\text { affinity chroma- } \\
\text { tography (20) }\end{array}$ & N.D. [308] & \\
\hline HDM2 & RNA & $\begin{array}{l}\text { affinity chroma- } \\
\text { tography }(10)\end{array}$ & N.D. [309] & \\
\hline Hel-N1 & RNA & $\begin{array}{l}\text { immuno- } \\
\text { precipitation (3) }\end{array}$ & N.D. [312] & \\
\hline \multirow[t]{2}{*}{ HIV-1 integrase } & RNA & filtration (18) & $10-800 \mathrm{nM}$ [313] & \\
\hline & DNA & from [311] & $9-160 \mathrm{nM}$ [314] & 1Y8D [337] \\
\hline HIV-1 Tat protein & RNA & filtration $(9-11)$ & $0.12 \mathrm{nM}[315]$ & $1 \mathrm{NBK}[338]$ \\
\hline \multirow[t]{2}{*}{$\begin{array}{l}\text { HIV nucleocapsid } \\
\text { protein }\end{array}$} & RNA & $\begin{array}{l}\text { affinity chroma- } \\
\text { tography }(8-10)\end{array}$ & $0.49-2.4 \mathrm{nM}[316]$ & \\
\hline & & filtration (17) & $2 \mathrm{nM}$ [317] & \\
\hline $\begin{array}{l}\text { HIV-1 gag } \\
\text { polyprotein }\end{array}$ & RNA & filtration $(8,10)$ & $\begin{array}{l}1-50 \mathrm{nM} \\
\text { (nucleocapsid } \\
\text { proten); } \\
3-12 \mathrm{nM} \text { (matrix } \\
\text { protein) [318] }\end{array}$ & \\
\hline \multirow[t]{3}{*}{ HIV Rev } & RNA & filtration (4) & ?? [319] & 1ULL [339] \\
\hline & & filtration (6) & $1 \mathrm{nM}[320]$ & \\
\hline & & $\begin{array}{l}\text { affinity chroma- } \\
\text { tography (10) }\end{array}$ & $19-36 \mathrm{nM}[321]$ & 484D [340] \\
\hline \multirow[t]{2}{*}{ HIV RT } & RNA & filtration (9) & $5 \mathrm{nM}$ [322] & $1 \mathrm{HVU}[341] *$ \\
\hline & DNA & $\begin{array}{l}\text { filtration (15) } \\
\text { capillary } \\
\text { electrophoresis } \\
\text { (4) }\end{array}$ & $\begin{array}{l}1-11 \mathrm{nM}[323] \\
0.1-0.5 \mathrm{nM}[119]\end{array}$ & \\
\hline HIV RT (RNase H) & DNA & filtration (8) & $30 \mathrm{nM}$ [311] & \\
\hline HSV-1 US11 & RNA & $\begin{array}{l}\text { affinity magnetic } \\
\text { beads (12) }\end{array}$ & $70-220$ nM [324] & \\
\hline HutP & RNA & filtration (7) & 57-168 nM [325] & \\
\hline IHF (E. coli) & ds-DNA & EMSA (4) & $0.8-97 \mathrm{nM}[326]$ & \\
\hline $\mathrm{Ku}$ & RNA & $\begin{array}{l}\text { Filtration + } \\
\text { EMSA (7) }\end{array}$ & $0.3-4.5 \mathrm{nM}$ [327] & \\
\hline L22 (ribosomal) & RNA & $\begin{array}{l}\text { affinity chroma- } \\
\text { tography (9) }\end{array}$ & N.D. [328] & \\
\hline LXR (rat) & DNA & $\begin{array}{l}\text { affinity chroma- } \\
\text { tography (8) }\end{array}$ & $1.1-24$ pM [329] & \\
\hline \multirow[t]{2}{*}{ MetJ } & ds-DNA & EMSA $(11,12)$ & $\sim 2 \mathrm{nM}[330]$ & \\
\hline & RNA & No available & $0.2-0.4 \mathrm{nM}[331]$ & \\
\hline MMLV RT & RNA & filtration (17) & 7-23 nM [296] & \\
\hline
\end{tabular}


Table 2. Aptamer selected in vitro (continued)

\begin{tabular}{|c|c|c|c|c|}
\hline targets & types & $\begin{array}{l}\text { Separation } \\
\text { methods (rounds) }\end{array}$ & $\mathrm{Kds}$ & $\begin{array}{l}\text { PDB IDs } \\
\text { (3D-structures) }\end{array}$ \\
\hline $\begin{array}{l}\text { nucleolin (RBD12) } \\
\text { (hamster) }\end{array}$ & RNA & magnetic bead & 5-200 nM [332] & 1IE2 [342] \\
\hline NFATc & RNA & $\begin{array}{l}\text { pulldown (9) } \\
\text { affinity }\end{array}$ & $30 \mathrm{nM}[333]$ & \\
\hline $\mathrm{NF \kappa B}\left(\mathrm{p} 50_{2}\right)$ & RNA & $\begin{array}{l}\text { precipitation (10) } \\
\text { filtration (14) }\end{array}$ & $1.34 \mathrm{nM}[334]$ & $\begin{array}{l}\text { 1OOA [343] * } \\
2 \mathrm{JWV}[344]\end{array}$ \\
\hline$\left(\mathrm{p} 65_{2}\right)$ & & filtration (12) & $11-150 \mathrm{nM}$ [335] & \\
\hline rho $(E$. coli $)$ & RNA & filtration (8) & $0.1-10 \mathrm{nM}$ [336] & \\
\hline Rex & RNA & filtration (8) & $25-30 \mathrm{nM}[8]$ & 1EXY [345] \\
\hline RF-1 (E. coli) & RNA & $\begin{array}{l}\text { affinity chroma- } \\
\text { tography }+ \\
\text { filtration (11) }\end{array}$ & $30 \mathrm{nM}$ [347] & \\
\hline RNase H1 & DNA & filtration (9) & $10-80 \mathrm{nM}[348]$ & \\
\hline $\operatorname{RNAP}($ E. coli $)$ & DNA & $\begin{array}{l}\text { affinity chroma- } \\
\text { tography (11) }\end{array}$ & $0.1-6 \mathrm{nM}[349]$ & \\
\hline RNAP II (yeast) & RNA & filtration (15) & $20 \mathrm{nM}[350]$ & $2 \mathrm{~B} 63[366] *$ \\
\hline $\mathrm{S} 1$ protein $(E$. coli $)$ & RNA & filtration (13) & $4 \mathrm{nM}[351]$ & \\
\hline Sel B $(E$. coli $)$ & RNA & filtration (4) & $30 \mathrm{nM}$ [352] & \\
\hline T4 DNA polymerase & RNA & filtration (4) & $5-30 \mathrm{nM}[91]$ & \\
\hline $\begin{array}{l}\text { Taq DNA } \\
\text { polymerase }\end{array}$ & DNA & filtration (12) & 0.04-9 nM [346] & \\
\hline TBP (yeast) & RNA & $\begin{array}{l}\text { filtration+SPR+ } \\
\text { EMSA (12) } \\
\text { filtration (7) }\end{array}$ & $\begin{array}{l}\text { 3-10 nM [353] } \\
2 \mathrm{nM} \mathrm{[354]}\end{array}$ & \\
\hline \multirow[t]{2}{*}{ TCF-1 } & RNA & $\begin{array}{l}\text { GST-pull down } \\
\text { (13) }\end{array}$ & $0.1-0.5 \mu \mathrm{M}[355]$ & \\
\hline & & $\begin{array}{l}\text { affinity chroma- } \\
\text { tography (13) }\end{array}$ & $0.1 \mu \mathrm{M}[356]$ & \\
\hline $\begin{array}{l}\text { TF IIIA (xenopus } \\
\text { laevis) }\end{array}$ & RNA & EMSA (4) & $0.66-11.6 \mathrm{nM}$ [357] & \\
\hline Tra2 (drosophila) & RNA & filtration (10) & $1 \mu \mathrm{M}[358]$ & \\
\hline $\begin{array}{l}\text { tRNA }^{\text {Ile }} \text { synthetase } \\
\text { (E. coli) }\end{array}$ & DNA & $\begin{array}{l}\text { affinity chroma- } \\
\text { tography (7) }\end{array}$ & $1.5 \mu \mathrm{M}[359]$ & \\
\hline $\begin{array}{l}\text { tRNA }^{\text {Gln }} \text { synthetase } \\
\text { (E. coli) }\end{array}$ & tRNA variants & filtration (7) & $0.13 \mathrm{nM}[360]$ & 1EXD [360] \\
\hline TTF1 & DNA & $\begin{array}{l}\text { affinity magnetic } \\
\text { beads (15) }\end{array}$ & 3.3-67 nM [361] & \\
\hline Tth DNA & DNA & filtration (12) & $0.01-0.3 \mathrm{nM}[346]$ & \\
\hline polymerase & & $\begin{array}{l}\text { heterodivalent } \\
\text { aptamer from } \\
\text { [346] }\end{array}$ & $2 \mathrm{nM}[362]$ & \\
\hline U1A & RNA & $\begin{array}{l}\text { immuno- } \\
\text { precipitation (3) }\end{array}$ & $\sim \operatorname{lnM}-0.1 \mu \mathrm{M}$ & \\
\hline & & filtration (18) & 4.5-69 nM [130] & \\
\hline $\begin{array}{l}\text { Wilms tumor } \\
\text { suppressor (WT1) }\end{array}$ & RNA & $\begin{array}{l}\text { filtration }(10)+ \\
\operatorname{EMSA}(1)\end{array}$ & $0.7 \mu \mathrm{M}[364,365]$ & \\
\hline
\end{tabular}


Table 2. Aptamer selected in vitro (continued)

\begin{tabular}{|c|c|c|c|c|}
\hline targets & types & $\begin{array}{l}\text { Separation methods } \\
\text { (rounds) }\end{array}$ & $\mathrm{Kds}$ & $\begin{array}{l}\text { PDB IDs } \\
\text { (3D-structures) }\end{array}$ \\
\hline \multicolumn{5}{|l|}{ Prion } \\
\hline $\operatorname{rPrP}^{\mathrm{C}}$ (hamster) & RNA & $\begin{array}{l}\text { affinity chroma- } \\
\text { tography (11) }\end{array}$ & N.D.[367] & \\
\hline (human) & DNA & $\begin{array}{l}\text { affinity chroma- } \\
\text { tography (6) }\end{array}$ & $>10 \mathrm{nM}[368]$ & \\
\hline $\operatorname{PrP}^{\mathrm{SC}}$ fibril (hamster) & 2'-F-Py-RNA & centrifugation (7) & $26.9 \mathrm{nM}[369]$ & \\
\hline \multicolumn{5}{|l|}{ Toxins } \\
\hline abrin toxin & DNA & $\begin{array}{l}\text { affinity chroma- } \\
\text { tography (8) }\end{array}$ & 28-130 nM [370] & \\
\hline cholera whole toxin & DNA & $\begin{array}{l}\text { affinity magnetic } \\
\text { beads (5) }\end{array}$ & N.D. [371] & \\
\hline pepocin & RNA & filtration (8) & 20-30 nM [372] & \\
\hline \multirow[t]{4}{*}{ ricin toxin } & RNA & filtration (9) & $7.3 \mathrm{nM}[373]$ & \\
\hline & DNA & $\begin{array}{l}\text { affinity chroma- } \\
\text { tography (9) }\end{array}$ & $0.1-0.5 \mu \mathrm{M}[374]$ & \\
\hline & & capillary & $0.06-0.2 \mu \mathrm{M}$ & \\
\hline & & electrophoresis (4) & [374] & \\
\hline \multirow[t]{2}{*}{ SEB } & DNA & $\begin{array}{l}\text { affinity magnetic } \\
\text { beads (5) }\end{array}$ & N.D. [371] & \\
\hline & L-DNA & $\begin{array}{l}\text { affinity chroma- } \\
\text { tography (12) }\end{array}$ & $0.42 \mu \mathrm{M}[375]$ & \\
\hline \multicolumn{5}{|l|}{ Other proteins } \\
\hline alfalfa mosaic virus & RNA & filtration (6) & $0.3->6 \mu \mathrm{M}[376]$ & $1 \mathrm{XOK}[384] *$ \\
\hline coat protein & & filtration (7) & $0.2-0.5 \mu \mathrm{M}[377]$ & \\
\hline bacteriophage MS2 & RNA & filtration (12) & N.D. [378] & $1 \mathrm{U} 1 \mathrm{Y}[385] *$ \\
\hline and $Q \beta$ coat protein & & & & $\begin{array}{l}\text { 6MSF [386] } \\
5 \mathrm{MSF}, 7 \mathrm{MSF} \\
{[387] *}\end{array}$ \\
\hline $\begin{array}{l}\text { bacteriophage PP7 } \\
\text { coat protein }\end{array}$ & RNA & filtration $(4,12)$ & $0.7-25 \mathrm{nM}$ [379] & \\
\hline $\begin{array}{l}\text { bacteriaphage } \mathrm{R} 17 \\
\text { coat protein }\end{array}$ & RNA & filtration (11) & $5 \mathrm{nM}[380]$ & \\
\hline$\beta$-catenin & RNA & GST pull down (8) & $5 \mathrm{nM}[381]$ & \\
\hline CED-9 (C. elegans) & RNA & $\begin{array}{l}\text { filtration }(6)+ \\
\operatorname{EMSA}(3)\end{array}$ & 4, $16 \mathrm{nM}[7]$ & \\
\hline complement C5 & $\begin{array}{l}\text { 2'-F-Py-2'- } \\
\mathrm{OCH}_{3}-\mathrm{Pu}-\mathrm{RNA}\end{array}$ & filtration $(12,+8)$ & $2-5 \mathrm{nM}[382]$ & \\
\hline $\begin{array}{l}\text { cytochrome } \mathrm{C} \text { and } \\
\text { hemin }\end{array}$ & DNA & $\begin{array}{l}\text { affinity chroma- } \\
\text { tography (6) }\end{array}$ & $0.3-5 \mu \mathrm{M}[87]$ & \\
\hline $\begin{array}{l}\text { cytohesin } 1 \text { (sec } 7 \\
\text { domain) }\end{array}$ & RNA & $\begin{array}{l}\text { affinity chroma- } \\
\text { tography (6) }\end{array}$ & $5 \mathrm{nM}$ [383] & \\
\hline gp120 (HIV-1) & 2'-F-Py-RNA & $\begin{array}{l}\text { surface plasma } \\
\text { resonance (5) }\end{array}$ & $\begin{array}{l}5-20 \mathrm{nM}[388- \\
390]\end{array}$ & \\
\hline
\end{tabular}


Table 2. Aptamer selected in vitro (continued)

\begin{tabular}{|c|c|c|c|c|}
\hline targets & types & $\begin{array}{l}\text { Separation methods } \\
\text { (rounds) }\end{array}$ & Kds & $\begin{array}{l}\text { PDB IDs } \\
\text { (3D-structures) }\end{array}$ \\
\hline \multirow[t]{2}{*}{ hemagglutinin A } & RNA & $\begin{array}{l}\text { surface plasma } \\
\text { resonance }(5)\end{array}$ & $0.1 \mathrm{nM}$ [391] & \\
\hline & DNA & $\begin{array}{l}\text { Ni-NTA resin } \\
\text { affinity capture (3) }\end{array}$ & N.D. [392] & \\
\hline hemagglutinin B & RNA & filtration (9) & $45 \mathrm{nM}$ [393] & \\
\hline HSF (yeast) & RNA & $\begin{array}{l}\text { filtration + EMSA } \\
\text { (14) }\end{array}$ & 20-80 nM [394] & \\
\hline MCP-1 (mouse) & 2'-F-Py-RNA & $\begin{array}{l}\text { affinity magnetic } \\
\text { beads (6) }\end{array}$ & $\begin{array}{l}0.18-0.37 \mathrm{nM} \\
{[395]}\end{array}$ & \\
\hline \multirow[t]{3}{*}{ streptavidin } & 2'-F-Py-RNA & $\begin{array}{l}\text { affinity magnetic } \\
\text { beads (9) }\end{array}$ & $7 \mathrm{nM}$ [396] & \\
\hline & RNA & $\begin{array}{l}\text { affinity chroma- } \\
\text { tography }(6)+ \\
\text { EMSA (3) }\end{array}$ & 70-200 nM [397] & \\
\hline & DNA & $\begin{array}{l}\text { affinity magnetic } \\
\text { beads (13) }\end{array}$ & 57-85 nM [398] & \\
\hline Tax & RNA & filtration (6) & 70 nM [399] & \\
\hline $\begin{array}{l}\text { Wheat germ } \\
\text { agglutinin }\end{array}$ & 2'-F-Py-RNA & $\begin{array}{l}\text { affinity chroma- } \\
\text { tography (??) }\end{array}$ & $1-20 \mathrm{nM}[400]$ & \\
\hline \multicolumn{5}{|l|}{ Nucleic acids } \\
\hline 5S RNA (E. coli) & RNA & $\begin{array}{l}\text { affinity chroma- } \\
\text { tography (12) }\end{array}$ & $3 \mu \mathrm{M}$ [401] & \\
\hline 16S RNA A site & RNA & $\begin{array}{l}\text { affinity chroma- } \\
\text { tography (15) }\end{array}$ & $\begin{array}{l}1.24-2.9 \mu \mathrm{M} \\
{[402]}\end{array}$ & \\
\hline ds-DNA (16bp) & RNA & $\begin{array}{l}\text { affinity chroma- } \\
\text { tography (5) }\end{array}$ & N.D. [403] & \\
\hline ds-DNA (22bp) & RNA & $\begin{array}{l}\text { affinity chroma- } \\
\text { tography }(16,26)\end{array}$ & $0.18-63 \mu \mathrm{M}$ [404] & \\
\hline $\begin{array}{l}\text { hairpin (DNA, } \\
\text { RNA) }\end{array}$ & $\begin{array}{l}2^{\prime}-\mathrm{OCH}_{3}-\mathrm{Py}- \\
\text { RNA }\end{array}$ & $\begin{array}{l}\text { SacI digestion, no } \\
\text { separation (4) }\end{array}$ & N.D. $[405,406]$ & \\
\hline $\begin{array}{l}\text { HCV IRES domain } \\
\text { II }\end{array}$ & RNA & $\begin{array}{l}\text { affinity magnetic } \\
\text { beads (4) }\end{array}$ & $\begin{array}{l}28 \mathrm{nM}-33 \mu \mathrm{M} \\
{[407]}\end{array}$ & \\
\hline $\begin{array}{l}\text { HCV IRES domain } \\
\text { IIa }\end{array}$ & RNA & $\begin{array}{l}\text { streptavidin } \\
\text { magnetic bead } \\
\text { capture }(6)\end{array}$ & $35 \mathrm{nM}$ [408] & \\
\hline $\begin{array}{l}\text { HCV IRES domain } \\
\text { IIId }\end{array}$ & RNA & $\begin{array}{l}\text { affinity magnetic } \\
\text { beads (4) }\end{array}$ & 4.4-130 nM [409] & \\
\hline $\begin{array}{l}\text { HCV IRES domain } \\
\text { IV }\end{array}$ & RNA & $\begin{array}{l}\text { streptavidin } \\
\text { magnetic bead } \\
\text { capture (10) }\end{array}$ & $\begin{array}{l}70 \mathrm{nM}, 1 \mu \mathrm{M} \\
{[410]}\end{array}$ & \\
\hline $\begin{array}{l}\text { HCV SL1 (RNA } \\
\text { hairpin) }\end{array}$ & RNA & $\begin{array}{l}\text { streptavidin } \\
\text { magnetic bead } \\
\text { capture }(8)\end{array}$ & $20 \mathrm{nM}$ [410] & \\
\hline
\end{tabular}


Table 2. Aptamer selected in vitro (continued)

\begin{tabular}{|c|c|c|c|c|}
\hline targets & types & $\begin{array}{l}\text { Separation } \\
\text { methods (rounds) }\end{array}$ & Kds & $\begin{array}{l}\text { PD B IDs } \\
\text { (3D-structures) }\end{array}$ \\
\hline HIV DIS (RNA hairpin) & RNA & $\operatorname{EMSA}(5-7)$ & $1.3-6.8 \mathrm{nM}[411]$ & $\begin{array}{l}\text { 2B8R, 2B8S } \\
\text { [424] * } \\
1 \mathrm{BAU}[425]\end{array}$ \\
\hline \multirow[t]{6}{*}{ HIV TAR RNA element } & DNA & $\begin{array}{l}\text { streptavidin } \\
\text { magnetic bead } \\
\text { capture (15) }\end{array}$ & 20-120 nM [412] & \\
\hline & & $? ?$ & $50 \mathrm{nM}$ [413] & \\
\hline & & $\begin{array}{l}\text { streptavidin } \\
\text { magnetic bead } \\
\text { capture }(9,15)\end{array}$ & $\begin{array}{l}0.3-1 \mu \mathrm{M}, 20 \mathrm{nM} \\
{[414]}\end{array}$ & \\
\hline & & $\begin{array}{l}\text { affinity magnetic } \\
\text { beads (9) }\end{array}$ & $30-500 \mathrm{nM}[414]$ & \\
\hline & RNA & $\begin{array}{l}\text { streptavidin } \\
\text { magnetic bead } \\
\text { capture (9)+ } \\
\text { EMSA (1) }\end{array}$ & $30 \mathrm{nM}$ [415] & $\begin{array}{l}\text { 2OOM, 2PN9 } \\
{[426]}\end{array}$ \\
\hline & & $\begin{array}{l}\text { streptavidin } \\
\text { magnetic bead } \\
\text { capture }(8,9)\end{array}$ & $30-800 \mathrm{nM}$ [414] & \\
\hline tRNA $^{\text {Phe }}$ (yeast) & RNA & $\begin{array}{l}\text { affinity chroma- } \\
\text { tography (10) }\end{array}$ & $20 \mathrm{nM}[416]$ & \\
\hline $\begin{array}{l}\text { tyrS box antiterminator } \\
\text { (B. subtilis) }\end{array}$ & tRNA & $\begin{array}{l}\text { streptavidin } \\
\text { magnetic bead } \\
\text { capture }(7)\end{array}$ & $0.3,7 \mu \mathrm{M}[417]$ & \\
\hline \multicolumn{5}{|l|}{$\begin{array}{l}\text { RNAP, gag polyprotein, } \\
\text { nACHR on membrane } \\
\text { and U } 251 \text { glioblastoma } \\
\text { cells expressing } \\
\text { tenascin-C as shown in } \\
\text { Protein section }\end{array}$} \\
\hline $\begin{array}{l}\text { tartrate : synthetic } \\
\text { receptor complex }\end{array}$ & RNA & $\begin{array}{l}\text { affinity chroma- } \\
\text { tography (13) }\end{array}$ & $0.21 \mathrm{mM}[418]$ & \\
\hline anthrax spores & DNA & centrifuge (4) & $<10$ spores [419] & \\
\hline differentiated PC12 cells & DNA & centrifuge (6) & N.D. [420] & \\
\hline $\begin{array}{l}\text { PC12 MEN2A and } \\
\text { MEN2B cells expressing } \\
\text { RET }\end{array}$ & 2'F-Py-RNA & centrifuge (15) & 30-70 nM [421] & \\
\hline $\begin{array}{l}\text { human influenza A } \\
\text { virus/Panama/2007/1999 } \\
\text { (H3N2) (HA) }\end{array}$ & RNA & $\begin{array}{l}\text { filtration }(8)+ \\
\text { microtiter plate } \\
(2)\end{array}$ & $0.188 \mathrm{nM}[422]$ & \\
\hline Jurkat T cell leukemia & 2'F-Py-RNA & $\begin{array}{l}\text { centrifugation } \\
\text { (16) }\end{array}$ & N.D. [423] & \\
\hline
\end{tabular}


Table 2. Aptamer selected in vitro (continued)

\begin{tabular}{|c|c|c|c|c|}
\hline targets & types & $\begin{array}{l}\text { Separation } \\
\text { methods } \\
\text { (rounds) }\end{array}$ & Kds & $\begin{array}{l}\text { PD B IDs } \\
\text { (3D-structures) }\end{array}$ \\
\hline Leukemia cells & DNA & centrifugation & $0.8-227 \mathrm{nM}$ & \\
\hline CCRF-CEM & & (16) & [427] & \\
\hline M. tuberculosis & DNA & $\begin{array}{l}\text { centrifugation } \\
\text { (10) }\end{array}$ & $\begin{array}{l}0.4,5.3 \mu \mathrm{M} \\
{[428]}\end{array}$ & \\
\hline $\begin{array}{l}\text { Prohead (DNA- } \\
\text { gp3) }\end{array}$ & RNA & $\begin{array}{l}\text { sucrose gradient } \\
\text { centrifugation } \\
\text { (7) }\end{array}$ & N.D. [429] & \\
\hline $\mathrm{Q} \beta$ replicase & RNA & $\begin{array}{l}\text { filtration (12) } \\
\text { filtration (13) }\end{array}$ & $\begin{array}{l}1 \mathrm{nM} \mathrm{[430]} \\
10 \mathrm{nM}[430]\end{array}$ & \\
\hline $\begin{array}{l}\text { Ramos cells } \\
\text { (IGHM) }\end{array}$ & DNA & $\begin{array}{l}\text { centrifugation } \\
\text { (20) }\end{array}$ & $\begin{array}{l}0.76-207 \mathrm{nM} \\
{[431,432]}\end{array}$ & \\
\hline $\begin{array}{l}\text { Rouse sarcoma } \\
\text { virus }\end{array}$ & (2'F-Py)-RNA & filtration (12) & $<<0.1 \mu \mathrm{M}[433]$ & \\
\hline $\begin{array}{l}\text { Red blood cell } \\
\text { ghost (human } \\
\text { CD71) }\end{array}$ & DNA & filtration (25) & $1 \mathrm{nM}$ [434] & \\
\hline $30 \mathrm{~S}$ ribsome $\pm \mathrm{S} 1$ & RNA & filtration (11-13) & 4-7 nM [351] & \\
\hline ScVL-1 virus & RNA & EMSA (8) & $0.2-3 \mathrm{nM}$ [435] & \\
\hline $\begin{array}{l}\text { Trypanosome } \\
\text { cruzi }\end{array}$ & & & & \\
\hline $\begin{array}{l}\text { cell surface } \\
\text { receptor }\end{array}$ & 2'F-Py-RNA & $\begin{array}{l}\text { centrifugation } \\
\text { (8) }\end{array}$ & $\begin{array}{l}40-400 \mathrm{nM} \\
{[436]}\end{array}$ & \\
\hline $\begin{array}{l}\text { flagellar pocket } \\
\text { protein }\end{array}$ & RNA & $\begin{array}{l}\text { centrifugation } \\
\text { (12) }\end{array}$ & $60 \mathrm{nM}$ [437] & \\
\hline VSG protein & 2'F-Py-RNA & $\begin{array}{l}\text { VSG filtration }+ \\
\text { live parasite } \\
\text { centrifugation } \\
\text { (9) }\end{array}$ & $0.16-2$ nM [438] & \\
\hline $\begin{array}{l}\text { YPEN-1 cells (rat } \\
\text { pigpen) }\end{array}$ & DNA & $\begin{array}{l}\text { centrifugation } \\
\text { (8) }\end{array}$ & N.D. [439] & \\
\hline
\end{tabular}

PDB ID: protein data bank identity. If a PDB ID is followed by a *, it is a crystal structure. Otherwise the PDB ID is a solution structure determined by nuclear magnetic resonance. Matrix for affinity chromatography: agrose or sepharose unless indicated.

Matrix for filtration: nitrocellulose membrane

AChR: human acetylcholine receptor, AMV: avian myeloblastosis virus

APC: human activated protein C, ARM: arginine-rich motif, bFGF: basic fibroblast growth factor, $\alpha$-CGRP: rat neuropeptide calcitonin gene-related peptide, DIS: dimerization initiation site, eIF: eukaryotic initiation factors, EMSA: electrophoretic mobility shift assay

FMN: flavin mononucleotide, FAD: flavin adenine dinucleotide FIV: feline immunodeficiency virus, GnRH: Gonadotropin-releasing hormone HCV: hepatitis C virus, HGF: hepatocyte growth factor, HIV:huamn immunodeficiency virus hNE: Human neutrophil elastase, hnps-PLA2: human nonpancreatic secretory phospholipase A2 HSF: heat shock factor, HSV-1: Herpes simplex virus-1 
hTSH: human thyroid stimulating hormone

HutP: histidine utilizing protein, IGHM: Immunoglobin Heavy mu chain

IRES: internal ribosome entry site, IHF: integration host factor, Ig: immunoglobulin

KGF: keratinocyte growth factor, KMP-11: Kinetoplastid membrane protein-11

LXR: Liver X receptors, MCP-1: Monocyte chemoattractant protein-1

MMLV: moloney murine leukemia virus, mAb: monoclonal antibody

nAChR: nicotinic acetylcholine receptor, NAD: niacinamide adenine dinucleotide

NFATc: nuclear factor of activated T cells, NGF: nerve growth factor

NMM: N-mehtylmesoporphyrin IX, NMN: niacinamide mononucleotide

N/OFQ: Neuropeptide nociceptin/orphanin FQ

PDGF: platelet-derived growth factor, PSMA: prostate-specific membrane antigen

PTPase: Protein tyrosine phosphatase, RANK: receptor activator of NF-Kb,

RdRp: RNA-depedent RNA polymerase, RET: receptor tyrosine kinase,

RF-1: release factor 1, RNAP: RNA polymerase, RT: reverse transcriptase

SAM: S-adenosyl methionine SAH: S-adenosyl homocysteine

ScV: Saccharomyces cerevisiae double-stranded RNA virus, SEB: staphylococcal enterotoxin B

sLe ${ }^{\mathrm{x}}$ : sialyl Lewis X, SPR: surface plasma resonance

TBP: TATA-binding protein, TF: transcription factor

$\mathrm{T}^{\mathrm{HM}}$ : 5-N-(6-aminohexyl)carbamoylmethy-2'-deoxyuridine, TTF1: thyroid transcription factor 1

ValP: valyl phosphonate moiety, VEGF: vascular endothelial growth factor

VSG: variant surface glycoprotein 
Table 3. Allosteric aptamers

\begin{tabular}{|c|c|c|c|}
\hline effector & allosteric module & allosteric effect & reference \\
\hline hemin & cytc aptamer & Kd decreased by $15 x$ & {$[87]$} \\
\hline tetramethylrosamine & activation domain & activate transcription by $10 x$ & {$[83]$} \\
\hline $\begin{array}{l}\text { ATP, theophylline, or } \\
\text { flavine } \\
\text { mononucleotide }\end{array}$ & malachite green aptamer & Kd decrease by $3-25 x$ & {$[84]$} \\
\hline R06/DIS & DIS/R06 aptamer & Kd decreased by $10 x$ & [81] \\
\hline hybridization oligo & ATP aptamer & activate ATP aptamer & {$[88]$} \\
\hline hybridization oligo & $\begin{array}{l}\text { binary malachite green } \\
\text { aptamer }\end{array}$ & $\begin{array}{l}\text { activate malachite green } \\
\text { aptamer }\end{array}$ & {$[85]$} \\
\hline hydridization oligo & $\begin{array}{l}\text { binary Hoechst } 7 \mathrm{e} \\
\text { aptamer }\end{array}$ & activate Hoechst $7 \mathrm{e}$ aptamer & [86] \\
\hline glycine & glycine riboswitch & cooperativity & [89] \\
\hline
\end{tabular}




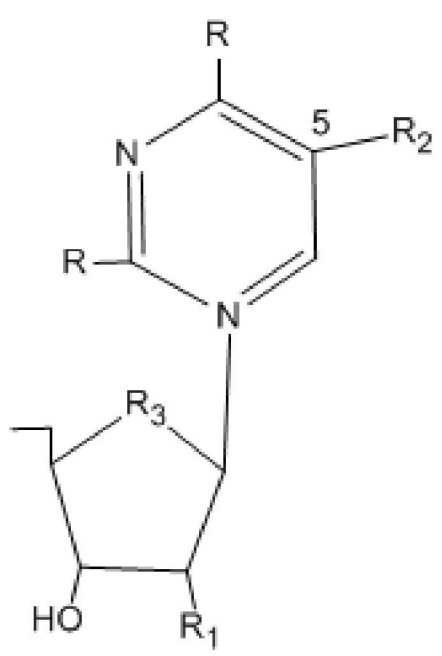

$\mathrm{C} / \mathrm{U} / \mathrm{T}$

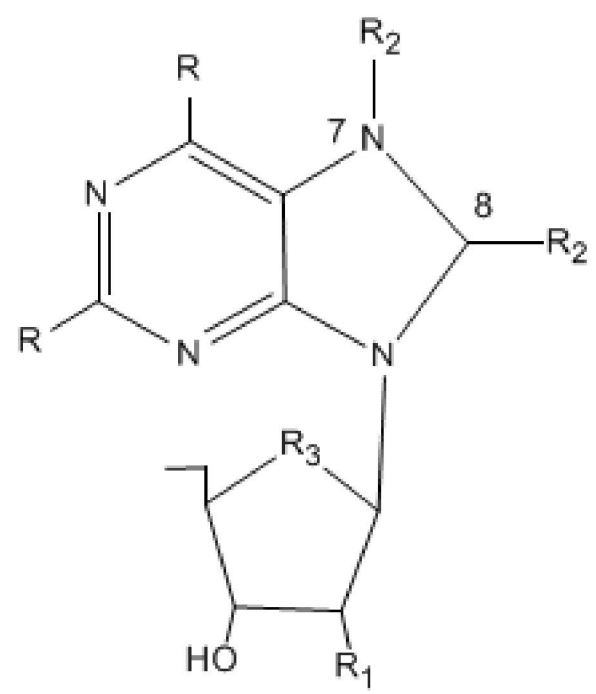

$\mathrm{A} / \mathrm{G}$

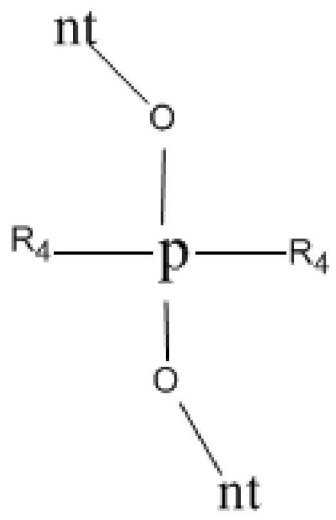

phosphodiester backbone

Figure 1. Modifications of nucleosides and phosphodiester backbone in aptamers $\mathrm{R} 1=\mathrm{H}, \mathrm{OH}, \mathrm{F}, \mathrm{OCH}_{3}$ and $\mathrm{NH}_{2} ; \mathrm{R} 2=$ hydrophobic, hydrophilic and charged groups $\mathrm{R} 3=\mathrm{O}, \mathrm{S} ; \mathrm{R} 4=\mathrm{O}, \mathrm{S}$ 
A

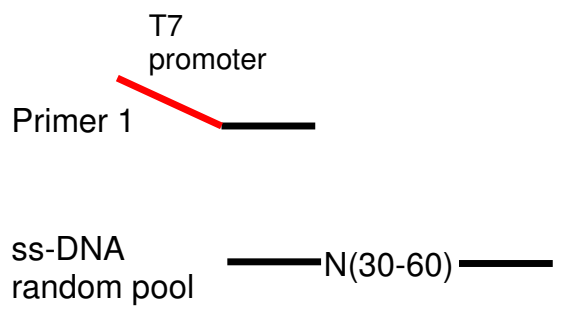

Primer 2
B

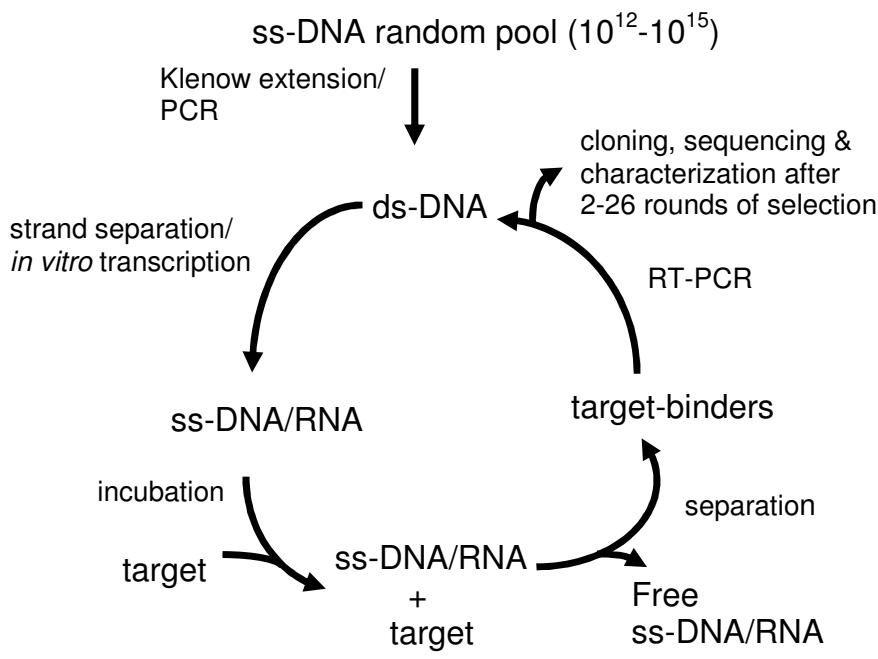

Figure 2. SELEX pool, primer and procedure

The SELEX pool contains a random region (30-60 nt long) flanked by two fixed regions. The fixed regions were used for PCR amplification with primer 1 and 2. A T7 promoter is required for in vitro transcription for RNA-SELEX but not for DNA-SELEX 


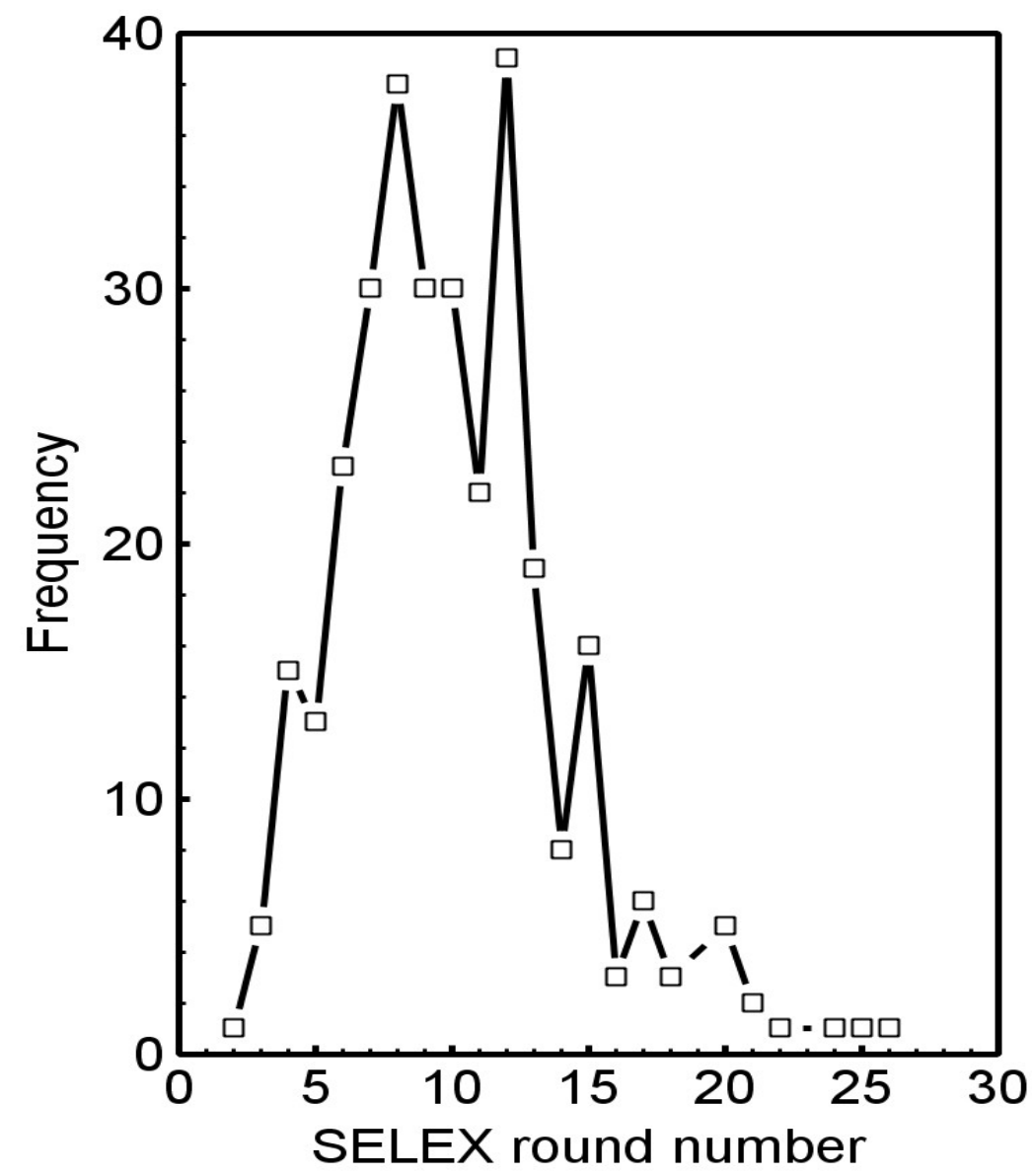

Figure 3. The distribution of SELEX rounds needed to obtain an aptamer summarized from Table 2 (total 312 SELEX experiments)

The average round number for a SELEX is 10 and the mode is 12 . 


\section{REFERENCES}

1. Klussmann, S., ed. The Aptamer Handbook. 2006, WILEY-VCH Verlag GmbH \& Co. KGaA: Weinheim.

2. Lee, J.F., J.R. Hesselberth, L.A. Meyers, and A.D. Ellington, Aptamer database. Nucleic Acids Res, 2004. 32:D95-100.

3. Huang, H.Y., C.H. Chien, K.H. Jen, and H.D. Huang, RegRNA: an integrated web server for identifying regulatory RNA motifs and elements. Nucleic Acids Res, 2006. 34:W429-34.

4. Jenison, R.D., S.C. Gill, A. Pardi, and B. Polisky, High-resolution molecular discrimination by RNA. Science, 1994. 263:1425-9.

5. Seiwert, S.D., T. Stines Nahreini, S. Aigner, N.G. Ahn, and O.C. Uhlenbeck, RNA aptamers as pathway-specific MAP kinase inhibitors. Chem Biol, 2000. 7:833-43.

6. Geiger, A., P. Burgstaller, H. von der Eltz, A. Roeder, and M. Famulok, RNA aptamers that bind L-arginine with sub-micromolar dissociation constants and high enantioselectivity. Nucleic Acids Res, 1996. 24:1029-36.

7. Yang, C., N. Yan, J. Parish, X. Wang, Y. Shi, and D. Xue, RNA aptamers targeting the cell death inhibitor CED-9 induce cell killing in Caenorhabditis elegans. J Biol Chem, 2006. 281:9137-44.

8. Baskerville, S., M. Zapp, and A.D. Ellington, Anti-Rex aptamers as mimics of the Rexbinding element. J Virol, 1999. 73:4962-71.

9. Blind, M., W. Kolanus, and M. Famulok, Cytoplasmic RNA modulators of an inside-out signal-transduction cascade. Proc Natl Acad Sci U S A, 1999. 96:3606-10.

10. Werstuck, G. and M.R. Green, Controlling gene expression in living cells through small molecule-RNA interactions. Science, 1998. 282:296-8.

11. Shi, H., B.E. Hoffman, and J.T. Lis, RNA aptamers as effective protein antagonists in a multicellular organism. Proc Natl Acad Sci U S A, 1999. 96:10033-8.

12. Drolet, D.W., J. Nelson, C.E. Tucker, P.M. Zack, K. Nixon, R. Bolin, M.B. Judkins, J.A. Farmer, J.L. Wolf, S.C. Gill, and R.A. Bendele, Pharmacokinetics and safety of an antivascular endothelial growth factor aptamer (NX1838) following injection into the vitreous humor of rhesus monkeys. Pharm Res, 2000. 17:1503-10.

13. Group, E.S., Anti-vascular endothelial growth factor therapy for subfoveal choroidal neovascularization secondary to age-related macular degeneration: phase II study results. Ophthalmology, 2003. 110:979-86.

14. Group, E.S., Preclinical and phase 1A clinical evaluation of an anti-VEGF pegylated aptamer (EYE001) for the treatment of exudative age-related macular degeneration. Retina, 2002. 22:143-52.

15. Wlotzka, B., S. Leva, B. Eschgfaller, J. Burmeister, F. Kleinjung, C. Kaduk, P. Muhn, H. Hess-Stumpp, and S. Klussmann, In vivo properties of an anti-GnRH Spiegelmer: an example of an oligonucleotide-based therapeutic substance class. Proc Natl Acad Sci U S A, 2002. 99:8898-902.

16. Presta, L.G., Engineering of therapeutic antibodies to minimize immunogenicity and optimize function. Adv Drug Deliv Rev, 2006. 58:640-56.

17. Henry, A.A. and F.E. Romesberg, The evolution of DNA polymerases with novel activities. Curr Opin Biotechnol, 2005. 16:370-7.

18. Holmberg, R.C., A.A. Henry, and F.E. Romesberg, Directed evolution of novel polymerases. Biomol Eng, 2005. 22:39-49.

19. Wilson, C. and A.D. Keefe, Building oligonucleotide therapeutics using non-natural chemistries. Curr Opin Chem Biol, 2006. 10:607-14. 
20. Kusser, W., Chemically modified nucleic acid aptamers for in vitro selections: evolving evolution. J Biotechnol, 2000. 74:27-38.

21. Eaton, B.E., The joys of in vitro selection: chemically dressing oligonucleotides to satiate protein targets. Curr Opin Chem Biol, 1997. 1:10-6.

22. Eaton, B.E., L. Gold, B.J. Hicke, N. Janjic, F.M. Jucker, D.P. Sebesta, T.M. Tarasow, M.C. Willis, and D.A. Zichi, Post-SELEX combinatorial optimization of aptamers. Bioorg Med Chem, 1997. 5:1087-96.

23. Ono, T., M. Scalf, and L.M. Smith, 2'-Fluoro modified nucleic acids: polymerase-directed synthesis, properties and stability to analysis by matrix-assisted laser desorption/ionization mass spectrometry. Nucleic Acids Res, 1997. 25:4581-8.

24. Padilla, R. and R. Sousa, Efficient synthesis of nucleic acids heavily modified with noncanonical ribose 2'-groups using a mutantT7 RNA polymerase (RNAP). Nucleic Acids Res, 1999. 27:1561-3.

25. Padilla, R. and R. Sousa, A Y639F/H784A T7 RNA polymerase double mutant displays superior properties for synthesizing RNAs with non-canonical NTPs. Nucleic Acids Res, 2002. 30:e138.

26. Burmeister, P.E., S.D. Lewis, R.F. Silva, J.R. Preiss, L.R. Horwitz, P.S. Pendergrast, T.G. McCauley, J.C. Kurz, D.M. Epstein, C. Wilson, and A.D. Keefe, Direct in vitro selection of a 2'-O-methyl aptamer to VEGF. Chem Biol, 2005. 12:25-33.

27. Chelliserrykattil, J. and A.D. Ellington, Evolution of a T7 RNA polymerase variant that transcribes 2'-O-methyl RNA. Nat Biotechnol, 2004. 22:1155-60.

28. Pagratis, N.C., C. Bell, Y.F. Chang, S. Jennings, T. Fitzwater, D. Jellinek, and C. Dang, Potent 2'-amino-, and 2'-fluoro-2'-deoxyribonucleotide RNA inhibitors of keratinocyte growth factor. Nat Biotechnol, 1997. 15:68-73.

29. Kubik, M.F., C. Bell, T. Fitzwater, S.R. Watson, and D.M. Tasset, Isolation and characterization of 2'-fluoro-, 2'-amino-, and 2'-fluoro-/amino-modified RNA ligands to human IFN-gamma that inhibit receptor binding. J Immunol, 1997. 159:259-67.

30. Green, L.S., D. Jellinek, C. Bell, L.A. Beebe, B.D. Feistner, S.C. Gill, F.M. Jucker, and N. Janjic, Nuclease-resistant nucleic acid ligands to vascular permeability factor/vascular endothelial growth factor. Chem Biol, 1995. 2:683-95.

31. Burmeister, P.E., C. Wang, J.R. Killough, S.D. Lewis, L.R. Horwitz, A. Ferguson, K.M. Thompson, P.S. Pendergrast, T.G. McCauley, M. Kurz, J. Diener, S.T. Cload, C. Wilson, and A.D. Keefe, 2'-Deoxy purine, 2'-O-methyl pyrimidine ( $\mathrm{dRmY})$ aptamers as candidate therapeutics. Oligonucleotides, 2006. 16:337-51.

32. Schmidt, K.S., S. Borkowski, J. Kurreck, A.W. Stephens, R. Bald, M. Hecht, M. Friebe, L. Dinkelborg, and V.A. Erdmann, Application of locked nucleic acids to improve aptamer in vivo stability and targeting function. Nucleic Acids Res, 2004. 32:5757-65.

33. Darfeuille, F., J.B. Hansen, H. Orum, C. Di Primo, and J.J. Toulme, LNA/DNA chimeric oligomers mimic RNA aptamers targeted to the TAR RNA element of HIV-1. Nucleic Acids Res, 2004. 32:3101-7.

34. Di Primo, C., I. Rudloff, S. Reigadas, A.A. Arzumanov, M.J. Gait, and J.J. Toulme, Systematic screening of LNA/2'-O-methyl chimeric derivatives of a TAR RNA aptamer. FEBS Lett, 2007. 581:771-4.

35. Hoshika, S., N. Minakawa, and A. Matsuda, Synthesis and physical and physiological properties of 4'-thioRNA: application to post-modification of RNA aptamer toward NFkappaB. Nucleic Acids Res, 2004. 32:3815-25.

36. Kato, Y., N. Minakawa, Y. Komatsu, H. Kamiya, N. Ogawa, H. Harashima, and A. Matsuda, New NTP analogs: the synthesis of 4'-thioUTP and 4'-thioCTP and their utility for SELEX. Nucleic Acids Res, 2005. 33:2942-51. 
37. Andreola, M.L., C. Calmels, J. Michel, J.J. Toulme, and S. Litvak, Towards the selection of phosphorothioate aptamers optimizing in vitro selection steps with phosphorothioate nucleotides. Eur J Biochem, 2000. 267:5032-40.

38. King, D.J., D.A. Ventura, A.R. Brasier, and D.G. Gorenstein, Novel combinatorial selection of phosphorothioate oligonucleotide aptamers. Biochemistry, 1998. 37:16489-93.

39. King, D.J., S.E. Bassett, X. Li, S.A. Fennewald, N.K. Herzog, B.A. Luxon, R. Shope, and D.G. Gorenstein, Combinatorial selection and binding of phosphorothioate aptamers targeting human NF-kappa B RelA(p65) and p50. Biochemistry, 2002. 41:9696-706.

40. Bassett, S.E., S.M. Fennewald, D.J. King, X. Li, N.K. Herzog, R. Shope, J.F. Aronson, B.A. Luxon, and D.G. Gorenstein, Combinatorial selection and edited combinatorial selection of

phosphorothioate aptamers targeting human nuclear factor-kappaB RelA/p50 and RelA/RelA. Biochemistry, 2004. 43:9105-15.

41. Somasunderam, A., M.R. Ferguson, D.R. Rojo, V. Thiviyanathan, X. Li, W.A. O'Brien, and D.G. Gorenstein, Combinatorial selection, inhibition, and antiviral activity of DNA thioaptamers targeting the RNase $\mathrm{H}$ domain of HIV-1 reverse transcriptase. Biochemistry, 2005. 44:10388-95.

42. Kang, J., M.S. Lee, S.J. Watowich, and D.G. Gorenstein, Combinatorial selection of a RNA thioaptamer that binds to Venezuelan equine encephalitis virus capsid protein. FEBS Lett, 2007. 581:2497-502.

43. Jhaveri, S., B. Olwin, and A.D. Ellington, In vitro selection of phosphorothiolated aptamers. Bioorg Med Chem Lett, 1998. 8:2285-90.

44. Floege, J., T. Ostendorf, U. Janssen, M. Burg, H.H. Radeke, C. Vargeese, S.C. Gill, L.S. Green, and N. Janjic, Novel approach to specific growth factor inhibition in vivo: antagonism of platelet-derived growth factor in glomerulonephritis by aptamers. Am J Pathol, 1999. 154:169-79.

45. Rusconi, C.P., E. Scardino, J. Layzer, G.A. Pitoc, T.L. Ortel, D. Monroe, and B.A. Sullenger, RNA aptamers as reversible antagonists of coagulation factor IXa. Nature, 2002. 419:90-4.

46. Dougan, H., D.M. Lyster, C.V. Vo, A. Stafford, J.I. Weitz, and J.B. Hobbs, Extending the lifetime of anticoagulant oligodeoxynucleotide aptamers in blood. Nucl Med Biol, 2000. 27:289-97.

47. Di Giusto, D.A. and G.C. King, Construction, stability, and activity of multivalent circular anticoagulant aptamers. J Biol Chem, 2004. 279:46483-9.

48. Di Giusto, D.A., S.M. Knox, Y. Lai, G.D. Tyrelle, M.T. Aung, and G.C. King, Multitasking by multivalent circular DNA aptamers. Chembiochem, 2006. 7:535-44.

49. Umekage, S. and Y. Kikuchi, Production of circular form of streptavidin RNA aptamer in vitro. Nucleic Acids Symp Ser (Oxf), 2006:323-4.

50. Umekage, S. and Y. Kikuchi, Production of circular streptavidin RNA aptamer in vivo. Nucleic Acids Symp Ser (Oxf), 2007:391-2.

51. Eulberg, D. and S. Klussmann, Spiegelmers: biostable aptamers. Chembiochem, 2003. 4:97983.

52. Watson, S.R., Y.F. Chang, D. O'Connell, L. Weigand, S. Ringquist, and D.H. Parma, Anti-Lselectin aptamers: binding characteristics, pharmacokinetic parameters, and activity against an intravascular target in vivo. Antisense Nucleic Acid Drug Dev, 2000. 10:63-75.

53. McCauley, T.G., J.C. Kurz, P.G. Merlino, S.D. Lewis, M. Gilbert, D.M. Epstein, and H.N. Marsh, Pharmacologic and pharmacokinetic assessment of anti-TGFbeta2 aptamers in rabbit plasma and aqueous humor. Pharm Res, 2006. 23:303-11.

54. Tucker, C.E., L.S. Chen, M.B. Judkins, J.A. Farmer, S.C. Gill, and D.W. Drolet, Detection and plasma pharmacokinetics of an anti-vascular endothelial growth factor oligonucleotideaptamer (NX1838) in rhesus monkeys. J Chromatogr B Biomed Sci Appl, 1999. 732:203-12. 
55. Willis, M.C., B.D. Collins, T. Zhang, L.S. Green, D.P. Sebesta, C. Bell, E. Kellogg, S.C. Gill, A. Magallanez, S. Knauer, R.A. Bendele, P.S. Gill, and N. Janjic, Liposome-anchored vascular endothelial growth factor aptamers. Bioconjug Chem, 1998. 9:573-82.

56. Healy, J.M., S.D. Lewis, M. Kurz, R.M. Boomer, K.M. Thompson, C. Wilson, and T.G. McCauley, Pharmacokinetics and biodistribution of novel aptamer compositions. Pharm Res, 2004. 21:2234-46.

57. Vaught, J.D., T. Dewey, and B.E. Eaton, T7 RNA polymerase transcription with 5-position modified UTP derivatives. J Am Chem Soc, 2004. 126:11231-7.

58. Jager, S., G. Rasched, H. Kornreich-Leshem, M. Engeser, O. Thum, and M. Famulok, A versatile toolbox for variable DNA functionalization at high density. J Am Chem Soc, 2005. 127:15071-82.

59. Kuwahara, M., J. Nagashima, M. Hasegawa, T. Tamura, R. Kitagata, K. Hanawa, S. Hososhima, T. Kasamatsu, H. Ozaki, and H. Sawai, Systematic characterization of 2'deoxynucleoside- 5'-triphosphate analogs as substrates for DNA polymerases by polymerase chain reaction and kinetic studies on enzymatic production of modified DNA. Nucleic Acids Res, 2006. 34:5383-94.

60. Kuwahara, M., K. Hanawa, K. Ohsawa, R. Kitagata, H. Ozaki, and H. Sawai, Direct PCR amplification of various modified DNAs having amino acids: convenient preparation of DNA libraries with high-potential activities for in vitro selection. Bioorg Med Chem, 2006. 14:2518-26.

61. Porter, K.W., J. Tomasz, F. Huang, A. Sood, and B.R. Shaw, N7-cyanoborane-2'deoxyguanosine 5'-triphosphate is a good substrate for DNA polymerase. Biochemistry, 1995. 34:11963-9.

62. Ghadessy, F.J., N. Ramsay, F. Boudsocq, D. Loakes, A. Brown, S. Iwai, A. Vaisman, R. Woodgate, and P. Holliger, Generic expansion of the substrate spectrum of a DNA polymerase by directed evolution. Nat Biotechnol, 2004. 22:755-9.

63. Latham, J.A., R. Johnson, and J.J. Toole, The application of a modified nucleotide in aptamer selection: novel thrombin aptamers containing 5-(1-pentynyl)-2'-deoxyuridine. Nucleic Acids Res, 1994. 22:2817-22.

64. Battersby, T.R., D.N. Ang, P. Burgstaller, S.C. Jurczyk, M.T. Bowser, D.D. Buchanan, R.T. Kennedy, and S.A. Benner, Quantitative analysis of receptors for adenosine nucleotides obtained via in vitro selection from a library incorporating a cationic nucleotide analog. $\mathrm{J} \mathrm{Am}$ Chem Soc, 1999. 121:9781-9.

65. Masud, M.M., M. Kuwahara, H. Ozaki, and H. Sawai, Sialyllactose-binding modified DNA aptamer bearing additional functionality by SELEX. Bioorg Med Chem, 2004. 12:1111-20.

66. Lato, S.M., N.D. Ozerova, K. He, Z. Sergueeva, B.R. Shaw, and D.H. Burke, Boroncontaining aptamers to ATP. Nucleic Acids Res, 2002. 30:1401-7.

67. Nutiu, R. and Y. Li, Aptamers with fluorescence-signaling properties. Methods, 2005. 37:1625.

68. Jensen, K.B., B.L. Atkinson, M.C. Willis, T.H. Koch, and L. Gold, Using in vitro selection to direct the covalent attachment of human immunodeficiency virus type 1 Rev protein to highaffinity RNA ligands. Proc Natl Acad Sci U S A, 1995. 92:12220-4.

69. Golden, M.C., B.D. Collins, M.C. Willis, and T.H. Koch, Diagnostic potential of PhotoSELEX-evolved ssDNA aptamers. J Biotechnol, 2000. 81:167-78.

70. Charlton, J., G.P. Kirschenheuter, and D. Smith, Highly potent irreversible inhibitors of neutrophil elastase generated by selection from a randomized DNA-valine phosphonate library. Biochemistry, 1997. 36:3018-26. 
71. Smith, D., G.P. Kirschenheuter, J. Charlton, D.M. Guidot, and J.E. Repine, In vitro selection of RNA-based irreversible inhibitors of human neutrophil elastase. Chem Biol, 1995. 2:74150 .

72. Lin, Y., A. Padmapriya, K.M. Morden, and S.D. Jayasena, Peptide conjugation to an in vitroselected DNA ligand improves enzyme inhibition. Proc Natl Acad Sci U S A, 1995. 92:11044-8.

73. Davis, K.A., B. Abrams, Y. Lin, and S.D. Jayasena, Use of a high affinity DNA ligand in flow cytometry. Nucleic Acids Res, 1996. 24:702-6.

74. Lucignani, G., Aptamers and in-beam PET for advanced diagnosis and therapy optimisation. Eur J Nucl Med Mol Imaging, 2006. 33:1095-7.

75. Santulli-Marotto, S., S.K. Nair, C. Rusconi, B. Sullenger, and E. Gilboa, Multivalent RNA aptamers that inhibit CTLA-4 and enhance tumor immunity. Cancer Res, 2003. 63:7483-9.

76. McNamara, J.O., D. Kolonias, F. Pastor, R.S. Mittler, L. Chen, P.H. Giangrande, B. Sullenger, and E. Gilboa, Multivalent 4-1BB binding aptamers costimulate CD8+ T cells and inhibit tumor growth in mice. J Clin Invest, 2008. 118:376-86.

77. Babendure, J.R., S.R. Adams, and R.Y. Tsien, Aptamers switch on fluorescence of triphenylmethane dyes. J Am Chem Soc, 2003. 125:14716-7.

78. Fusco, D., N. Accornero, B. Lavoie, S.M. Shenoy, J.M. Blanchard, R.H. Singer, and E. Bertrand, Single mRNA molecules demonstrate probabilistic movement in living mammalian cells. Curr Biol, 2003. 13:161-7.

79. Burke, D.H. and J.H. Willis, Recombination, RNA evolution, and bifunctional RNA molecules isolated through chimeric SELEX. Rna, 1998. 4:1165-75.

80. Elbaz, J., B. Shlyahovsky, D. Li, and I. Willner, Parallel analysis of two analytes in solutions or on surfaces by using a bifunctional aptamer: applications for biosensing and logic gate operations. Chembiochem, 2008. 9:232-9.

81. Boucard, D., J.J. Toulme, and C. Di Primo, Bimodal loop-loop interactions increase the affinity of RNA aptamers for HIV-1 RNA structures. Biochemistry, 2006. 45:1518-24.

82. Silverman, S.K., Rube Goldberg goes (ribo)nuclear? Molecular switches and sensors made from RNA. Rna, 2003. 9:377-83.

83. Buskirk, A.R., A. Landrigan, and D.R. Liu, Engineering a ligand-dependent RNA transcriptional activator. Chem Biol, 2004. 11:1157-63.

84. Stojanovic, M.N. and D.M. Kolpashchikov, Modular aptameric sensors. J Am Chem Soc, 2004. 126:9266-70.

85. Kolpashchikov, D.M., Binary malachite green aptamer for fluorescent detection of nucleic acids. J Am Chem Soc, 2005. 127:12442-3.

86. Sando, S., A. Narita, and Y. Aoyama, Light-Up Hoechst-DNA Aptamer Pair: Generation of an Aptamer-Selective Fluorophore from a Conventional DNA-Staining Dye. Chembiochem, 2007. 8:1795-803.

87. Chinnapen, D.J. and D. Sen, Hemin-stimulated docking of cytochrome c to a hemin-DNA aptamer complex. Biochemistry, 2002. 41:5202-12.

88. Cong, X. and M. Nilsen-Hamilton, Allosteric aptamers: targeted reversibly attenuated probes. Biochemistry, 2005. 44:7945-54.

89. Mandal, M., M. Lee, J.E. Barrick, Z. Weinberg, G.M. Emilsson, W.L. Ruzzo, and R.R. Breaker, A glycine-dependent riboswitch that uses cooperative binding to control gene expression. Science, 2004. 306:275-9.

90. Robertson, D.L. and G.F. Joyce, Selection in vitro of an RNA enzyme that specifically cleaves single-stranded DNA. Nature, 1990. 344:467-8.

91. Tuerk, C. and L. Gold, Systematic evolution of ligands by exponential enrichment: RNA ligands to bacteriophage T4 DNA polymerase. Science, 1990. 249:505-10. 
92. Ellington, A.D. and J.W. Szostak, In vitro selection of RNA molecules that bind specific ligands. Nature, 1990. 346:818-22.

93. Stoltenburg, R., C. Reinemann, and B. Strehlitz, SELEX--a (r)evolutionary method to generate high-affinity nucleic acid ligands. Biomol Eng, 2007. 24:381-403.

94. Jhaveri, S.D. and A.D. Ellington, In vitro selection of RNA aptamers to a protein target by filter immobilization. Curr Protoc Mol Biol, 2001. Chapter 24:Unit 243.

95. Pollard, J., S.D. Bell, and A.D. Ellington, Design, synthesis, and amplification of DNA pools for construction of combinatorial pools and libraries. Curr Protoc Mol Biol, 2001. Chapter 24:Unit 242.

96. Fitzwater, T. and B. Polisky, A SELEX primer. Methods Enzymol, 1996. 267:275-301.

97. Tuerk, C., Using the SELEX combinatorial chemistry process to find high affinity nucleic acid ligands to target molecules. Methods Mol Biol, 1997. 67:219-30.

98. Ciesiolka, J., M. Illangasekare, I. Majerfeld, T. Nickles, M. Welch, M. Yarus, and S. Zinnen, Affinity selection-amplification from randomized ribooligonucleotide pools. Methods Enzymol, 1996. 267:315-35.

99. Conrad, R.C., L. Giver, Y. Tian, and A.D. Ellington, In vitro selection of nucleic acid aptamers that bind proteins. Methods Enzymol, 1996. 267:336-67.

100. Gold, L., B. Polisky, O. Uhlenbeck, and M. Yarus, Diversity of oligonucleotide functions. Annu Rev Biochem, 1995. 64:763-97.

101. Sabeti, P.C., P.J. Unrau, and D.P. Bartel, Accessing rare activities from random RNA sequences: the importance of the length of molecules in the starting pool. Chem Biol, 1997. 4:767-74.

102. Gevertz, J., H.H. Gan, and T. Schlick, In vitro RNA random pools are not structurally diverse: a computational analysis. Rna, 2005. 11:853-63.

103. Legiewicz, M., C. Lozupone, R. Knight, and M. Yarus, Size, constant sequences, and optimal selection. Rna, 2005. 11:1701-9.

104. Kim, N., J.S. Shin, S. Elmetwaly, H.H. Gan, and T. Schlick, RagPools: RNA-As-GraphPools--a web server for assisting the design of structured RNA pools for in vitro selection. Bioinformatics, 2007. 23:2959-60.

105. Kim, N., H.H. Gan, and T. Schlick, A computational proposal for designing structured RNA pools for in vitro selection of RNAs. Rna, 2007. 13:478-92.

106. Majerfeld, I. and M. Yarus, An RNA pocket for an aliphatic hydrophobe. Nat Struct Biol, 1994. 1:287-92.

107. Kato, T., T. Takemura, K. Yano, K. Ikebukuro, and I. Karube, In vitro selection of DNA aptamers which bind to cholic acid. Biochim Biophys Acta, 2000. 1493:12-8.

108. Kato, T., K. Yano, K. Ikebukuro, and I. Karube, Interaction of three-way DNA junctions with steroids. Nucleic Acids Res, 2000. 28:1963-8.

109. Ellington, A.D., RNA selection. Aptamers achieve the desired recognition. Curr Biol, 1994. 4:427-9.

110. Irvine, D., C. Tuerk, and L. Gold, SELEXION. Systematic evolution of ligands by exponential enrichment with integrated optimization by non-linear analysis. J Mol Biol, 1991. 222:739-61.

111. Levine, H.A. and M. Nilsen-Hamilton, A mathematical analysis of SELEX. Comput Biol Chem, 2007. 31:11-35.

112. Clark, S.L. and V.T. Remcho, Electrochromatographic retention studies on a flavin-binding RNA aptamer sorbent. Anal Chem, 2003. 75:5692-6.

113. Gopinath, S.C., Methods developed for SELEX. Anal Bioanal Chem, 2007. 387:171-82. 
114. Drabovich, A., M. Berezovski, and S.N. Krylov, Selection of smart aptamers by equilibrium capillary electrophoresis of equilibrium mixtures (ECEEM). J Am Chem Soc, 2005. 127:11224-5.

115. Berezovski, M.V., M.U. Musheev, A.P. Drabovich, J.V. Jitkova, and S.N. Krylov, NonSELEX: selection of aptamers without intermediate amplification of candidate oligonucleotides. Nat Protoc, 2006. 1:1359-69.

116. Mendonsa, S.D. and M.T. Bowser, In vitro selection of high-affinity DNA ligands for human IgE using capillary electrophoresis. Anal Chem, 2004. 76:5387-92.

117. Mendonsa, S.D. and M.T. Bowser, In vitro evolution of functional DNA using capillary electrophoresis. J Am Chem Soc, 2004. 126:20-1.

118. Mendonsa, S.D. and M.T. Bowser, In vitro selection of aptamers with affinity for neuropeptide Y using capillary electrophoresis. J Am Chem Soc, 2005. 127:9382-3.

119. Mosing, R.K., S.D. Mendonsa, and M.T. Bowser, Capillary electrophoresis-SELEX selection of aptamers with affinity for HIV-1 reverse transcriptase. Anal Chem, 2005. 77:6107-12.

120. Berezovski, M., M. Musheev, A. Drabovich, and S.N. Krylov, Non-SELEX selection of aptamers. J Am Chem Soc, 2006. 128:1410-1.

121. Berezovski, M., A. Drabovich, S.M. Krylova, M. Musheev, V. Okhonin, A. Petrov, and S.N. Krylov, Nonequilibrium capillary electrophoresis of equilibrium mixtures: a universal tool for development of aptamers. J Am Chem Soc, 2005. 127:3165-71.

122. Musheev, M.U. and S.N. Krylov, Selection of aptamers by systematic evolution of ligands by exponential enrichment: addressing the polymerase chain reaction issue. Anal Chim Acta, 2006. 564:91-6.

123. Crameri, A. and W.P. Stemmer, 10(20)-fold aptamer library amplification without gel purification. Nucleic Acids Res, 1993. 21:4410.

124. Kang, J., M.S. Lee, and D.G. Gorenstein, The enhancement of PCR amplification of a random sequence DNA library by DMSO and betaine: application to in vitro combinatorial selection of aptamers. J Biochem Biophys Methods, 2005. 64:147-51.

125. Knight, R. and M. Yarus, Analyzing partially randomized nucleic acid pools: straight dope on doping. Nucleic Acids Res, 2003. 31:e30.

126. Bittker, J.A., B.V. Le, and D.R. Liu, Nucleic acid evolution and minimization by nonhomologous random recombination. Nat Biotechnol, 2002. 20:1024-9.

127. Cox, J.C. and A.D. Ellington, Automated selection of anti-protein aptamers. Bioorg Med Chem, 2001. 9:2525-31.

128. Cox, J.C., M. Rajendran, T. Riedel, E.A. Davidson, L.J. Sooter, T.S. Bayer, M. SchmitzBrown, and A.D. Ellington, Automated acquisition of aptamer sequences. Comb Chem High Throughput Screen, 2002. 5:289-99.

129. Eulberg, D., K. Buchner, C. Maasch, and S. Klussmann, Development of an automated in vitro selection protocol to obtain RNA-based aptamers: identification of a biostable substance P antagonist. Nucleic Acids Res, 2005. 33:e45.

130. Cox, J.C., A. Hayhurst, J. Hesselberth, T.S. Bayer, G. Georgiou, and A.D. Ellington, Automated selection of aptamers against protein targets translated in vitro: from gene to aptamer. Nucleic Acids Res, 2002. 30:e108.

131. Drolet, D.W., R.D. Jenison, D.E. Smith, D. Pratt, and B.J. Hicke, A high throughput platform for systematic evolution of ligands by exponential enrichment (SELEX). Comb Chem High Throughput Screen, 1999. 2:271-8.

132. Zhang, H., A. Hamasaki, E. Toshiro, Y. Aoyama, and Y. Ito, Automated in vitro selection to obtain functional oligonucleotides. Nucleic Acids Symp Ser, 2000:219-20.

133. Hybarger, G., J. Bynum, R.F. Williams, J.J. Valdes, and J.P. Chambers, A microfluidic SELEX prototype. Anal Bioanal Chem, 2006. 384:191-8. 
134. Ciesiolka, J., J. Gorski, and M. Yarus, Selection of an RNA domain that binds Zn2+. Rna, 1995. 1:538-50.

135. Hofmann, H.P., S. Limmer, V. Hornung, and M. Sprinzl, Ni2+-binding RNA motifs with an asymmetric purine-rich internal loop and a G-A base pair. Rna, 1997. 3:1289-300.

136. Sassanfar, M. and J.W. Szostak, An RNA motif that binds ATP. Nature, 1993. 364:550-3.

137. Burke, D.H. and L. Gold, RNA aptamers to the adenosine moiety of S-adenosyl methionine: structural inferences from variations on a theme and the reproducibility of SELEX. Nucleic Acids Res, 1997. 25:2020-4.

138. Gebhardt, K., A. Shokraei, E. Babaie, and B.H. Lindqvist, RNA aptamers to Sadenosylhomocysteine: kinetic properties, divalent cation dependency, and comparison with anti-S-adenosylhomocysteine antibody. Biochemistry, 2000. 39:7255-65.

139. Burgstaller, P. and M. Famulok, Isolation of RNA aptamer for biologival cofactors by in vitro selection. Angew. Chem. Int. Ed. Eng., 1994. 33:1084-7.

140. Burke, D.H. and D.C. Hoffman, A novel acidophilic RNA motif that recognizes coenzyme A. Biochemistry, 1998. 37:4653-63.

141. Saran, D., J. Frank, and D.H. Burke, The tyranny of adenosine recognition among RNA aptamers to coenzyme A. BMC Evol Biol, 2003. 3:26.

142. Koizumi, M. and R.R. Breaker, Molecular recognition of cAMP by an RNA aptamer. Biochemistry, 2000. 39:8983-92.

143. Meli, M., J. Vergne, J.L. Decout, and M.C. Maurel, Adenine-aptamer complexes: a bipartite RNA site that binds the adenine nucleic base. J Biol Chem, 2002. 277:2104-11.

144. Huizenga, D.E. and J.W. Szostak, A DNA aptamer that binds adenosine and ATP. Biochemistry, 1995. 34:656-65.

145. Klussmann, S., A. Nolte, R. Bald, V.A. Erdmann, and J.P. Furste, Mirror-image RNA that binds D-adenosine. Nat Biotechnol, 1996. 14:1112-5.

146. Wilson, C. and J.W. Szostak, In vitro evolution of a self-alkylating ribozyme. Nature, 1995. 374:777-82.

147. Wilson, C., J. Nix, and J. Szostak, Functional requirements for specific ligand recognition by a biotin-binding RNA pseudoknot. Biochemistry, 1998. 37:14410-9.

148. Lauhon, C.T. and J.W. Szostak, RNA aptamers that bind flavin and nicotinamide redox cofactors. J Am Chem Soc, 1995. 117:1246-57.

149. Roychowdhury-Saha, M., S.M. Lato, E.D. Shank, and D.H. Burke, Flavin recognition by an RNA aptamer targeted toward FAD. Biochemistry, 2002. 41:2492-9.

150. Davis, J.H. and J.W. Szostak, Isolation of high-affinity GTP aptamers from partially structured RNA libraries. Proc Natl Acad Sci U S A, 2002. 99:11616-21.

151. Haller, A.A. and P. Sarnow, In vitro selection of a 7-methyl-guanosine binding RNA that inhibits translation of capped mRNA molecules. Proc Natl Acad Sci U S A, 1997. 94:8521-6.

152. Lorsch, J.R. and J.W. Szostak, In vitro selection of RNA aptamers specific for cyanocobalamin. Biochemistry, 1994. 33:973-82.

153. Kiga, D., Y. Futamura, K. Sakamoto, and S. Yokoyama, An RNA aptamer to the xanthine/guanine base with a distinctive mode of purine recognition. Nucleic Acids Res, 1998. 26:1755-60.

154. Famulok, M. and J. Szostak, Stereospecific Recognition of Tryptophan Agarose by in Vitro Selected RNA. J. Am. Chem. SOC., 1992. 114:3990-1.

155. Connell, G.J., M. Illangesekare, and M. Yarus, Three small ribooligonucleotides with specific arginine sites. Biochemistry, 1993. 32:5497-502.

156. Famulok, M., Molecular Recognition of Amino Acids by RNA- Apt amers: An L-Citrulline Binding RNA Motif and Its Evolution into an L-Arginine Binder. J. Am. Chem. SOC., 1994. 116:1698-706. 
157. Tao, J. and A.D. Frankel, Arginine-binding RNAs resembling TAR identified by in vitro selection. Biochemistry, 1996. 35:2229-38.

158. Dieckmann, T., E. Suzuki, G.K. Nakamura, and J. Feigon, Solution structure of an ATPbinding RNA aptamer reveals a novel fold. Rna, 1996. 2:628-40.

159. Jiang, F., R. Fiala, D. Live, R.A. Kumar, and D.J. Patel, RNA folding topology and intermolecular contacts in the AMP-RNA aptamer complex. Biochemistry, 1996. 35:1325066.

160. Lin, C.H. and D.J. Patel, Structural basis of DNA folding and recognition in an AMP-DNA aptamer complex: distinct architectures but common recognition motifs for DNA and RNA aptamers complexed to AMP. Chem Biol, 1997. 4:817-32.

161. Nix, J., D. Sussman, and C. Wilson, The 1.3 A crystal structure of a biotin-binding pseudoknot and the basis for RNA molecular recognition. J Mol Biol, 2000. 296:1235-44.

162. Fan, P., A.K. Suri, R. Fiala, D. Live, and D.J. Patel, Molecular recognition in the FMN-RNA aptamer complex. J Mol Biol, 1996. 258:480-500.

163. Carothers, J.M., J.H. Davis, J.J. Chou, and J.W. Szostak, Solution structure of an informationally complex high-affinity RNA aptamer to GTP. Rna, 2006. 12:567-79.

164. Sussman, D., J.C. Nix, and C. Wilson, The structural basis for molecular recognition by the vitamin B 12 RNA aptamer. Nat Struct Biol, 2000. 7:53-7.

165. Sussman, D. and C. Wilson, A water channel in the core of the vitamin B(12) RNA aptamer. Structure, 2000. 8:719-27.

166. Yang, Y., M. Kochoyan, P. Burgstaller, E. Westhof, and M. Famulok, Structural basis of ligand discrimination by two related RNA aptamers resolved by NMR spectroscopy. Science, 1996. 272:1343-7.

167. Nolte, A., S. Klussmann, R. Bald, V.A. Erdmann, and J.P. Furste, Mirror-design of Loligonucleotide ligands binding to L-arginine. Nat Biotechnol, 1996. 14:1116-9.

168. Harada, K. and A.D. Frankel, Identification of two novel arginine binding DNAs. Embo J, 1995. 14:5798-811.

169. Majerfeld, I., D. Puthenvedu, and M. Yarus, RNA affinity for molecular L-histidine; genetic code origins. J Mol Evol, 2005. 61:226-35.

170. Majerfeld, I. and M. Yarus, Isoleucine:RNA sites with associated coding sequences. Rna, 1998. 4:471-8.

171. Mannironi, C., C. Scerch, P. Fruscoloni, and G.P. Tocchini-Valentini, Molecular recognition of amino acids by RNA aptamers: the evolution into an L-tyrosine binder of a dopaminebinding RNA motif. Rna, 2000. 6:520-7.

172. Vianini, E., M. Palumbo, and B. Gatto, In vitro selection of DNA aptamers that bind Ltyrosinamide. Bioorg Med Chem, 2001. 9:2543-8.

173. Burke, D.H., D.C. Hoffman, A. Brown, M. Hansen, A. Pardi, and L. Gold, RNA aptamers to the peptidyl transferase inhibitor chloramphenicol. Chem Biol, 1997. 4:833-43.

174. Kwon, M., S.M. Chun, S. Jeong, and J. Yu, In vitro selection of RNA against kanamycin B. Mol Cells, 2001. 11:303-11.

175. Lato, S.M., A.R. Boles, and A.D. Ellington, In vitro selection of RNA lectins: using combinatorial chemistry to interpret ribozyme evolution. Chem Biol, 1995. 2:291-303.

176. Schurer, H., K. Stembera, D. Knoll, G. Mayer, M. Blind, H.H. Forster, M. Famulok, P. Welzel, and U. Hahn, Aptamers that bind to the antibiotic moenomycin A. Bioorg Med Chem, 2001. 9:2557-63.

177. Wallis, M.G., U. von Ahsen, R. Schroeder, and M. Famulok, A novel RNA motif for neomycin recognition. Chem Biol, 1995. 2:543-52.

178. Wallace, S.T. and R. Schroeder, In vitro selection and characterization of streptomycinbinding RNAs: recognition discrimination between antibiotics. Rna, 1998. 4:112-23. 
179. Berens, C., A. Thain, and R. Schroeder, A tetracycline-binding RNA aptamer. Bioorg Med Chem, 2001. 9:2549-56.

180. Muller, M., J.E. Weigand, O. Weichenrieder, and B. Suess, Thermodynamic characterization of an engineered tetracycline-binding riboswitch. Nucleic Acids Res, 2006. 34:2607-17.

181. Wang, Y. and R.R. Rando, Specific binding of aminoglycoside antibiotics to RNA. Chem Biol, 1995. 2:281-90.

182. Wallis, M.G., B. Streicher, H. Wank, U. von Ahsen, E. Clodi, S.T. Wallace, M. Famulok, and R. Schroeder, In vitro selection of a viomycin-binding RNA pseudoknot. Chem Biol, 1997. 4:357-66.

183. Holeman, L.A., S.L. Robinson, J.W. Szostak, and C. Wilson, Isolation and characterization of fluorophore-binding RNA aptamers. Fold Des, 1998. 3:423-31.

184. Lin, C.H. and D.J. Patel, Encapsulating an amino acid in a DNA fold. Nat Struct Biol, 1996. 3:1046-50.

185. Lin, C.H., W. Wang, R.A. Jones, and D.J. Patel, Formation of an amino-acid-binding pocket through adaptive zippering-up of a large DNA hairpin loop. Chem Biol, 1998. 5:555-72.

186. Robertson, S.A., K. Harada, A.D. Frankel, and D.E. Wemmer, Structure determination and binding kinetics of a DNA aptamer-argininamide complex. Biochemistry, 2000. 39:946-54.

187. Jiang, L., A. Majumdar, W. Hu, T.J. Jaishree, W. Xu, and D.J. Patel, Saccharide-RNA recognition in a complex formed between neomycin B and an RNA aptamer. Structure, 1999. 7:817-27.

188. Tereshko, V., E. Skripkin, and D.J. Patel, Encapsulating streptomycin within a small 40-mer RNA. Chem Biol, 2003. 10:175-87.

189. Jiang, L., A.K. Suri, R. Fiala, and D.J. Patel, Saccharide-RNA recognition in an aminoglycoside antibiotic-RNA aptamer complex. Chem Biol, 1997. 4:35-50.

190. Jiang, L. and D.J. Patel, Solution structure of the tobramycin-RNA aptamer complex. Nat Struct Biol, 1998. 5:769-74.

191. Grate, D. and C. Wilson, Laser-mediated, site-specific inactivation of RNA transcripts. Proc Natl Acad Sci U S A, 1999. 96:6131-6.

192. Ellington, A.D. and J.W. Szostak, Selection in vitro of single-stranded DNA molecules that fold into specific ligand-binding structures. Nature, 1992. 355:850-2.

193. Wilson, C. and J.W. Szostak, Isolation of a fluorophore-specific DNA aptamer with weak redox activity. Chem Biol, 1998. 5:609-17.

194. Welch, M., I. Majerfeld, and M. Yarus, 23S rRNA similarity from selection for peptidyl transferase mimicry. Biochemistry, 1997. 36:6614-23.

195. Stojanovic, M.N., P. de Prada, and D.W. Landry, Fluorescent Sensors Based on Aptamer Self-Assembly. J. Am. Chem. Soc., 2000. 122:11547-8.

196. Stojanovic, M.N., P. de Prada, and D.W. Landry, Aptamer-based folding fluorescent sensor for cocaine. J Am Chem Soc, 2001. 123:4928-31.

197. Win, M.N., J.S. Klein, and C.D. Smolke, Codeine-binding RNA aptamers and rapid determination of their binding constants using a direct coupling surface plasmon resonance assay. Nucleic Acids Res, 2006. 34:5670-82.

198. Wochner, A., M. Menger, D. Orgel, B. Cech, M. Rimmele, V.A. Erdmann, and J. Glokler, A DNA aptamer with high affinity and specificity for therapeutic anthracyclines. Anal Biochem, 2007.

199. Mannironi, C., A. Di Nardo, P. Fruscoloni, and G.P. Tocchini-Valentini, In vitro selection of dopamine RNA ligands. Biochemistry, 1997. 36:9726-34.

200. Mann, D., C. Reinemann, R. Stoltenburg, and B. Strehlitz, In vitro selection of DNA aptamers binding ethanolamine. Biochem Biophys Res Commun, 2005. 338:1928-34. 
201. Okazawa, A., H. Maeda, E. Fukusaki, Y. Katakura, and A. Kobayashi, In vitro selection of hematoporphyrin binding DNA aptamers. Bioorg Med Chem Lett, 2000. 10:2653-6.

202. Brockstedt, U., A. Uzarowska, A. Montpetit, W. Pfau, and D. Labuda, In vitro evolution of RNA aptamers recognizing carcinogenic aromatic amines. Biochem Biophys Res Commun, 2004. 313:1004-8.

203. Li, Y., C.R. Geyer, and D. Sen, Recognition of anionic porphyrins by DNA aptamers. Biochemistry, 1996. 35:6911-22.

204. Li, Y. and D. Sen, A catalytic DNA for porphyrin metallation. Nat Struct Biol, 1996. 3:743-7.

205. Travascio, P., Y. Li, and D. Sen, DNA-enhanced peroxidase activity of a DNA-aptamerhemin complex. Chem Biol, 1998. 5:505-17.

206. Levesque, D., J.D. Beaudoin, S. Roy, and J.P. Perreault, In vitro selection and characterization of RNA aptamers binding thyroxine hormone. Biochem J, 2007. 403:129-38.

207. Baugh, C., D. Grate, and C. Wilson, 2.8 A crystal structure of the malachite green aptamer. J Mol Biol, 2000. 301:117-28.

208. Flinders, J., S.C. DeFina, D.M. Brackett, C. Baugh, C. Wilson, and T. Dieckmann, Recognition of planar and nonplanar ligands in the malachite green-RNA aptamer complex. Chembiochem, 2004. 5:62-72.

209. Zimmermann, G.R., R.D. Jenison, C.L. Wick, J.P. Simorre, and A. Pardi, Interlocking structural motifs mediate molecular discrimination by a theophylline-binding RNA. Nat Struct Biol, 1997. 4:644-9.

210. Clore, G.M. and J. Kuszewski, Improving the accuracy of NMR structures of RNA by means of conformational database potentials of mean force as assessed by complete dipolar coupling cross-validation. J Am Chem Soc, 2003. 125:1518-25.

211. Yang, Q., I.J. Goldstein, H.Y. Mei, and D.R. Engelke, DNA ligands that bind tightly and selectively to cellobiose. Proc Natl Acad Sci U S A, 1998. 95:5462-7.

212. Fukusaki, E., T. Kato, H. Maeda, N. Kawazoe, Y. Ito, A. Okazawa, S. Kajiyama, and A. Kobayashi, DNA aptamers that bind to chitin. Bioorg Med Chem Lett, 2000. 10:423-5.

213. Jeong, S., T. Eom, S. Kim, S. Lee, and J. Yu, In vitro selection of the RNA aptamer against the Sialyl Lewis $\mathrm{X}$ and its inhibition of the cell adhesion. Biochem Biophys Res Commun, 2001. 281:237-43.

214. Srisawat, C., I.J. Goldstein, and D.R. Engelke, Sephadex-binding RNA ligands: rapid affinity purification of RNA from complex RNA mixtures. Nucleic Acids Res, 2001. 29:E4.

215. Ylera, F., R. Lurz, V.A. Erdmann, and J.P. Furste, Selection of RNA aptamers to the Alzheimer's disease amyloid peptide. Biochem Biophys Res Commun, 2002. 290:1583-8.

216. Fukusho, S., H. Furusawa, and Y. Okahata, In vitro selection and analysis of RNA aptamer recognize arginine-rich motif (ARM) model peptide on a QCM. Nucleic Acids Symp Ser, 2000:187-8.

217. Vater, A., F. Jarosch, K. Buchner, and S. Klussmann, Short bioactive Spiegelmers to migraine-associated calcitonin gene-related peptide rapidly identified by a novel approach: tailored-SELEX. Nucleic Acids Res, 2003. 31:e130.

218. Gilbert, B.A., M. Sha, S.T. Wathen, and R.R. Rando, RNA aptamers that specifically bind to a K Ras-derived farnesylated peptide. Bioorg Med Chem, 1997. 5:1115-22.

219. Helmling, S., C. Maasch, D. Eulberg, K. Buchner, W. Schroder, C. Lange, S. Vonhoff, B. Wlotzka, M.H. Tschop, S. Rosewicz, and S. Klussmann, Inhibition of ghrelin action in vitro and in vivo by an RNA-Spiegelmer. Proc Natl Acad Sci U S A, 2004. 101:13174-9.

220. Jarosch, F., K. Buchner, and S. Klussmann, In vitro selection using a dual RNA library that allows primerless selection. Nucleic Acids Res, 2006. 34:e86. 
221. Leva, S., A. Lichte, J. Burmeister, P. Muhn, B. Jahnke, D. Fesser, J. Erfurth, P. Burgstaller, and S. Klussmann, GnRH binding RNA and DNA Spiegelmers: a novel approach toward GnRH antagonism. Chem Biol, 2002. 9:351-9.

222. Ferreira, C.S., C.S. Matthews, and S. Missailidis, DNA aptamers that bind to MUC1 tumour marker: design and characterization of MUC1-binding single-stranded DNA aptamers. Tumour Biol, 2006. 27:289-301.

223. Faulhammer, D., B. Eschgfaller, S. Stark, P. Burgstaller, W. Englberger, J. Erfurth, F. Kleinjung, J. Rupp, S. Dan Vulcu, W. Schroder, S. Vonhoff, H. Nawrath, C. Gillen, and S. Klussmann, Biostable aptamers with antagonistic properties to the neuropeptide nociceptin/orphanin FQ. Rna, 2004. 10:516-27.

224. Proske, D., M. Hofliger, R.M. Soll, A.G. Beck-Sickinger, and M. Famulok, A Y2 receptor mimetic aptamer directed against neuropeptide Y. J Biol Chem, 2002. 277:11416-22.

225. Proske, D., S. Gilch, F. Wopfner, H.M. Schatzl, E.L. Winnacker, and M. Famulok, Prionprotein-specific aptamer reduces PrPSc formation. Chembiochem, 2002. 3:717-25.

226. Nieuwlandt, D., M. Wecker, and L. Gold, In vitro selection of RNA ligands to substance P. Biochemistry, 1995. 34:5651-9.

227. Williams, K.P., X.H. Liu, T.N. Schumacher, H.Y. Lin, D.A. Ausiello, P.S. Kim, and D.P. Bartel, Bioactive and nuclease-resistant L-DNA ligand of vasopressin. Proc Natl Acad Sci U S A, 1997. 94:11285-90.

228. Gu, K.D. and M. Famulok, [In vitro selection of specific aptamers against microcystin-LR.]. Zhonghua Yu Fang Yi Xue Za Zhi, 2004. 38:369-73.

229. Gening, L.V., S.A. Klincheva, A.S. Gusev, A.Y. Surovoy, and V.K. Potapov, SSCP screening of individual aptamers. Biotechniques, 2001. 31:828, 30, 32, 34.

230. Tsai, D.E., D.J. Kenan, and J.D. Keene, In vitro selection of an RNA epitope immunologically cross-reactive with a peptide. Proc Natl Acad Sci U S A, 1992. 89:8864-8.

231. Hamm, J. and M. Fornerod, Anti-idiotype RNAs that mimic the leucine-rich nuclear export signal and specifically bind to CRM1/exportin 1. Chem Biol, 2000. 7:345-54.

232. Hamm, J., J. Huber, and R. Luhrmann, Anti-idiotype RNA selected with an anti-nuclear export signal antibody is actively transported in oocytes and inhibits Rev- and cap-dependent RNA export. Proc Natl Acad Sci U S A, 1997. 94:12839-44.

233. Doudna, J.A., T.R. Cech, and B.A. Sullenger, Selection of an RNA molecule that mimics a major autoantigenic epitope of human insulin receptor. Proc Natl Acad Sci U S A, 1995. 92:2355-9.

234. Lee, S.W. and B.A. Sullenger, Isolation of a nuclease-resistant decoy RNA that selectively blocks autoantibody binding to insulin receptors on human lymphocytes. J Exp Med, 1996. 184:315-24.

235. Lee, S.W. and B.A. Sullenger, Isolation of a nuclease-resistant decoy RNA that can protect human acetylcholine receptors from myasthenic antibodies. Nat Biotechnol, 1997. 15:41-5.

236. Hwang, B. and S.W. Lee, Improvement of RNA aptamer activity against myasthenic autoantibodies by extended sequence selection. Biochem Biophys Res Commun, 2002. 290:656-62.

237. Seo, H.-S. and S.-W. Lee, In vitro Selection of the 2-Fluoro-2-Deoxyribonucleotide Decoy RNA Inhibitor of Myasthenic Autoantibodies. J. Microbiol. Biotechnol., 2000. 10:707-13.

238. Missailidis, S., D. Thomaidou, K.E. Borbas, and M.R. Price, Selection of aptamers with high affinity and high specificity against C595, an anti-MUC1 IgG3 monoclonal antibody, for antibody targeting. J Immunol Methods, 2005. 296:45-62.

239. Wiegand, T.W., P.B. Williams, S.C. Dreskin, M.H. Jouvin, J.P. Kinet, and D. Tasset, Highaffinity oligonucleotide ligands to human IgE inhibit binding to Fc epsilon receptor I. J Immunol, 1996. 157:221-30. 
240. Kim, Y.M., K.H. Choi, Y.J. Jang, J. Yu, and S. Jeong, Specific modulation of the anti-DNA autoantibody-nucleic acids interaction by the high affinity RNA aptamer. Biochem Biophys Res Commun, 2003. 300:516-23.

241. Kraus, E., W. James, and A.N. Barclay, Cutting edge: novel RNA ligands able to bind CD4 antigen and inhibit CD4+ T lymphocyte function. J Immunol, 1998. 160:5209-12.

242. Hui, Y., L. Shan, Z. Lin-Fu, and Z. Jian-Hua, Selection of DNA aptamers against DC-SIGN protein. Mol Cell Biochem, 2007. 306:71-7.

243. Ogawa, A., N. Tomita, N. Kikuchi, S. Sando, and Y. Aoyama, Aptamer selection for the inhibition of cell adhesion with fibronectin as target. Bioorg Med Chem Lett, 2004. 14:40014.

244. Cui, Y., P. Rajasethupathy, and G.P. Hess, Selection of stable RNA molecules that can regulate the channel-opening equilibrium of the membrane-bound gamma-aminobutyric acid receptor. Biochemistry, 2004. 43:16442-9.

245. Chen, C.H., G.A. Chernis, V.Q. Hoang, and R. Landgraf, Inhibition of heregulin signaling by an aptamer that preferentially binds to the oligomeric form of human epidermal growth factor receptor-3. Proc Natl Acad Sci U S A, 2003. 100:9226-31.

246. Mi, J., X. Zhang, P.H. Giangrande, J.O. McNamara, 2nd, S.M. Nimjee, S. Sarraf-Yazdi, B.A. Sullenger, and B.M. Clary, Targeted inhibition of alphavbeta3 integrin with an RNA aptamer impairs endothelial cell growth and survival. Biochem Biophys Res Commun, 2005. 338:956-63.

247. Ruckman, J., L. Gold, A. Stephens, N. Janjic, R. Rabin, and M. Lochrie, Nucleic acid ligands to hepatocyte growth factor/scatter factor (hgf/sf) or its receptor c-met and to integrins, World Intellectual Property Organization, Patent

248. Moreno, M., E. Rincon, D. Pineiro, G. Fernandez, A. Domingo, A. Jimenez-Ruiz, M. Salinas, and V.M. Gonzalez, Selection of aptamers against KMP-11 using colloidal gold during the SELEX process. Biochem Biophys Res Commun, 2003. 308:214-8.

249. Ferreira, C.S., K. Papamichael, G. Guilbault, T. Schwarzacher, J. Gariepy, and S. Missailidis, DNA aptamers against the MUC1 tumour marker: design of aptamer-antibody sandwich ELISA for the early diagnosis of epithelial tumours. Anal Bioanal Chem, 2007.

250. Ulrich, H., J.E. Ippolito, O.R. Pagan, V.A. Eterovic, R.M. Hann, H. Shi, J.T. Lis, M.E. Eldefrawi, and G.P. Hess, In vitro selection of RNA molecules that displace cocaine from the membrane-bound nicotinic acetylcholine receptor. Proc Natl Acad Sci U S A, 1998. 95:14051-6.

251. Cui, Y., H. Ulrich, and G.P. Hess, Selection of 2'-fluoro-modified RNA aptamers for alleviation of cocaine and MK-801 inhibition of the nicotinic acetylcholine receptor. J Membr Biol, 2004. 202:137-49.

252. Daniels, D.A., A.K. Sohal, S. Rees, and R. Grisshammer, Generation of RNA aptamers to the G-protein-coupled receptor for neurotensin, NTS-1. Anal Biochem, 2002. 305:214-26.

253. Lupold, S.E., B.J. Hicke, Y. Lin, and D.S. Coffey, Identification and characterization of nuclease-stabilized RNA molecules that bind human prostate cancer cells via the prostatespecific membrane antigen. Cancer Res, 2002. 62:4029-33.

254. Mori, T., A. Oguro, T. Ohtsu, and Y. Nakamura, RNA aptamers selected against the receptor activator of NF-kappaB acquire general affinity to proteins of the tumor necrosis factor receptor family. Nucleic Acids Res, 2004. 32:6120-8.

255. Jenison, R.D., S.D. Jennings, D.W. Walker, R.F. Bargatze, and D. Parma, Oligonucleotide inhibitors of P-selectin-dependent neutrophil-platelet adhesion. Antisense Nucleic Acid Drug Dev, 1998. 8:265-79.

256. Hicke, B.J., S.R. Watson, A. Koenig, C.K. Lynott, R.F. Bargatze, Y.F. Chang, S. Ringquist, L. Moon-McDermott, S. Jennings, T. Fitzwater, H.L. Han, N. Varki, I. Albinana, M.C. Willis, 
A. Varki, and D. Parma, DNA aptamers block L-selectin function in vivo. Inhibition of human lymphocyte trafficking in SCID mice. J Clin Invest, 1996. 98:2688-92.

257. O'Connell, D., A. Koenig, S. Jennings, B. Hicke, H.L. Han, T. Fitzwater, Y.F. Chang, N. Varki, D. Parma, and A. Varki, Calcium-dependent oligonucleotide antagonists specific for L-selectin. Proc Natl Acad Sci U S A, 1996. 93:5883-7.

258. Hicke, B.J., C. Marion, Y.F. Chang, T. Gould, C.K. Lynott, D. Parma, P.G. Schmidt, and S. Warren, Tenascin-C aptamers are generated using tumor cells and purified protein. J Biol Chem, 2001. 276:48644-54.

259. Daniels, D.A., H. Chen, B.J. Hicke, K.M. Swiderek, and L. Gold, A tenascin-C aptamer identified by tumor cell SELEX: systematic evolution of ligands by exponential enrichment. Proc Natl Acad Sci U S A, 2003. 100:15416-21.

260. Gal, S.W., S. Amontov, P.T. Urvil, D. Vishnuvardhan, F. Nishikawa, P.K. Kumar, and S. Nishikawa, Selection of a RNA aptamer that binds to human activated protein C and inhibits its protease function. Eur J Biochem, 1998. 252:553-62.

261. Rusconi, C.P., A. Yeh, H.K. Lyerly, J.H. Lawson, and B.A. Sullenger, Blocking the initiation of coagulation by RNA aptamers to factor VIIa. Thromb Haemost, 2000. 84:841-8.

262. Bless, N.M., D. Smith, J. Charlton, B.J. Czermak, H. Schmal, H.P. Friedl, and P.A. Ward, Protective effects of an aptamer inhibitor of neutrophil elastase in lung inflammatory injury. Curr Biol, 1997. 7:877-80.

263. Bridonneau, P., Y.F. Chang, D. O'Connell, S.C. Gill, D.W. Snyder, L. Johnson, T. Goodson, Jr., D.K. Herron, and D.H. Parma, High-affinity aptamers selectively inhibit human nonpancreatic secretory phospholipase A2 (hnps-PLA2). J Med Chem, 1998. 41:778-86.

264. Kumar, P.K., K. Machida, P.T. Urvil, N. Kakiuchi, D. Vishnuvardhan, K. Shimotohno, K. Taira, and S. Nishikawa, Isolation of RNA aptamers specific to the NS3 protein of hepatitis C virus from a pool of completely random RNA. Virology, 1997. 237:270-82.

265. Urvil, P.T., N. Kakiuchi, D.M. Zhou, K. Shimotohno, P.K. Kumar, and S. Nishikawa, Selection of RNA aptamers that bind specifically to the NS3 protease of hepatitis C virus. Eur J Biochem, 1997. 248:130-8.

266. Fukuda, K., D. Vishnuvardhan, S. Sekiya, J. Hwang, N. Kakiuchi, K. Taira, K. Shimotohno, P.K. Kumar, and S. Nishikawa, Isolation and characterization of RNA aptamers specific for the hepatitis C virus nonstructural protein 3 protease. Eur J Biochem, 2000. 267:3685-94.

267. Nishikawa, S., F. Nishikawa, and K. Fukuda, In vitro selection of RNA aptamers against HCV-NS3 helicase and their structural similarity with 3'(+)UTR of HCV. Nucleic Acids Res Suppl, 2003:241-2.

268. Nishikawa, F., K. Funaji, K. Fukuda, and S. Nishikawa, In vitro selection of RNA aptamers against the HCV NS3 helicase domain. Oligonucleotides, 2004. 14:114-29.

269. Conrad, R., L.M. Keranen, A.D. Ellington, and A.C. Newton, Isozyme-specific inhibition of protein kinase C by RNA aptamers. J Biol Chem, 1994. 269:32051-4.

270. Mallikaratchy, P., R.V. Stahelin, Z. Cao, W. Cho, and W. Tan, Selection of DNA ligands for protein kinase C-delta. Chem Commun (Camb), 2006:3229-31.

271. Skrypina, N.A., L.P. Savochkina, and R. Beabealashvilli, In vitro selection of single-stranded DNA aptamers that bind human pro-urokinase. Nucleosides Nucleotides Nucleic Acids, 2004. 23:891-3.

272. Bianchini, M., M. Radrizzani, M.G. Brocardo, G.B. Reyes, C. Gonzalez Solveyra, and T.A. Santa-Coloma, Specific oligobodies against ERK-2 that recognize both the native and the denatured state of the protein. J Immunol Methods, 2001. 252:191-7.

273. Bell, S.D., J.M. Denu, J.E. Dixon, and A.D. Ellington, RNA molecules that bind to and inhibit the active site of a tyrosine phosphatase. J Biol Chem, 1998. 273:14309-14. 
274. Kimoto, M., M. Shirouzu, S. Mizutani, H. Koide, Y. Kaziro, I. Hirao, and S. Yokoyama, Anti-(Raf-1) RNA aptamers that inhibit Ras-induced Raf-1 activation. Eur J Biochem, 2002. 269:697-704.

275. Takeno, H., S. Yamamoto, T. Tanaka, Y. Sakano, and Y. Kikuchi, Selection of an RNA molecule that specifically inhibits the protease activity of subtilisin. J Biochem (Tokyo), 1999. 125:1115-9.

276. Bock, L.C., L.C. Griffin, J.A. Latham, E.H. Vermaas, and J.J. Toole, Selection of singlestranded DNA molecules that bind and inhibit human thrombin. Nature, 1992. 355:564-6.

277. Tasset, D.M., M.F. Kubik, and W. Steiner, Oligonucleotide inhibitors of human thrombin that bind distinct epitopes. J Mol Biol, 1997. 272:688-98.

278. Kubik, M.F., A.W. Stephens, D. Schneider, R.A. Marlar, and D. Tasset, High-affinity RNA ligands to human alpha-thrombin. Nucleic Acids Res, 1994. 22:2619-26.

279. Liu, X., D. Zhang, G. Cao, G. Yang, H. Ding, G. Liu, M. Fan, B. Shen, and N. Shao, RNA aptamers specific for bovine thrombin. J Mol Recognit, 2003. 16:23-7.

280. White, R.R., S. Shan, C.P. Rusconi, G. Shetty, M.W. Dewhirst, C.D. Kontos, and B.A. Sullenger, Inhibition of rat corneal angiogenesis by a nuclease-resistant RNA aptamer specific for angiopoietin-2. Proc Natl Acad Sci U S A, 2003. 100:5028-33.

281. Jellinek, D., C.K. Lynott, D.B. Rifkin, and N. Janjic, High-affinity RNA ligands to basic fibroblast growth factor inhibit receptor binding. Proc Natl Acad Sci U S A, 1993. 90:1122731.

282. Jellinek, D., L.S. Green, C. Bell, C.K. Lynott, N. Gill, C. Vargeese, G. Kirschenheuter, D.P. McGee, P. Abesinghe, W.A. Pieken, and et al., Potent 2'-amino-2'-deoxypyrimidine RNA inhibitors of basic fibroblast growth factor. Biochemistry, 1995. 34:11363-72.

283. Saito, T. and M. Tomida, Generation of inhibitory DNA aptamers against human hepatocyte growth factor. DNA Cell Biol, 2005. 24:624-33.

284. Lin, Y., D. Nieuwlandt, A. Magallanez, B. Feistner, and S.D. Jayasena, High-affinity and specific recognition of human thyroid stimulating hormone (hTSH) by in vitro-selected 2'amino-modified RNA. Nucleic Acids Res, 1996. 24:3407-14.

285. Padmanabhan, K., K.P. Padmanabhan, J.D. Ferrara, J.E. Sadler, and A. Tulinsky, The structure of alpha-thrombin inhibited by a 15-mer single-stranded DNA aptamer. J Biol Chem, 1993. 268:17651-4.

286. Schultze, P., R.F. Macaya, and J. Feigon, Three-dimensional solution structure of the thrombin-binding DNA aptamer d(GGTTGGTGTGGTTGG). J Mol Biol, 1994. 235:1532-47.

287. Martino, L., A. Virno, A. Randazzo, A. Virgilio, V. Esposito, C. Giancola, M. Bucci, G. Cirino, and L. Mayol, A new modified thrombin binding aptamer containing a 5'-5' inversion of polarity site. Nucleic Acids Res, 2006. 34:6653-62.

288. Marathias, V.M., K.Y. Wang, S. Kumar, T.Q. Pham, S. Swaminathan, and P.H. Bolton, Determination of the number and location of the manganese binding sites of DNA quadruplexes in solution by EPR and NMR in the presence and absence of thrombin. J Mol Biol, 1996. 260:378-94.

289. Mao, X., L.A. Marky, and W.H. Gmeiner, NMR structure of the thrombin-binding DNA aptamer stabilized by Sr2+. J Biomol Struct Dyn, 2004. 22:25-33.

290. Binkley, J., P. Allen, D.M. Brown, L. Green, C. Tuerk, and L. Gold, RNA ligands to human nerve growth factor. Nucleic Acids Res, 1995. 23:3198-205.

291. Rhodes, A., A. Deakin, J. Spaull, B. Coomber, A. Aitken, P. Life, and S. Rees, The generation and characterization of antagonist RNA aptamers to human oncostatin M. J Biol Chem, 2000. 275:28555-61.

292. Green, L.S., D. Jellinek, R. Jenison, A. Ostman, C.H. Heldin, and N. Janjic, Inhibitory DNA ligands to platelet-derived growth factor B-chain. Biochemistry, 1996. 35:14413-24. 
293. Yan, X., X. Gao, and Z. Zhang, Isolation and characterization of 2'-amino-modified RNA aptamers for human TNFalpha. Genomics Proteomics Bioinformatics, 2004. 2:32-42.

294. Jellinek, D., L.S. Green, C. Bell, and N. Janjic, Inhibition of receptor binding by high-affinity RNA ligands to vascular endothelial growth factor. Biochemistry, 1994. 33:10450-6.

295. Ruckman, J., L.S. Green, J. Beeson, S. Waugh, W.L. Gillette, D.D. Henninger, L. ClaessonWelsh, and N. Janjic, 2'-Fluoropyrimidine RNA-based aptamers to the 165-amino acid form of vascular endothelial growth factor (VEGF165). Inhibition of receptor binding and VEGFinduced vascular permeability through interactions requiring the exon 7 -encoded domain. $\mathbf{J}$ Biol Chem, 1998. 273:20556-67.

296. Chen, H. and L. Gold, Selection of high-affinity RNA ligands to reverse transcriptase: inhibition of cDNA synthesis and RNase H activity. Biochemistry, 1994. 33:8746-56.

297. Shi, H., B.E. Hoffman, and J.T. Lis, A specific RNA hairpin loop structure binds the RNA recognition motifs of the Drosophila SR protein B52. Mol Cell Biol, 1997. 17:2649-57.

298. Hirao, I., Y. Harada, T. Nojima, Y. Osawa, H. Masaki, and S. Yokoyama, In vitro selection of RNA aptamers that bind to colicin E3 and structurally resemble the decoding site of $16 \mathrm{~S}$ ribosomal RNA. Biochemistry, 2004. 43:3214-21.

299. Gening, L.V., S.A. Klincheva, A. Reshetnjak, A.P. Grollman, and H. Miller, RNA aptamers selected against DNA polymerase beta inhibit the polymerase activities of DNA polymerases beta and kappa. Nucleic Acids Res, 2006. 34:2579-86.

300. Ishizaki, J., J.R. Nevins, and B.A. Sullenger, Inhibition of cell proliferation by an RNA ligand that selectively blocks E2F function. Nat Med, 1996. 2:1386-9.

301. Nazarenko, I.A. and O.C. Uhlenbeck, Defining a smaller RNA substrate for elongation factor Tu. Biochemistry, 1995. 34:2545-52.

302. Oguro, A., T. Ohtsu, Y.V. Svitkin, N. Sonenberg, and Y. Nakamura, RNA aptamers to initiation factor 4A helicase hinder cap-dependent translation by blocking ATP hydrolysis. Rna, 2003. 9:394-407.

303. Mochizuki, K., A. Oguro, T. Ohtsu, N. Sonenberg, and Y. Nakamura, High affinity RNA for mammalian initiation factor 4E interferes with mRNA-cap binding and inhibits translation. Rna, 2005. 11:77-89.

304. Miyakawa, S., A. Oguro, T. Ohtsu, H. Imataka, N. Sonenberg, and Y. Nakamura, RNA aptamers to mammalian initiation factor $4 \mathrm{G}$ inhibit cap-dependent translation by blocking the formation of initiation factor complexes. Rna, 2006. 12:1825-34.

305. Chen, H., D.G. McBroom, Y.Q. Zhu, L. Gold, and T.W. North, Inhibitory RNA ligand to reverse transcriptase from feline immunodeficiency virus. Biochemistry, 1996. 35:6923-30.

306. Wen, J.D., C.W. Gray, and D.M. Gray, SELEX selection of high-affinity oligonucleotides for bacteriophage Ff gene 5 protein. Biochemistry, 2001. 40:9300-10.

307. Jones, L.A., L.E. Clancy, W.D. Rawlinson, and P.A. White, High-affinity aptamers to subtype 3a hepatitis $\mathrm{C}$ virus polymerase display genotypic specificity. Antimicrob Agents Chemother, 2006. 50:3019-27.

308. Vo, N.V., J.W. Oh, and M.M. Lai, Identification of RNA ligands that bind hepatitis C virus polymerase selectively and inhibit its RNA synthesis from the natural viral RNA templates. Virology, 2003. 307:301-16.

309. Elenbaas, B., M. Dobbelstein, J. Roth, T. Shenk, and A.J. Levine, The MDM2 oncoprotein binds specifically to RNA through its RING finger domain. Mol Med, 1996. 2:439-51.

310. Sakamoto, T., A. Oguro, G. Kawai, T. Ohtsu, and Y. Nakamura, NMR structures of double loops of an RNA aptamer against mammalian initiation factor 4A. Nucleic Acids Res, 2005. 33:745-54. 
311. Andreola, M.L., F. Pileur, C. Calmels, M. Ventura, L. Tarrago-Litvak, J.J. Toulme, and S. Litvak, DNA aptamers selected against the HIV-1 RNase H display in vitro antiviral activity. Biochemistry, 2001. 40:10087-94.

312. Levine, T.D., F. Gao, P.H. King, L.G. Andrews, and J.D. Keene, Hel-N1: an autoimmune RNA-binding protein with specificity for 3' uridylate-rich untranslated regions of growth factor mRNAs. Mol Cell Biol, 1993. 13:3494-504.

313. Allen, P., S. Worland, and L. Gold, Isolation of high-affinity RNA ligands to HIV-1 integrase from a random pool. Virology, 1995. 209:327-36.

314. de Soultrait, V.R., P.Y. Lozach, R. Altmeyer, L. Tarrago-Litvak, S. Litvak, and M.L. Andreola, DNA aptamers derived from HIV-1 RNase $\mathrm{H}$ inhibitors are strong anti-integrase agents. J Mol Biol, 2002. 324:195-203.

315. Yamamoto, R., M. Katahira, S. Nishikawa, T. Baba, K. Taira, and P.K. Kumar, A novel RNA motif that binds efficiently and specifically to the Ttat protein of HIV and inhibits the transactivation by Tat of transcription in vitro and in vivo. Genes Cells, 2000. 5:371-88.

316. Kim, S.J., M.Y. Kim, J.H. Lee, J.C. You, and S. Jeong, Selection and stabilization of the RNA aptamers against the human immunodeficiency virus type-1 nucleocapsid protein. Biochem Biophys Res Commun, 2002. 291:925-31.

317. Allen, P., B. Collins, D. Brown, Z. Hostomsky, and L. Gold, A specific RNA structural motif mediates high affinity binding by the HIV-1 nucleocapsid protein (NCp7). Virology, 1996. 225:306-15.

318. Lochrie, M.A., S. Waugh, D.G. Pratt, Jr., J. Clever, T.G. Parslow, and B. Polisky, In vitro selection of RNAs that bind to the human immunodeficiency virus type-1 gag polyprotein. Nucleic Acids Res, 1997. 25:2902-10.

319. Giver, L., D. Bartel, M. Zapp, A. Pawul, M. Green, and A.D. Ellington, Selective optimization of the Rev-binding element of HIV-1. Nucleic Acids Res, 1993. 21:5509-16.

320. Jensen, K.B., L. Green, S. MacDougal-Waugh, and C. Tuerk, Characterization of an in vitroselected RNA ligand to the HIV-1 Rev protein. J Mol Biol, 1994. 235:237-47.

321. $\mathrm{Xu}, \mathrm{W}$. and A.D. Ellington, Anti-peptide aptamers recognize amino acid sequence and bind a protein epitope. Proc Natl Acad Sci U S A, 1996. 93:7475-80.

322. Tuerk, C., S. MacDougal, and L. Gold, RNA pseudoknots that inhibit human immunodeficiency virus type 1 reverse transcriptase. Proc Natl Acad Sci U S A, 1992. 89:6988-92.

323. Schneider, D.J., J. Feigon, Z. Hostomsky, and L. Gold, High-affinity ssDNA inhibitors of the reverse transcriptase of type 1 human immunodeficiency virus. Biochemistry, 1995. 34:9599610.

324. Bryant, K.F., J.C. Cox, H. Wang, J.M. Hogle, A.D. Ellington, and D.M. Coen, Binding of herpes simplex virus-1 US11 to specific RNA sequences. Nucleic Acids Res, 2005. 33:6090100.

325. Kumarevel, T.S., S.C. Gopinath, S. Nishikawa, H. Mizuno, and P.K. Kumar, Identification of important chemical groups of the hut mRNA for HutP interactions that regulate the hut operon in Bacillus subtilis. Nucleic Acids Res, 2004. 32:3904-12.

326. Goodman, S.D., N.J. Velten, Q. Gao, S. Robinson, and A.M. Segall, In vitro selection of integration host factor binding sites. J Bacteriol, 1999. 181:3246-55.

327. Yoo, S. and W.S. Dynan, Characterization of the RNA binding properties of Ku protein. Biochemistry, 1998. 37:1336-43.

328. Dobbelstein, M. and T. Shenk, In vitro selection of RNA ligands for the ribosomal L22 protein associated with Epstein-Barr virus-expressed RNA by using randomized and cDNAderived RNA libraries. J Virol, 1995. 69:8027-34. 
329. Surugiu-Warnmark, I., A. Warnmark, G. Toresson, J.A. Gustafsson, and L. Bulow, Selection of DNA aptamers against rat liver X receptors. Biochem Biophys Res Commun, 2005. 332:512-7.

330. He, Y.Y., P.G. Stockley, and L. Gold, In vitro evolution of the DNA binding sites of Escherichia coli methionine repressor, MetJ. J Mol Biol, 1996. 255:55-66.

331. McGregor, A., J.B. Murray, C.J. Adams, P.G. Stockley, and B.A. Connolly, Secondary structure mapping of an RNA ligand that has high affinity for the MetJ repressor protein and interference modification analysis of the protein-RNA complex. J Biol Chem, 1999. 274:2255-62.

332. Ghisolfi-Nieto, L., G. Joseph, F. Puvion-Dutilleul, F. Amalric, and P. Bouvet, Nucleolin is a sequence-specific RNA-binding protein: characterization of targets on pre-ribosomal RNA. J Mol Biol, 1996. 260:34-53.

333. Bae, S.J., J.H. Oum, S. Sharma, J. Park, and S.W. Lee, In vitro selection of specific RNA inhibitors of NFATc. Biochem Biophys Res Commun, 2002. 298:486-92.

334. Lebruska, L.L. and L.J. Maher, 3rd, Selection and characterization of an RNA decoy for transcription factor NF-kappa B. Biochemistry, 1999. 38:3168-74.

335. Wurster, S.E. and L.J. Maher, 3rd, Selection and characterization of anti-NF-kappaB p65 RNA aptamers. Rna, 2008. 14:1037-47.

336. Schneider, D., L. Gold, and T. Platt, Selective enrichment of RNA species for tight binding to Escherichia coli rho factor. Faseb J, 1993. 7:201-7.

337. Phan, A.T., V. Kuryavyi, J.B. Ma, A. Faure, M.L. Andreola, and D.J. Patel, An interlocked dimeric parallel-stranded DNA quadruplex: a potent inhibitor of HIV-1 integrase. Proc Natl Acad Sci U S A, 2005. 102:634-9.

338. Matsugami, A., S. Kobayashi, K. Ouhashi, S. Uesugi, R. Yamamoto, K. Taira, S. Nishikawa, P.K. Kumar, and M. Katahira, Structural basis of the highly efficient trapping of the HIV Tat protein by an RNA aptamer. Structure, 2003. 11:533-45.

339. Ye, X., A. Gorin, A.D. Ellington, and D.J. Patel, Deep penetration of an alpha-helix into a widened RNA major groove in the HIV-1 rev peptide-RNA aptamer complex. Nat Struct Biol, 1996. 3:1026-33.

340. Ye, X., A. Gorin, R. Frederick, W. Hu, A. Majumdar, W. Xu, G. McLendon, A. Ellington, and D.J. Patel, RNA architecture dictates the conformations of a bound peptide. Chem Biol, 1999. 6:657-69.

341. Jaeger, J., T. Restle, and T.A. Steitz, The structure of HIV-1 reverse transcriptase complexed with an RNA pseudoknot inhibitor. Embo J, 1998. 17:4535-42.

342. Bouvet, P., F.H. Allain, L.D. Finger, T. Dieckmann, and J. Feigon, Recognition of preformed and flexible elements of an RNA stem-loop by nucleolin. J Mol Biol, 2001. 309:76375.

343. Huang, D.B., D. Vu, L.A. Cassiday, J.M. Zimmerman, L.J. Maher, 3rd, and G. Ghosh, Crystal structure of NF-kappaB (p50)2 complexed to a high-affinity RNA aptamer. Proc Natl Acad Sci U S A, 2003. 100:9268-73.

344. Reiter, N.J., L.J. Maher, 3rd, and S.E. Butcher, DNA mimicry by a high-affinity anti-NF\{kappa\}B RNA aptamer. Nucleic Acids Res, 2007.

345. Jiang, F., A. Gorin, W. Hu, A. Majumdar, S. Baskerville, W. Xu, A. Ellington, and D.J. Patel, Anchoring an extended HTLV-1 Rex peptide within an RNA major groove containing junctional base triples. Structure, 1999. 7:1461-72.

346. Dang, C. and S.D. Jayasena, Oligonucleotide inhibitors of Taq DNA polymerase facilitate detection of low copy number targets by PCR. J Mol Biol, 1996. 264:268-78.

347. Sando, S., A. Ogawa, T. Nishi, M. Hayami, and Y. Aoyama, In vitro selection of RNA aptamer against Escherichia coli release factor 1. Bioorg Med Chem Lett, 2007. 17:1216-20. 
348. Pileur, F., M.L. Andreola, E. Dausse, J. Michel, S. Moreau, H. Yamada, S.A. Gaidamakov, R.J. Crouch, J.J. Toulme, and C. Cazenave, Selective inhibitory DNA aptamers of the human RNase H1. Nucleic Acids Res, 2003. 31:5776-88.

349. Kulbachinskiy, A., A. Feklistov, I. Krasheninnikov, A. Goldfarb, and V. Nikiforov, Aptamers to Escherichia coli core RNA polymerase that sense its interaction with rifampicin, sigmasubunit and GreB. Eur J Biochem, 2004. 271:4921-31.

350. Thomas, M., S. Chedin, C. Carles, M. Riva, M. Famulok, and A. Sentenac, Selective targeting and inhibition of yeast RNA polymerase II by RNA aptamers. J Biol Chem, 1997. 272:27980-6.

351. Ringquist, S., T. Jones, E.E. Snyder, T. Gibson, I. Boni, and L. Gold, High-affinity RNA ligands to Escherichia coli ribosomes and ribosomal protein S1: comparison of natural and unnatural binding sites. Biochemistry, 1995. 34:3640-8.

352. Klug, S.J., A. Huttenhofer, M. Kromayer, and M. Famulok, In vitro and in vivo characterization of novel mRNA motifs that bind special elongation factor SelB. Proc Natl Acad Sci U S A, 1997. 94:6676-81.

353. Fan, X., H. Shi, K. Adelman, and J.T. Lis, Probing TBP interactions in transcription initiation and reinitiation with RNA aptamers that act in distinct modes. Proc Natl Acad Sci U S A, 2004. 101:6934-9.

354. Shi, H., X. Fan, A. Sevilimedu, and J.T. Lis, RNA aptamers directed to discrete functional sites on a single protein structural domain. Proc Natl Acad Sci U S A, 2007. 104:3742-6.

355. Lee, S.Y. and S. Jeong, In vitro selection and characterization of TCF-1 binding RNA aptamers. Mol Cells, 2004. 17:174-9.

356. Lee, S.K., M.W. Park, E.G. Yang, J. Yu, and S. Jeong, An RNA aptamer that binds to the beta-catenin interaction domain of TCF-1 protein. Biochem Biophys Res Commun, 2005. 327:294-9.

357. Neely, L.S., B.M. Lee, J. Xu, P.E. Wright, and J.M. Gottesfeld, Identification of a minimal domain of $5 \mathrm{~S}$ ribosomal RNA sufficient for high affinity interactions with the RNA-specific zinc fingers of transcription factor IIIA. J Mol Biol, 1999. 291:549-60.

358. Brunel, C., B. Ehresmann, C. Ehresmann, and M. McKeown, Selection of genomic target RNAs by iterative screening. Bioorg Med Chem, 2001. 9:2533-41.

359. Hale, S.P. and P. Schimmel, Protein synthesis editing by a DNA aptamer. Proc Natl Acad Sci U S A, 1996. 93:2755-8.

360. Bullock, T.L., L.D. Sherlin, and J.J. Perona, Tertiary core rearrangements in a tight binding transfer RNA aptamer. Nat Struct Biol, 2000. 7:497-504.

361. Murphy, M.B., S.T. Fuller, P.M. Richardson, and S.A. Doyle, An improved method for the in vitro evolution of aptamers and applications in protein detection and purification. Nucleic Acids Res, 2003. 31:e110.

362. Lin, Y. and S.D. Jayasena, Inhibition of multiple thermostable DNA polymerases by a heterodimeric aptamer. J Mol Biol, 1997. 271:100-11.

363. Tsai, D.E., D.S. Harper, and J.D. Keene, U1-snRNP-A protein selects a ten nucleotide consensus sequence from a degenerate RNA pool presented in various structural contexts. Nucleic Acids Res, 1991. 19:4931-6.

364. Bardeesy, N. and J. Pelletier, Overlapping RNA and DNA binding domains of the wt1 tumor suppressor gene product. Nucleic Acids Res, 1998. 26:1784-92.

365. Zhai, G., M. Iskandar, K. Barilla, and P.J. Romaniuk, Characterization of RNA aptamer binding by the Wilms' tumor suppressor protein WT1. Biochemistry, 2001. 40:2032-40.

366. Kettenberger, H., A. Eisenfuhr, F. Brueckner, M. Theis, M. Famulok, and P. Cramer, Structure of an RNA polymerase II-RNA inhibitor complex elucidates transcription regulation by noncoding RNAs. Nat Struct Mol Biol, 2006. 13:44-8. 
367. Weiss, S., D. Proske, M. Neumann, M.H. Groschup, H.A. Kretzschmar, M. Famulok, and E.L. Winnacker, RNA aptamers specifically interact with the prion protein PrP. J Virol, 1997. 71:8790-7.

368. Takemura, K., P. Wang, I. Vorberg, W. Surewicz, S.A. Priola, A. Kanthasamy, R. Pottathil, S.G. Chen, and S. Sreevatsan, DNA aptamers that bind to $\operatorname{PrP}(C)$ and not $\operatorname{PrP}(\mathrm{Sc})$ show sequence and structure specificity. Exp Biol Med (Maywood), 2006. 231:204-14.

369. Rhie, A., L. Kirby, N. Sayer, R. Wellesley, P. Disterer, I. Sylvester, A. Gill, J. Hope, W. James, and A. Tahiri-Alaoui, Characterization of 2'-fluoro-RNA aptamers that bind preferentially to disease-associated conformations of prion protein and inhibit conversion. $\mathbf{J}$ Biol Chem, 2003. 278:39697-705.

370. Tang, J., T. Yu, L. Guo, J. Xie, N. Shao, and Z. He, In vitro selection of DNA aptamer against abrin toxin and aptamer-based abrin direct detection. Biosens Bioelectron, 2007. 22:2456-63.

371. Bruno, J.G. and J.L. Kiel, Use of magnetic beads in selection and detection of biotoxin aptamers by electrochemiluminescence and enzymatic methods. Biotechniques, 2002. 32:178-80, 82-3.

372. Hirao, I., K. Madin, Y. Endo, S. Yokoyama, and A.D. Ellington, RNA aptamers that bind to and inhibit the ribosome-inactivating protein, pepocin. J Biol Chem, 2000. 275:4943-8.

373. Hesselberth, J.R., D. Miller, J. Robertus, and A.D. Ellington, In vitro selection of RNA molecules that inhibit the activity of ricin A-chain. J Biol Chem, 2000. 275:4937-42.

374. Tang, J., J. Xie, N. Shao, and Y. Yan, The DNA aptamers that specifically recognize ricin toxin are selected by two in vitro selection methods. Electrophoresis, 2006. 27:1303-11.

375. Purschke, W.G., F. Radtke, F. Kleinjung, and S. Klussmann, A DNA Spiegelmer to staphylococcal enterotoxin B. Nucleic Acids Res, 2003. 31:3027-32.

376. Houser-Scott, F., P. Ansel-McKinney, J.M. Cai, and L. Gehrke, In vitro genetic selection analysis of alfalfa mosaic virus coat protein binding to 3'-terminal AUGC repeats in the viral RNAs. J Virol, 1997. 71:2310-9.

377. Boyce, M., F. Scott, L.M. Guogas, and L. Gehrke, Base-pairing potential identified by in vitro selection predicts the kinked RNA backbone observed in the crystal structure of the alfalfa mosaic virus RNA-coat protein complex. J Mol Recognit, 2006. 19:68-78.

378. Hirao, I., M. Spingola, D. Peabody, and A.D. Ellington, The limits of specificity: an experimental analysis with RNA aptamers to MS2 coat protein variants. Mol Divers, 1998. 4:75-89.

379. Lim, F. and D.S. Peabody, RNA recognition site of PP7 coat protein. Nucleic Acids Res, 2002. 30:4138-44.

380. Schneider, D., C. Tuerk, and L. Gold, Selection of high affinity RNA ligands to the bacteriophage R17 coat protein. J Mol Biol, 1992. 228:862-9.

381. Lee, H.K., Y.S. Choi, Y.A. Park, and S. Jeong, Modulation of oncogenic transcription and alternative splicing by beta-catenin and an RNA aptamer in colon cancer cells. Cancer Res, 2006. 66:10560-6.

382. Biesecker, G., L. Dihel, K. Enney, and R.A. Bendele, Derivation of RNA aptamer inhibitors of human complement C5. Immunopharmacology, 1999. 42:219-30.

383. Mayer, G., M. Blind, W. Nagel, T. Bohm, T. Knorr, C.L. Jackson, W. Kolanus, and M. Famulok, Controlling small guanine-nucleotide-exchange factor function through cytoplasmic RNA intramers. Proc Natl Acad Sci U S A, 2001. 98:4961-5.

384. Guogas, L.M., D.J. Filman, J.M. Hogle, and L. Gehrke, Cofolding organizes alfalfa mosaic virus RNA and coat protein for replication. Science, 2004. 306:2108-11.

385. Horn, W.T., M.A. Convery, N.J. Stonehouse, C.J. Adams, L. Liljas, S.E. Phillips, and P.G. Stockley, The crystal structure of a high affinity RNA stem-loop complexed with the 
bacteriophage MS2 capsid: further challenges in the modeling of ligand-RNA interactions. Rna, 2004. 10:1776-82.

386. Convery, M.A., S. Rowsell, N.J. Stonehouse, A.D. Ellington, I. Hirao, J.B. Murray, D.S. Peabody, S.E. Phillips, and P.G. Stockley, Crystal structure of an RNA aptamer-protein complex at 2.8 A resolution. Nat Struct Biol, 1998. 5:133-9.

387. Rowsell, S., N.J. Stonehouse, M.A. Convery, C.J. Adams, A.D. Ellington, I. Hirao, D.S. Peabody, P.G. Stockley, and S.E. Phillips, Crystal structures of a series of RNA aptamers complexed to the same protein target. Nat Struct Biol, 1998. 5:970-5.

388. Khati, M., M. Schuman, J. Ibrahim, Q. Sattentau, S. Gordon, and W. James, Neutralization of infectivity of diverse R5 clinical isolates of human immunodeficiency virus type 1 by gp120binding 2'F-RNA aptamers. J Virol, 2003. 77:12692-8.

389. Dey, A.K., M. Khati, M. Tang, R. Wyatt, S.M. Lea, and W. James, An aptamer that neutralizes R5 strains of human immunodeficiency virus type 1 blocks gp120-CCR5 interaction. J Virol, 2005. 79:13806-10.

390. Dey, A.K., C. Griffiths, S.M. Lea, and W. James, Structural characterization of an anti-gp120 RNA aptamer that neutralizes R5 strains of HIV-1. Rna, 2005. 11:873-84.

391. Misono, T.S. and P.K. Kumar, Selection of RNA aptamers against human influenza virus hemagglutinin using surface plasmon resonance. Anal Biochem, 2005. 342:312-7.

392. Jeon, S.H., B. Kayhan, T. Ben-Yedidia, and R. Arnon, A DNA aptamer prevents influenza infection by blocking the receptor binding region of the viral hemagglutinin. J Biol Chem, 2004. 279:48410-9.

393. Gopinath, S.C., Y. Sakamaki, K. Kawasaki, and P.K. Kumar, An efficient RNA aptamer against human influenza B virus hemagglutinin. J Biochem (Tokyo), 2006. 139:837-46.

394. Zhao, X., H. Shi, A. Sevilimedu, N. Liachko, H.C. Nelson, and J.T. Lis, An RNA aptamer that interferes with the DNA binding of the HSF transcription activator. Nucleic Acids Res, 2006. 34:3755-61.

395. Rhodes, A., N. Smithers, T. Chapman, S. Parsons, and S. Rees, The generation and characterisation of antagonist RNA aptamers to MCP-1. FEBS Lett, 2001. 506:85-90.

396. Tahiri-Alaoui, A., L. Frigotto, N. Manville, J. Ibrahim, P. Romby, and W. James, High affinity nucleic acid aptamers for streptavidin incorporated into bi-specific capture ligands. Nucleic Acids Res, 2002. 30:e45.

397. Srisawat, C. and D.R. Engelke, Streptavidin aptamers: affinity tags for the study of RNAs and ribonucleoproteins. Rna, 2001. 7:632-41.

398. Stoltenburg, R., C. Reinemann, and B. Strehlitz, FluMag-SELEX as an advantageous method for DNA aptamer selection. Anal Bioanal Chem, 2005. 383:83-91.

399. Tian, Y., N. Adya, S. Wagner, C.Z. Giam, M.R. Green, and A.D. Ellington, Dissecting protein:protein interactions between transcription factors with an RNA aptamer. Rna, 1995. $1: 317-26$.

400. Bridonneau, P., Y.F. Chang, A.V. Buvoli, D. O'Connell, and D. Parma, Site-directed selection of oligonucleotide antagonists by competitive elution. Antisense Nucleic Acid Drug Dev, 1999. 9:1-11.

401. Ko, J., Y. Lee, I. Park, and B. Cho, Identification of a structural motif of 23S rRNA interacting with 5S rRNA. FEBS Lett, 2001. 508:300-4.

402. Tok, J.B., J. Cho, and R.R. Rando, RNA aptamers that specifically bind to a 16 S ribosomal RNA decoding region construct. Nucleic Acids Res, 2000. 28:2902-10.

403. Pei, D.H., H.D. Ulrich, and P.G. Schultz, A combinatorial approach toward DNA recognition. Science, 1991. 253:1408-11.

404. Soukup, G.A., A.D. Ellington, and L.J. Maher, 3rd, Selection of RNAs that bind to duplex DNA at neutral pH. J Mol Biol, 1996. 259:216-28. 
405. Le Tinevez, R., R.K. Mishra, and J.J. Toulme, Selective inhibition of cell-free translation by oligonucleotides targeted to a mRNA hairpin structure. Nucleic Acids Res, 1998. 26:2273-8.

406. Mishra, R.K., R. Le Tinevez, and J.J. Toulme, Targeting nucleic acid secondary structures by antisense oligonucleotides designed through in vitro selection. Proc Natl Acad Sci U S A, 1996. 93:10679-84.

407. Kikuchi, K., T. Umehara, K. Fukuda, J. Hwang, A. Kuno, T. Hasegawa, and S. Nishikawa, RNA aptamers targeted to domain II of hepatitis $C$ virus IRES that bind to its apical loop region. J Biochem (Tokyo), 2003. 133:263-70.

408. Da Rocha Gomes, S., E. Dausse, and J.J. Toulme, Determinants of apical loop-internal loop RNA-RNA interactions involving the HCV IRES. Biochem Biophys Res Commun, 2004. 322:820-6.

409. Kikuchi, K., T. Umehara, K. Fukuda, A. Kuno, T. Hasegawa, and S. Nishikawa, A hepatitis $\mathrm{C}$ virus (HCV) internal ribosome entry site (IRES) domain III-IV-targeted aptamer inhibits translation by binding to an apical loop of domain IIId. Nucleic Acids Res, 2005. 33:683-92.

410. Aldaz-Carroll, L., B. Tallet, E. Dausse, L. Yurchenko, and J.J. Toulme, Apical loop-internal loop interactions: a new RNA-RNA recognition motif identified through in vitro selection against RNA hairpins of the hepatitis C virus mRNA. Biochemistry, 2002. 41:5883-93.

411. Lodmell, J.S., C. Ehresmann, B. Ehresmann, and R. Marquet, Convergence of natural and artificial evolution on an RNA loop-loop interaction: the HIV-1 dimerization initiation site. Rna, 2000. 6:1267-76.

412. Boiziau, C., E. Dausse, L. Yurchenko, and J.J. Toulme, DNA aptamers selected against the HIV-1 trans-activation-responsive RNA element form RNA-DNA kissing complexes. J Biol Chem, 1999. 274:12730-7.

413. Sekkai, D., E. Dausse, C. Di Primo, F. Darfeuille, C. Boiziau, and J.J. Toulme, In vitro selection of DNA aptamers against the HIV-1 TAR RNA hairpin. Antisense Nucleic Acid Drug Dev, 2002. 12:265-74.

414. Darfeuille, F., D. Sekkai, E. Dausse, G. Kolb, L. Yurchenko, C. Boiziau, and J.J. Toulme, Driving in vitro selection of anti-HIV-1 TAR aptamers by magnesium concentration and temperature. Comb Chem High Throughput Screen, 2002. 5:313-25.

415. Duconge, F. and J.J. Toulme, In vitro selection identifies key determinants for loop-loop interactions: RNA aptamers selective for the TAR RNA element of HIV-1. Rna, 1999. 5:1605-14.

416. Scarabino, D., A. Crisari, S. Lorenzini, K. Williams, and G.P. Tocchini-Valentini, tRNA prefers to kiss. Embo J, 1999. 18:4571-8.

417. Fauzi, H., K.D. Jack, and J.V. Hines, In vitro selection to identify determinants in tRNA for Bacillus subtilis tyrS T box antiterminator mRNA binding. Nucleic Acids Res, 2005. 33:2595-602.

418. Manimala, J.C., S.L. Wiskur, A.D. Ellington, and E.V. Anslyn, Tuning the specificity of a synthetic receptor using a selected nucleic acid receptor. J Am Chem Soc, 2004. 126:16515-9.

419. Bruno, J.G. and J.L. Kiel, In vitro selection of DNA aptamers to anthrax spores with electrochemiluminescence detection. Biosens Bioelectron, 1999. 14:457-64.

420. Wang, C., M. Zhang, G. Yang, D. Zhang, H. Ding, H. Wang, M. Fan, B. Shen, and N. Shao, Single-stranded DNA aptamers that bind differentiated but not parental cells: subtractive systematic evolution of ligands by exponential enrichment. J Biotechnol, 2003. 102:15-22.

421. Cerchia, L., F. Duconge, C. Pestourie, J. Boulay, Y. Aissouni, K. Gombert, B. Tavitian, V. de Franciscis, and D. Libri, Neutralizing aptamers from whole-cell SELEX inhibit the RET receptor tyrosine kinase. PLoS Biol, 2005. 3:e123. 
422. Gopinath, S.C., T.S. Misono, K. Kawasaki, T. Mizuno, M. Imai, T. Odagiri, and P.K. Kumar, An RNA aptamer that distinguishes between closely related human influenza viruses and inhibits haemagglutinin-mediated membrane fusion. J Gen Virol, 2006. 87:479-87.

423. Lee, Y.J. and S.-W. Lee, In Vitro Selection of Cancer-Specific RNA Aptamers. J. Microbiol. Biotechnol., 2006. 16:1149-53.

424. Ennifar, E., P. Walter, B. Ehresmann, C. Ehresmann, and P. Dumas, Crystal structures of coaxially stacked kissing complexes of the HIV-1 RNA dimerization initiation site. Nat Struct Biol, 2001. 8:1064-8.

425. Mujeeb, A., J.L. Clever, T.M. Billeci, T.L. James, and T.G. Parslow, Structure of the dimer initiation complex of HIV-1 genomic RNA. Nat Struct Biol, 1998. 5:432-6.

426. Lebars, I., T. Richard, C. Di Primo, and J.J. Toulme, NMR structure of a kissing complex formed between the TAR RNA element of HIV-1 and a LNA-modified aptamer. Nucleic Acids Res, 2007. 35:6103-14.

427. Shangguan, D., Y. Li, Z. Tang, Z.C. Cao, H.W. Chen, P. Mallikaratchy, K. Sefah, C.J. Yang, and W. Tan, Aptamers evolved from live cells as effective molecular probes for cancer study. Proc Natl Acad Sci U S A, 2006. 103:11838-43.

428. Chen, F., J. Zhou, F. Luo, A.B. Mohammed, and X.L. Zhang, Aptamer from whole-bacterium SELEX as new therapeutic reagent against virulent Mycobacterium tuberculosis. Biochem Biophys Res Commun, 2007. 357:743-8.

429. Zhang, F. and D. Anderson, In vitro selection of bacteriophage phi29 prohead RNA aptamers for prohead binding. J Biol Chem, 1998. 273:2947-53.

430. Brown, D. and L. Gold, Template recognition by an RNA-dependent RNA polymerase: identification and characterization of two RNA binding sites on Q beta replicase. Biochemistry, 1995. 34:14765-74.

431. Mallikaratchy, P., Z. Tang, L. Meng, D. Shangguan, S. Kwame, and W. Tan, Aptamer directly evolved from live cells recognizes membrane bound immunoglobin heavy mu chain in Burkitt's lymphoma cells. Mol Cell Proteomics, 2007.

432. Tang, Z., D. Shangguan, K. Wang, H. Shi, K. Sefah, P. Mallikratchy, H.W. Chen, Y. Li, and W. Tan, Selection of aptamers for molecular recognition and characterization of cancer cells. Anal Chem, 2007. 79:4900-7.

433. Pan, W., R.C. Craven, Q. Qiu, C.B. Wilson, J.W. Wills, S. Golovine, and J.F. Wang, Isolation of virus-neutralizing RNAs from a large pool of random sequences. Proc Natl Acad Sci U S A, 1995. 92:11509-13.

434. Morris, K.N., K.B. Jensen, C.M. Julin, M. Weil, and L. Gold, High affinity ligands from in vitro selection: complex targets. Proc Natl Acad Sci U S A, 1998. 95:2902-7.

435. Yao, W., K. Adelman, and J.A. Bruenn, In vitro selection of packaging sites in a doublestranded RNA virus. J Virol, 1997. 71:2157-62.

436. Ulrich, H., M.H. Magdesian, M.J. Alves, and W. Colli, In vitro selection of RNA aptamers that bind to cell adhesion receptors of Trypanosoma cruzi and inhibit cell invasion. J Biol Chem, 2002. 277:20756-62.

437. Homann, M. and H.U. Goringer, Combinatorial selection of high affinity RNA ligands to live African trypanosomes. Nucleic Acids Res, 1999. 27:2006-14.

438. Lorger, M., M. Engstler, M. Homann, and H.U. Goringer, Targeting the variable surface of African trypanosomes with variant surface glycoprotein-specific, serum-stable RNA aptamers. Eukaryot Cell, 2003. 2:84-94.

439. Blank, M., T. Weinschenk, M. Priemer, and H. Schluesener, Systematic evolution of a DNA aptamer binding to rat brain tumor microvessels. selective targeting of endothelial regulatory protein pigpen. J Biol Chem, 2001. 276:16464-8. 


\title{
CHAPTER 3. STERIC PROTECTION OF MOLECULAR REACTIVITY BY
}

\author{
AN APTAMER \\ Tianjiao Wang ${ }^{1}$, Julie Hoy ${ }^{1}$, Monica Lamm ${ }^{2}$ and Marit Nilsen-Hamilton ${ }^{1}$ \\ From: ${ }^{1}$ Department of Biochemistry, Biophysics and Molecular Biology, and ${ }^{2}$ Department of \\ Chemical and Biological Engineering, Ames, IA 50011 \\ Address correspondence to: Marit Nilsen-Hamilton, 3206 Mol Biol Blding, Dept of BBMB, Iowa \\ State University, Ames, IA 50011; FAX: 515-294-0453; Email: marit@iastate.edu
}

\begin{abstract}
Living organisms have evolved protection mechanisms against many reactive small molecules that are undesirable and even lethal. These mechanisms range from catalytic inactivation to ejection through membrane efflux pumps. We have examined another means of controlling the reactivity of small molecules, which is steric protection by a nucleic acid aptamer. We used the malachite green aptamer (MGA) as a model system and have shown that the MGA can control the rate of bleaching of its target molecule, malachite green (MG). Bleaching occurs by a pH-dependent second order reaction in which an $\mathrm{OH}^{-}$attacks the central (C1) carbon of $\mathrm{MG}$ to form a carbinol base. While bound to the MGA, MG bleaching is prevented. The MGA effect is reversed by an antisense oligonucleotide complementary to the MGA binding pocket. Computational cavity analysis of the NMR structure of the MGA-MG complex predicted that the $\mathrm{OH}^{-}$is sterically excluded from the $\mathrm{C} 1$ of MG in the complex. These predictions were confirmed experimentally using variants of the MGA with changes in the MG binding pocket. This work shows that a nucleic acid aptamer can utilize steric hindrance to control access to specific chemical groups on small molecules. This finding has potential for in vivo applications such as in controlling metabolic reactions and protein modifications by small reactants.
\end{abstract}

\section{INTRODUCTION}


Aptamers are short single-stranded nucleic acids that can bind to their targets with high specificity and high affinity and can be selected in vitro to recognize a large range of types and sizes of molecular targets (1). The related ribozymes have the additional ability to perform chemical catalysis. However, the distinction between aptamers and ribozyme is blurred when it comes to catalysis. Although aptamers are selected for their binding abilities, some perform catalytic functions. Several catalytic aptamers have been isolated by selecting for their ability to bind a stable transition state analog. One example is an RNA aptamer that promotes isomerization of its target molecule, a bridged biphenyl, with a $\mathrm{K}_{\mathrm{cat}} / \mathrm{K}_{\mathrm{m}}$ of $8.7 \times 10^{-4} \mathrm{M}^{-1} \mathrm{~S}^{-1}(2)$. DNA and RNA aptamers selected against $\mathrm{N}$-methylmesoporphyrin (NMMP), a stable transition state analog of porphyrin metalation, can insert $\mathrm{Cu}$ (II) into mesoporphyrin IX with $\mathrm{K}_{\mathrm{cat}} / \mathrm{K}_{\mathrm{m}}$ 's of $0.13 \mathrm{M}^{-1} \mathrm{~S}^{-1}$ and $2100 \mathrm{M}^{-1} \mathrm{~S}^{-1}$ respectively $(3,4)$. The DNA aptamer selected for NMMP also binds hemin and the hemin-DNA aptamer complex has peroxidase activity with $\mathrm{K}_{\mathrm{cat}} / \mathrm{K}_{\mathrm{m}}$ of $278 \mathrm{M}^{-1} \mathrm{~S}^{-1}(5)$.

Even when not selected against a transition state analog, the structures of some aptamer targets can be distorted when bound by the aptamer, which may lower the energy barrier to chemical conversion. For example, a DNA aptamer selected to bind sulforhodamine B catalyzes the oxidation of dihydrotetramethylrosamine to tetramethylrosamine, albeit at a very slow rate (6). Another example is the malachite green aptamer (MGA), which enhances the rate of hydrolysis of a malachite green acetate ester by 1000 -fold (7).

The MGA binds its target, malachite green $(\mathrm{MG})$, with a reasonably high affinity $\left(\mathrm{K}_{\mathrm{D}} \sim 1 \mu \mathrm{M}\right)$ and concurrently distorts the MG structure to flatten it. Binding by the MGA results in a large increase in fluorescence emission by MG (8).

The central carbon of $\mathrm{MG}$ is susceptible to attack by a base such as $\mathrm{OH}^{-}$, which results in the formation of a colorless form (MG-OH). We show here that, whereas $\mathrm{MG}$ readily reacts with $\mathrm{OH}^{-}$in solution the presence of stoichiometric proportions of the MGA inhibits the formation of MG-OH. 
Inhibition of this reaction by the MGA requires structural integrity of the MG-binding pocket of the MGA because inhibition is reversed by an oligonucleotide complementary to the binding pocket.

Computational analysis of the NMR structure of the MGA-MG complex showed no possible route for an $\mathrm{OH}^{-}$of a minimum possible size to reach the reactive central carbon of MG. The computational analysis was confirmed by mutational analysis of the MGA and structural probing of the MGA variants.

These studies show that an aptamer can fold to fit around its target so perfectly that even a small molecule the size of a hydroxyl group cannot penetrate the complex. This observation suggests that nucleic acids could play a role in vivo to protect sensitive metabolites or proteins from modification by small reactive molecules.

\section{EXPERIMENTAL PROCEDURES}

Chemicals and Oligonucleotides- Malachite green oxalate (MG) was purchased from Sigma. MG carbinol base (MG-OH) was prepared as follows: A $90 \mathrm{ml}$ solution of $17 \mathrm{mg} / \mathrm{ml} \mathrm{MG}$ in $0.5 \mathrm{M}$ HEPES, $\mathrm{pH} 7.4$ was left at $23^{\circ} \mathrm{C}$ for $24 \mathrm{~h}$. The precipitated product was centrifuged at $1500 \mathrm{~g}$ for $10 \mathrm{~min}$. The pellet was collected then dried at $74{ }^{\circ} \mathrm{C}$ for $6 \mathrm{~h}$. All $\mathrm{pH}$ 's reported here were measured at room temperature $\left(\sim 23^{\circ} \mathrm{C}\right)$

Oligonucleotides were synthesized by Integrated DNA Technologies, Inc. (Coralville, IA) or by the DNA Synthesis and Sequencing Facility at Iowa State University as listed here. MGA (9): GGAUCCCGACUGGCGAGAGCCAGGUAACGAAUGGAUCC, MGA (U25C): GGAUCCCGACUGGCGAGAGCCAGGCAACGAAUGGAUCC, MGA(A31C): GGAUCCCGACUGGCGAGAGCCAGGUAACGAC̣UGGAUCC, MGA(G8C, G24C, G29C): GGAUCCCCAACUGGCGAGAGCCAGC्UAACĆAAUGGAUCC, ${ }^{1}$ AS: GATCCATTCGTTACCTGGC and Shuffled AS: TCTCTAGAGTCTCTGCACG.

\footnotetext{
${ }^{1}$ AS: antisense oligonucleotide targets the region of MGA from G19 to C37
} 
In addition to chemical synthesis, in vitro transcription by T7 RNA polymerase was also used to generate a 100 nt random RNA whose sequence was_GGGAGACAAGAAUAAACGCUCAA(N53)UUCGACAGGAGGCUCACAACAGGC

Mass Spectrometry- Electrospray ionization of $1 \mathrm{mM} \mathrm{MG}$ in $\mathrm{ddH}_{2} \mathrm{O}$ was performed with a Finnigan TSQ700 triple quadrupole mass spectrometer (Finnigan MAT, San Jose, CA) fitted with a Finnigan ESI interface. The sample was introduced into the electrospray interface through an untreated fusedsilica capillary with a $50 \mu \mathrm{m}$ i.d. and $190 \mu \mathrm{m}$ o.d. A mixture of $75 \mu \mathrm{g} / \mathrm{ml}$ horse skeletal muscle myoglobin and $12 \mu \mathrm{g} / \mathrm{ml}$ Met-Arg-Phe-Ala (MRFA) tetrapeptide in a 50:50 methanol:water solution was used for tuning and routine calibration of the instrument. The tuning mixture in a polypropylene vial was infused into the mass spectrometer at a rate of $3 \mu \mathrm{l} / \mathrm{min}$ on a Harvard Apparatus (model 22, South Natick, MA) syringe pump.

Electron impact ionization of MG-OH was performed on a TSQ700 triple quadrupole mass spectrometer (Finnigan MAT, San Jose, CA) fitted with a Finnigan EI/CI ion source. The sample was introduced into the mass spectrometer using the solids probe, which was heated gradually from 100 to 400 degrees. The instrument was used as a single quadrupole and scanned from 35 to 650 Daltons.

NMR Spectrometry- Solid state ${ }^{13} \mathrm{C}-\mathrm{NMR}$ spectra of $\mathrm{MG}$ and $\mathrm{MG}-\mathrm{OH}$ were recorded at room temperature at $150 \mathrm{MHz}$ by a Bruker AV-600 with CPTOSS as the pulse program.

${ }^{1} \mathrm{H}-\mathrm{NMR}$ spectra were acquired of samples with $50 \mu \mathrm{M}$ MGA $\pm 100 \mu \mathrm{M}$ MG; $50 \mu \mathrm{M}$ $\operatorname{MGA}(\mathrm{A} 31 \mathrm{C}) \pm 100 \mu \mathrm{M}$ MG; $50 \mu \mathrm{M}$ MGA(U25C) $\pm 200 \mu \mathrm{M}$ MG; $50 \mu \mathrm{M}$ MGA(G8C-G24C-G29C) $\pm 500 \mu \mathrm{M}$ MG and $100 \mu \mathrm{M}$ MG in $10 \mathrm{mM} \mathrm{KH}_{2} \mathrm{PO}_{4}, 5 \mathrm{mM} \mathrm{MgCl} 2,10 \mathrm{mM} \mathrm{KCl}, \mathrm{pH} 5.8,5 \% \mathrm{D}_{2} \mathrm{O} /$ 95\% $\mathrm{H}_{2} \mathrm{O}$. Spectra were collected using a Bruker Avance 700 spectrometers equipped with a $5 \mathrm{~mm}$ HCN-Z gradient cryoprobe. All 1D ${ }^{1} \mathrm{H}-\mathrm{NMR}$ spectra were acquired using a WATERGATE pulse sequence with water flipback (Bruker sequence p3919fpgp) to minimize solvent saturation transfer. Spectra were averages of 512 transients. Spectral width was $18182 \mathrm{~Hz}(25 \mathrm{ppm})$. 
Affinities of MGA Variants for MG- The binding of the MGA variants to MG causes the $\lambda_{\max }$ of MG absorption to shift from $618 \mathrm{~nm}$ to between $629-632 \mathrm{~nm}$ depending on the variant (9). The binding also enhances MG fluorescence (8). Based on these properties, the affinities of the MGA variants were determined by UV-Visible spectroscopy either using a Biowave S2100 Diode Array Spectrophotometer (WPA) or a ND-1000 Spectrophotometer (NanoDrop) or by a fluorescence spectrophotometer (Cary Eclipse, Variant, Palo Alto, CA). The E coli tRNA (Roche, Indianapolis, IN) and AS were used as controls for the effects of RNAs that interact with MG nonspecifically.

The UV-visible spectra were obtained of duplicated samples of MG and each MGA and its variants with at least 6 different concentrations of each MGA and its variants. The concentrations of the MGA and variants were adjusted to ensure saturation of MG in each case. The $\lambda_{\max }$ for each scan was recorded and plotted against the concentration of the appropriate MGA variant or control RNAs, AS or tRNA. The plots were fitted to $\lambda=\lambda_{0}+\Delta \lambda[\mathrm{M}] /\left(\mathrm{K}_{\mathrm{D}}+[\mathrm{M}]\right)$ using a nonlinear regression function in the Costat program (CoHort Software, CA), where $\lambda$ is the maximal absorption wavelength of MG in the presence of different concentrations of the MGA variant, AS or tRNA, $\lambda_{0}$ is the maximal absorption wavelength of free MG, $\Delta \lambda$ is the difference of maximal absorption wavelengths between free MG and MG saturated with each alternate RNA, $K_{D}$ is the dissociation constant and $[M]$ is the concentration of the RNA.

To obtain $\mathrm{K}_{\mathrm{D}} \mathrm{s}$ of the MGA and its variants, and MG, the fluorescence emission spectra for MG were determined in $100 \mathrm{mM}$ Tris, $100 \mathrm{mM} \mathrm{KCl}, 5 \mathrm{mM} \mathrm{MgCl}_{2}, \mathrm{pH} 9.0$ and in the presence of each of 11 different concentrations of the MGA variants. The samples were excited at $630 \mathrm{~nm}$ and the emission spectra were recorded from 640-700 nm with $650 \mathrm{~nm}$ as the maximal excitation wavelength. The data were fit to $\mathrm{F}_{650} / \mathrm{F}_{\max }=[\mathrm{M}] /\left(\mathrm{K}_{\mathrm{D}}+[\mathrm{M}]\right)$ using the Costat program (CoHort Software, $\left.\mathrm{CA}\right)$ where $F_{650}$ is the fluorescence intensity at $650 \mathrm{~nm}$ of samples, $F_{\max }$ is the $F_{650}$ when MG is completely saturated, $[\mathrm{M}]$ is the concentrations of the MGA variants and $K_{D}$ is the dissociation constant for MG and the MGA variants. 
Kinetics of MG-OH formation- The UV-visible spectra of MG with or without each MGA variant, AS or shuffled AS were determined by using either a Biowave S2100 Diode Array Spectrophotometer (WPA) or a ND-1000 Spectrophotometer (NanoDrop). Three independent experiments were done of each of the described conditions. The rates of production of MG-OH were monitored for up to $46 \mathrm{~h}$ by recording $\mathrm{A}\left(\lambda_{\max }\right)$. The $\lambda_{\max }$ ranged from $618 \mathrm{~nm}$ to $632 \mathrm{~nm}$ depending on the concentration and the MGA variants. Then $\mathrm{A}\left(\lambda_{\max }\right)$ was plotted against time and fitted by a nonlinear regression function in Costat to the equation, $\mathrm{A}=\mathrm{A}_{0} /\left(1+\mathrm{ktC} \mathrm{C}_{0}\right)$, in which $\mathrm{A}_{0}$ is the $\mathrm{A}\left(\lambda_{\max }\right)$ at $\mathrm{t}=0, \mathrm{~A}$ is the $\mathrm{A}\left(\lambda_{\max }\right)$ at each time point, $\mathrm{k}$ is the reaction rate, $\mathrm{t}$ is the time, and $\mathrm{C}_{0}$ is the initial concentration of MG. The half life of MG-OH formation was calculated as $\mathrm{t}_{1 / 2}=1 / \mathrm{kC}_{0}$.

Cavity Analysis of Free MG and the MG-MGA Complex- Analysis of the NMR structure of the MGA (1q8n.pdb) by LSQMAN (10) indicates that the central chain is E and the average RMSD between chains $=0.709 \AA$ (using $\mathrm{C} 4 * \mathrm{P} \mathrm{C} 1 * \mathrm{C} 2 * \mathrm{C} 3 * \mathrm{O} 2 * \mathrm{O} 3 * \mathrm{O} 4 *$ atoms to align chains). Chain $\mathrm{N}$ has the highest deviation from chain E with the RMSD between chains $=0.870 \AA$, thus both chains $\mathrm{E}$ and $\mathrm{N}$ were analyzed for cavities. Because no important difference was found between these two chains, only the results for chain $\mathrm{E}$ are reported. Cavity analysis of the MGA binding pockets and free MG were performed using SURFNET (11). A radius of $1.32 \AA$ was chosen as the smallest possible radius for a hydroxide ion (12) while the maximum radius was set to $3 \AA$. The figure was created with SPDBV (13) and POV-Ray (Persistence of Vision Raytracer Pty. Ltd.).

Structure probing of the MGA variants- RNase I footprinting was used to study the structural alterations of the binding pocket of the MGA due to mutations (A31C; U25C and G8C-G24C- G29C). RNase I can cleave RNA in single-stranded but not in double-stranded regions. The MGA variants, including MGA, MGA(A31C), MGA(U25C) and MGA(G8C-G24C-G29C), were first labeled with $\gamma$ ${ }^{32}$ P-ATP (MP Biomedicals, Solon, OH) by T4 polynucleotide kinase (Promega, Madison, WI) at their 5'-ends. Twenty $\mu$ l labeling reaction mixtures containing $5 \mu \mathrm{M}$ RNA, $1.5 \mu \mathrm{Ci} / \mu \mathrm{l} \gamma-{ }^{32} \mathrm{P}$-ATP, $1 \mathrm{U} / \mu \mathrm{l}$ T4 polynucleotide kinase, $70 \mathrm{mM}$ Tris- $\mathrm{HCl}$ (pH 7.6), $10 \mathrm{mM} \mathrm{MgCl}$ and $5 \mathrm{mM}$ DTT were incubated 
for $1.5 \mathrm{~h}$ at $37^{\circ} \mathrm{C}$. The labeled MGA variants were purified by electrophoresis through $10 \%$ polyacrylamide gels in the presence of $7 \mathrm{M}$ urea, then incubated with RNase I in the presence or absence of MG to probe the RNA structure. Reaction mixtures of $10 \mu$ containing $0.125 \mu \mathrm{M}$ ${ }^{32}$ P-labeled RNA, $0.5 \times 10^{-4} \mathrm{U} / \mu 1$ RNase I, $100 \mathrm{mM} \mathrm{KCl}, 5 \mathrm{mM} \mathrm{MgCl} 2,10 \mathrm{mM}$ HEPES, pH 7.4, were incubated at $23^{\circ} \mathrm{C}$ for $10 \mathrm{~min}$ in the absence or presence of MG. Partial alkaline hydrolysis of the labeled RNAs was done in $50 \mu \mathrm{M} \mathrm{Na} \mathrm{CO}_{3}, \mathrm{pH} 9.0$ at $95^{\circ} \mathrm{C}$ for 5 min. RNase T1 digestion of the labeled RNAs was carried out in $10 \mu \mathrm{l}$ reaction containing $0.5-1 \mathrm{U} / \mu \mathrm{l}$ Rase T1, $5 \mathrm{M}$ urea, $350 \mathrm{mM}$ sodium citrate, $0.7 \mathrm{mM}$ EDTA, $\mathrm{pH} 5.0$, at $50^{\circ} \mathrm{C}$ for $4 \mathrm{~min}$. The RNA hydrolysis and cleavage reactions were stopped by bringing the reaction mixtures to $47.5 \%$ formamide, $0.05 \%$ bromophenol blue, and $0.05 \%$ xylene cyanol FF. The samples were resolved by electrophoresis through $10 \%$ polyacrylamide gels in the presence of $7 \mathrm{M}$ urea. The radioactivity was recorded from the dried gels using a phosphor screen and imaged with a Typhoon 8600 Variable Model Imager (GE Healthcare).

\section{RESULTS}

Conversion of $M G$ to $M G-O H$. MG loses color by a mechanism that is $\mathrm{pH}$-dependent and follows second order kinetics (Fig. 1). The chemical form of the decolorized MG was characterized by mass spectrometry and NMR spectroscopy. The mass spectrum of MG had an expected peak at $329 \mathrm{~m} / \mathrm{z}$ and the MG-OH spectrum contained an additional $346 \mathrm{~m} / \mathrm{z}$ peak as expected of hydroxylated MG (Fig 2A). In the NMR spectra of $\mathrm{MG}$ and $\mathrm{MG}-\mathrm{OH}$, the $\mathrm{C} 1$ peak was shifted from the $175.91 \mathrm{ppm}$ in MG to $82.59 \mathrm{ppm}$ in MG-OH. The $\mathrm{C} 11$ of $\mathrm{MG}$ at $165.115 \mathrm{ppm}$ was shifted to $149.08 \mathrm{ppm}$ in MG$\mathrm{OH}$ and the double peaks of $\mathrm{N}\left(\mathrm{CH}_{3}\right)_{2}$ and $+\mathrm{N}\left(\mathrm{CH}_{3}\right)_{2}(40.91 \mathrm{ppm}$ and $39.95 \mathrm{ppm})$ in MG became a single peak of $\mathrm{N}\left(\mathrm{CH}_{3}\right)_{2}(40.39 \mathrm{ppm})$ in $\mathrm{MG}-\mathrm{OH}$ (Fig 2B). These results are consistent with the conclusion that MG-OH is hydroxylated at $\mathrm{C} 1$ and that the decolorized $\mathrm{MG}$ is in the form of a carbinol base (Fig 2C). 
The Malachite Green Aptamer Binding Pocket Protects MG from Conversion to MG-OH. With the route of $\mathrm{OH}^{-}$attack on $\mathrm{MG}$ being limited by the equatorial phenyl rings, anion attack can only proceed by polar routes (Fig. 4A). Because the MG binding pocket is formed by bases that lie above and below MG, it seemed possible that the MGA might further protect MG from conversion to MG$\mathrm{OH}$ by sterically hindering the entrance of $\mathrm{OH}^{-}$by the polar routes. We tested this hypothesis by incubating MG with various concentrations of the MGA and found that the MGA inhibited MG-OH formation with a dependence on MGA concentration (Fig. 3A, B). At $6 \mu \mathrm{M}$ MGA, a concentration that is about 6 times the $\mathrm{K}_{\mathrm{D}}$ for the MGA-MG interaction, the rate of MG conversion was almost zero. By contrast, $10 \mu \mathrm{M} 100 \mathrm{nt}$ random RNA had no effect on the rate of MG to MG-OH conversion (Fig. $3 \mathrm{~A}, \mathrm{~B})$.

If the MGA protects the MG in its binding pocket from hydroxyl attack by steric hindrance, then a distortion of the binding pocket should result in loss of its effect on MG bleaching. This result was observed in experiments in which the reaction mixture included an oligonucleotide (AS) with a sequence complementary to bases 19 to 37 in the MGA sequence, which constitute one half of the MG-binding pocket. Addition of AS (but not its shuffled sequence version) to a mixture of MGA and MG reversed the protection by MGA of MG bleaching with 30\% reversal at 2AS/MGA and almost complete reversal at 10AS/MGA. ( Fig. 3 C,D).

Cavity Analysis of the MGA-MG Complex Shows No Gap for OH Entry. Our results suggested that the ability of the MGA to inhibit hydroxylation of MG is due to the configuration of the MGMGA complex that sterically hinders the $\mathrm{OH}^{-}$access to the $\mathrm{C} 1$ of the $\mathrm{MG}$ located in the binding pocket. Due to the very small size of $\mathrm{OH}^{-}$, this condition requires a very snug fit of $\mathrm{MG}$ in the binding pocket of the MGA. Cavity analysis was used to test the hypothesis. Whereas the $\mathrm{C} 1$ of free MG is accessible from the poles (Fig. 4B,C), cavity analysis of MG sitting in the MGA binding pocket showed that MG was protected from polar attack by G8-C28 and C7-G29-G24-A31, which 
does not allow a gap of the minimum size of an $\mathrm{OH}^{-}$group to reach $\mathrm{C} 1$ from the solvent surface of the MGA (Fig. 4D).

Mutations in the MGA Binding Pocket Compromise the Ability of the MGA to Protect MG Conversion To MG-OH. To test the hypothesis that steric hindrance in the MGA binding pocket prevents hydroxyl attack, variants of the MGA with mutations around the binding pocket were made including MGA(A31C), MGA(U25C) and MGA(G8C-G24C-G29C) based on cavity analysis and their locations in the binding pockets.

Based on the measured $\mathrm{K}_{\mathrm{D}}$ value of each MGA variant (Table I), a concentration of each MGA variant equivalent to 10-15 times of its $K_{D}$ was chosen at which MG would be saturated by the RNA. Under these conditions the rate of MG bleaching was negligible in the presence of the MGA, intermediate for the MGA(A31C) and MGA(U25C) and large for the MGA(G8C-G24C-G29C) and tRNA (Fig. 5, Table I).

Structural Probing Shows Structurally Altered MG Binding Pockets in the MGA Variants. To determine whether there is structural change in the binding pocket of the MGA variants, RNase I footprinting was performed of the MGA variants in the presence of various concentrations of MG. The data showed that MG binding to the MGA protected the binding pocket and destabilized the tetraloop (Fig. 6A). The MGA(A31C showed a similar cleavage pattern to the MGA except there was increased cleavage if U32 and C31 (Fig. 6B). In the MGA(U25C), exactly the same cleavage pattern was observed as for the MGA (Fig. 6C). By contrast, all regions of the MGA(G8C-G24CG29C) were protected by MG except for C29 (Fig. 6D).

${ }^{I} H$-NMR spectra showed proper folding of the MGA variants and binding of MG. To determine whether the MGA variants still folded properly and bound MG, ${ }^{1} \mathrm{H}-\mathrm{NMR}$ spectra were acquired for the MGA variants in the presence and absence of MG. The spectrum of MG alone was also acquired as a control, which showed sharp peaks in 6.8-6.9, 7.25-7.35, 7.45-7.50 and 7.60-7.65 ppm regions 
(Fig. 7). These peaks were similarly broadened in the presence of the MGA and all MGA variants, indicating a similar binding mode of MG to the MGA variants as for MGA.

The number of peaks from the MGA variants in the 10-15 ppm region, which reference the basepairings in the MGA structure, was 9-10 for all MGA variants in the absence of MG. In the presence of MG, there were 13-14 peaks for MGA, MGA(A31C) and MGA(U25C) and 7 for MGA(G8CG24C-G29C). These results are consistent with the conclusion that the MGA variants, fold properly into a structure similar to MGA and that can bind to MG.

In the presence of MG the spectra of the MGA and MGA variants differed, especially in the region of $6.5-9.0 \mathrm{ppm}$ region where the proton exchange rates are not affected by $\mathrm{pH}$, salt and other buffer conditions. We interpret these results to show that MGA and its variants fold into similar but nonidentical structures that can all bind MG.

\section{DISCUSSION}

MG is held tightly in a pocket of the MGA that is created by a bulge between two short stems resulting in a structurally modified MG (14). Our results show that this interaction is so snug that it prevents access of a molecule as small as a hydroxyl ion to the central carbon of MG.

We characterized the conversion of $\mathrm{MG}$ to $\mathrm{MG}-\mathrm{OH}$ by $\mathrm{UV}$-Visible spectrophotometry, mass spectrometry and NMR spectrometry. The results of these studies suggest that the most likely form of MG-OH contains a carbinol group at position $\mathrm{C} 1$. MG bleaching is also $\mathrm{pH}$-dependent and second order. Thus, it appears that the $\mathrm{C} 1$ of $\mathrm{MG}$ is attacked by an $\mathrm{OH}^{-}$to form $\mathrm{MG}-\mathrm{OH}$. This reaction product was also predicted in a study of MG bleaching in alkaline solution (15).

The MGA inhibited MG hydroxylation in a concentration-dependent manner with a stoichiometry that suggested steric hindrance rather than a catalytic mechanism. The specificity of the effect of the MGA on MG was demonstrated by the observation that high concentrations of a random RNA had no effect on MG hydroxylation (Fig. 3A,B). The ability of an oligonucleotide (AS), complementary to 
the binding pocket of the MGA, to reverse the effect of the MGA on MG bleaching (Fig 3C,D) showed that the MGA must bind MG to prevent hydroxylation. Similar reversibility has been shown in other systems in which the activities of aptamers are regulated $(16,17)$.

Several mechanisms were considered for how the MGA could protect $\mathrm{MG}$ from $\mathrm{OH}^{-}$attack. First, the MGA could alter the charge on $\mathrm{C} 1$ to make it less susceptible to $\mathrm{OH}^{-}$attack. The MGA has been shown to change the charge distribution across MG, but physical chemical calculations showed that $\mathrm{C} 1$ is more positively charged in the MG-MGA complex compared to in solution (14). A more positively charged MG would be more susceptible to $\mathrm{OH}^{-}$attack rather than less as we have observed. By this analysis, we excluded charge distribution as the mechanism by which the MGA prevents MG hydroxylation.

To test the hypothesis, that the MGA might protect MG from hydroxylation by steric hindrance. we first carried out a computational structural analysis of the MG-MGA complex (18) and free MG. The results of these studies showed that the rings $\mathrm{A}, \mathrm{B}$ and $\mathrm{C}$ of $\mathrm{MG}$ could protect $\mathrm{MG}$ from $\mathrm{OH}^{-}$ attack from the sides but allowed $\mathrm{OH}^{-}$attack from the poles at the top and bottom. Cavity analysis of the MG-MGA complex showed that the MGA binding pocket further protected $\mathrm{MG}$ from $\mathrm{OH}^{-}$attack from the poles. Thus, it appeared that the mechanism by which the MGA protects MG from hydroxylation was by steric hindrance (Fig. 4).

To confirm the prediction made by cavity analysis, we tested the effects of several MGA variants on MG hydroxylation. Mutations in the MGA binding pocket were chosen that might alter the pocket sufficiently to allow $\mathrm{OH}^{-}$access to $\mathrm{C} 1$ of $\mathrm{MG}$. The $\mathrm{A} 31 \mathrm{C}$ mutation changes part of the base quadruple (C7-G29-G24-A31) that sits above MG and the U25C mutation changes the U-turn connecting the top and bottom portions of the binding pocket. The G8C-G24C-G29C mutation changes the G8-C28 and C7-G29 base pairs between which MG intercalates and was expected to completely alter the binding pocket in the region where MG intercalates between the G8-C28 and C7G29 base pairs. 
One-dimensional NMR spectra of these RNAs and the footprinting results suggested that the pockets remained intact albeit alterations in structure. The ${ }^{1} \mathrm{H}-\mathrm{NMR}$ spectra of the MGA and all its variants were consistent with their proper folding. In the absence of MG these RNAs showed 9-10 peaks in the $10-15$ ppm region, which corresponds to $\geq 9-10$ base pairings in the MGA variants. This is consistent with the reported secondary structure of the MGA (18). On binding MG, the number of peaks in the 10-15 ppm region of the ${ }^{1} \mathrm{H}-\mathrm{NMR}$ spectra increased to $13-14$ in the MGA, MGA(A31C) and MGA(U25C) and remained at 7 in the MGA(G8C-G24C-G29C). These results are consistent with the RNase I footprinting results and lead to the interpretation that the MG-binding pocket is formed in the MGA, MGA(A31C), MGA(U25C) but not in the MGA(G8C-G24C-G29C) that binds only nonspecifically.

Structural changes in the binding pockets of the MGA variants are indicated by the following evidence: 1) enhanced RNase I cleavage of U32 and C31 in the MGA(A31C), 2) changed $\lambda$ max of the MG interaction with the MGA(U25C), 3) significantly altered RNase I footprint of the MGA(G8C-G24C-G29C) compared with the MGA.

The $\mathrm{K}_{\mathrm{D}} \mathrm{S}$ of the MGA variants for MG differed widely with the MGA(G8C-G24C-G29C) showing a significant loss of affinity for MG. Intermediate changes in the $\mathrm{K}_{\mathrm{D}} \mathrm{s}$ were observed for the A31C and U25C variants. Although A31 makes no direct contact with MG, the A31C mutation changed part of the base quadruple (C7-G29-G24-A31) that sits above MG in the MGA binding pocket. The U25C mutation changed the U-turn that connects the top and bottom parts of the binding pocket. These changes were not evident from the NMR structure.

With MG saturated by the concentration of each RNA at 10-15 times its $K_{D}$, the MGA(A31C), MGA(U25C) and MGA(G8C-G24C-G29C) protected MG from hydroxylation in decreasing order of effectiveness with the effect of the MGA(G8C-G24C-G29C) being the same as a nonspecific binder (tRNA). 
In conclusion, in this model system, we have shown that, when sequestered in the binding pocket of its cognate aptamer, MG can be protected from attack by a molecule as small as 1.32-3 angstroms. The use of steric hindrance to control protein function by aptamers has been reported for enzyme exosites (19-22), substrate binding sites (17,23-32), catalytic sites (33-35), and cellular localization sites (36). However, this is the first report to our knowledge of an aptamer targeted to a small molecule that can protect it from reacting with an even smaller hydroxyl group. This capability of an aptamer to wrap so snugly around its target as to prevent access of even small ions might find application in protecting small molecules from reactive species. The additional ability to regulate the aptamer structure with an oligonucleotide that is complementary to the aptamer binding pocket provides a means of rapidly releasing the sequestered small molecule. Aptamers can also function inside living cells. Thus, this novel finding has potential applications for regulating molecular reactivity of small molecules both in vitro and in vivo.

\section{REFERENCES}

1. Stoltenburg, R., Reinemann, C., and Strehlitz, B. (2007) Biomol Eng 24, 381-403

2. Prudent, J. R., Uno, T., and Schultz, P. G. (1994) Science 264, 1924-1927

3. Conn, M. M., Prudent, J. R., and Schultz, P. G. (1996) J Am Chem Soc 118, 7012-7013

4. $\quad$ Li, Y., and Sen, D. (1996) Nat Struct Biol 3, 743-747

5. Travascio, P., Li, Y., and Sen, D. (1998) Chem Biol 5, 505-517

6. Wilson, C., and Szostak, J. W. (1998) Chem Biol 5, 609-617

7. Brackett, D. M., and Dieckmann, T. (2006) Chembiochem 7, 839-843

8. Babendure, J. R., Adams, S. R., and Tsien, R. Y. (2003) J Am Chem Soc 125, 14716-14717

9. Grate, D., and Wilson, C. (1999) Proc Natl Acad Sci U S A 96, 6131-6136

10. Kleywegt, G. J. (1996) Acta Crystallogr D Biol Crystallogr 52, 842-857

11. Laskowski, R. A. (1995) J Mol Graph 13, 323-330, 307-328 
12. Fox, M., McIntyre, R., and Hayon, E. (1977) Faraday Discuss. Chem. Soc 64, 167-172

13. Guex, N., and Peitsch, M. C. (1997) Electrophoresis 18, 2714-2723

14. Nguyen, D. H., DeFina, S. C., Fink, W. H., and Dieckmann, T. (2002) J Am Chem Soc 124, $15081-15084$

15. Chen, D., and Laidler, K. (1959) Can. J. Chem. 37, 599-611

16. Cong, X., and Nilsen-Hamilton, M. (2005) Biochemistry 44, 7945-7954

17. Rusconi, C. P., Yeh, A., Lyerly, H. K., Lawson, J. H., and Sullenger, B. A. (2000) Thromb Haemost 84, 841-848

18. Flinders, J., DeFina, S. C., Brackett, D. M., Baugh, C., Wilson, C., and Dieckmann, T. (2004) Chembiochem 5, 62-72

19. Fukuda, K., Vishnuvardhan, D., Sekiya, S., Hwang, J., Kakiuchi, N., Taira, K., Shimotohno, K., Kumar, P. K., and Nishikawa, S. (2000) Eur J Biochem 267, 3685-3694

20. Kubik, M. F., Stephens, A. W., Schneider, D., Marlar, R. A., and Tasset, D. (1994) Nucleic Acids Res 22, 2619-2626

21. Gal, S. W., Amontov, S., Urvil, P. T., Vishnuvardhan, D., Nishikawa, F., Kumar, P. K., and Nishikawa, S. (1998) Eur J Biochem 252, 553-562

22. Tasset, D. M., Kubik, M. F., and Steiner, W. (1997) J Mol Biol 272, 688-698

23. Chen, H., McBroom, D. G., Zhu, Y. Q., Gold, L., and North, T. W. (1996) Biochemistry 35, 6923-6930

24. Chen, H., and Gold, L. (1994) Biochemistry 33, 8746-8756

25. Seiwert, S. D., Stines Nahreini, T., Aigner, S., Ahn, N. G., and Uhlenbeck, O. C. (2000) Chem Biol 7, 833-843

26. Kulbachinskiy, A., Feklistov, A., Krasheninnikov, I., Goldfarb, A., and Nikiforov, V. (2004) Eur J Biochem 271, 4921-4931 
27. Hwang, B., Cho, J. S., Yeo, H. J., Kim, J. H., Chung, K. M., Han, K., Jang, S. K., and Lee, S. W. (2004) Rna 10, 1277-1290

28. Allen, P., Worland, S., and Gold, L. (1995) Virology 209, 327-336

29. Schneider, D. J., Feigon, J., Hostomsky, Z., and Gold, L. (1995) Biochemistry 34, 9599-9610

30. Takeno, H., Yamamoto, S., Tanaka, T., Sakano, Y., and Kikuchi, Y. (1999) J Biochem (Tokyo) 125, 1115-1119

31. Thomas, M., Chedin, S., Carles, C., Riva, M., Famulok, M., and Sentenac, A. (1997) J Biol Chem 272, 27980-27986

32. Vo, N. V., Oh, J. W., and Lai, M. M. (2003) Virology 307, 301-316

33. Bell, S. D., Denu, J. M., Dixon, J. E., and Ellington, A. D. (1998) J Biol Chem 273, 1430914314

34. Gening, L. V., Klincheva, S. A., Reshetnjak, A., Grollman, A. P., and Miller, H. (2006) Nucleic Acids Res 34, 2579-2586

35. Smith, D., Kirschenheuter, G. P., Charlton, J., Guidot, D. M., and Repine, J. E. (1995) Chem Biol 2, 741-750

36. Kimoto, M., Shirouzu, M., Mizutani, S., Koide, H., Kaziro, Y., Hirao, I., and Yokoyama, S. (2002) Eur J Biochem 269, 697-704

\section{FOOTNOTES}

This work was supported by the Director, Office of Energy Science Research, Office Medical Sciences, Life Sciences of the U. S. Department of Energy under Contracts W-7405-Eng-82 with the Ames National Laboratories. The authors thank Mark Hargrove for advice on cavity assay, Amy Andreotti for advice on NMR, and Gaya Amarasinghe for help in interpreting the NMR data of the MGA variants. We also thank Bruce Fulton in the Biomolecular Structure Facility and Kamel 
Harrata and Shu $\mathrm{Xu}$ in the Chemical Instrumentation Facility at Iowa State University for their technical support in the NMR and mass spectrometry experiments.

\section{FIGURE LEGENDS}

Fig. 1 The $\mathrm{pH}$ dependence of $M G$ bleaching. A time course of MG bleaching was determined at each of a range of pHs using the buffers $16.7 \mathrm{mM}$ acetate, $\mathrm{pH} 5.0 ; 100 \mathrm{mM} \mathrm{KCl}, 5 \mathrm{mM} \mathrm{MgCl} 2,10 \mathrm{mM}$ HEPES, pH 7.2; 9.8 mM Tris, pH 9.0; $0.1 \mathrm{mM} \mathrm{NaOH,} \mathrm{pH} \mathrm{10;} 1 \mathrm{mM} \mathrm{NaOH}, \mathrm{pH} 11$ or $10 \mathrm{mM} \mathrm{NaOH}$, $\mathrm{pH}$ 12. From these time courses the rate constant of reaction $(\mathrm{k})$ and the half-lives $\left(\mathrm{t}_{1 / 2}\right)$ were determined and plotted against $\mathrm{pH} . \mathrm{k},(\Delta) ; \mathrm{t}_{1 / 2},(\mathrm{O})$. The insert shows a time course for the MG bleaching that can be fit to a curve for a second order reaction. This experiment was repeated three times for each $\mathrm{pH}$.

Fig 2. The chemistry of MG bleaching. (A, left) The mass spectrum by ESI of MG (molecular mass of $329 \mathrm{Da}$ ). (A, right) The mass spectrum of MG-OH by EI (molecular mass $346 \mathrm{Da}$ ). (B) The NMR spectra of MG (top) and MG-OH (bottom). (C) MG bleaching is proposed to proceed by an $\mathrm{OH}^{-}$ attack on the $\mathrm{C} 1$ of $\mathrm{MG}$, forming the carbinol base.

Fig. 3. The malachite green aptamer protects $M G$ from bleaching. A,B: The kinetics of MG bleaching. $2 \mu \mathrm{M}$ MG was incubated in $10 \mathrm{mM}$ HEPES, $100 \mathrm{mM} \mathrm{KCl}, 5 \mathrm{mM} \mathrm{MgCl}$, $\mathrm{pH}$ 7.2-7.4 in the absence $(\nabla)$ or presence of $(\diamond) 10 \mu \mathrm{M} 100 \mathrm{nt}$ random RNA or in the presence of various concentrations of the MGA: $0.5 \mu \mathrm{M}(\bullet), 1 \mu \mathrm{M}(\mathrm{x}), 2 \mu \mathrm{M}(*), 4 \mu \mathrm{M}(+), 6 \mu \mathrm{M}(\circ)$ MGA. C,D: The kinetics of MG bleaching in the presence of the MGA and varying concentrations of AS. $2 \mu \mathrm{M}$ MG was incubated for 2 min alone $(\diamond)$ or with $10 \mu \mathrm{M}$ MGA $(\Delta)$ in $10 \mathrm{mM}$ HEPES, $100 \mathrm{mM} \mathrm{KCl,} 5 \mathrm{mM} \mathrm{MgCl} 2$, pH 7.27.4. To this mixture was then added $500 \mu \mathrm{M}$ shuffled AS (*) or AS at concentrations of $20 \mu \mathrm{M}(\bullet)$ or $100 \mu \mathrm{M}(\mathrm{\circ})$ AS. A, C: The changes in MG color were tracked as a function of time. B,D: The constants $\mathrm{k}$ and $\mathrm{t}_{1 / 2}$ were determined from the plots in $\mathrm{A}$ and $\mathrm{C}$. The figure shows the results of one of three independent experiments with consistent results. 
Fig. 4. Cavity analysis by Surfnet of MG alone and in the MGA binding pocket. A) MG (magenta) is flanked in the binding pocket of MGA (1q8n.pdb) by C7, G24, G29, and A31 (orange) on one side and G8 and C28 (yellow) on the other. Stem I (red), the base triples (green), Stem II (blue), and the Tetraloop (purple) are also shown. B) MG contains three aromatic rings around the central carbon (green). Hydroxide molecules can attack from above or below the plane, but C) the rings block approach within the plane of the ring. D) Stereoview of the MG binding pocket of MGA, colored as above, with cyan mesh indicating the area accessible to an hydroxide of minimum radius. The space above and below the plane of MG is blocked by the bases of the binding pocket and is therefore not accessible, as indicated by the lack of mesh. The foreground of the image has been slabbed away to assist in viewing the interior of the binding pocket.

Fig. 5. Mutations around the MGA binding pocket compromise its ability to protect MG. MG bleaching at the presence of the MGA variants at concentrations equivalent to 10-15 times $\mathrm{K}_{\mathrm{D}}$ was measured by spectrophotometry. The concentrations of interacting components were: $\odot: 3$ or $30 \mu \mathrm{M}$ MG; $\square: 30 \mu \mathrm{M}$ MG, 3 mM tRNA; $: 3 \mu \mathrm{M}$ MG, $10 \mu \mathrm{M}$ MGA; ०: $3 \mu \mathrm{M}$ MG, 20-30 $\mu \mathrm{M}$ MGA(A31C); $\diamond: 30 \mu \mathrm{M}$ MG, 140-200 $\mu \mathrm{M}$ MGA(U25C); $\Delta: 30 \mu \mathrm{M}$ MG, 3 mM MGA(G8C, G24C, G29C). The figure shows the results, for the MGA and each MGA variant, of one of two to four independent experiments were performed that all gave consistent results.

Fig. 6. Footprinting analysis of the binding pockets of the MGA variants. A. RNase I footprinting of the MGA with and without increasing MG concentrations (0.05 to $4 \mu \mathrm{M})$. B. RNase I footprinting of the MGA(A31C) with and without $20 \mu \mathrm{M}$ MG which was equivalent to 10 times $K_{D}$. C. RNase I footprinting of the MGA(U25C) with and without $140 \mu \mathrm{M}$ MG which was equivalent to 10 times $\mathrm{K}_{\mathrm{D}}$. D. RNase I footprinting of the MGA(G8C-G24C-G29C) with and without $3 \mathrm{mM}$ MG that was equivalent to 10 times $\mathrm{K}_{\mathrm{D}}$. Each experiment was repeated as least twice. 
Fig. 7. ${ }^{1} H$-NMR spectra showed proper folding of the $M G A$ variants which could bind $M G$. The ${ }^{1} \mathrm{H}-$ NMR spectra includes two regions: 11-15 ppm (A) and 6.5-9.0 ppm. The samples were $100 \mu \mathrm{M}$ MG, $50 \mu \mathrm{M}$ MGA $\pm 100 \mu \mathrm{M}$ MG, $50 \mu \mathrm{M}$ MGA(U25C) $\pm 200 \mu \mathrm{M}$ MG, $50 \mu \mathrm{M}$ MGA(A31C) $\pm 100 \mu \mathrm{M}$ $\mathrm{MG}$, and $50 \mu \mathrm{M}$ MGA(G8C-G24C-G29C) $\pm 500 \mu \mathrm{M}$ MG. 


\section{TABLES}

Table I. Affinities and $\lambda \max$ of the interaction of MG with the MGA and its variants.

\begin{tabular}{|l|l|l|l|l|l|}
\hline \multicolumn{2}{|c|}{ RNA } & MG & Kd $(\mu \mathrm{M})$ & \multicolumn{1}{c|}{$\lambda$ max } & $\mathrm{t}_{1 / 2}$ of MG \\
bleaching \\
MGA & & & & \\
MGA(A31C) & $(0.1-15 \mu \mathrm{M})$ & $0.2 \mu \mathrm{M})$ & $1.03 \pm 0.53$ & 632 & $802 \pm 611 \mathrm{~h}$ \\
MGA(U25C) & $(0.1-15 \mu \mathrm{M})$ & $0.2 \mu \mathrm{M}$ & $1.93 \pm 0.97$ & 632 & $21.8 \pm 16.2 \mathrm{~h}$ \\
MGA(G8C-G24C-G29C) & $(0.025-1.7 \mathrm{mM})$ & $20 \mu \mathrm{M}$ & $274 \pm 65$ & 629 & $4.76 \pm 2.78 \mathrm{~h}$ \\
AS & $(1-400 \mu \mathrm{M})$ & $0.4,2 \mu \mathrm{M}$ & $13.8 \pm 3.40$ & 629 & $20.9 \pm 11.5 \mathrm{~h}$ \\
tRNA & $(0.025-1.2 \mathrm{mM})$ & $20 \mu \mathrm{M}$ & $172 \pm 13$ & 629 & not determined \\
- & $(0.020-3 \mathrm{mM})$ & $20 \mu \mathrm{M}$ & $210 \pm 41$ & 629 & $7.38 \pm 0.04 \mathrm{~h}$ \\
& & $2,20 \mu \mathrm{M}$ & & 618 & $0.70 \pm 0.07 \mathrm{~h}$ \\
\hline
\end{tabular}

The MGA and its variants, tRNA and AS were tested for their abilities to bind MG. The $\mathrm{K}_{\mathrm{D}}$ 's were determined using the concentrations of MG and RNAs shown in the table. The incubations were performed in $100 \mathrm{mM}$ Tris, $100 \mathrm{mM} \mathrm{KCl,} 5 \mathrm{mM} \mathrm{MgCl} 2$, $\mathrm{pH}$ 9.0. Each titration included at least 6 different concentrations of the RNA. The $t_{1 / 2}$ of MG bleaching was measured in the presence of concentrations equivalent to $10-15 \mathrm{xK}_{\mathrm{D}}$ of the MGA variants or tRNA. The data for $\mathrm{t}_{1 / 2}$ are the averages of three independent experiments shown with the standard deviation of the average. 
FIGURES

Fig. 1

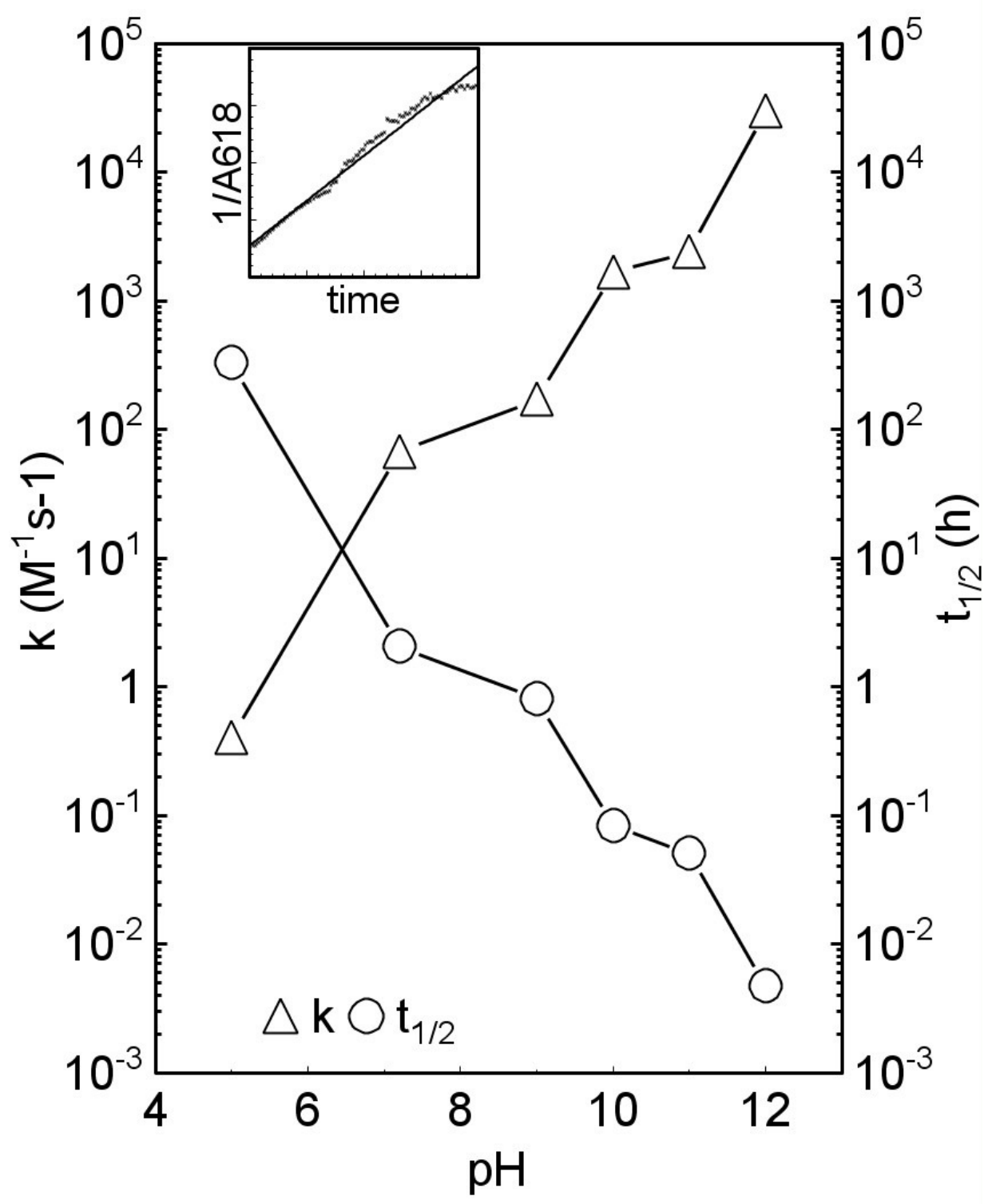


Fig. 2

A

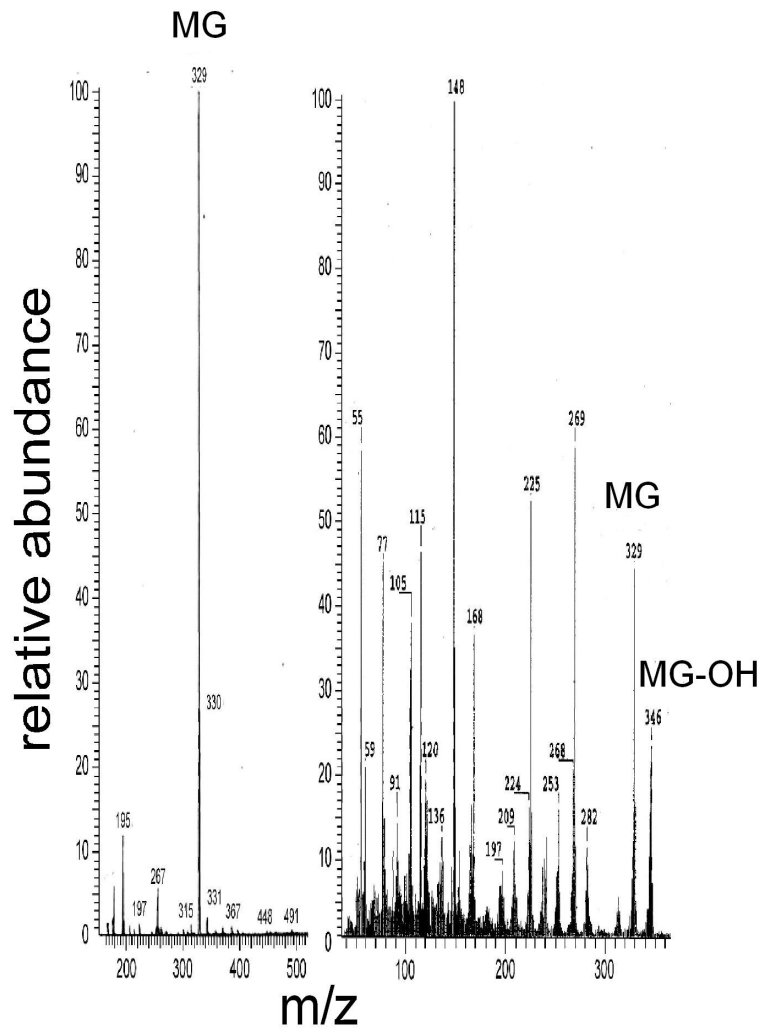

B
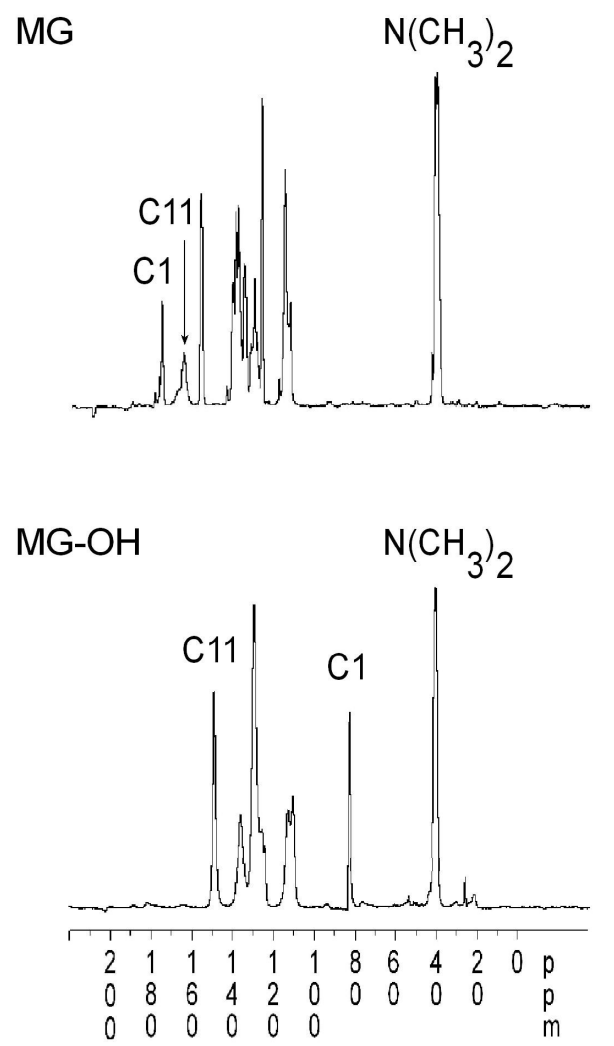

C<smiles>COOCC(c1ccccc1)(c1ccc(N(C)C)cc1)C1C=CC(=[N+](C)C)C=C1</smiles> 
Fig. 3

A

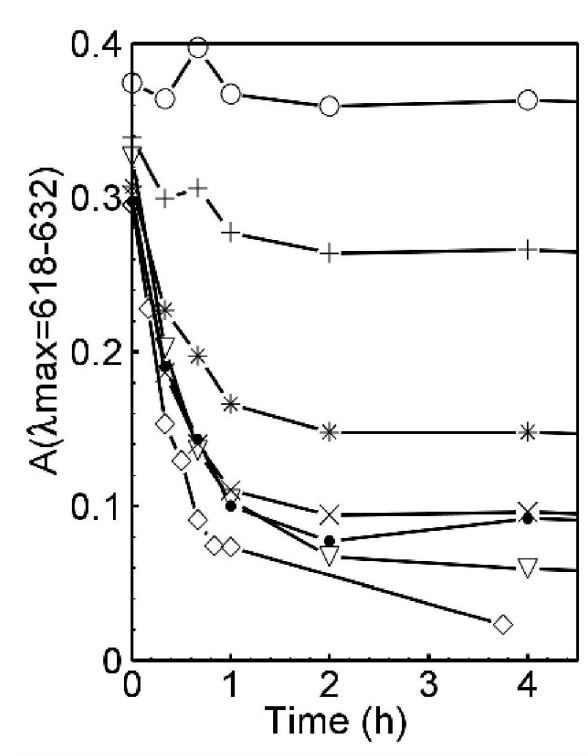

C

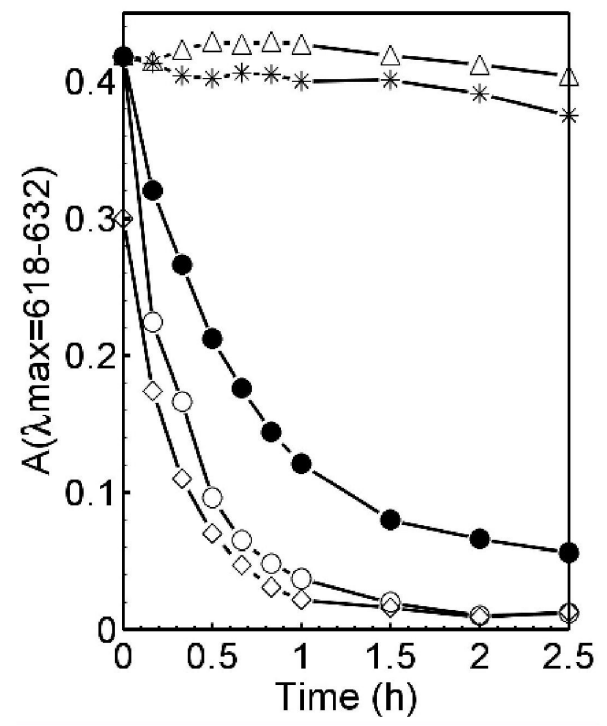

B

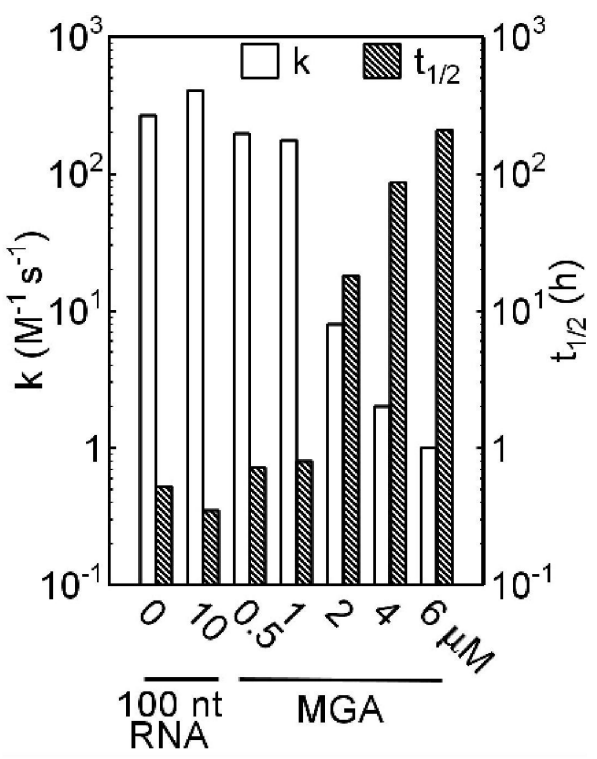

D

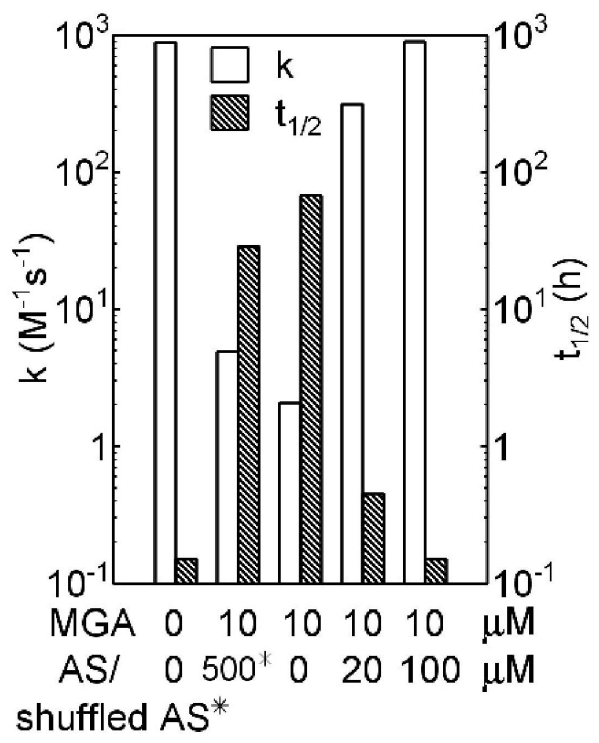


Fig. 4
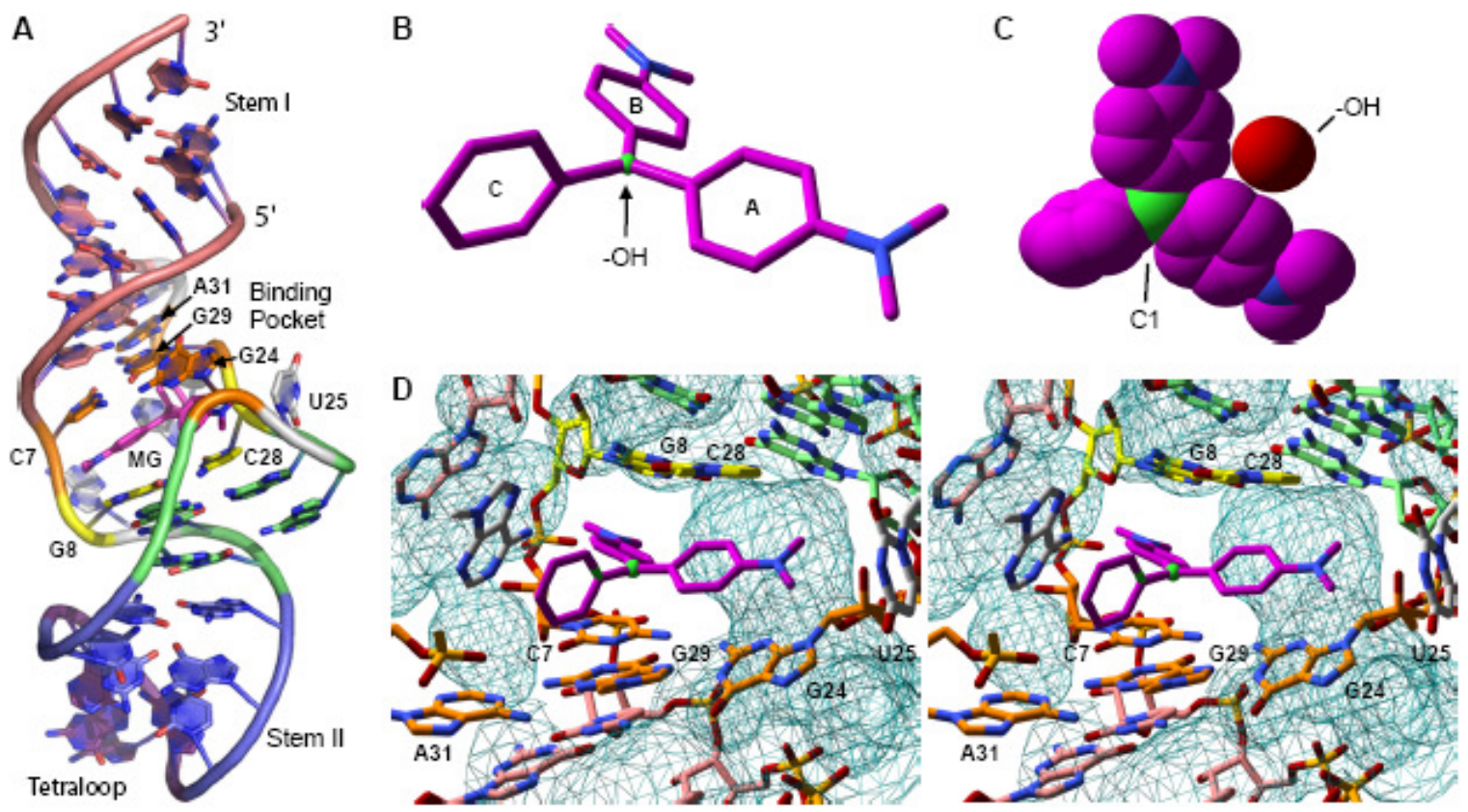
Fig. 5

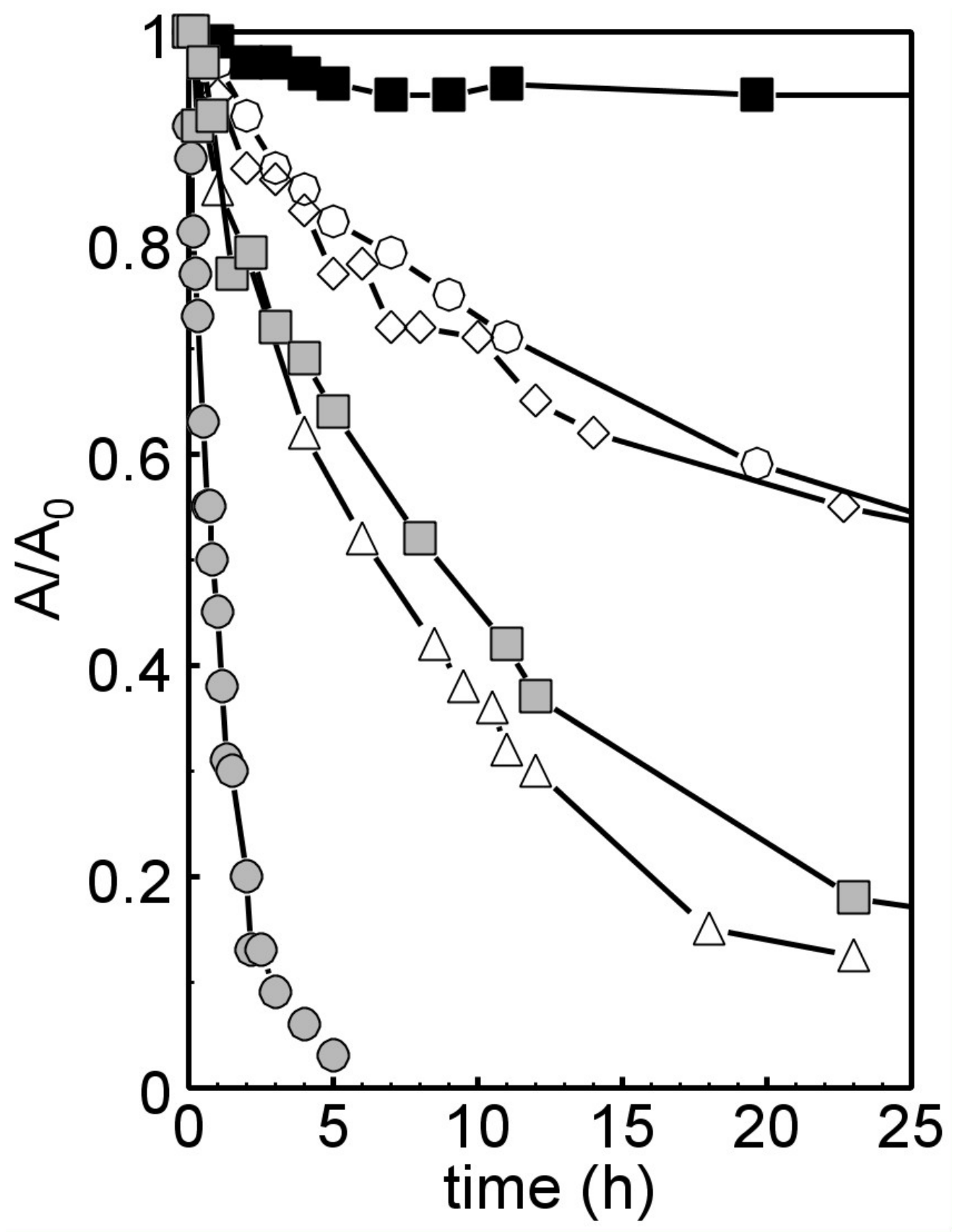


Fig. 6
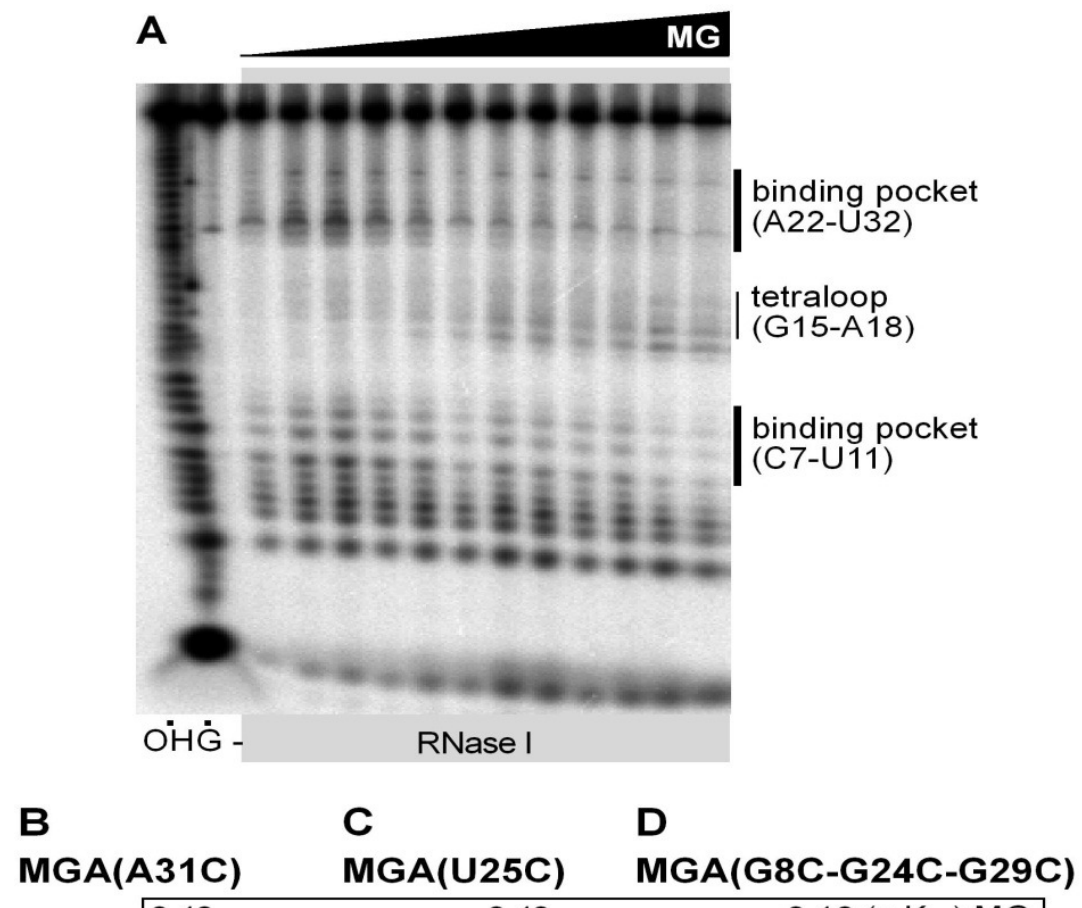

\begin{tabular}{lll}
010 & 010 & $010\left(x K_{D}\right) M G$ \\
\hline
\end{tabular}

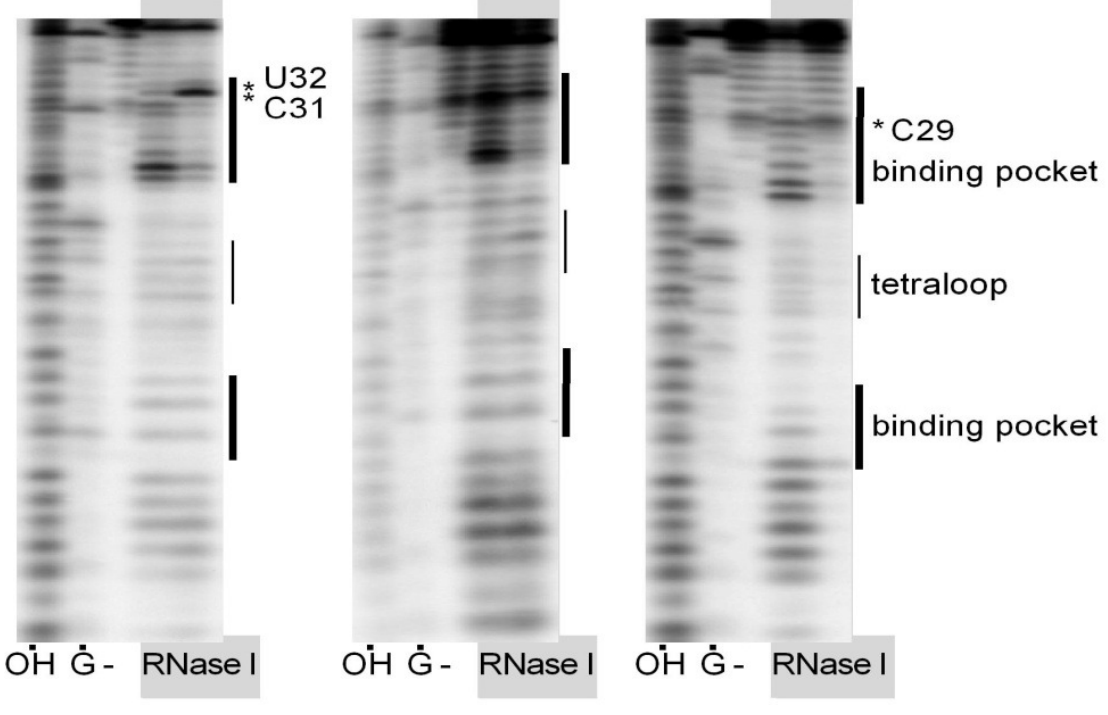


Fig. 7

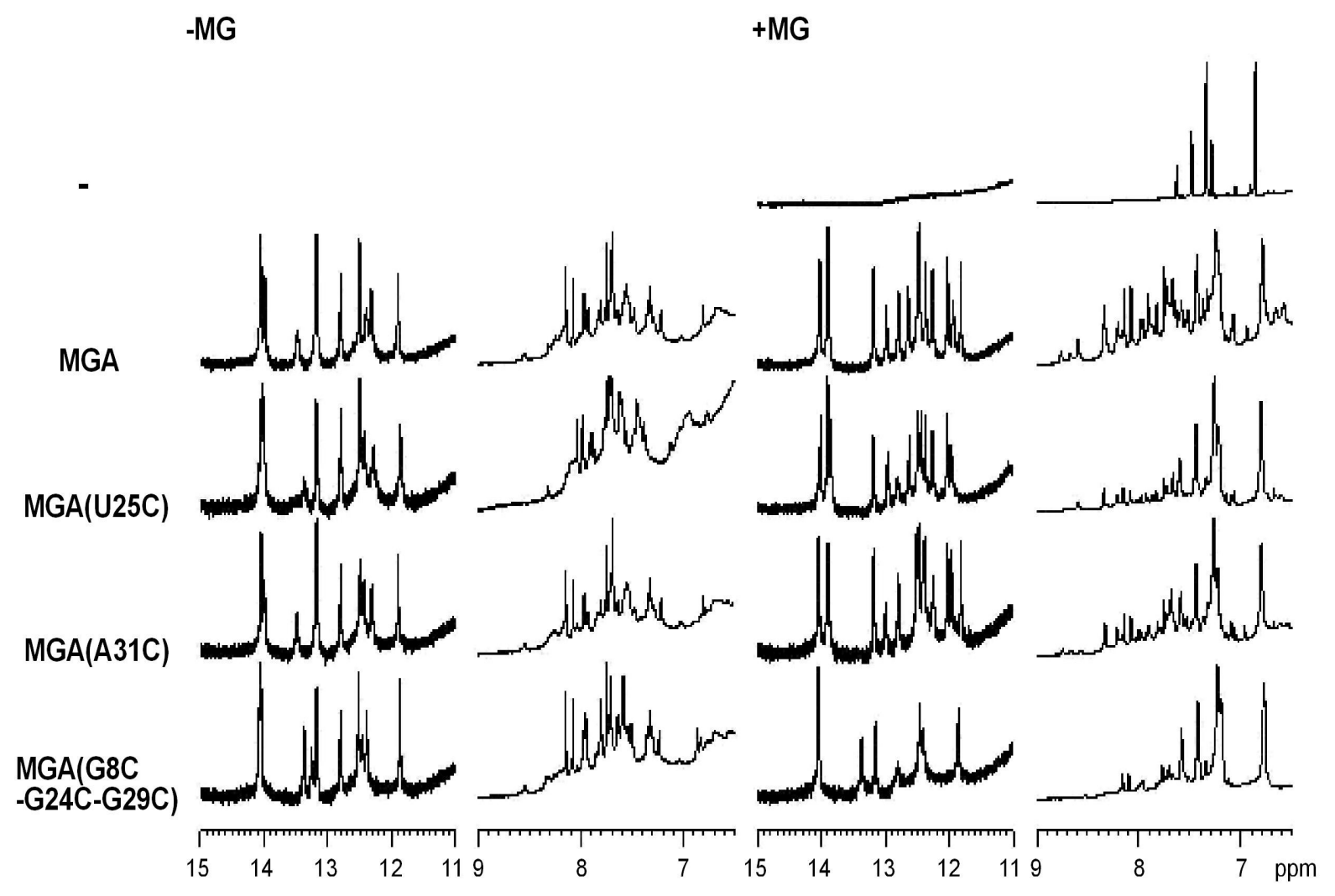




\title{
CHAPTER 4. TWO-STEP MECHANISM OF MALACHITE GREEN - MALACHITE GREEN APTAMER RECOGNITION
}

\author{
Tianjiao Wang ${ }^{1}$, Monica Lamm², Julie Hoy ${ }^{1}$, and Marit Nilsen-Hamilton ${ }^{1}$ \\ ${ }^{1}$ Department of Biochemistry, Biophysics and Molecular Biology and ${ }^{2}$ Department of Chemical \\ and Biological Engineering, Iowa State University, Ames, IA 50011 \\ running title: Two Step Mechanism of MG-MGA interaction
} Corresponding author: M.N.H. E-mail address: $\underline{\text { marit@iastate.edu }}$

\begin{abstract}
Abbreviations used: malachite green (MG); tetramethylrosamine (TMR); malachite green aptamer (MGA); molecular dynamics simulation (MD simulation); Isothermal titration calorimetry (ITC); nuclear magnetic resonance spectrometry ${ }^{1}$.
\end{abstract}




\begin{abstract}
Experimental structural analysis by molecular biophysical methods provides atom-level resolution that is the basis for understanding molecular function. However, due to limitations in the experimental technology, the dynamics must often be inferred from static structures. Computational methods have been developed to fill this void, but have often not been rigorously tested against the experimental data. Here we explore the interaction between the malachite green aptamer (MGA) and its target molecule, malachite green (MG) by using molecular dynamics (MD) simulation with experimental confirmation. MD simulation predicted that the MG binding pocket of the MGA is largely pre-organized and that binding of MG involves reorganization of the pocket and a simultaneous twisting of the MGA terminal stem around the pocket. These predictions were confirmed by analysis of the MGA-MG interaction by RNase I footprinting, melting profiles, 1D NMR, isothermal titration calorimetry, and stopped-flow kinetics. Thus, by using molecular dynamics modeling to extend the structural information derived by NMR and crystallography we were able to predict an experimentally verifiable dynamic model of the unoccupied MGA that has not yet been obtained by biophysical analysis. The strategy used here may serve as a general approach to obtain a realistic simulation of the 3D-structure of an unoccupied aptamer starting with the experimentally determined structure of the aptamer-target complex, which then allows dynamical studies of the interaction of aptamer and its target.

Keywords: thermodynamics, kinetics, molecular dynamics simulation, malachite green, aptamer
\end{abstract}




\section{INTRODUCTION}

X-ray crystallography and NMR are powerful tools for establishing molecular structure with atomic resolution. However, there are many instances in which the use of these technologies is not possible due to the limitations of the methods or lack of adequate samples. Although these methods, particularly NMR, can provide information about molecular dynamics, this information cannot always be obtained under physiological conditions or under the same conditions as the protein or nucleic acid is studied for its function in vitro. Computational simulations are commonly used to provide further insight to molecular structure and function when the current limitations of biophysical applications are reached. However, few simulations are rigorously tested experimentally and thus the value of this approach is still uncertain.

Aptamers are single stranded nucleic acids that bind to their targets with high specificities and affinities ( $\mathrm{K}_{\mathrm{D}} \mathrm{S}$ in the $\mathrm{pM}$ to $\mu \mathrm{M}$ range) that approach those of antibodies. Although there are a few tertiary structures of unoccupied aptamers (thrombin aptamer ${ }^{1}$, nucleolin aptamer ${ }^{2}$ and NFkB aptamer ${ }^{3}$ ), more than 40 tertiary structures of aptamer-target complexes have been solved by X-ray crystallography or by $\mathrm{NMR}^{4 ; 5}$ and have been deposited in a protein data bank (www.pdb.org) ${ }^{6}$. These 3D structures provide static views of the physical interactions between aptamers and their targets but only NMR reveals information about the dynamics of the aptamer-target interaction. However, good NMR data cannot always be obtained under conditions equivalent to those in vivo or as the molecules are studied in vitro. Knowledge of the dynamics of aptamer structures are important because it provides opportunities to develop regulated aptamers that can be used for many useful sensor applications.

Various experimental approaches have been used to study aptamer dynamics, including single molecule force measurements ${ }^{7 ; 8}$, fluorescence resonance energy transfer ${ }^{9 ; 10}$, NMR ${ }^{11 ; 12 ; 13 ; 14 ;}$ ${ }^{15 ; 16}$, stopped-flow kinetics ${ }^{17 ; 18 ; 19}$, isothermal titration calorimetry (ITC) ${ }^{17 ; 20}$ and chemical and 
enzymatic structure probing ${ }^{10 ; 21}$. Aptamer-target interactions can involve multiple steps ${ }^{7 ; 10 ; 15 ; 16 ; 17 ;}$ ${ }^{18 ; 19}$ and binding can be enthalpic ${ }^{17 ; 20 ; 22}$ or entropic $^{23}$. Aptamers differ greatly in their kinetic parameters with measured association rates varying between $10^{3}$ to $10^{9} \mathrm{M}^{-1} \mathrm{~s}^{-1}$, dissociation rates of $10^{-6}$ to $10^{2} \mathrm{~s}^{-1}$ or dissociation half lives of $10^{-3} \mathrm{~s}$ to $50 \mathrm{~h}$ reported for different aptamers, with the thrombin ${ }^{24}$ and CD30 aptamers ${ }^{25}$ being two extreme examples. According to dynamical and structural studies, three different models of aptamer-target interaction have been proposed: adaptive recognition (induced fit) ${ }^{4}$, preformed (lock and key) ${ }^{21}$, and a combination of the two ${ }^{3 ; 10 ; 13 ; 14}$. Molecular dynamic (MD) simulations have been applied to understand the dynamics of aptamertarget interactions for aptamers that recognize FMN ${ }^{26 ; 27}$, theophylline ${ }^{28 ; 29 ; 30}$, L-argininamide ${ }^{22}$, paromomycin ${ }^{31}$, thrombin ${ }^{32}$, glutaminyl-tRNA synthetase ${ }^{23}$ and the HIV transactivation response element ${ }^{33}$. These studies examined the entropic and enthalpic contributions to binding to develop structural models of aptamer-target interactions. In these studies, the free energies of binding differed between the simulated and experimental structures by $7-16 \%$ while the root mean square deviations (RMSD) differed by 2-4 A. The results of these studies suggest that MD simulation could be an effective means of predicting the dynamic nature of aptamer-target interactions based on initial biophysical data.

To examine the ability of MD simulations to predict the dynamical aspects of aptamer-target interactions that are not available from biophysical analysis, we chose to study the malachite green aptamer (MGA), which is a 38 nt RNA aptamer consisting of stem I (residues 1-6, 33-38), a binding pocket (residues 7-11 and 22-32), stem II (residues 12-14, 19-21) and a tetraloop (residues 15-18) (Figs. 1,8) ${ }^{34 ; 35}$. We show here that MD simulation of the MGA in the presence and absence of its malachite green (MG) target predicted a dynamical structure of the unoccupied MG that has not been solved experimentally. The simulated structure was shown to be consistent with biochemical probing of the MGA and the MGA-MG complex. The results of molecular simulations combined with experimental approaches that included thermodynamic, kinetic and structural probing suggest a two- 
step kinetic model for the interaction of the MGA with MG. Our studies demonstrate the application of MD simulations to extending the results of biophysical measurements to understand the mechanism and dynamics of molecular interactions.

\section{RESULTS}

\section{MD simulation of MGA with and without bound MG}

Both NMR and crystal structures have been solved for the MGA in complex with MG or $\mathrm{TMR}^{34 ; 35}$. However, no structure has been solved for the unoccupied MGA, leaving an important gap in our knowledge of the structure of this aptamer that limits our understanding of the molecular event involved in the process of aptamer-target interactions. To address this problem, we used MD simulation to predict the structure of the unoccupied aptamer. Model 1 of the 25 MGA-MG NMR structures (PDB ID: 1Q8N) was used as the input.

To simulate the unoccupied MGA, the coordinates of the MGA-MG complex were used with the MG removed. The MGA and MGA-MG complex were both solvated with water and $\mathrm{Mg}^{2+}$ and simulated over 7.7 and $7.0 \mathrm{~ns}$ respectively. The simulations reached equilibrium after $0.5 \mathrm{~ns}$ as judged by constant temperature and no more increase in RMSD compared with the starting structure. The molecular coordinates were then allowed to evolve over 7.2 and $6.5 \mathrm{~ns}$ respectively. The two equilibrated structures were compared in four structural regions: 1) stem I (residues 1-6, 33-38), 2) binding pocket (residues 7-11, 22-32), 3) stem II (residues 12-14, 19-21) and 4) the tetraloop (residues 15-18) (Fig. 1).

The simulations predicted that stem I of both the MGA and MGA-MG structures are unstable with dynamic formation and disruption of $\mathrm{H}$-bonds over the simulation period. MG binding stabilized the A3-U36 base pairing while it destabilized the G2-C37 (data not shown) and U4-A35 base pairing (Fig. 1 (a)). MG binding also switched the C6-G33 base-pairing from strong H-bonding (N4-H-O6, N3-H-N1 and O2-H-N1) to weaker H-bonding (O2-H-N1) (data not shown). These large 
fluctuations in stem structure were not evident in the NMR pdb dataset. By contrast with stem I, the simulated H-bond structure and stability of stem II was similar to that observed by NMR and was not predicted to be altered by formation of the MGA-MG complex (data not shown). Alignment of the average simulated structure of MGA-MG complex and the average simulated structure of MGA showed that stem I of the MGA-MG was predicted to be altered relative to its position in the MGA (Fig. 1 (a)).

In the MGA binding pocket MG is stacked between two sets of hydrogen-bonded bases, a base quadruple C7-G29-G24-A31 on top and a G8-C28 base-pair underneath. In the simulated unoccupied MGA, the base quadruple was absent. In its place was observed a base triple (G24-G29A31) with dynamic G24-G29 base pairing and no H-bonding between C7 and G29. Thus, the MD simulation predicts that formation of the base quadruple accompanies the binding of MG. By contrast the G8-C28 base-pair that forms the lower stacking platform for MG was predicted to be present in the unoccupied MGA and this base-pairing was stabilized in the MG-MGA structure. Two other base triples $\mathrm{C} 10-\mathrm{G} 23-\mathrm{A} 27$ and U11-A22-A26 that are also present in the binding pocket are predicted to preexist in MGA and neither are predicted to be altered upon MG binding. The C10-G23-A27 base triple was simulated as very stable while the U11-A22-A26 base triple was very dynamic (data not shown).

Base-stacking of the tetraloop was monitored in the simulation by the distance between the $\mathrm{C} 5$ atom of residue A16 and the $\mathrm{C} 4$ atom of residue G17. The simulation showed dynamic stacking and unstacking between A16 and G17 in the tetraloop that was destabilized in the MGA-MG complex. The relative position and orientation of tetraloop also changed between the average simulated structures of MGA and the MGA-MG structure (Fig. 1(b)).

In addition to the simulation of MGA and MGA-MG solvated with $\mathrm{Mg}^{2+}$, MGA-MG solvated with $\mathrm{K}^{+}$was also carried out. After $0.5 \mathrm{~ns}$ simulation, the system reached equilibrium and then the coordinate was allowed to evolve for another $2.5 \mathrm{~ns}$. Consistent with the simulation result of MGA- 
MG solvated with $\mathrm{Mg}^{2+}$, the simulation of MGA-MG solvated with $\mathrm{K}^{+}$also showed dynamic openclose stem I, a binding pocket consisting of dynamic base quadruple (G24-C7-G29-A31), a stable base-pair (G8-C28), two dynamic base triples (C10-G23-A27 and U11-A22-A26), a stable stem II and a dynamic tetraloop. The simulation of MGA-MG solvated with $\mathrm{Mg}^{2+}$ and $\mathrm{K}^{+}$also showed structure difference as shown by the RMSD $(2.302 \AA$ for the whole molecule and $1.889 \AA$ for the MG binding pocket of MGA) (Table 3). The main simulated structure difference between MGA-MG solvated $\mathrm{Mg}^{2+}$ and $\mathrm{K}^{+}$existed in stem I and binding pocket. There were dynamic formation and disruption of H-bonds in G2-C37 and U4-A35 at the presence of $\mathrm{Mg}^{2+}$ but in C5-G34 and C6-G33 at the presence of $\mathrm{K}^{+}$. In addition, $\mathrm{H}$-bond formed in A3-U36 at the presence of $\mathrm{Mg}^{2+}$ but didn't exist at the presence of $\mathrm{K}^{+}$. In the base quadruple of the MG binding pocket of MGA-MG complex, H-bonds formation was between C7-G29, G29-G24 and G29-A31 at the presence of $\mathrm{Mg}^{2+}$ but was between C7-G29, C7-G24 and G29-A31 at the presence of $\mathrm{K}^{+}$(data not shown). To sum, the simulation of MGA-MG at the presence of $\mathrm{Mg}^{2+}$ and $\mathrm{K}^{+}$gave consistent overall structure with some difference in stem I and binding pocket.

Thus, the simulation predicted that stem I of the MGA-MG complex is more unstable than observed by NMR, that the binding pocket of the unoccupied MGA is partially formed and that the stem I and tetraloop structure is altered on MG binding.

\section{RNase I footprinting confirms predicted MGA structures}

RNase I footprinting of MGA in the presence and absence of MG was performed to test the predictions of the MD simulations. RNase I cleaves any residues in single-stranded regions but not double stranded regions of RNA. The results showed strong cleavage of C7, G24 and U25 and weak or negligible cleavage of other residues in the MGA binding pocket of the unoccupied MGA. The aptamer was protected against cleavage by RNase I in these regions upon binding MG (Fig. 2). These results are consistent with the predicted preorganized binding pocket and induced fit mechanism of 
MG binding. MG binding to MGA also resulted in decreased cleavage by RNase I of stem I and slightly increased cleavage of the tetraloop. These results are consistent with the predicted global conformational change of MGA upon MG binding. Thus, the RNase footprinting results appeared more consistent with the simulated structure than the NMR-derived structure of the MGA-MG complex.

\section{${ }^{1}$ H-NMR spectra show buffer effects on the MGA structure}

The NMR-derived structure and the RNase I structural probing of the MG-MGA complex were performed in buffers that differed in $\mathrm{pH}$ and concentrations of $\mathrm{MgCl}_{2}$ and $\mathrm{KCl}$. To determine if the MGA structure is sensitive to these differences, we examined the effects of these buffer conditions on the MGA conformation in the presence and absence of MG using ${ }^{1} \mathrm{H}-\mathrm{NMR}$ spectroscopy (Fig. 3). The spectra showed that under all conditions the number of peaks in the 10-15 ppm region, from the imino $\mathrm{NH}(\mathrm{H} 1,3)$ resonance $(\mathrm{N}-\mathrm{H} . . . \mathrm{N})$ that are involved in base-pairing, increased in the presence of MG. Also, under all conditions when MGA was present a broadening effect was observed on the MG peaks in the range 6.8-7.6 ppm that includes resonance of amino $\mathrm{NH}_{2}$ and $\mathrm{CH}(\mathrm{H} 2,6,8)$ on the benzyl rings. These results demonstrate that MG was bound by MGA under

all conditions tested. However, the spectral differences for MGA and the MGA-MG complex showed that $\mathrm{pH}, \mathrm{MgCl}_{2}$ and $\mathrm{KCl}$ concentrations all affect the structures of the MGA and the MGA-MG complex. Thus, the difference between the NMR-derived structure and the RNase I-probed structure could be due to the differences in the buffers used for these analyses.

\section{Melting profiles (Tm) are consistent with simulation predictions}

To further examine the possibility that the discrepancy between the NMR-derived data and results from the RNase I footprinting assays might be due to differences in buffer conditions, we examined the melting profiles of MGA with and without MG in different buffer conditions and determined the Tm values (Table 1, Fig 4). Depending on the $\mathrm{pH}$ and salt constituents, the Tm value 
of the MGA-MG complex was 6-14 ${ }^{\circ} \mathrm{C}$ higher than that of unoccupied MGA, indicating reorganization to a more stable structure as a result of MG binding. Changing the $\mathrm{pH}$ from 5.8 to 7.2 and changing the concentration of $\mathrm{KCl}$ from $10 \mathrm{mM}$ to $100 \mathrm{mM}$ decreased the Tm of MGA with and without $\mathrm{MG}$ by $\sim 7^{\circ} \mathrm{C}$ and $\sim 3^{\circ} \mathrm{C}$ respectively while $5 \mathrm{mM} \mathrm{MgCl}_{2}$ increased the Tm value of the MGAMG complex by $3^{\circ} \mathrm{C}$. These data suggest that the buffer $\mathrm{pH}$ and salt constituents alter the structure of the MGA and MGA-MG complex, which may explain why the RNase footprinting results are inconsistent with the NMR-derived structure.

The melting profiles of MGA-MG complex in buffer N (NMR conditions) and buffer F (footprinting conditions) differed dramatically with the latter showing two transitions. The first transition is consistent with an increase in base-pairing with increased temperature in the $40^{\circ} \mathrm{C}$ range, while the second transition is consistent with melting of the molecular structure. The lower temperature transition may reflect more flexibility in the molecule at higher temperatures allowing the bases in a strained stem I to more readily base pair. These results are consistent with the predictions of the MD simulations.

\section{Footprinting of tandemly-linked MGAs confirms the MD-predicted twisting}

A prediction of the MD simulation is that MG binding by MGA is accompanied by formation of the C7-G29-G24-A31 base quadruple (Fig. 1) and switching of the C6-G33 base pair from three Hbonds (N4-H-O6, N3-H-N1 and O2-H-N1) to one H-bond (O2-H-N1) (data not shown). Alignment of the average simulated structure of the equilibrated MGA and MGA-MG either on the whole MGA or just in the region containing the binding pocket showed that the predicted MGA-MG and MGA structures deviated in the stem I and tetraloop regions (Fig. 1(b)). Thus, the simulation predicted that, with MG binding, the MGA twists along the vertical axis of the binding pocket and stem II resulting in a global conformational change of MGA in the regions of stem I and the tetraloop. 
To test the predicted twisting of MGA on MG binding, we prepared an RNA molecule (4MGA) consisting of 4 tandemly linked MGAs with a shorter linker of 7 bases (UAAAAAC) between each MGA unit. If the prediction is correct, the twisting force generated by the MGA-MG interaction should change the structure of the linkers making them more or less available to RNase I digestion. RNase I footprinting of 4MGA showed that, as for MGA, the binding pocket of each MGA unit was protected from RNase I cleavage upon MG binding. The data also showed that, as predicted from the MD simulation, increasing the MG concentration resulted in increasing cleavage of the linker regions (Fig. 5). This result is consistent with the twisting motion predicted by the simulation to occur in the MGA upon MG binding.

\section{Thermodynamic parameters are consistent with a structural rearrangement of the MGA upon MG binding}

Isothermal titration calorimetry was used to determine the thermodynamic parameters of the MG-MGA interaction (Fig. 6, Table 2). Although decolorization of MG (due to hydroxylation of the $\mathrm{C} 1$ carbon) occurred slowly during the titration in buffer at $\mathrm{pH} 7.0$, no significant heat release was attributable to decolorization. This was demonstrated by a blank titration under the same experimental condition (data not shown). The calculated $\mathrm{K}_{\mathrm{D}}$ for the MGA-MG interaction was $0.33 \pm 0.09 \mu \mathrm{M}$ and was similar to that measured by RNase I probing $(0.26-1.86 \mu \mathrm{M})$, stopped-flow kinetics $(0.20 \mu \mathrm{M})$ and fluorescence spectrophotometry $(0.6 \mu \mathrm{M}$, data not shown). The ITC analysis showed that the MGA-MG interaction had a favorable enthalpy $(\Delta \mathrm{H}=-24.7 \pm 0.97 \mathrm{kcal} / \mathrm{mol})$ and unfavorable entropy $(\Delta \mathrm{S}=-53.2 \pm 2.6 \mathrm{kcal} / \mathrm{mol} / \mathrm{deg})$, which is consistent with a possible structural rearrangement during MG binding. TMR differed from MG by an additional oxygen bridging two benzyl rings, which makes TMR flatter than MG. TMR bind to MGA with even higher affinity $\left(\mathrm{K}_{\mathrm{D}}=95 \mathrm{nM}\right)$. Unlike $\mathrm{MG}$, TMR is stable under the buffer condition. The thermodynamics of MGA-TMR interaction $(\Delta \mathrm{H}=-$ 
$15.3 \pm 0.60 \mathrm{kcal} / \mathrm{mol}, \Delta \mathrm{S}=-19.1 \pm 2.5 \mathrm{kcal} / \mathrm{mol} / \mathrm{deg})($ Table 2$)$ also indicated a structural rearrangement during MGA-target interaction.

\section{Kinetic parameters are consistent with structural rearrangements during MG binding}

Stopped-flow kinetics was used to measure the association rate $\left(\mathrm{k}_{\mathrm{on}}\right)$ and dissociation rate $\left(\mathrm{k}_{\mathrm{off}}\right)$ of the MG-MGA interaction (Fig. 7). From these studies we determined $\mathrm{k}_{\text {on }}$ as $2.32 \pm 0.76 \times 10^{-6} \mathrm{M}^{-}$ ${ }^{1} \mathrm{~s}^{-1}, \mathrm{k}_{\text {off }}$ as $0.39 \pm 0.13 \mathrm{~s}^{-1}$ and $\mathrm{K}_{\mathrm{D}}$ as $0.2 \pm 0.02 \mu \mathrm{M}$. The measured $\mathrm{k}_{\text {on }}$ of the MGA-MG interaction is

$\sim 1000$ fold slower than the diffusion limit $\left(\sim 10^{9} \mathrm{M}^{-1} \mathrm{~s}^{-1}\right)$. This time delay in MG binding is consistent with the predicted structural rearrangement of MGA during formation of the MG-MGA complex.

\section{DISCUSSION}

\section{Predictions of the MD simulation compared with experimental data}

Previous MD simulations of aptamers have resulted in simulations that differed by $2-4 \AA$ (RMSD) from the experimental structures $22 ; 23 ; 26 ; 27 ; 28 ; 29 ; 30 ; 31 ; 32 ; 33$. Our simulated average structure of the MGA-MG complex differed from the average NMR structure with an RMSD of $3.463 \AA$ for the whole complex and an RMSD $2.604 \AA$ for the binding pocket only. Even the two experimental determined 3D structures of MGA-MG ${ }^{34}$ and MGA-TMR ${ }^{35}$ complex differed by an RMSD of 4.070 $\AA$ for the whole molecules and an RMSD of $2.698 \AA$ for the binding pocket (Table 3 ). The likely reasons for the differences for these experimentally-determined structures are different $\mathrm{pH}$ and salt conditions in each determination and that TMR differs from MG by an additional oxygen atom bridging two benzyl rings to make TMR a flatter molecule than MG.

There are some discrepancies among the structures determined by MD simulation, RNase I footprinting, NMR spectrometry and crystallography. These discrepancies were in the stem I and in the binding pocket. The simulation showed a dynamic open-close stem and this mobility was verified by RNase I probing. However, this dynamic feature of stem I was not observed in the 3D NMR 
structure MGA-MG and the crystal structure of MGA-TMR complex. The binding pocket of MGAMG complex had $\sim 2.7 \AA$ difference in RMSD between the MD simulation and the NMR-determined structure (Table 3). The base-pairing in the binding pocket of the MGA differed between the experimental and simulated structures with the experimental determination showing only U11-A22 base pairing ${ }^{34}$ whereas the simulated structures predicted base-pairing of G8-C28, C7-G29-A31, C10-G23-A27, U11-A22-A26. The ${ }^{1}$ H-NMR and temperature stability studies reported here show that the MGA-MGA structure is sensitive to $\mathrm{pH}$ and the salt concentration and composition of the buffer solution. The buffer conditions differed among the MD simulation ( $\mathrm{pH} 7.0,150 \mathrm{mM} \mathrm{Mg} \mathrm{Mg}^{2+}$ or $300 \mathrm{mM} \mathrm{KCl}$ ), RNase I footprinting (pH 7.2-7.4, $100 \mathrm{mM} \mathrm{KCl,} 5 \mathrm{mM} \mathrm{MgCl}$ ), $\mathrm{NMR}$ (pH 5.8, $10 \mathrm{mM}$ $\mathrm{KCl})$ and crystallography ( $\mathrm{pH} 4.5,23 \mathrm{mM} \mathrm{KCl}, 40 \mathrm{mM} \mathrm{SrCl} 2,6.7 \mathrm{mM} \mathrm{MgCl}_{2}$ ). These results suggest that the discrepancies of stem I structure obtained by the different methods could be due to differences in the conditions of measurements.

There is only a U11-A22 base pair in the binding pocket of unoccupied MGA in the buffer condition for 3D- structure determination by $\mathrm{NMR}^{34}$ but the MD simulation showed base triples of C10-G23-A27, U11-A22-A26, G24-G29-A31 and base pair of G8-C28 in unoccupied MGA. Evidence of this pre-organization of the binding pocket in unoccupied MGA was found by RNase I footprinting. In the MGA-MG complex, the binding pocket was maintained through the simulation with some changes and a broader dynamic range compared to what was found by 3D-NMR. For example, in MD simulation the G24 was much more dynamic in the C7-G29-G24-A31 base quadruple (data not shown) compared to that in the 3D NMR structure.

Overall, we found that the MD simulation is more consistent with the RNase I footprinting results than the NMR and crystal structures. The most likely reason for these differences may be the buffer conditions used, and particularly the $\mathrm{pH}$. Both the MD simulation and the footprinting analysis were done at neutral $\mathrm{pH}$ whereas the NMR and crystal structures were performed with the aptamertarget complex at $\mathrm{pH} 5.8$ and 4.5. In addition, $\mathrm{Mg}^{2+}$ and $\mathrm{K}^{+}$also play roles. 
The MD simulation also gave new insight into the dynamics of the MG-MGA interaction. For example, the global conformational change of the MGA upon MG binding due to the twisting of the MGA along the binding pocket and stem II was not readily evident from RNase I footprinting of MGA and the 3D-structure of MGA-MG and MGA-TMR determined by NMR and crystallography. Similar phenomena of global structure change due to twisting of an aptamer on target binding has also been reported in NFKB-aptamer interaction ${ }^{3}$. An advantage of MD simulation is that it is able to generate simulated 3D structures of molecules such as the MGA that are very mobile. Whereas, for experimental applications such as NMR or crystallography it is necessary to identify buffer conditions that stabilize a more narrow range of molecular structures and thus limit molecular motion in order to obtain interpretable data.

\section{A model for the mechanism by which MGA binds MG}

Three models of aptamer-target interactions have been proposed, including adaptive recognition (induced fit) ${ }^{4}$, preorganized structure (lock and key) ${ }^{21}$ and the combination of preorganization-induced fit ${ }^{3 ; 10 ; 13 ; 14}$. According to these models, two kinetics pathways are possible for aptamer-target interactions. The pathway for a "lock and key" mechanism is a single step reaction with the target fitting precisely into the preorganized pocket. For the induced fit mechanism, there is an intermediate complex of the aptamer and target molecule in which the binding pocket is not organized for target binding. After the two molecules interact in this intermediate form the aptamer reorganizes to create a suitable high affinity binding pocket.

The thermodynamic and kinetic constants for the MGA-MG interaction suggest a structural rearrangement during the MGA-MG interaction. The ITC analysis showed that the MGA-MG interaction had a favorable enthalpy and unfavorable entropy. From this it could be proposed that MG binding to the MGA involves a structural rearrangement for which the entropy penalty is compensated for by a favorable enthalpy. This proposal is consistent with our footprinting data of 
MGA and the MGA-MG complex. An induced-fit mechanism was also proposed on the basis of the observed increased base-pairing in the binding pocket of MGA on MG binding ${ }^{34}$. However, neither footprinting nor NMR data can rule out the lock and key model.

Short RNAs can be very dynamic and heterogeneous in conformation and this feature can sometimes be observed after they are resolved by polyacrylamide gel electrophoresis (PAGE) in the absence of denaturants. MGA showed one major band and two minor bands when resolved through a $10 \%$ polyacrylamide gel thus suggesting some heterogeneity in structure (data not shown).

Therefore, it is possible that a minor population (e.g. 1\%) of the MGA exists in a form that has a completely preformed pocket and that cannot be detected by NMR or footprinting. Thus, a structural rearrangement in the MGA could involve equilibration amongst unoccupied MGA structures or a structural rearrangement in the MGA-MG complex after its initial formation. H-bond analysis of the simulated MGA structures showed no preexisting C7-G29 base pairing in the MGA. Thus, the simulation predicts that the rearrangement occurs post MG binding.

A proposed kinetic pathway for formation of the MG-MGA complex, shown in figure 8, invokes a mixed pre-organized and induced-fit model for the MG binding pocket. In the model, the MG interacts with a partially organized binding pocket on the MGA and induces the formation of the mature binding pocket by expelling the $\mathrm{C} 7$ sitting in the pocket. The binding pocket then reorganizes around the MG which becomes stacked between the G8-C28 base pair and the newly formed base quadruple of C7-G29-G24-A31. The process of optimizing the MG position in the reorganized binding pocket results in twisting of the MGA along its binding pocket and stem II to form the equilibrium MGA-MG complex.

\section{The value of MD simulation for modeling unavailable empty aptamer structures}

Due to the dynamic nature of short nucleic acids, the 3D-structures of aptamers are difficult to obtain experimentally either by NMR or crystallography. Although more than 40 experimentally 
determined 3D-structures of aptamer-target complexes are available, only a few 3D-structures of unoccupied aptamers have been reported to our knowledge. In this study, we developed a simulated 3D-structure of unoccupied MGA for which experimental 3D-structure is not available. The modeled structure was consistent with interpretations of experiment data from RNase I structure probing, melting profiles, thermodynamic and kinetic measurements. The strategy used here may be generally useful for understanding the 3D-structures of unoccupied aptamers and for studying the dynamics of aptamer-target interactions.

\section{MATERIALS AND METHODS}

\section{Materials}

The following describes the sources of reagents used in these studies: SalI and XbaI (New England Biolab), T4 polynucleotide kinase, RNase I and calf intestine alkaline phosphatase (Promega,

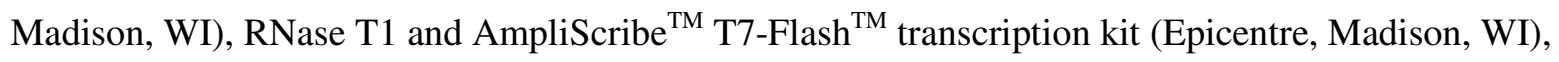
Taq DNA polymerase (GenScript, Piscataway, NJ), dNTP and NTP (Fermentas, Glen Burnie, MD), $\gamma-{ }^{32}$ P-ATP (MP Biomedicals, Irvine, CA), MG and tetramethylrosamine (TMR) (Sigma, St. Loius, MO), SYBR Green I (Invitrogen, Carlsband, CA) and other chemicals (Fisher, Suwanee, GA). The following oligonucleotides were chemically synthesized with standard desalting by either DNA Synthesis and Sequencing Facility (Iowa State University) or Integrated DNA Technology (Coralville, IA): MGA (38 nt RNA): 5'-ggaucccgacuggcgagagccagguaacgaauggaucc-3'; oligo 1: 5' -aaaaacggatccegactggcgagagccaggtaacgaatggatcct-3'; oligo 2: 5'-tttttaggatccattcgttacctggctctcgccagtcgggatccg3'; oligo 3: 5'-tcgacggatcccgactggcgagagccaggtaacgaatggatcct-3'; oligo 4: 5'-ctagaggatccatt cgttacctggctctcgccagtcgggatcgc-3'; primer 1: 5'-gtccgctctagaggatccattcg-3'; primer 2: 5'taatacgactcactatagggagcagcacgtcgacggatcccgac--3' 


\section{Molecular dynamics simulations}

MD simulation of the MGA- MG complex and the unoccupied MGA were carried out with NAMD2.6, which was developed by the Theoretical and Computational Biophysics Group in the Beckman Institute for Advanced Science and Technology at the University of Illinois at UrbanaChampaign (http://www.ks.uiuc.edu/Research/namd/) ${ }^{36}$. The setup for simulation and analysis of the simulation results was done with VMD1.8.6 (http://www.ks.uiuc.edu/Research/vmd/) ${ }^{37}$. The CHARMM topology file (top_all27_prot_na.rtf) and the parameter file (par_all27_prot_na.prm) ${ }^{38}$ were modified to include MG for setting up and running the simulation. For MG, the topology and parameter files were generated manually according to previously reported quantum-chemical calculations ${ }^{39}$.

The procedure is described briefly as follows. The MGA-MG complex (PDB ID: 1Q8N, model 1) ${ }^{34}$ and the MGA was derived from the MGA-MG complex by manually deleting MG were

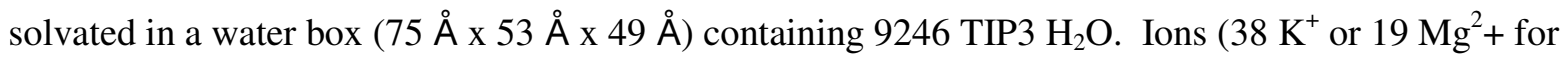
MGA and $38 \mathrm{~K}^{+}, 1 \mathrm{Cl}^{-}$or $19 \mathrm{Mg}^{2+}, 1 \mathrm{Cl}^{-}$for MGA-MG) were randomly added into the solvated system using the autoionization plugin in VMD. The solvated and ionized systems were then run on NAMD with CHARMM. For the simulation parameters, the electrostatic and van der Waals interaction distance cutoff was set to $12 \AA$. The full-system periodic electrostatics was calculated with a particle mesh Ewald summation of $\sim 1 \AA$ Arid space. The timestep was set to 2 fs. After 1002000 steps of minimization, the unconstrained simulation was run under a periodic boundary condition at a constant temperature (298K) and pressure (1.01325 bar) for 3 ns for MGA-MG solvated with $\mathrm{K}^{+}, 7 \mathrm{~ns}$ for MGA-MG solvated with $\mathrm{Mg}^{2+}$ and $7.7 \mathrm{~ns}$ for MGA solvated with $\mathrm{Mg}^{2+}$.

\section{Plasmid construction and in vitro transcription}

To generate the RNA molecule, 4MGA, that contains four tandem units of MGA, the plasmid, p4MG, was constructed by inserting four co-annealed oligonucleotides, oligo 1-4, into the plasmid 
ptz'U6 cut with SalI and XbaI. When co-annealed, these four oligonucleotides encoded an RNA with four tandem MGA each separated by a $7 \mathrm{nt}$ linker. The obtained plasmid containing the 4MGA sequence inserted downstream from a T7 polymerase promoter was called p4MG.

To obtain the RNA, the 4MGA DNA in the p4MG plasmid was amplified by polymerase chain reaction (PCR). In the PCR reaction mix was $4 \mathrm{ng} / \mu \mathrm{l}$ p4MG, $0.5 \mu \mathrm{M}$ primer $1,0.5 \mu \mathrm{M}$ primer 2 , $200 \mu \mathrm{M}$ dNTPs, $0.025 \mathrm{U} / \mu \mathrm{l}$ Taq DNA polymerase, $1.5 \mathrm{mM} \mathrm{MgCl}_{2}, 50 \mathrm{mM} \mathrm{KCl}, 10 \mathrm{mM}$ Tris (pH 9.0) and $0.1 \%$ Triton $\mathrm{X}-100$ in $500 \mathrm{ul}$ reaction. The reaction was amplified at $94^{\circ} \mathrm{C}, 3 \mathrm{~min} ; 20$ cycles of $94^{\circ} \mathrm{C} 30 \mathrm{sec}, 68^{\circ} \mathrm{C} 30 \mathrm{sec}, 72^{\circ} \mathrm{C} 1 \mathrm{~min} ; 72^{\circ} \mathrm{C}, 7 \mathrm{~min}$. The 4 MGA-DNA was then used for in vitro transcription by T7 RNA polymerase to generate 4MGA RNA. The 4MGA RNA was 202 nt long with sequence of 5'-gggagcagcacgucgacggaucccgacuggcgagagccagguaacgaauggauccuaaaaacggaucccgacuggcgagagecagguaacgaauggauccuaaaaacggaucccgacuggcgagagccagguaacgaatggauccuaaaaacggaucccgacuggcgagagccaggtaacgaauggauccucuagagcggac -3 '.

\section{RNase I footprinting}

Structural changes upon MG binding to MGA and 4MGA were studied by RNase I footprinting. To label the 4MGA RNA, it was first dephosphorylated. A $50 \mu$ l reaction mixture containing $20 \mu \mathrm{M}$ 4MGA, $0.2 \mathrm{U} / \mu \mathrm{l}$ calf intestine alkaline phosphatase, $50 \mathrm{mM}$ Tris $\mathrm{pH} 9.3,1 \mathrm{mM}$ $\mathrm{MgCl}_{2}, 0.1 \mathrm{mM} \mathrm{ZnCl}_{2}, 1 \mathrm{mM}$ spermidine was incubated at $37^{\circ} \mathrm{C}$ for $30 \mathrm{~min}$. Chemically synthesized MGA had no 5' phosphate. MGA and 4MGA were radiolabeled by 5' end labeling, which was carried out in $20 \mathrm{ul}$ reaction mixtures of $7 \mu \mathrm{M}$ MGA or 4MGA, $1 \mathrm{U} / \mu \mathrm{l} \mathrm{T} 4$ polynucleotide kinase, 2 $\mu \mathrm{Ci} / \mu \mathrm{l} \gamma-{ }^{32} \mathrm{P}$-ATP, $70 \mathrm{mM}$ Tris, $\mathrm{pH} 7.6,10 \mathrm{mM} \mathrm{MgCl} 2$ and $5 \mathrm{mM}$ DTT at $37^{\circ} \mathrm{C}$ for $1.5 \mathrm{~h}$. Following 5' end-labeling, RNase I footprinting was carried out. In the titration footprinting, 30-100 nM

${ }^{32} \mathrm{P}-\mathrm{MGA}$ or ${ }^{32} \mathrm{P}-4 \mathrm{MGA}$ in $100 \mathrm{mM} \mathrm{KCl}, 5 \mathrm{mM} \mathrm{MgCl} 2,10 \mathrm{mM}$ HEPES, $\mathrm{pH} 7.2,25{ }^{\circ} \mathrm{C}$ were treated with $10^{-4} \mathrm{U} / \mu \mathrm{l}$ and $5 \times 10^{-4} \mathrm{U} / \mu 1 \mathrm{RNase}$ I respectively in the presence of a range of malachite green concentrations $(0.1$ to $4 \mu \mathrm{M})$ each in $10 \mu \mathrm{l}$ reaction mixtures. For the controls, partial alkaline 
hydrolysis of the labeled RNAs was done in $50 \mu \mathrm{M} \mathrm{Na}_{2} \mathrm{CO}_{3}, \mathrm{pH} 9.0$ at $95{ }^{\circ} \mathrm{C}$ for 2 to $5 \mathrm{~min}$. RNase T1 digestion of the labeled RNAs was carried out in $10 \mu 1$ solution containing $0.5-1 \mathrm{U} / \mu \mathrm{l}$ Rase T1, 5 $\mathrm{M}$ urea, $350 \mathrm{mM}$ sodium citrate, $0.7 \mathrm{mM}$ EDTA, $\mathrm{pH} 5.0$, at $50^{\circ} \mathrm{C}$ for $4 \mathrm{~min}$. The RNA hydrolysis/cleavage reactions were stopped by bringing the reaction mixtures to $47.5 \%$ formamide, $0.05 \%$ bromophenol blue, and $0.05 \%$ xylene cyanol FF. The samples were analyzed by $7 \mathrm{M}$ urea 8 $12 \%$ polyacrylamide gels. The gels were dried and the radioactivity was recorded using a phosphor screen that was imaged with a Typhoon 8600 Variable Model Imager (GE Healthcare, Piscataway, NJ). The image data were quantified by ImageQuant (GE Healthcare) and fit to equation $Y=$ $\mathrm{a} *[\mathrm{M}] /\left(\mathrm{K}_{\mathrm{D}}+[\mathrm{M}]\right)+\mathrm{b}$ by nonlinear regression with Costat (Cohort, $\left.\mathrm{CA}\right)$. In the equation, Y presented the normalized cleavage against that in the absence of MG. [M] was MG concentration. $K_{D}$ was dissociation constant of MG-MGA interaction. The a and b were constants. The lanes of partial alkaline hydrolysis and RNase T1 digestion were used for identification of cleavage bands.

\section{${ }^{1}$ H-NMR spectrometry}

To test for effects of buffer conditions ( $\mathrm{pH}$ and salts) on the structures of MGA and the MGA-MG complex, one-dimensional NMR spectra of MGA-(MG) were recorded at $25{ }^{\circ} \mathrm{C}$ for $50 \mu \mathrm{M}$ $\mathrm{MGA} \pm 100 \mu \mathrm{M}$ MG in $5 \% \mathrm{D}_{2} \mathrm{O}$ and $95 \% \mathrm{H}_{2} \mathrm{O}$ including $10 \mathrm{mM} \mathrm{KCl}, 10 \mathrm{mM} \mathrm{KH}_{2} \mathrm{PO}_{4}, \mathrm{pH} 5.8 ; 10$ $\mathrm{mM} \mathrm{KCl}, 10 \mathrm{mM} \mathrm{KH}_{2} \mathrm{PO}_{4}, \mathrm{pH} 7.2 ; 10 \mathrm{mM} \mathrm{KCl}, 10 \mathrm{mM} \mathrm{KH}_{2} \mathrm{PO}_{4}, 5 \mathrm{mM} \mathrm{MgCl}_{2}, \mathrm{pH} 5.8 ; 100 \mathrm{mM} \mathrm{KCl}$, $10 \mathrm{mM} \mathrm{KH}_{2} \mathrm{PO}_{4}, \mathrm{pH}$ 5.8. Spectra were collected using a Bruker Avance 700 spectrometer equipped with a $5 \mathrm{~mm}$ HCN-Z gradient cryoprobe. All 1D ${ }^{1} \mathrm{H}-\mathrm{NMR}$ spectra were acquired using a WATERGATE pulse sequence with water flipback (Bruker sequence p3919fpgp) to minimize solvent saturation transfer. Spectra were averages of 512 transients. Spectral width was $18182 \mathrm{~Hz}$ (25 ppm).

\section{Melting profiles}

The melting profiles of the MGA with and without MG were determined under different buffer conditions using a MiniOpticon real-time PCR system (BIO-RAD, Hercules, CA) in the 
temperature range of $30-90^{\circ} \mathrm{C}$ with a $0.5^{\circ} \mathrm{C}$ increase per step. The samples contained $2 \mu \mathrm{M}$ MGA and $0.7 x$ SYBR Green I with or without $20 \mu \mathrm{M}$ MG, in solutions containing $10 \mathrm{mM} \mathrm{KCl}, 10 \mathrm{mM}$ $\mathrm{KH}_{2} \mathrm{PO}_{4}, \mathrm{pH} 5.8 ; 10 \mathrm{mM} \mathrm{KCl}, 10 \mathrm{mM} \mathrm{KH}{ }_{2} \mathrm{PO}_{4}, \mathrm{pH}$ 7.2; $10 \mathrm{mM} \mathrm{KCl}, 10 \mathrm{mM} \mathrm{KH} \mathrm{PO}_{4}, 5 \mathrm{mM} \mathrm{MgCl}$, pH 5.8; $100 \mathrm{mM} \mathrm{KCl}, 10 \mathrm{mM} \mathrm{KH} \mathrm{PO}_{4}$, pH 5.8 or $100 \mathrm{mM} \mathrm{KCl,} 10 \mathrm{mM}$ HEPES, $5 \mathrm{mM} \mathrm{MgCl}$, pH 7.2. The measure of base stacking was the fluorescence of SYBR Green due to its being stacked between bases.

\section{Isothermal titration calorimetry (ITC)}

The thermodynamics of the MG-MGA interaction was determined by ITC with VP-ITC (MicroCal, Northampton, MA). For ITC, $300 \mu \mathrm{l}$ of $75 \mu \mathrm{M} \mathrm{MG}$ or TMR in $100 \mathrm{mM} \mathrm{KCl,} 5 \mathrm{mM}$ $\mathrm{MgCl}_{2}, 10 \mathrm{mM}$ HEPES, $\mathrm{pH} 7.0$ or $250 \mu \mathrm{M} \mathrm{MG}$ in $\mathrm{H}_{2} \mathrm{O}$ were in the syringe and titrated into $1.428 \mathrm{ml}$ of $10 \mu \mathrm{M}$ MGA in same buffer in sample cell at $25^{\circ} \mathrm{C}$. The titrations of $75 \mu \mathrm{M}$ MG or TMR, or 250 $\mu \mathrm{M}$ MG into same buffer served as the blank. A total of 30 injections were performed with an initial $1 \mu \mathrm{l}$ injection followed by $10 \mu \mathrm{l}$ injections and separated by a $60 \mathrm{sec}$ initial delay. Each $10 \mathrm{ul}$ injection was of $20 \mathrm{sec}$ duration and $300 \mathrm{sec}$ spacing. Other parameter settings included a $2 \mathrm{sec}$ filter period, $15 \mu \mathrm{cal} / \mathrm{sec}$ reference power, $310 \mathrm{rpm}$ stir speed, high feedback model, fast equilibrium \& auto ITC equilibration. The ITC raw data were analyzed using Origin7.0 software provided by MicroCal Inc. with the "one set of sites" fitting model.

\section{Stopped- flow kinetics}

The MGA-MG interaction was studied by stopped-flow kinetics using a MOS-250 spectrometer linked to a stopped-flow SFM-400 apparatus by a MPS-60 microprocessor unit (BioLogic, Claix, France). The MGA-MG interaction was monitored by quantifying the shift in maximal absorption of MG from $618 \mathrm{~nm}$ to $630 \mathrm{~nm}^{40}$. For $\mathrm{k}_{\mathrm{on}}$ measurements, MGA was mixed with MG in $100 \mathrm{mM} \mathrm{KCl}, 5 \mathrm{mM} \mathrm{MgCl} 2,10 \mathrm{mM}$ HEPES, $\mathrm{pH} 7.2$ at $25^{\circ} \mathrm{C}$ with final concentrations of MGA as 20, $25,30,35$ or $40 \mu \mathrm{M}$ and $\mathrm{MG}$ as $2 \mu \mathrm{M}$. The binding was monitored by the change in the absorbance at 
$613 \mathrm{~nm}$ (A613) over the period from 20 to $120 \mathrm{msec}$ after mixing. For $\mathrm{k}_{\mathrm{off}}$ experiments, a mixture of MGA and MG was mixed at $\mathrm{t}=0$ with TMR to a final concentration of $2 \mu \mathrm{M} \mathrm{MG}, 3 \mu \mathrm{M}$ MGA, $50 \mu \mathrm{M}$ TMR. The MGA has a much higher affinity for TMR than for MG (40-90 nM vs. 0.2-0.8 $\mu \mathrm{M})$.

Therefore, TMR in excess was used to prevent the reassociation of MGA and MG. The dissociation of MG from MGA was tracked by the change of absorbance at $632 \mathrm{~nm}$ (A632) in the periods of 20 msec to $20 \mathrm{sec}$, which is not affected by TMR absorbance $(\lambda \max =551 \mathrm{~nm}$ for TMR and $567 \mathrm{~nm}$ for TMR-MGA). The kinetic data were fit with an exponential function using Pro5.03 software to obtain $\mathrm{k}_{\mathrm{off}}$ and $\mathrm{k}_{\mathrm{obs}} . \mathrm{k}_{\mathrm{on}}$ is the slope of the linear curve $\mathrm{k}_{\mathrm{obs}}=\mathrm{k}_{\mathrm{on}} *[\mathrm{MGA}]+\mathrm{c}$ where $[\mathrm{MGA}]$ is the concentrations of MGA and $\mathrm{c}$ is a constant.

\section{ACKNOWLEDGEMENT}

This work was supported by grants from the NIH (R01HL078659) and the Office of Energy Science Research, Office Medical Sciences, Life Sciences of the U. S. Department of Energy under Contracts W-7405-Eng-82 with the Ames National Laboratories.

\section{REFERENCES}

1. Marathias, V. M., Wang, K. Y., Kumar, S., Pham, T. Q., Swaminathan, S. \& Bolton, P. H. (1996). Determination of the number and location of the manganese binding sites of DNA quadruplexes in solution by EPR and NMR in the presence and absence of thrombin. $J$ Mol Biol 260, 378-94.

2. Bouvet, P., Allain, F. H., Finger, L. D., Dieckmann, T. \& Feigon, J. (2001). Recognition of pre-formed and flexible elements of an RNA stem-loop by nucleolin. J Mol Biol 309, 763-75.

3. Reiter, N. J., Maher, L. J., 3rd \& Butcher, S. E. (2008). DNA mimicry by a high-affinity antiNF-kappaB RNA aptamer. Nucleic Acids Res 36, 1227-36.

4. Hermann, T. \& Patel, D. J. (2000). Adaptive recognition by nucleic acid aptamers. Science $287,820-5$. 
5. Wakeman, C. A., Winkler, W. C. \& Dann, C. E., 3rd. (2007). Structural features of metabolite-sensing riboswitches. Trends Biochem Sci 32, 415-24.

6. Berman, H. M., Westbrook, J., Feng, Z., Gilliland, G., Bhat, T. N., Weissig, H., Shindyalov, I. N. \& Bourne, P. E. (2000). The Protein Data Bank. Nucleic Acids Res 28, 235-42.

7. Greenleaf, W. J., Frieda, K. L., Foster, D. A., Woodside, M. T. \& Block, S. M. (2008). Direct observation of hierarchical folding in single riboswitch aptamers. Science 319, 630-3.

8. Yu, J., Jiang, Y., Ma, X., Lin, Y. \& Fang, X. (2007). Energy landscape of aptamer/protein complexes studied by single-molecule force spectroscopy. Chem Asian J 2, 284-9.

9. $\quad$ Nick Taylor, J., Darugar, Q., Kourentzi, K., Willson, R. C. \& Landes, C. F. (2008). Dynamics of an anti-VEGF DNA aptamer: a single-molecule study. Biochem Biophys Res Commun 373, 213-8.

10. Lemay, J. F., Penedo, J. C., Tremblay, R., Lilley, D. M. \& Lafontaine, D. A. (2006). Folding of the adenine riboswitch. Chem Biol 13, 857-68.

11. Wilkinson, T. A., Zhu, L., Hu, W. \& Chen, Y. (2004). Retention of conformational flexibility in HIV-1 Rev-RNA complexes. Biochemistry 43, 16153-60.

12. Nonin, S., Jiang, F. \& Patel, D. J. (1997). Imino proton exchange and base-pair kinetics in the AMP-RNA aptamer complex. J Mol Biol 268, 359-74.

13. Ottink, O. M., Rampersad, S. M., Tessari, M., Zaman, G. J., Heus, H. A. \& Wijmenga, S. S. (2007). Ligand-induced folding of the guanine-sensing riboswitch is controlled by a combined predetermined induced fit mechanism. Rna 13, 2202-12.

14. Noeske, J., Buck, J., Furtig, B., Nasiri, H. R., Schwalbe, H. \& Wohnert, J. (2007). Interplay of 'induced fit' and preorganization in the ligand induced folding of the aptamer domain of the guanine binding riboswitch. Nucleic Acids Res 35, 572-83. 
15. Buck, J., Furtig, B., Noeske, J., Wohnert, J. \& Schwalbe, H. (2007). Time-resolved NMR methods resolving ligand-induced RNA folding at atomic resolution. Proc Natl Acad Sci U S A 104, 15699-704.

16. Robertson, S. A., Harada, K., Frankel, A. D. \& Wemmer, D. E. (2000). Structure determination and binding kinetics of a DNA aptamer-argininamide complex. Biochemistry $39,946-54$

17. Gilbert, S. D., Stoddard, C. D., Wise, S. J. \& Batey, R. T. (2006). Thermodynamic and kinetic characterization of ligand binding to the purine riboswitch aptamer domain. $J \mathrm{Mol}$ Biol 359, 754-68.

18. Lang, K., Rieder, R. \& Micura, R. (2007). Ligand-induced folding of the thiM TPP riboswitch investigated by a structure-based fluorescence spectroscopic approach. Nucleic Acids Res 35, 5370-8.

19. Jucker, F. M., Phillips, R. M., McCallum, S. A. \& Pardi, A. (2003). Role of a heterogeneous free state in the formation of a specific RNA-theophylline complex. Biochemistry 42, 2560-7.

20. Muller, M., Weigand, J. E., Weichenrieder, O. \& Suess, B. (2006). Thermodynamic characterization of an engineered tetracycline-binding riboswitch. Nucleic Acids Res 34, $2607-17$

21. Tinsley, R. A., Furchak, J. R. \& Walter, N. G. (2007). Trans-acting glmS catalytic riboswitch: locked and loaded. Rna 13, 468-77.

22. Bishop, G. R., Ren, J., Polander, B. C., Jeanfreau, B. D., Trent, J. O. \& Chaires, J. B. (2007). Energetic basis of molecular recognition in a DNA aptamer. Biophys Chem 126, 165-75.

23. Yamasaki, S., Nakamura, S., Terada, T. \& Shimizu, K. (2007). Mechanism of the difference in the binding affinity of E. coli tRNAGln to glutaminyl-tRNA synthetase caused by noninterface nucleotides in variable loop. Biophys J 92, 192-200. 
24. Hianik, T., Ostatna, V., Sonlajtnerova, M. \& Grman, I. (2007). Influence of ionic strength, $\mathrm{pH}$ and aptamer configuration for binding affinity to thrombin. Bioelectrochemistry 70, 12733.

25. Mori, T., Oguro, A., Ohtsu, T. \& Nakamura, Y. (2004). RNA aptamers selected against the receptor activator of NF-kappaB acquire general affinity to proteins of the tumor necrosis factor receptor family. Nucleic Acids Res 32, 6120-8.

26. Anderson, P. C. \& Mecozzi, S. (2005). Identification of a 14mer RNA that recognizes and binds flavin mononucleotide with high affinity. Nucleic Acids Res 33, 6992-9.

27. Schneider, C. \& Suhnel, J. (1999). A molecular dynamics simulation of the flavin mononucleotide-RNA aptamer complex. Biopolymers 50, 287-302.

28. Anderson, P. C. \& Mecozzi, S. (2005). Unusually short RNA sequences: design of a 13-mer RNA that selectively binds and recognizes theophylline. J Am Chem Soc 127, 5290-1.

29. Anderson, P. C. \& Mecozzi, S. (2006). Minimum sequence requirements for selective RNAligand binding: a molecular mechanics algorithm using molecular dynamics and free-energy techniques. J Comput Chem 27, 1631-40.

30. Gouda, H., Kuntz, I. D., Case, D. A. \& Kollman, P. A. (2003). Free energy calculations for theophylline binding to an RNA aptamer: Comparison of MM-PBSA and thermodynamic integration methods. Biopolymers 68, 16-34.

31. Anderson, P. C. \& Mecozzi, S. (2007). Minimum sequence requirements for the binding of paromomycin to the rRNA decoding site A. Biopolymers 86, 95-111.

32. Pagano, B., Martino, L., Randazzo, A. \& Giancola, C. (2008). Stability and binding properties of a modified thrombin binding aptamer. Biophys $J$ 94, 562-9.

33. Beaurain, F., Di Primo, C., Toulme, J. J. \& Laguerre, M. (2003). Molecular dynamics reveals the stabilizing role of loop closing residues in kissing interactions: comparison between TARTAR* and TAR-aptamer. Nucleic Acids Res 31, 4275-84. 
34. Flinders, J., DeFina, S. C., Brackett, D. M., Baugh, C., Wilson, C. \& Dieckmann, T. (2004). Recognition of planar and nonplanar ligands in the malachite green-RNA aptamer complex. Chembiochem 5, 62-72.

35. Baugh, C., Grate, D. \& Wilson, C. (2000). 2.8 A crystal structure of the malachite green aptamer. J Mol Biol 301, 117-28.

36. Phillips, J. C., Braun, R., Wang, W., Gumbart, J., Tajkhorshid, E., Villa, E., Chipot, C., Skeel, R. D., Kale, L. \& Schulten, K. (2005). Scalable molecular dynamics with NAMD. J Comput Chem 26, 1781-802.

37. Humphrey, W., Dalke, A. \& Schulten, K. (1996). VMD: visual molecular dynamics. J Mol Graph 14, 33-8, 27-8.

38. MacKerell, A. D., Jr., Banavali, N. \& Foloppe, N. (2000). Development and current status of the CHARMM force field for nucleic acids. Biopolymers 56, 257-65.

39. Nguyen, D. H., DeFina, S. C., Fink, W. H. \& Dieckmann, T. (2002). Binding to an RNA aptamer changes the charge distribution and conformation of malachite green. J Am Chem Soc 124, 15081-4.

40. Grate, D. \& Wilson, C. (1999). Laser-mediated, site-specific inactivation of RNA transcripts. Proc Natl Acad Sci U S A 96, 6131-6. 


\section{FIGURE LEGENDS}

\section{Figure 1. Dynamics of the MGA-MG interaction predicted by MD simulation.}

The distances between two atoms were calculated from the MD simulation as a function of simulated time. (a) H-bond (stem I and binding pocket) and base-stacking (tetraloop) distances. The atom pairs are stated at the top of each graph and the graphs are organized in the figure by location in the MGA structure. Distances are shown for these pairs in MGA (blue) and MGA-MG complex (red). (b) The alignment of the MGA (transparent) and the MGA-MG complex (opaque) along the stem II and binding pocket with the binding pocket silver, stem II blue, stem I green, tetraloop pink and MG (magenta).

Figure 2. The structural change of the MGA upon MG binding determined by RNase I footprinting.

RNase I footprinting is of the MGA in the presence and absence of 0.1 to $4 \mu \mathrm{M}$ MG shows changes in accessibility of RNase I to phosphodiester bonds in the binding pocket [X:A22-U32; ०:C7-U11], stem I ( $\square)$ and tetraloop $(\Delta)$. Four experiments were performed with two independently synthesized MGAs. A representative gel is shown in (a) and the quantitation of the four experiments as average is shown in (b). Y represents the cleavage by RNase I normalized against that at $0 \mu \mathrm{M} \mathrm{MG.}$

Figure 3. ${ }^{1}$ H-NMR spectra of the MGA, MGA-MG complex and MG under different buffer conditions.

Buffer compositions were based on the buffer $\mathrm{N}$ used for NMR analysis and all contained $10 \mathrm{mM}$ $\mathrm{KH}_{2} \mathrm{PO} 4$ with the variable constituents outlined in the table

Figure 4. The melting profiles of the MGA and the MG-MGA complex in different buffers. MGA was incubated in the presence or absence of MG in buffer $\mathrm{N}(10 \mathrm{mM} \mathrm{KCl}, 10 \mathrm{mM} \mathrm{KH} 2 \mathrm{PO}$, $\mathrm{pH}$ 5.8) that was used for the NMR structure determination or buffer $\mathrm{F}(100 \mathrm{mM} \mathrm{KCl}, 10 \mathrm{mM}$ HEPES, $5 \mathrm{mM} \mathrm{MgCl} 2, \mathrm{pH} \mathrm{7.2)} \mathrm{that} \mathrm{was} \mathrm{used} \mathrm{here} \mathrm{for} \mathrm{footprinting.} \mathrm{A} \mathrm{melting} \mathrm{profile} \mathrm{was} \mathrm{performed} \mathrm{using}$ SYBR green I to report the extent of base pairing as a function of temperature. In the figure, $\mathrm{N}$ and $\mathrm{F}$ 
in the parenthesis following MGA or MGA-MG shows the buffer. The averages of four independent experiments with four repeats in each independent experiment are presented. Note that, unlike A260 which increases with melting due to the loss of the hyperchromatic effect of base stacking, the fluorescence of SYBR green decreases with melting due to the loss of base pairs between which it can stack.

Figure 5. Twisting of the MGA is suggested by the RNase I footprinting of tandemly linked MGAs.

RNase I footprinting of 4MGA in the presence and absence of 0.1 to $20 \mu \mathrm{M}$ MG was performed five times with two independently synthesized preparations of 4MGA. A representative gel is shown.

\section{Figure 6. Thermodynamics of the MGA-MG interaction.}

The thermodynamic parameters for the MGA and MG interaction in a buffer containing $10 \mathrm{mM}$ HEPES, $100 \mathrm{mM} \mathrm{KCl}, 5 \mathrm{mM} \mathrm{MgCl} 2$, pH 7.0 were obtained by ITC. Top panel: The raw ITC data with the blank subtracted showing the heat released rate with time. Bottom panel: The integrated data from the top panel showing the normalized heat release plotted against the molar ratio of MG/MGA. Presented are the results of one of the three independent titrations.

\section{Figure 7. The kinetics of the MG-MGA interaction.}

The on and off rates of MGA-MG binding were determined by stopped-flow analysis as described in Methods with $2 \mu \mathrm{M}$ MG and varying concentrations of MGA $(20,25,30,35$ and $40 \mu \mathrm{M})$ for determining kon and $2 \mu \mathrm{M}$ MG, $3 \mu \mathrm{M}$ MGA and $50 \mu \mathrm{M}$ TMR for the koff determination. Left panel: The data used to derive kon. right panel: the data used to derive koff . Two independent experiments were performed to determine both kon and koff with the results of at least three time courses averaged for each individual curve.

\section{Figure 8. The model for the mechanism of MG-MGA recognition.}

The MGA and MGA*-MG structures are the averaged simulated 3D-structure at equilibrium. The MGA-MG intermediate is a hypothesized intermediate state that might occur during the transition 
from MGA to MGA*-MG. In this model MG first enters the partially preorganized binding space in MGA to form a MGA-MG intermediate. Then MG expels $\mathrm{C} 7$ from the preorganized binding space of MGA to induce the formation of the base quadruple (C7-G29-G24-A31), optimizing the binding pocket for MG occupation and simultaneously twisting the MGA along the binding pocket and stem II to form the final MGA*-MG. The individual structures were prepared with VMD1.8.6. The top part of the figure showed the whole molecule and the bottom part showed the base-quadruple (C7G29-G24-A31) and the base pair (G8-C28) between which MG binds. The MGA consists of stem I (green, transparent), binding pocket (opaque), stem II (blue, transparent) and tetraloop (pink, transparent). In the MGA binding pocket, C7 is colored blue, G24, G29 and A31 are yellow, and G8 and C28 are cyan all of which presented as Licorice form. The rest of the residues were presented as newribbon format with A9, U25, A30 and U32 colored as silver, base triple of C10-G23-A27 as orange and base triple of U11-A22-A26 as magenta2. MG is presented as licorice format and colored with magenta. 


\section{TABLES}

Table 1. The Tm values of MGA and MGA-MG in various buffers

\begin{tabular}{|c|c|c|c|c|c|}
\hline \multirow[b]{3}{*}{ buffers } & \multicolumn{2}{|c|}{ MGA } & \multicolumn{3}{|c|}{ MGA-MG } \\
\hline & $\operatorname{Tm}\left({ }^{\circ} \mathrm{C}\right)$ & t-test between & $\operatorname{Tm}\left({ }^{\circ} \mathrm{C}\right)$ & t-test between & Low temperature \\
\hline & & $\mathrm{B}-\mathrm{F}$ and $\mathrm{N}$ & & $\mathrm{B}-\mathrm{F}$ and $\mathrm{N}$ & Transition $\left({ }^{\circ} \mathrm{C}\right)$ \\
\hline $\mathrm{N}$ & $53.2 \pm 2.4$ & & $65.0 \pm 1.5$ & & \\
\hline B & $46.7 \pm 0.8$ & $\mathrm{P}=2.6 \times 10^{-7}$ & $57.6 \pm 4.9$ & $\mathrm{P}=4.9 \times 10^{-7}$ & \\
\hline $\mathrm{C}$ & $54.1 \pm 2.0$ & $P=0.22$ & $68.0 \pm 0.6$ & $\mathrm{P}=2.3 \times 10^{-5}$ & \\
\hline D & $51.1 \pm 2.9$ & $P=0.04$ & $60.6 \pm 1.0$ & $\mathrm{P}=1.7 \times 10^{-7}$ & \\
\hline $\mathrm{F}$ & $49.6 \pm 2.0$ & $\mathrm{P}=8.1 \times 10^{-6}$ & $65.9 \pm 1.8$ & $\mathrm{P}=0.04$ & $41.1 \pm 1.9$ \\
\hline
\end{tabular}

Transition temperatures were determined for the MGA in the presence and absence of MG under the following buffer conditions: N) $\left.10 \mathrm{mM} \mathrm{KCl,} 10 \mathrm{mM} \mathrm{KH}_{2} \mathrm{PO}_{4}, \mathrm{pH} 5.8, \mathrm{~B}\right) 10 \mathrm{mM} \mathrm{KCl}, 10 \mathrm{mM}$ $\left.\mathrm{KH}_{2} \mathrm{PO}_{4}, \mathrm{pH} 7.2, \mathrm{C}\right) 10 \mathrm{mM} \mathrm{KCl}, 10 \mathrm{mM} \mathrm{KH} \mathrm{PO}_{4}, 5 \mathrm{mM} \mathrm{MgCl}$, pH 5.8, D) $100 \mathrm{mM} \mathrm{KCl}, 10 \mathrm{mM}$ $\mathrm{KH}_{2} \mathrm{PO}_{4}$, pH 5.8 and F) $100 \mathrm{mM} \mathrm{KCl}, 10 \mathrm{mM}$ HEPES, $5 \mathrm{mM} \mathrm{MgCl}$, $\mathrm{pH}$ 7.2. Shown for the derived Tm and low temperature transition are the average temperatures \pm the standard deviation. Four independent experiments were performed under these conditions to obtain the results shown. 
Table 2. Thermodynamics of MGA-MG/TMR interaction determined by ITC

\begin{tabular}{|c|c|c|c|c|c|c|}
\hline & $\mathrm{N}$ & $\begin{array}{l}\mathrm{K}_{\mathrm{D}} \\
(\mu \mathrm{M})\end{array}$ & $\begin{array}{l}\Delta \mathrm{H} \\
(\mathrm{kcal} / \mathrm{mol})\end{array}$ & $\begin{array}{l}\Delta \mathrm{S} \\
(\mathrm{cal} / \mathrm{mol} / \mathrm{deg})\end{array}$ & $\begin{array}{l}\Delta \mathrm{G} \\
(\mathrm{kcal} / \mathrm{mol})\end{array}$ & $\mathrm{R}^{2}$ \\
\hline MGA-MG & $0.65 \pm 0.04$ & $0.33 \pm 0.09$ & $-24.7 \pm 0.97$ & $-53.2 \pm 2.6$ & $-8.88 \pm 0.23$ & 0.995 \\
\hline MGA-MG * & $1.64 \pm 0.05$ & 2.14 & $-20.19 \pm 0.86$ & -41.8 & -7.73 & 0.990 \\
\hline MGA-TMR & $1.10 \pm 0.02$ & $0.095 \pm 0.005$ & $-15.3 \pm 0.60$ & $-19.1 \pm 2.5$ & $-8.95 \pm 0.13$ & 0.995 \\
\hline
\end{tabular}

Thermodynamic constants of MGA-target interaction were determined in buffer $(100 \mathrm{mM} \mathrm{KCl}, 5 \mathrm{mM}$ $\mathrm{MgCl} 2,10 \mathrm{mM}$ HEPES, $\mathrm{pH} 7.0$ ) or in $\mathrm{H} 2 \mathrm{O}(*)$. Each column in the table shows $\mathrm{N}$ : number of binding site, $\mathrm{K}_{\mathrm{D}}$ : dissociation constant, $\mathrm{H}$ : enthalpy, $\mathrm{S}$ : entropy, $\mathrm{G}$ : free energy and $\mathrm{R}^{2}$ : onesite model fitness 
Table 3. Comparing the MD simulations with the NMR and crystal structures

\begin{tabular}{|c|c|c|c|c|c|}
\hline & NMR & $\begin{array}{l}\text { MGA-MG- } \\
\mathrm{Mg}^{2+}\end{array}$ & $\begin{array}{l}\text { MGA-MG- } \\
\mathrm{K}^{+}\end{array}$ & $\begin{array}{l}\text { MGA- } \\
\mathrm{Mg}^{2+}\end{array}$ & $\begin{array}{l}\text { MGA-TMR } \\
\text { (crystal) }\end{array}$ \\
\hline NMR & & 2.745 & 2.604 & 2.524 & 2.698 \\
\hline MGA-MG-Mg ${ }^{2+}$ & 3.780 & & 1.889 & 1.902 & 2.869 \\
\hline MGA-MG-K ${ }^{+}$ & 3.463 & 2.302 & & 2.113 & 2.543 \\
\hline $\mathrm{MGA}^{-\mathrm{Mg}^{2+}}$ & 4.404 & 2.098 & 3.407 & & 2.903 \\
\hline MGA-TMR (crystal) & 4.070 & 3.876 & 3.805 & 4.546 & \\
\hline
\end{tabular}

The averaged NMR-derived MGA-MG structures, MGA-TMR crystal structure and averaged simulation structures were compared for identity with the RMSD being an indicator of the fit. A larger RMSD indicates a bigger difference between two structures. The RMSD values above the shaded areas are from comparisons of the binding pocket structure of the MGA alone and RMSD values below the shaded areas are from comparisons of the whole MGA structure. All atoms except the hydrogens were compared. 


\section{FIGURES}

Fig. 1

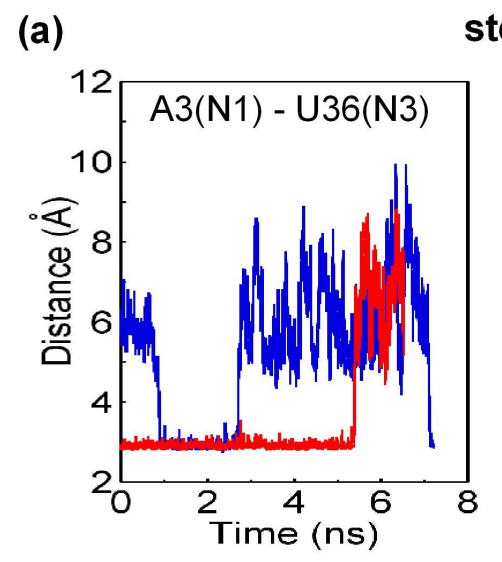

stem I

binding pocket
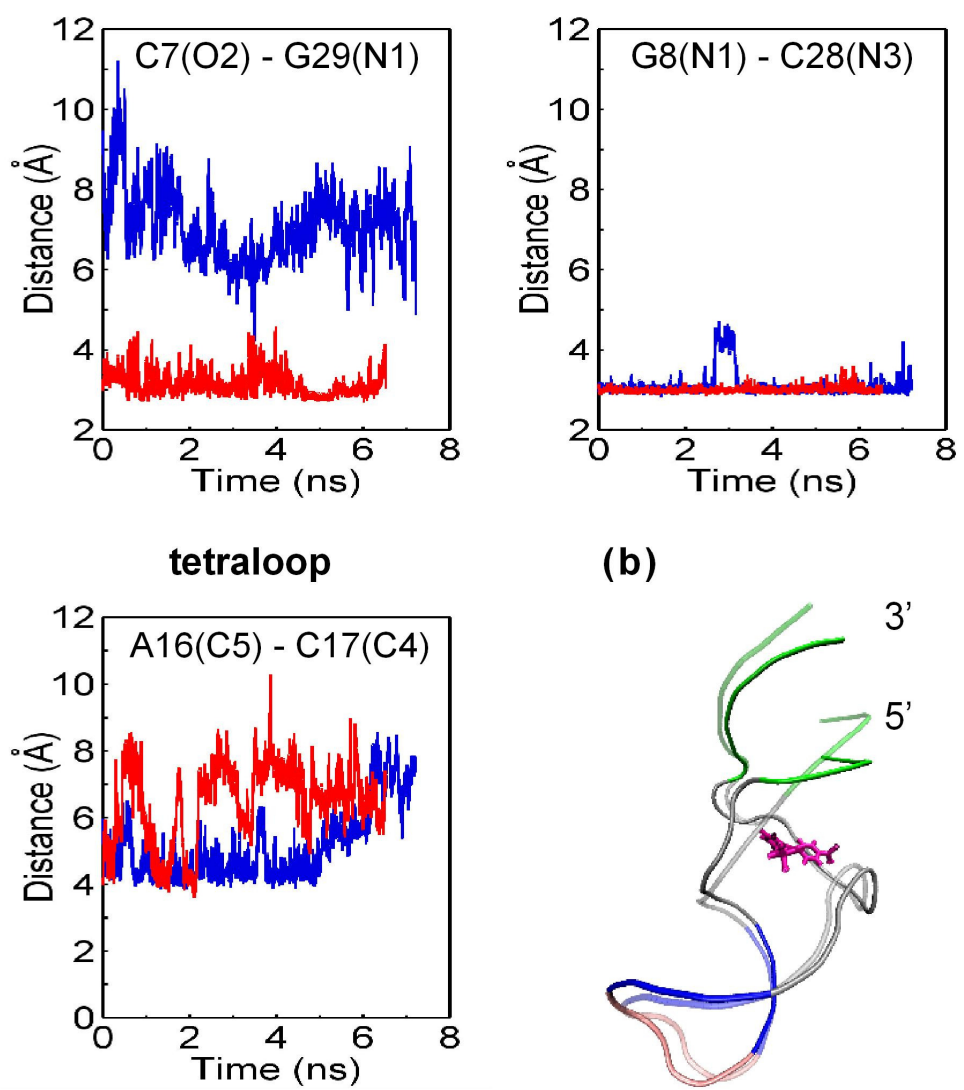

(b)

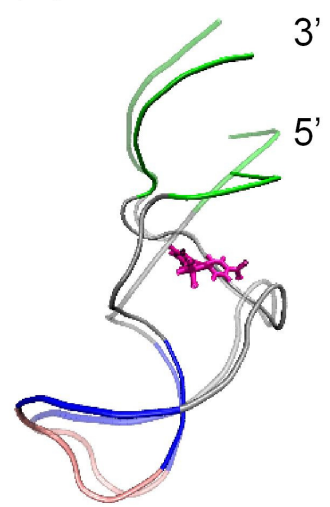


Fig. 2

(a)

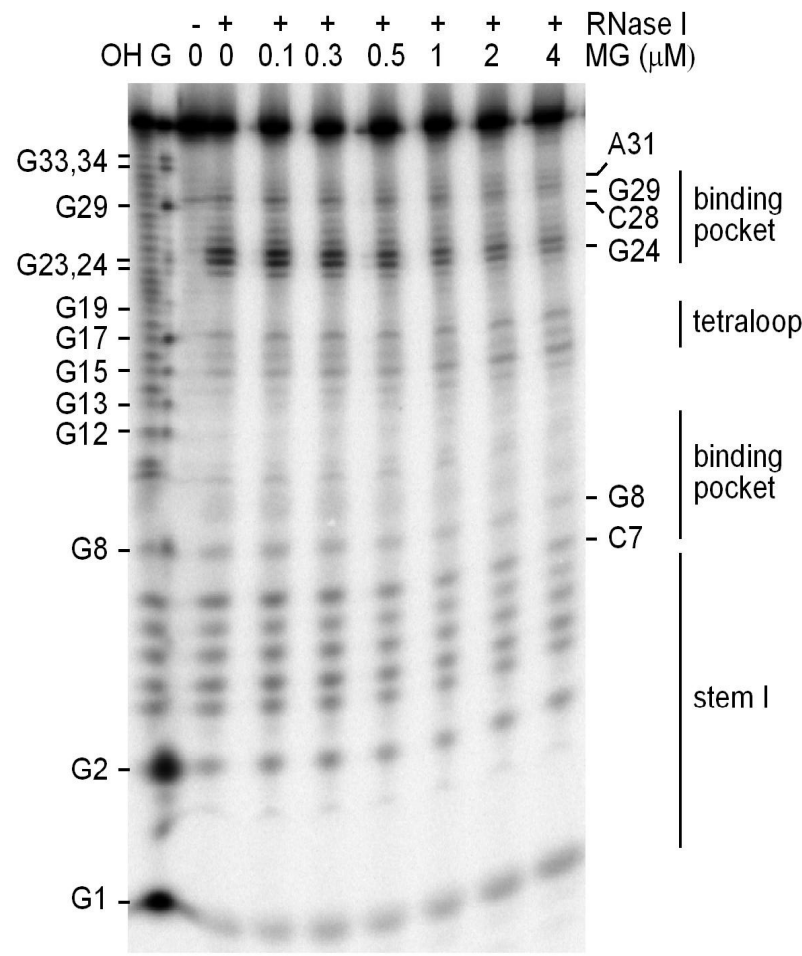

(b)

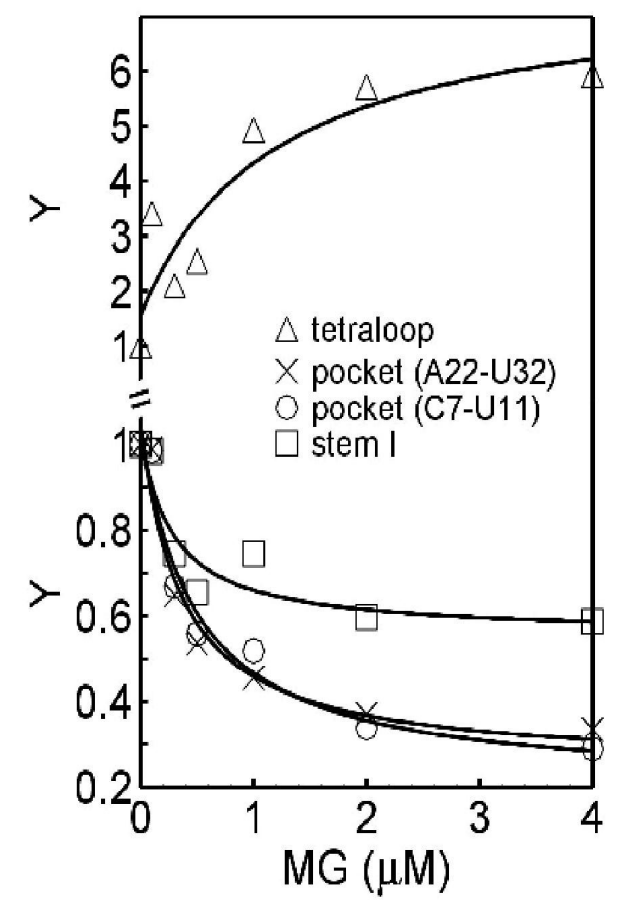


Fig. 3

\begin{tabular}{|c|c|c|c|}
\hline buffer & $\mathrm{pH}$ & $\mathrm{KCl}$ & $\mathrm{MgCl}_{2}$ \\
\hline $\mathrm{N}$ & 5.8 & $10 \mathrm{mM}$ & 0 \\
\hline $\mathrm{A}$ & 7.2 & $10 \mathrm{mM}$ & 0 \\
\hline $\mathrm{B}$ & 5.8 & $10 \mathrm{mM}$ & $5 \mathrm{mM}$ \\
\hline $\mathrm{C}$ & 5.8 & $100 \mathrm{mM}$ & 0 \\
\hline
\end{tabular}

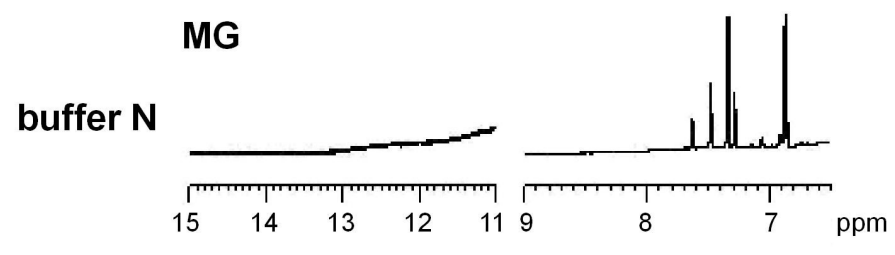

MGA

MGA-MG complex

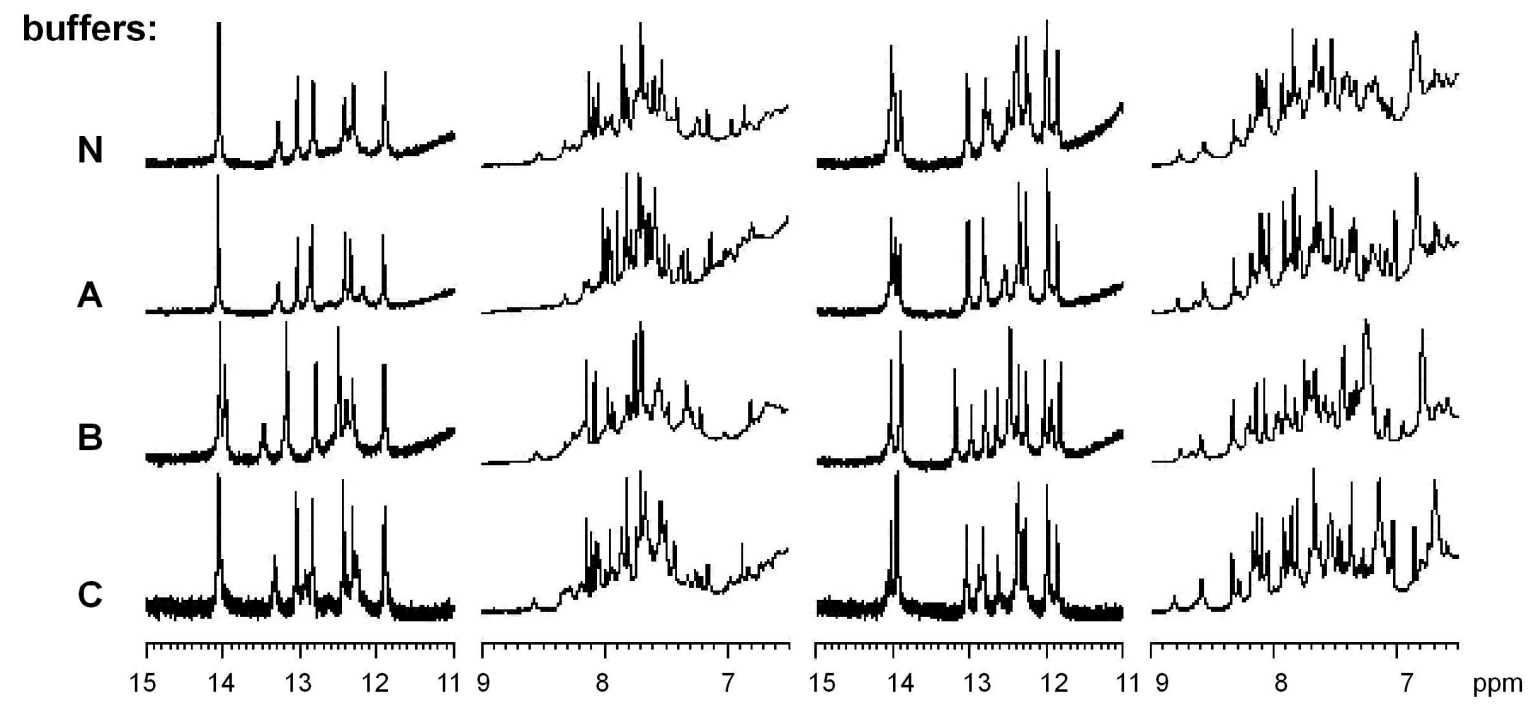


Fig. 4

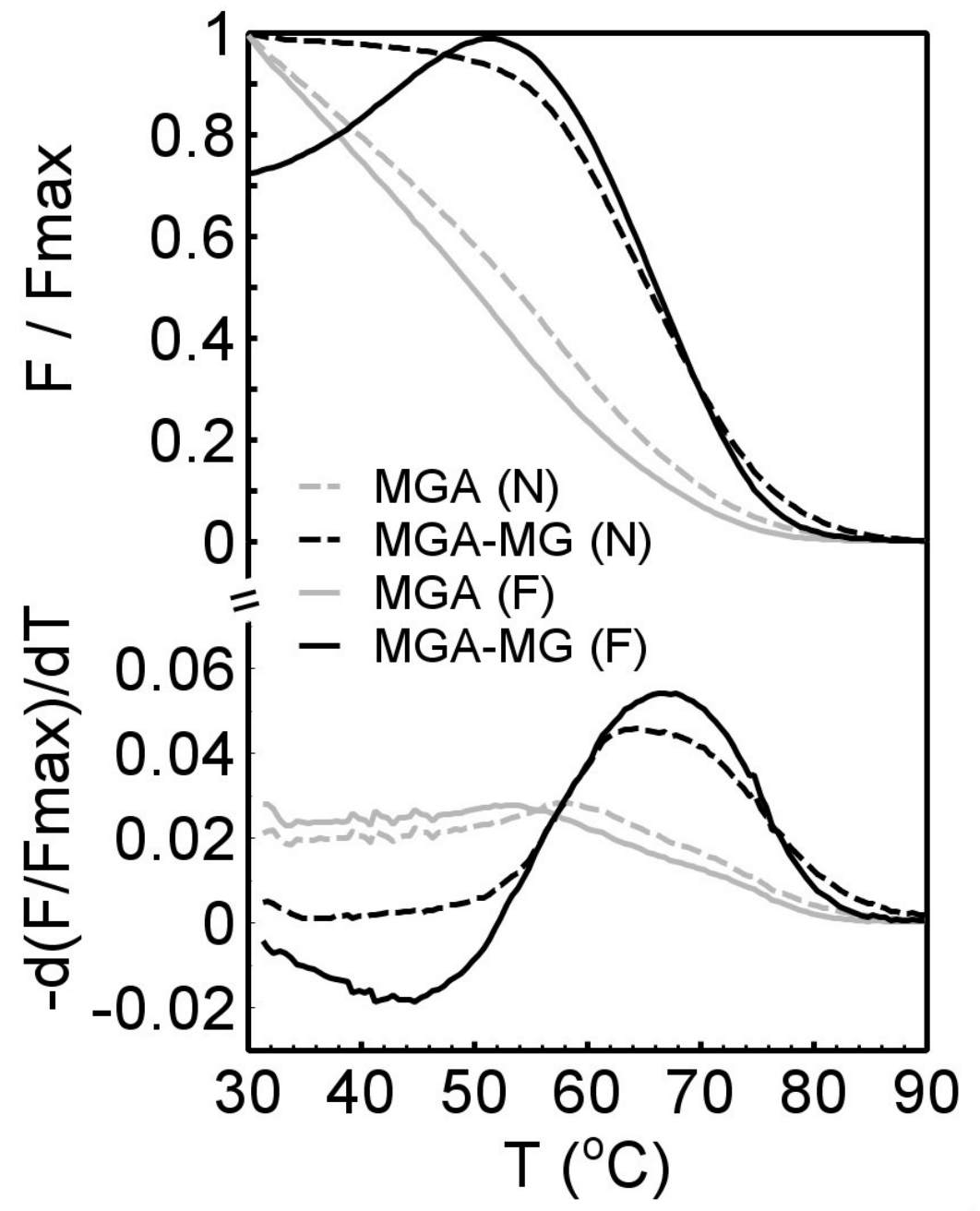


Fig. 5

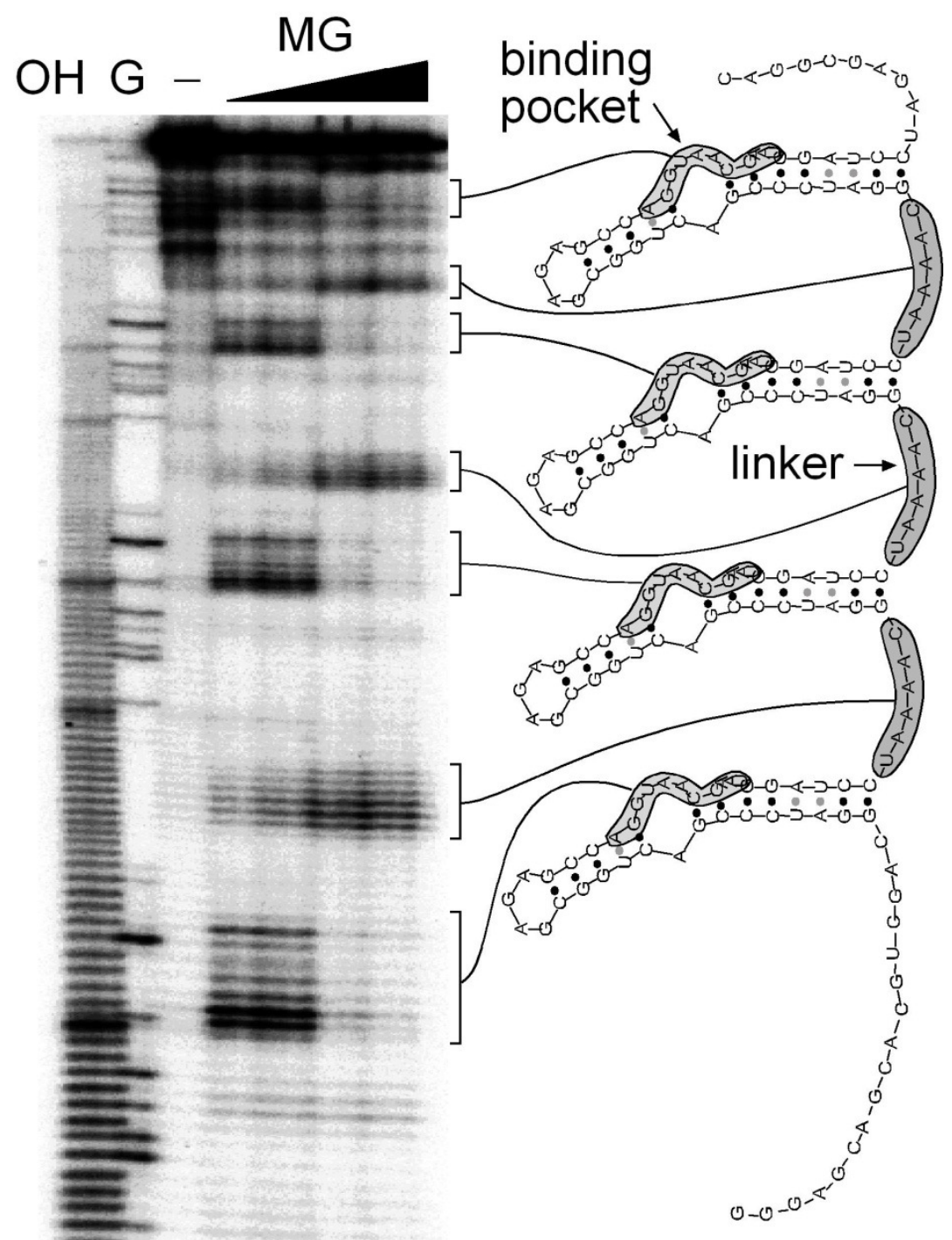


Fig. 6

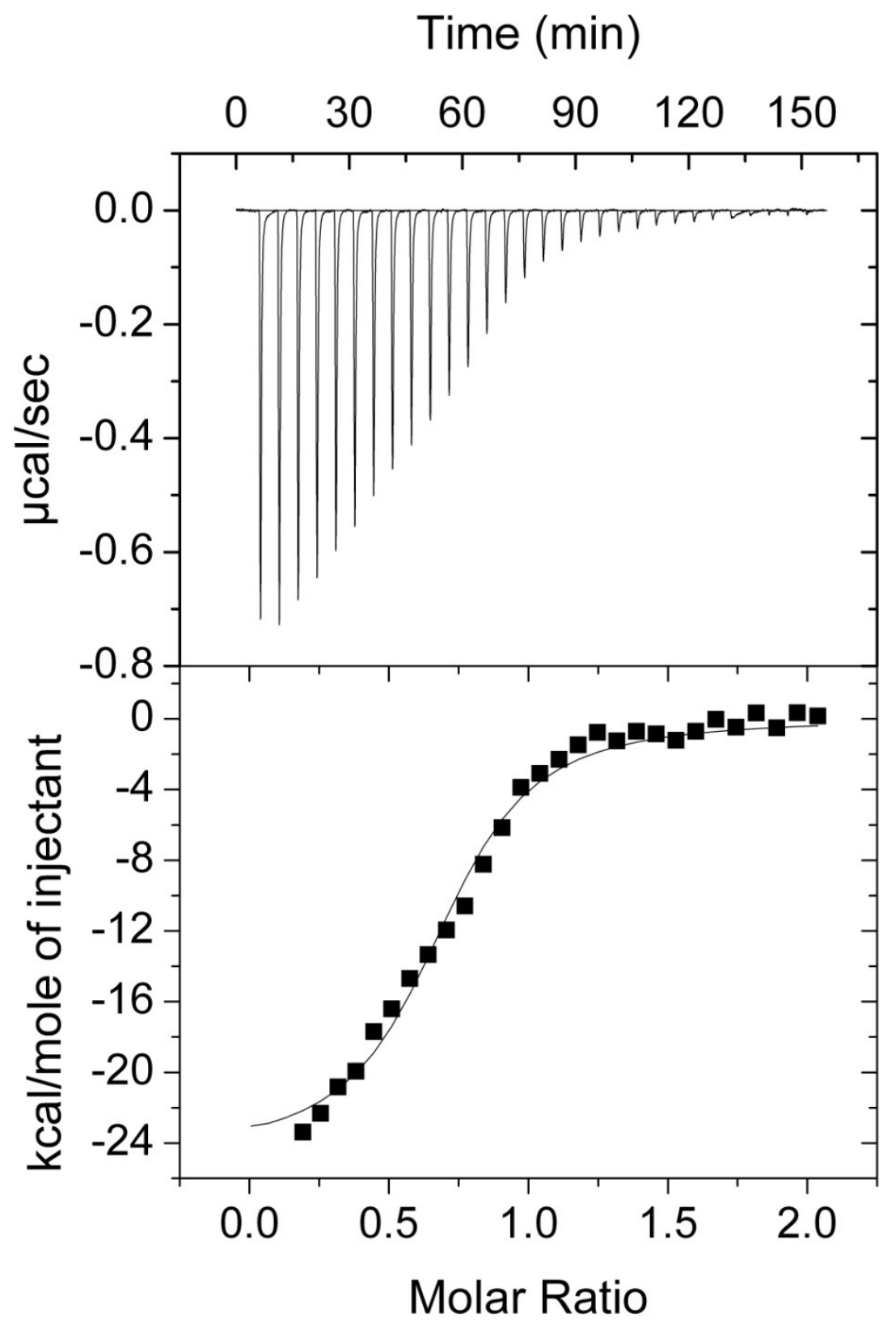


Fig. 7
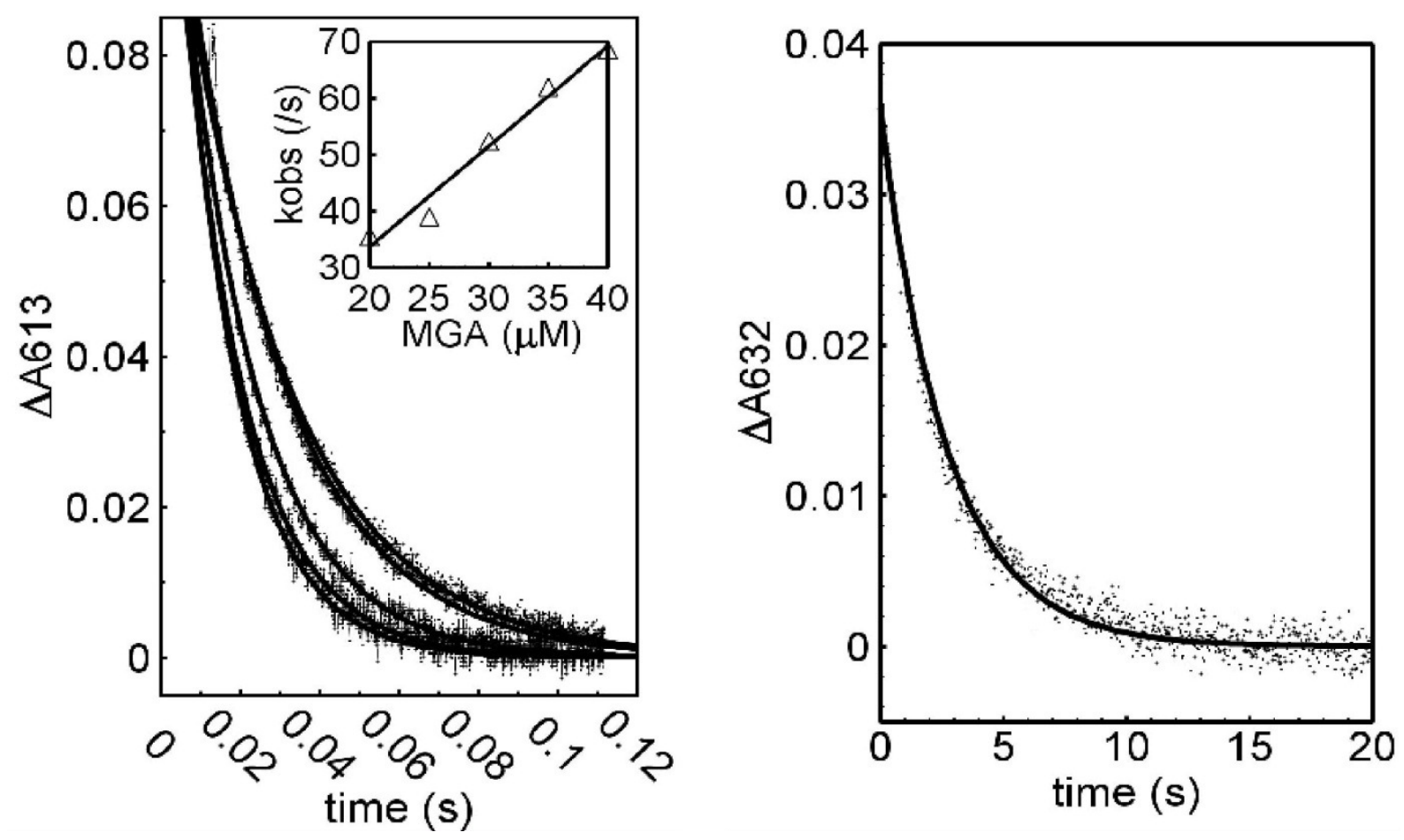
Fig. 8

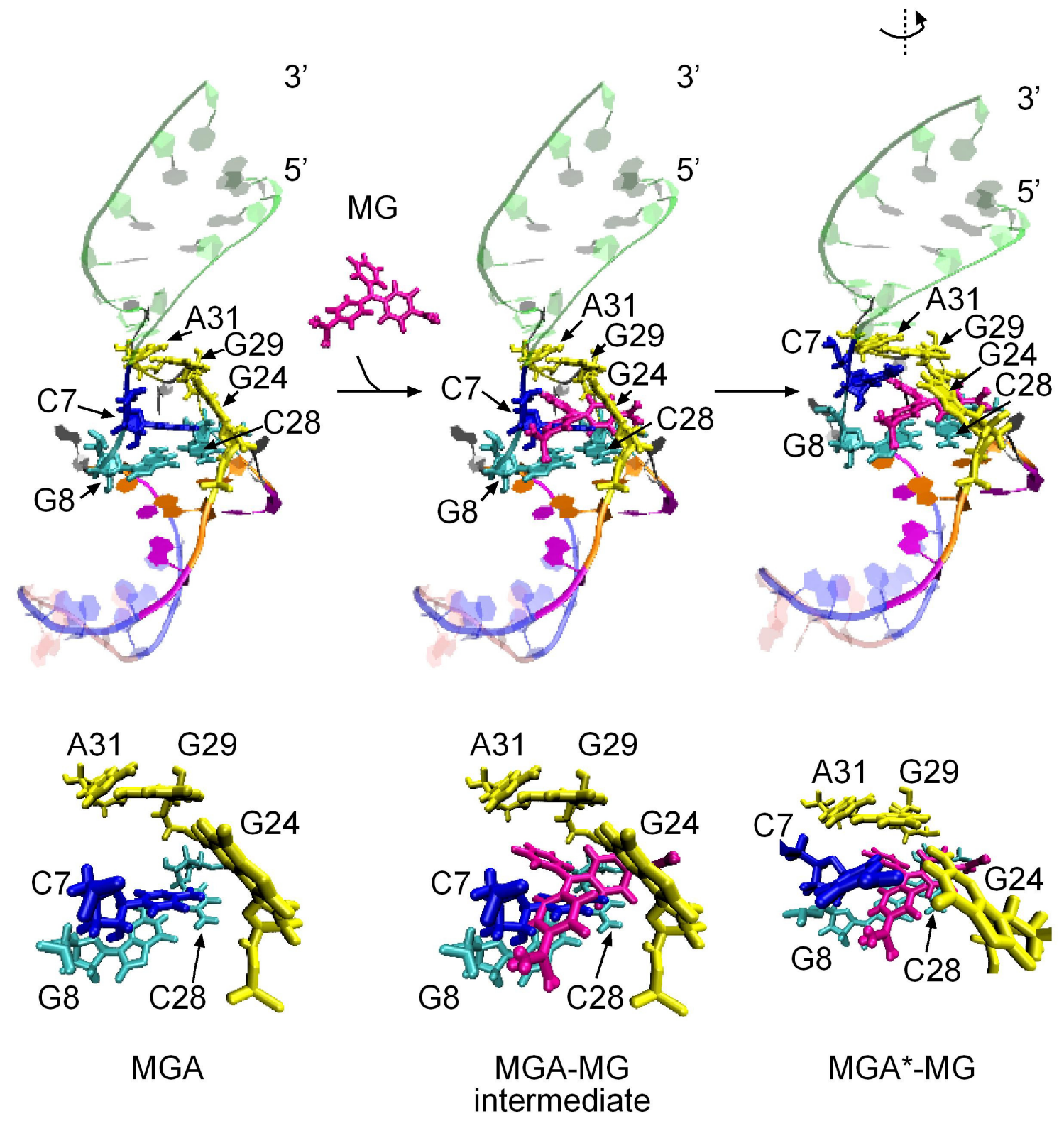




\section{CHAPTER 5. GENERAL CONCLUSIONS}

\section{CONTROLLING CHEMICAL REACTIVITY WITH APTAMERS}

A novel concept has been developed to control chemical reactivity by an aptamer-antisense oligonucleotide (AS) pair. The basic idea is to sequester a reactant inside the binding pocket of an aptamer that binds the reactant specifically and avidly. The sequestrated reactant is then unavailable to other reactants and the chemical reaction is thus inhibited. The sequestration of a reactant by an aptamer can be reversed by an AS against the aptamer. Through the aptamer-AS pair, a chemical reaction can be controlled in a reversible way. This concept has great potential in controlling metabolic reactions in vivo. For aptamers to sequester metabolites in vivo, they should have high affinity and specificity to compete with the enzymes for binding. We have demonstrated the feasibility of this concept using the MGA to control MG bleaching in vitro. This represents a first step to toward intervening in metabolism in vivo using the concept of the aptamer-AS pair. Future effort should be made to move this concept to work in vivo. Current methodologies, including gene knock-down, antisense oligonucleotide (AS) technology, small interfering RNA (siRNA), chemical inhibitors among others, mainly aim at controlling the activity or expression level of enzymes that catalyze metabolism by targeting genes or mRNA or protein of the enzymes. Aptamers are unique in their ability to target at the metabolite level. This ability gives our approach unique advantages in situations that a metabolic step is catalyzed by multiple enzymes or a single enzyme with gene redundancy. In this latter situation, knocking down a single gene or inhibiting a single enzyme will not inhibit the metabolic step. By targeting at the metabolite level, we can directly shut down a reaction reversibly with our aptamer-AS approach.

In addition, to control metabolism, aptamers could sequester endogenously toxic and reactive small molecules from damage to organisms. For example, an elevated level of methylglyoxal has been linked to neuronal and other diseases [1]. Developing aptamers against these small endogenously toxic compounds could be used for therapy. 
In conclusion, a novel concept has been developed to control chemical reactivity by aptamerAS pair, which has great potential in controlling metabolism and small toxic compounds among other applications.

\section{DYNAMICS OF AN APTAMER-TARGET INTERACTION REVEALED BY MD SIMULATION WITH EXPERIMENTAL VALIDATION}

MD simulation has been used to study the dynamics of nucleic acids in general and aptamertarget interactions in particular. The proper use of MD simulation yields results in good agreement with experimental data. The gap that we wanted to fill was to establish if MD simulation could extend structural and dynamical information beyond the limitations of current experimental techniques. Using the MGA-MG interaction as an example, we were able to discover the twisting force due to MG binding and used MD simulation to establish a model of the MG-MGA interaction as the combination of pre-organization and induced fit. The simulation results were validated by experimental studies. Thus, we integrated the MD simulations, thermodynamic and kinetic results to propose a two-step mechanism for MG-MGA interaction. Most importantly, we provided a simulated 3D-structure of unoccupied MGA with experimental validation. To my knowledge, the experimental 3D-structure of MGA has not been reported. In sum, we show that MD simulation can complement experimental approaches and can be used to dissect the mechanism of the MGA-MG interaction and provide a simulated 3D-structure of unoccupied MGA. With so many experimental structures of aptamer-target complex available, MD simulation could be used to explore the dynamics of many aptamer-target interactions. The dynamics of ions and water molecules in aptamer-target interaction

are also not easily studied by experimental approaches. MD simulation could provide a unique tool to decipher the role of ions and water and provide the basis of developing hypotheses for experimental validation [2].

Although we see MD simulation as a powerful tool to study aptamer-target interactions, we need to be cautious regarding its limitations. The standard force fields (CHARMM and AMBER) 
may not describe the behavior of divalent ions accurately and the simulation environment may not represent the real experimental conditions. Currently, typical MD simulations are run at $\sim 10 \mathrm{~ns}$ scale and many biological events happen at the $\sim \mu$ s and $\sim$ ms scale and therefore can not be revealed by MD simulations. To conclude, MD simulations, when performed in combination with experimental approaches, can be a powerful tool to study the dynamics of aptamer-target interactions that are not evident from the static 3D-structures. This approach can also provide new insights into the dynamics that is beyond the limitations of the conditions used for high-resolution data collection in NMR and crystallography..

\section{PERSPECTIVE}

Aptamer development has gone through 18 years. These studies have shown their application in medicine with the first aptamer drug, Macugen, approved by Food and Drug Administration at the end of 2004. Macugen is a pegylated VEGF aptamer that is used to treat age-related macular degeneration. SomaLogic and Archemix are two companies that own the proprietary rights to develop aptamers for diagnosis and therapy. In addition to their potential applications in medicine, aptamers also serve as good tools to study biological systems. Currently, aptamer selection is still done manually and it is time consuming. Although automation of selection has been attempted, no commercial instrument is available now. To meet the high demand of aptamers that recognize various targets, high throughput and highly efficient selection technology needs to be developed.

One unique feature of aptamers is their ability to distinguish the difference of even a single chemical group. Chemical modifications including acetylation, methylation, phosphorylation, ubiquitination, SUMOylation, ADP ribosylation, deimination and proline isomerization occur in proteins and some modifications also occur in nucleic acids. These modifications act as epigenetic codes and play an important role in biological systems. I see aptamer development to decipher the epigenetic code as an exciting field. 
In addition to aptamers selected in vitro, aptamers also exist in nature. Dr. Larry Gold first proposed nucleic acid-protein and nucleic acid-metabolite linkage maps in 1997. He also proposed a Genomic SELEX approach to study natural aptamers [3]. The hypothesis was confirmed by the discovery of riboswitches by Dr. Ronald Breaker's lab. Riboswtiches are RNA elements in the untranslated regions of mRNA that can specifically bind to metabolites and regulate transcription as well as translation. Riboswitches may be just the tip of the iceberg of natural aptamers. It has been proposed that genomes are filled with aptamers [4]. Appropriate approaches to select for these aptamers are in need of development. Discovering natural aptamers will be an exciting field in the near future.

\section{REFERENCES}

1. Gnerer, J. P., R. A. Kreber, and B. Ganetzky, wasted away, a Drosophila mutation in triosephosphate isomerase, causes paralysis, neurodegeneration, and early death. Proc Natl Acad Sci U S A, 2006. 103(41): p. 14987-93.

2. McDowell, S. E., N. Spackova, J. Sponer, and N. G. Walter, Molecular dynamics simulations of RNA: an in silico single molecule approach. Biopolymers, 2007. 85(2): p. 169-84.

3. Gold, L., D. Brown, Y. He, T. Shtatland, B. S. Singer, and Y. Wu, From oligonucleotide shapes to genomic SELEX: novel biological regulatory loops. Proc Natl Acad Sci U S A, 1997. 94(1): p. 59-64.

4. Gold, L., E. Brody, J. Heilig, and B. Singer, One, two, infinity: genomes filled with aptamers. Chem Biol, 2002. 9(12): p. 1259-64. 


\title{
APPENDIX. SELECTING APTAMERS AGAINST MOUSE LCN2 PROTEIN
}

\begin{abstract}
To develop aptamers as a tool to study mouse lipocalin 2 (Lcn2) function, Sequential Evolution of Ligands by Exponent enrichment (SELEX) was carried out. After 10 rounds of selection, 21 aptamer candidates were identified with 2 sequences constituting $50 \%$ of the candidate population. Secondary structure predictions showed that those candidates either adopt three way junction or multiple stem-loop structure. Further characterization of the aptamer is ongoing.
\end{abstract}

\section{INTRODUCTION}

Mature mouse Lcn 2 consists of 180 amino acids, not including the 20 amino acids of the signal peptide. Mouse Lcn2 was first discovered to be a growth factor induced and superinducible protein [1]. It has also been shown to be an acute phase protein [2]. Although many different functions have been proposed for mouse Lcn2, they are controversial and the in vivo role of this protein is unclear [3, 4]. Development of tools to study mouse Lcn2 will help to elucidate its function.

An aptamer is a ss-DNA or RNA that can bind to its target with high specificity and high affinity. Aptamers are selected in vitro by a procedure called SELEX [5, 6]. Compared with antibodies, aptamers are smaller in size. They can also be fine-tunable in vitro. Aptamers selected in vitro have already been shown to function in vivo to knockdown protein functions [713]. Here we proposed to select aptamers against mouse Lcn2 as a tool to study mouse Lcn2 function. 


\section{METHODS}

\section{Materials}

T7 RNA polymerase (recombinant protein prepared by Meiguan Yan), Inorganic pyrophosphatase (New England BioLabs, Boston, MA), NTPs and dNTPs (Fermentas, Glen Burnie, MD), and Taq DNA polymerase (GenScript, Piscataway, NJ), thermoscript reverse transcriptase (Invitrogen, Carlsbad, CA), DNase I (Epientre, Madison, WI), TOPO XL PCR cloning kit (Invitrogen, Carlsbad, CA ), Talon metal affinity resin (Clonetech, Mountain View, CA), $\alpha-{ }^{32}$ P-ATP (MP Biomedicals, Solon, OH), other chemicals (Fisher, Pittsburgh, PA). His-

mouse Lcn2 (recombinant protein prepared by Dr. Pierre Palo). Oligonucleotides 484, 485 and 487 were synthesized by Integrated DNA Technology (Coraville, IA) with standard desalting. Their sequences are:

Oligo 484: TAATACGACTCACTATAGGGAGACAAGAATAAACGCTCAA Oligo 485: GCCTGTTGTGAGCCTCCTGTCGAA

Oligo 487: GCCTGTTGTGAGCCTCCTGTCGAA(N53)TTGAGCGTTTATTCTTGTCTCCC SELEX procedure

Selection of aptamers against mouse Lcn2 was carried out using affinity chromatography as the separation methods [14]. First, ds-DNA were generated by Taq DNA polymerase extension with $600 \mu \mathrm{l}$ mixtures containing $1.67 \mu \mathrm{M}$ oligo 487, $3.33 \mu \mathrm{M}$ oligo 484, $0.5 \mathrm{mM}$ each dNTPs, 50 $\mathrm{mM} \mathrm{KCl}, 10 \mathrm{mM}$ Tris $\mathrm{HCl}\left(\mathrm{pH} 9.0\right.$ at $\left.25^{\circ} \mathrm{C}\right), 1.5 \mathrm{mM} \mathrm{MgCl}_{2}$, and $0.1 \%$ Triton $\mathrm{X}-100,0.05 \mathrm{U} / \mu \mathrm{l}$ Taq DNA polymerase. The reaction was carried out at $95^{\circ} \mathrm{C}$ for 3 min then $65^{\circ} \mathrm{C}$ for 5 min then $72^{\circ} \mathrm{C}$ for $40 \mathrm{~min}$. The extension products were gel purified and $0.357 \mathrm{nmol}$ of ds-DNA was used for in vitro transcription to generate the starting RNA pool for SELEX. For in vitro transcription, the reaction was done at $42^{\circ} \mathrm{C}$ for $1 \mathrm{~h}$, containing $5 \mathrm{mM}$ of each NTPs, $30 \mathrm{mM}$ Tris, $10 \mathrm{mM}$ DTT, $2 \mathrm{mM}$ spermidine, $20 \mathrm{mM}$ TritonX-100, $20 \mathrm{mM} \mathrm{MgCl}_{2}, \mathrm{pH} 8.5,0.7 \mu \mathrm{M}$ T7 RNA polymerase, 0.2 $\mathrm{U} / \mathrm{ml}$ inorganic pyrophosphatase and various amounts of DNAs (375 pmol DNA from the initial 
pool). The RNA was digested with DNase I and gel-purified. The purified RNA pool was incubated with his-mouse Lcn2 coupled to Talon Metal affinity resin in $50 \mathrm{mM} \mathrm{KH}_{2} \mathrm{PO}_{4}$, $150 \mathrm{mM} \mathrm{NaCl}, 5 \mathrm{mM} \mathrm{MgCl}_{2}, \mathrm{pH} 7.4$ at $23^{\circ} \mathrm{C}$ for $0.5-1 \mathrm{~h}$. The unbound RNA was then washed away with binding buffer and the bound RNA was eluted with $150 \mathrm{mM}$ imidazole, $\mathrm{pH}$ 7.2. The eluted RNA was then subjected to reverse transcription (RT)-PCR. In RT, $20 \mu 1$ reaction was carried out at $65^{\circ} \mathrm{C}$ for $1 \mathrm{~h}$ containing $1 \mathrm{mM}$ each dNTPs, $1.25-2.5 \mu \mathrm{M}$ oligo $485,0.75 \mathrm{U} / \mu \mathrm{l}$ thermoscript reverse transcriptase, 50mM Tris acetate $\left(\mathrm{pH} 8.4 @ 25^{\circ} \mathrm{C}\right), 75 \mathrm{mM}$ potassium acetate, $8 \mathrm{mM}$ magnesium acetate and various amounts of the captured RNA. One third of the RT product was subjected to PCR in $500 \mu \mathrm{l}$ reactions containing $1 \mathrm{mM}$ each dNTPs, $2 \mu \mathrm{M}$ oligo 484, $2 \mu \mathrm{M}$ oligo 485, $0.0125 \mathrm{U} / \mu \mathrm{l}$ Taq DNA polymerase, $50 \mathrm{mM} \mathrm{KCl,} 10 \mathrm{mM}$ Tris $\mathrm{HCl}(\mathrm{pH} 9.0$ at $25^{\circ} \mathrm{C}$ ), $1.5 \mathrm{mM} \mathrm{MgCl}_{2}$, and $0.1 \%$ Triton X-100. The PCR was carried out with 1 cycle of $93{ }^{\circ} \mathrm{C}$, $3 \mathrm{~min} ; 6-12$ cycles of $93{ }^{\circ} \mathrm{C}, 30 \mathrm{~s} ; 65^{\circ} \mathrm{C}, 1 \mathrm{~min} ; 72^{\circ} \mathrm{C}, 1 \mathrm{~min}$ and 1 cycle of $72{ }^{\circ} \mathrm{C}, 5 \mathrm{~min}$. The PCR product was then purified by phenol/chloroform extraction and ethanol precipitation. If necessary, $10 \mu \mathrm{Ci} \alpha-{ }^{32} \mathrm{P}$-ATP was added to the in vitro transcription reaction mixture to label the RNA. The radioactivity of the ${ }^{32} \mathrm{P}-\mathrm{RNA}$ bound to resin and that washed away was measured by liquid scintillation counter to monitor binding enrichment. The selection-amplification cycles were then repeated as specified in Table 1. In addition to the positive selection described above, negative selections were also carried out every 3 rounds using resin only incubated with RNA. Dilution of the mixture of RNA and mouse Lcn2 resin was used to increase selection stringency from round 3 to round 10 . At round 10, the RT-PCR products of the RNA binders were cloned using TOPO XL PCR cloning kit. A total of 72 clones were picked up for high throughput plasmid preparation and sequencing at Iowa state University DNA facility. The sequencing results were then analyzed with Vector NTI 10. 


\section{RESULTS}

\section{SELEX progress}

As shown in table 1, the total RNA binding to mouse $\mathrm{Lcn} 2$ increased from $0.4 \%$ at round 1 to $17.9 \%$ at round 10 while RNA binding to resin was $\sim 0.8 \%$. This indicated that the RNA pool had been enriched for mouse Lcn2 binders.

\section{Aptamers identified from SELEX}

A total of 72 clones from round 10 were subjected to sequencing, among which 56 meaningful sequences were obtained. Among the 56 sequences, there were 21 different molecules (figure 1). They were clustered into different families with A1 and A4 constituting greater than $50 \%$ of the population (figure 2). Secondary structure analysis by s-fold (http://sfold.wadsworth.org) $[15,16]$ showed that these aptamer candidates adopted either threeway junction or multiple stem-loop structure (figure 3).

\section{FUTURE WORK}

Further characterization of the aptamer candidates is under way. This includes determination of the Kd of the aptamers, minimization of the aptamers and studies of their effects on mouse Lcn2 function. 


\section{TABLES AND FIGURES}

Table 1. Summary of SELEX conditions for selecting mouse Lcn2 aptamers

\begin{tabular}{|c|c|c|c|c|c|c|c|c|}
\hline \multirow[t]{2}{*}{ round } & \multicolumn{3}{|c|}{ incubation } & \multirow{2}{*}{$\begin{array}{l}\text { Diluted } \\
\text { to }(\mu \mathrm{l})\end{array}$} & \multirow{2}{*}{$\begin{array}{l}\text { Wash } \\
(\mathrm{ml})\end{array}$} & \multirow{2}{*}{$\begin{array}{l}\text { Elute } \\
(\mathrm{ml})\end{array}$} & \multirow{2}{*}{$\begin{array}{l}\text { PCR } \\
\text { cycles }\end{array}$} & \multirow[t]{2}{*}{$\mathrm{B} \%$} \\
\hline & $\begin{array}{l}\operatorname{Lcn} 2 \\
(\mu \mathrm{M})\end{array}$ & $\begin{array}{l}\text { RNA } \\
(\mu \mathrm{M})\end{array}$ & $\begin{array}{l}\text { Volume } \\
(\mu \mathrm{l})\end{array}$ & & & & & \\
\hline 1 & 7.75 & 16 & 200 & & 5 & 0.4 & 10 & $0.4 \%$ \\
\hline 2 & 7.75 & 8.35 & 200 & & 5 & 0.4 & 10 & \\
\hline $3(-)$ & - & 148 & 30 & & 0.2 & & & \\
\hline $3(+)$ & 50 & 148 & 30 & 400 & 5 & 0.4 & 10 & \\
\hline 4 & 50 & 74.7 & 30 & 200 & 5 & 0.4 & 8 & \\
\hline 5 & 43 & 31 & 30 & 400 & 5 & 0.4 & 9 & \\
\hline $6(-)$ & - & 47 & 30 & & 0.05 & & & \\
\hline $6(+)$ & 19 & 21 & 65 & 200 & 5 & 0.4 & 7 & $1.8 \%$ \\
\hline 7 & 38.75 & 50 & 20 & 200 & 5 & 0.4 & 8 & \\
\hline 8 & 38.75 & 50 & 20 & 200 & 5 & 0.4 & 6 & \\
\hline $9(-)$ & - & 40 & 50 & & 0.25 & & & $0.8 \%$ \\
\hline $9(+)$ & 38.75 & 40 & 10 & 200 & 5 & 0.4 & 11 & $9.8 \%$ \\
\hline 10 & 46.5 & 63.5 & 10 & 500 & 10 & 0.4 & 10 & $17.9 \%$ \\
\hline
\end{tabular}


Figure 1. 21 different species obtained from 56 clones of utc-SELEX
(1) 1
10
20
30
50
60
70
80
90 107
A1 (1) GGGAGACAAGAAUAAACGCUCAAC--CUCCACAGGCACGCUUUGUUGACCUCCGGCUCAUACCUUUUUCGAAGACAA-GCUUCGACAGGAGGCUCACAA----CAGGC E3 (1) GGGAGACAAGAAUAAAACGCUCAAU--CUCCACCGGCACGCUUUGUUGACCUCCGGCUCAUACCUUUUCGAAGACAA-GCUUCGACAGGAGGCUCACAA-----CAGGC
A3 (1) GGGAGACAAGAAUAAACGCUCAACC-CUGAGGC-UCUGCUGUGC-UCAUGCCACUCUCCCUUCCUUUUCGUCUAGUGCCGUUCGACAGGAGGCUCACAA----CAGGC
H1 (1) GGGAGACAAGAAUAAAACGCUCAAGCUCUGGUGCCUGUGCUUCGCGUUAUUUCCCUCUCGCUU--UUUGGGGU-GUUCCUUUCGACAGGAGGCUCACAA----CAGGC
C1 (1) GGGAGACAAGAAUAAACGCUCAACU--UGUGCCUGCUGAUCGGUCUGUUUUCUUUCUGCCGCUUUUUGCGCCU-GUUCUCUUCGACAGGAGGCUCACAA----CAGGC
C2 (1) GGGAGACAAGAAUAAACGCUCAAGUGAUGUGCCUGCGCUUGAUCCUUCUUUACC-CGUCCUGCUGCCUGUUU--CGUUCUUCGACAGGAGGCUCACAA----CAGGC
C9 (1) GGGAGACAAGAAUAAACGCUCAACUGUGCCUGUUCGGGGUUAUUCUUGUUUAGC-CUCACUUGUGUGGGACU--CUAUGUUCGACAGGAGGCUCACAA----CAGGC
A4 (1) GGGAGACAAGAAUAAACGCUCAA-GUCCCAUUGGUCGUCCUCUUCACUGUUGCGGCCUCGGGGACUGUUUCACUC-UGUUUU-CGACAGGAGGCUCACAA----CAGGC
H9 (1) GGGAGACAAGAAUAAAACGCUCAA-GUCCCAUUGUCGUCCUCUUCACUGUUGCGGCCUCGGGGACUGUUCACUC-UGUUUUUCGACAGGAGGCUCACAA----CAGGC
A6 (1) GGGAGACAAGAAUAAACGCUCAAGGGCCCUAUGUUGUGGGUUGGAUUUAGUAUCGAUGGC----CAUUCACUUCUGGAGUUCGACAGGAGGCUCACAA----CAGGC
A9 (1) GGGAGACAAGAAUAAAACGCUCAA-GUGCCUGUGCUUUUGCCUUUCUCCCUUUCUCUUGGCUGGUUCCUGCUCC--CCGGUUCGACAGGAGGCUCACAA----CAGGC
B3 (1) GGGAGACAAGAAUAAACGCUCAA-GUGCCUGUAGUGCAUCUGCUCCUUCCUGCAUGUGGCU--UUCCUGCCCUUUCUUUUUCGACAGGAGGCUCACAA----CAGGC
B9 (1) GGGAGACAAGAAUAAACGCUCAAUGUGCCCGUUGCUGCCUGUCUCUGCCUCUUUCGUG-CUCGUUACU-UGUGCCUGGUUUCGACAGGAGGCUCACAA----CAGGC
G1 (1) GGGAGACAAGAAUAAAACGCUCAACGUGCCUGUGGC--UCUUUCUACGCCCCGC-CUUG-CUUUGUUUCUGUUCCUCGGGUUUCGACAGGAGGCUCACAA-----CAGGC
D7 (1) GGGAGACAAGAAUAAAACGCUCAACGUGCCUGUAUCG-UCUUGCCCUGUCGCCC-CCUG-UAGUUUGCUCUUUGGUCUGUCUUCGACAGGAGGCUCACAA----CAGGC
B7 (1) GGGAGACAAGAAUAAACGCUCAACGUCCGUUUACUCGACCCUCUGUGAUGAGCC-CUCUCUCGUUGUUGUGC---CUGGUUCGACAGGAGGCUCACAA----CAGGC
C3 (1) GGGAGACAAGAAUAAACGCUCAA-GUCUGUUGAUGCUGCUGCCACUGAAUACUUGGCACC--GUGCCUAUGCCAUCGAAUUCGACAGGAGGCUCACAA----CAGGC
B1 (1) GGGCGA----AUUGGGCCCUCUA-----GAUGCAUGCUCGAGCGGCCGCCAGUGUGAUUGGAUAUCUGCUGUUU-----GCCUUCGACAGGAGGCUCACAA-----CAGGC
B2 (1) GGGCGA-ÄUUCUGCAGAUAUCCAUCACACUGGCGGCCGCUCGAGCAUGCAUCUAGAGGGCCCAAUUUCGCCCU------AU-AGUGAGUCGUAUUACAAUUCACUGGC 
Figure 2. Phylogenetic tree of Lcn2-SELEX according to alignment in Vector NTI

\begin{tabular}{|cc}
\hline$A 1(0.0085)$ & $14 / 56$ \\
E3 (0.0115) & $2 / 56$ \\
C5 (0.1146) & $2 / 56$ \\
\hline A3 (0.0825) & $2 / 56$ \\
H1 (0.0824) & $1 / 56$ \\
C1 (0.1222) & $2 / 56$ \\
C2 (0.1423) & $1 / 56$ \\
C9 (0.1477) & $2 / 56$ \\
A4 (0.0017) & $12 / 56$ \\
H9 (-0.0017) & $2 / 56$ \\
A6 (0.1403) & $3 / 56$ \\
\hline A9 (0.1096) & $2 / 56$ \\
B3 (0.1148) & $1 / 56$ \\
B9 (0.1049) & $1 / 56$ \\
G1 (0.0992) & $2 / 56$ \\
D7 (0.1051) & $2 / 56$ \\
B7 (0.1331) & $1 / 56$ \\
C3 (0.1482) & $1 / 56$ \\
\hline B1 (0.1941) & $2 / 56$ \\
\hline
\end{tabular}


Figure 3. Representative secondary structures of mouse Lcn2 aptamer candidates

A1

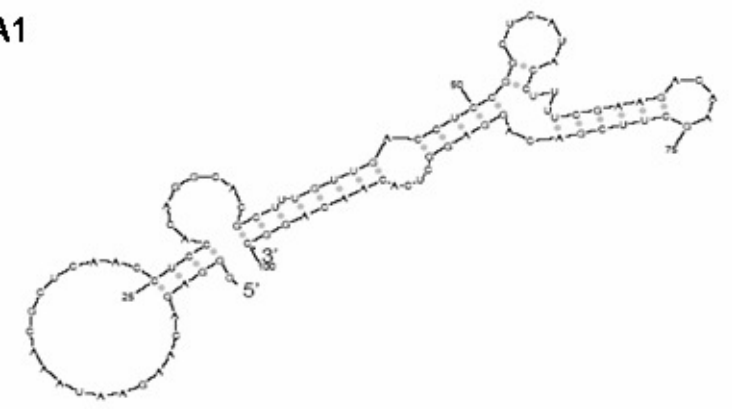

C5

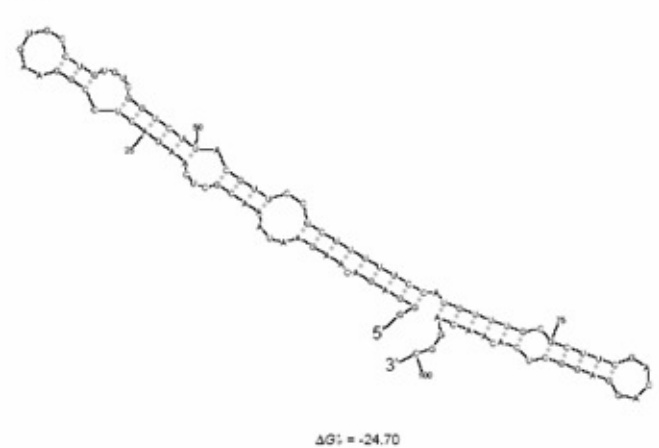

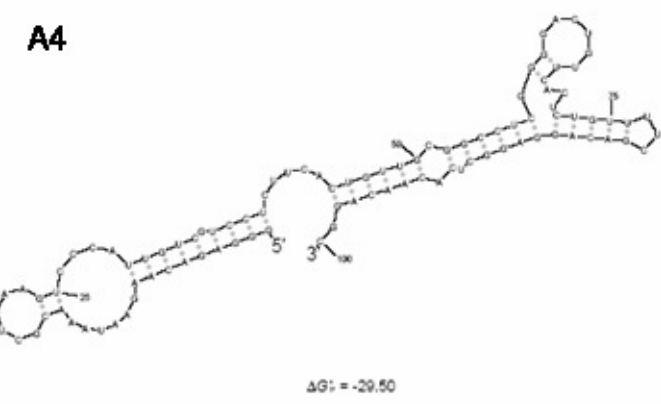

A6

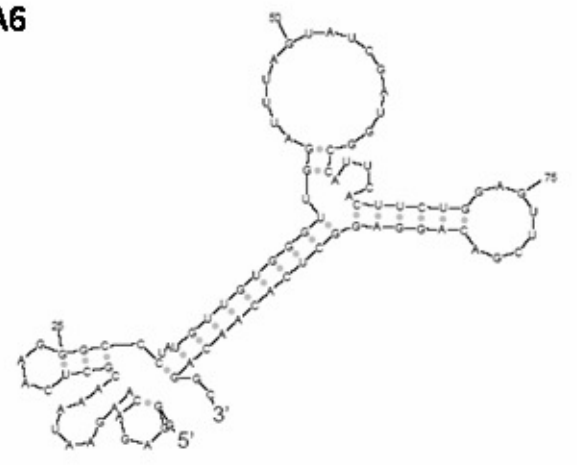

so: $=\cdot 20.70$ 


\section{REFERENCES}

1. Nilsen-Hamilton, M., R. T. Hamilton, and G. A. Adams, Rapid selective stimulation by growth factors of the incorporation by $B A L B / C 3 T 3$ cells of [35S]methionine into a glycoprotein and five superinducible proteins. Biochem Biophys Res Commun, 1982. 108(1): p. 158-66.

2. Liu, Q., J. Ryon, and M. Nilsen-Hamilton, Mouse Lcn2: a mouse acute phase protein expressed in the uterus around birth. Mol Reprod Dev, 1997. 46(4): p. 507-14.

3. Grzyb, J., D. Latowski, and K. Strzalka, Lipocalins - a family portrait. J Plant Physiol, 2006. 163(9): p. 895-915.

4. Flower, D. R., The lipocalin protein family: structure and function. Biochem J, 1996. 318 ( Pt 1): p. 1-14.

5. Ellington, A. D. and J. W. Szostak, In vitro selection of RNA molecules that bind specific ligands. Nature, 1990. 346(6287): p. 818-22.

6. Tuerk, C. and L. Gold, Systematic evolution of ligands by exponential enrichment: RNA ligands to bacteriophage T4 DNA polymerase. Science, 1990. 249(4968): p. 505-10.

7. Bai, J., N. Banda, N. S. Lee, J. Rossi, and R. Akkina, RNA-based anti-HIV-1 gene therapeutic constructs in SCID-hu mouse model. Mol Ther, 2002. 6(6): p. 770-82.

8. Chaloin, L., M. J. Lehmann, G. Sczakiel, and T. Restle, Endogenous expression of a high-affinity pseudoknot RNA aptamer suppresses replication of HIV-1. Nucleic Acids Res, 2002. 30(18): p. 4001-8.

9. Lee, H. K., Y. S. Choi, Y. A. Park, and S. Jeong, Modulation of oncogenic transcription and alternative splicing by beta-catenin and an RNA aptamer in colon cancer cells. Cancer Res, 2006. 66(21): p. 10560-6.

10. Mi, J., X. Zhang, Z. N. Rabbani, Y. Liu, S. K. Reddy, Z. Su, F. K. Salahuddin, K. Viles, P. H. Giangrande, M. W. Dewhirst, B. A. Sullenger, C. D. Kontos, and B. M. Clary, RNA aptamer-targeted inhibition of NF-kappaB suppresses non-small cell lung cancer resistance to doxorubicin. Mol Ther, 2008. 16(1): p. 66-73.

11. Nishikawa, F., N. Kakiuchi, K. Funaji, K. Fukuda, S. Sekiya, and S. Nishikawa, Inhibition of HCV NS3 protease by RNA aptamers in cells. Nucleic Acids Res, 2003. 31(7): p. 1935-43.

12. Sarraf-Yazdi, S., J. Mi, B. J. Moeller, X. Niu, R. R. White, C. D. Kontos, B. A. Sullenger, M. W. Dewhirst, and B. M. Clary, Inhibition of in vivo tumor angiogenesis and growth via systemic delivery of an angiopoietin 2-specific RNA aptamer. J Surg Res, 2008. 146(1): p. 16-23.

13. Shi, H., B. E. Hoffman, and J. T. Lis, RNA aptamers as effective protein antagonists in a multicellular organism. Proc Natl Acad Sci U S A, 1999. 96(18): p. 10033-8.

14. Fitzwater, T. and B. Polisky, A SELEX primer. Methods Enzymol, 1996. 267: p. 275-301.

15. Ding, Y. and C. E. Lawrence, A statistical sampling algorithm for RNA secondary structure prediction. Nucleic Acids Res, 2003. 31(24): p. 7280-301.

16. Ding, Y., C. Y. Chan, and C. E. Lawrence, RNA secondary structure prediction by centroids in a Boltzmann weighted ensemble. Rna, 2005. 11(8): p. 1157-66. 


\section{ACKNOWLEDGEMENTS}

There are many persons to thank for during my $\mathrm{Ph} . \mathrm{D}$. study.

I thank my major professor Dr. Marit Nilsen-Hamilton. Marit has been a great advisor for me. Her wisdom and diligence inspires me in research. Sometimes the research doesn't go smoothly but Marit has been very supportive and patient with me and she guides me through all those difficulties. I am grateful to have her as my advisor.

I thank my previous POS committee members, Dr. Amy Andreotti, Dr. Janice Buss and Dr. Gloria Culver for their guidance.

I thank my current POS committee member Dr. Drena Dobbs, Dr. Monica Lamm, Dr. Gustavo MacIntosh and Dr. Allen Miller for their guidance and their advice on the research projects.

I thank Dr. Mark Hargrove for his advice on cavity assay and his support for stopped-flow kinetics study. I also thank Dr. Gaya Amarasinghe for his advice on melting curve measurement and his help on NMR data interpretation.

I thank Dr. Kamel Harrata, Dr. Shu Xu and Dr. Bruce Fulton for carrying out Mass spectrometry and NMR spectrometry for characterization of MG bleaching and MGA-MG spectra.

I also thank my lab members. I thank Lee Bendickson for his technical support and help and providing p4MG plasmid. I thank Dr. Pierre Palo for providing uterocalin protein. I thank a group of persons involving in SELEX methodology development in our lab including Dr. Richard Hamilton, Dr. Xiangyu Cong, Samir Mehanovic, Allison Pappas, Ying Liu and Marjan Mokhtarian. I thank Wei Zhao for helping me with CoPlot and uterocalin background information and sharing with me some writing tips. I also thank other lab members for their technical support and discussions.

Finally I am indebted to my family. I thank my parents for supporting me to study abroad. I thank my wife Meiguan Yan and my son Xiangyuan Andrew Wang for their understanding and support. Especially, I thank my sister, Qingdie Wang for her long-term support and her taking on my responsibility to take care of my parents back in China during my Ph.D. study in U.S.A. 